Consortium for Advanced

Simulation of LWRs

\title{
CTF Theory Manual
}

R. Salko ${ }^{1}$, M. Avramova ${ }^{2}$, A. Wysocki ${ }^{1}$, A. Toptan ${ }^{2}$, J.

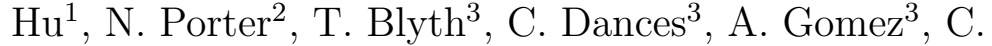
Jernigan $^{4}$, and J. Kelly ${ }^{5}$

${ }^{1}$ Oak Ridge National Laboratory

${ }^{2}$ North Carolina State University

${ }^{3}$ The Pennsylvania State University

${ }^{4}$ Holtec International

${ }^{5}$ US Nuclear Regulatory Commission

$3 / 24 / 2019$

FisVER

VERA QA Document - This document was prepared under the ORNL VERA Quality Assurance Program in accordance with procedure VERA-QA-003. The OFFICIAL COPY of this document is the electronic version in the VERA Documents Repository. Before using a printed copy, verify that it is the most current version by checking the Revision ID against the electronic version.

Approved for Public Release
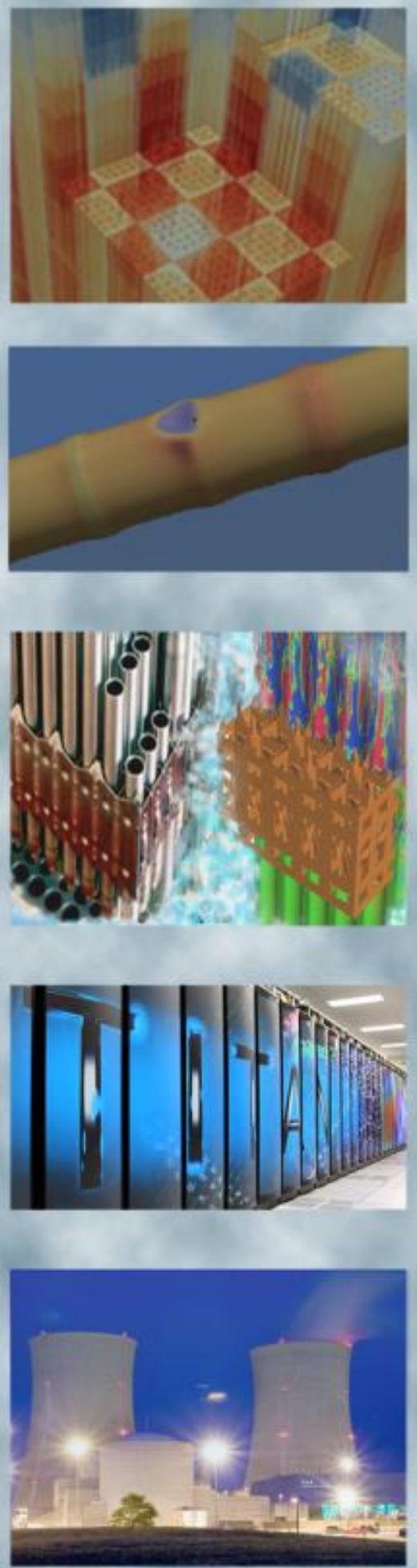


\section{Revision Log}

\begin{tabular}{rrrr}
\hline Revision & Date & Affected Pages & Revision Description \\
\hline 0 & $3 / 24 / 2019$ & All & Initial Release \\
& & & \\
& & & \\
\hline
\end{tabular}

\section{Document pages that are:}

$\begin{array}{ll}\text { Export Controlled: } & \text { None } \\ \text { IP/Proprietary/NDA Controlled: } & \text { None } \\ \text { Sensitive Controlled: } & \text { None } \\ \text { Unlimited: } & \text { All }\end{array}$

This document was prepared as an account of work sponsored by an agency of the United States Government. Neither the United States Government nor any agency thereof, nor any of their employees, makes any warranty, express or implied, or assumes any legal liability or responsibility for the accuracy, completeness, or usefulness of any information, apparatus, product, or process disclosed, or represents that its use would not infringe privately owned rights. Reference herein to any specific commercial product, process, or service by trade name, trademark, manufacturer, or otherwise, does not necessarily constitute or imply its endorsement, recommendation, or favoring by the United States Government or any agency thereof. The views and opinions of authors expressed herein do not necessarily state or reflect those of the United States Government or any agency thereof. 


\section{The North Carolina State University}

Department of Nuclear Engineering

Reactor Dynamics and Fuel Modeling Group

\section{NC STATE UNIVERSITY}

\section{CTF Theory Manual}

July 29, 2019 


\section{CTF Theory Manual}

\section{Approvals:}

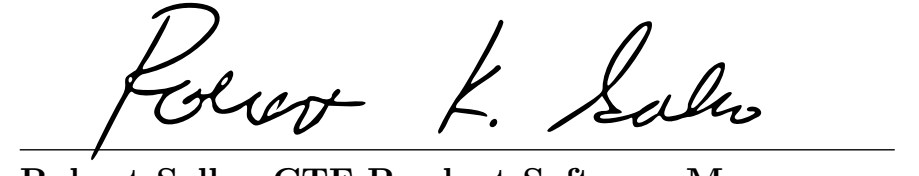

Robert Salko, CTF Product Software Manager

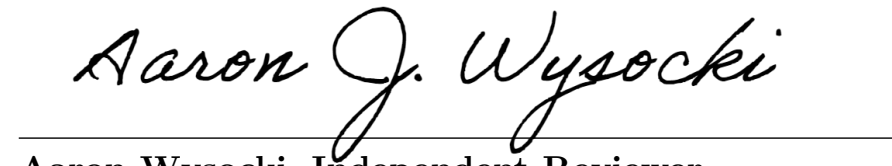

Aaron Wysocki, Independent Reviewer

\section{$7 / 29 / 2019$}

Date

Date 


\section{Executive Summary}

Coolant-Boiling in Rod Arrays-Two Fluids (COBRA-TF) is a thermal/hydraulic (T/H) simulation code designed for light water reactor (LWR) vessel analysis. It uses a two-fluid, three-field (i.e. fluid film, fluid drops, and vapor) modeling approach. Both sub-channel and 3D Cartesian forms of 9 conservation equations are available for LWR modeling. The code was originally developed by Pacific Northwest Laboratory in 1980 and had been used and modified by several institutions over the last few decades.

COBRA-TF also found use at the Pennsylvania State University (PSU) by the Reactor Dynamics and Fuel Modeling Group (RDFMG) and has been improved, updated, and subsequently re-branded as CTF. As part of the improvement process, it was necessary to generate sufficient documentation for the open-source code which had lacked such material upon being adopted by RDFMG. This document serves mainly as a theory manual for CTF, detailing the many two-phase heat transfer, drag, and important accident scenario models contained in the code as well as the numerical solution process utilized. Coding of the models is also discussed, all with consideration for updates that have been made when transitioning from COBRA-TF to CTF. Further documentation outside of this manual is also available at RDFMG which focus on code input deck generation and source code global variable and module listings. 


\section{Contents}

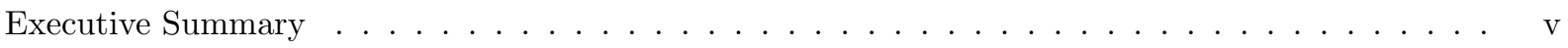

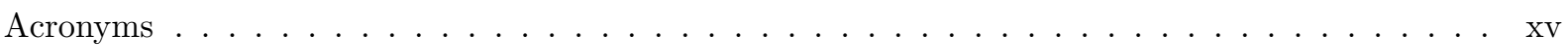

1 Introduction 1

2 Conservation Equations and Numerical Solution $\quad 3$

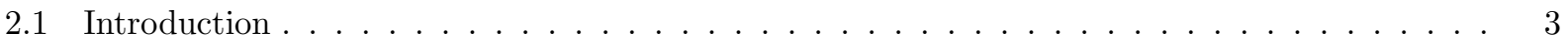

2.2 Conservation Equations $\ldots \ldots \ldots \ldots \ldots \ldots \ldots \ldots \ldots \ldots \ldots$

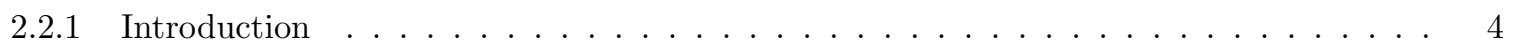

$2.2 .2 \quad$ Generalized Conservation Equations . . . . . . . . . . . . . . . . . . . 4

2.2.2.1 Generalized Phasic Mass Conservation Equation . . . . . . . . . . . . . . . 4

2.2.2.2 Generalized Phasic Momentum Conservation Equation . . . . . . . . . . . 5

2.2.2.3 Generalized Phasic Energy Conservation Equation . . . . . . . . . . . . . 7

2.2.3 CTF Expanded Conservation Equations in Cartesian Form . . . . . . . . . . . . 7

2.2.3.1 Mass Continuity Conservation Equation in Cartesian Coordinates . . . . . . 8

2.2.3.2 Momentum Conservation Equation in Cartesian Coordinates . . . . . . . . 8

2.2.3.3 Energy Equation in Cartesian Coordinates . . . . . . . . . . . . . . . . . 10

2.2.4 CTF Conservation Equations in Sub-Channel Form . . . . . . . . . . . . . . . 10

2.2.4.1 Mass Conservation Equations in Sub-Channel Coordinates . . . . . . . . . 11

2.2.4.2 Momentum Conservation Equations in Sub-Channel Coordinates . . . . . . . 11 
2.2.4.3 Energy Conservation Equations in Sub-Channel Coordinates . . . . . . . . . 12

2.3 Discretized Conservation Equations and Computational Mesh . . . . . . . . . . . . . 13

2.3.1 CTF Flow Volume Meshing Approach . . . . . . . . . . . . . . . . . . 13

2.3.2 Finite-Difference Conservation Equations . . . . . . . . . . . . . . . . . 16

2.3.2.1 Finite-Difference Mass Conservation Equation . . . . . . . . . . . . . . 16

2.3.2.2 Finite-Differenced Momentum Conservation Equation . . . . . . . . . . . . 18

2.3.2.3 Finite-Differenced Energy Conservation Equations . . . . . . . . . . . . . . 30

2.4 Numerical Solution . . . . . . . . . . . . . . . . . . . . . . . . . . 31

2.4.1 Linearization and Solution of the Momentum Equations . . . . . . . . . . . . . . . 32

2.4.2 Linearization and Solution of Mass and Energy Equations . . . . . . . . . . . . . . 35

2.4.3 Solution of the Pressure Matrix . . . . . . . . . . . . . . . . . . . 38

2.4.4 Back-substitution and Velocity Correction . . . . . . . . . . . . . . . 39

2.4.5 Convergence Criteria . . . . . . . . . . . . . . . . . . . . . . . 40

2.4.5.1 Time step Size Control . . . . . . . . . . . . . . . . . . . . 40

2.4.5.2 Numerical Stability ......................... 41

2.4.5.3 Pseudo-steady-state Option . . . . . . . . . . . . . . . . . . . 42

2.5 Boron Tracking and Precipitation Modeling . . . . . . . . . . . . . . . . . . . . 44

2.5.1 Boron Tracking Model . . . . . . . . . . . . . . . . . . . . . . 44

2.5.1.1 First Order Accurate Upward Difference Scheme . . . . . . . . . . . . . . . 45

2.5.1.2 Second Order Godunov Scheme . . . . . . . . . . . . . . . . . . 47

2.5.2 Boron Precipitation Model . . . . . . . . . . . . . . . . . . . 50

3 Flow Regime Map 53

3.1 Introduction . . . . . . . . . . . . . . . . . . . . . . . 53

3.2 Normal Wall Flow Regimes . . . . . . . . . . . . . . . . . . . . . 53

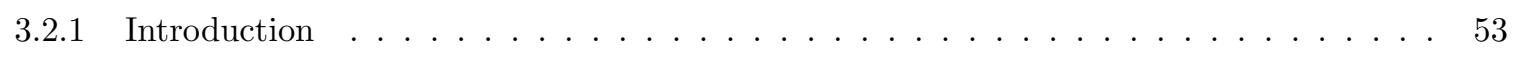

3.2.2 Small Bubble Regime . . . . . . . . . . . . . . . . . . . . . . . 58 
3.2.3 Small-to-Large Bubble Regime . . . . . . . . . . . . . . . . . . . . . . 59

3.2.4 Churn/Turbulent Flow Regime . . . . . . . . . . . . . . . . . . . . 62

3.2.5 Annular/Mist Flow Regime .......................... 63

3.3 Hot Wall Flow Regimes . . . . . . . . . . . . . . . . . . . . . . . . . 64

3.3.1 Introduction ............................. 64

3.3.2 Inverted Annular Flow Regime . . . . . . . . . . . . . . . . . . . . . . . . . . 64

3.3.3 Dispersed Droplet Flow Regime . . . . . . . . . . . . . . . . . . . 67

3.3.4 Falling Film Regime . . . . . . . . . . . . . . . . . . . . . . 67

3.3.5 Top Deluge Flow Regime ............................ 67

3.4 Interfacial Area Transport Equation . . . . . . . . . . . . . . . . . . . . . . 68

4 Macro-Mesh Cell Closure Models 70

4.1 Introduction . . . . . . . . . . . . . . . . . . . . . 70

4.2 Wall Shear and Form Loss Models . . . . . . . . . . . . . . . . . . . . 70

4.2 .1 Friction Loss Model . . . . . . . . . . . . . . . . . . . . . . . 71

4.2 .2 Form Loss Model . . . . . . . . . . . . . . . . . . . . . . . . . . 73

4.2.3 Simplification of Wall/Form Loss Model . . . . . . . . . . . . . . . . . . . . 73

4.3 Inter-cell Drag Models . . . . . . . . . . . . . . . . . . . . . . . 76

4.4 Turbulent Mixing and Void Drift . . . . . . . . . . . . . . . . . . . . . 79

4.4.1 Turbulent mixing . . . . . . . . . . . . . . . . . . . . . . . . 80

4.4 Void Drift . . . . . . . . . . . . . . . . . . . . . . . 82

4.4.3 Mixing Coefficient ........................... . . 84

5 Micro-Mesh Cell Closure Models 88

5.1 Interfacial Drag Models . . . . . . . . . . . . . . . . . . . . . . 88

5.1.1 Small Bubble Regime ........................... . . 89

5.1 .2 Slug Regime ... . . . . . . . . . . . . . . . . . . . 92

Consortium for Advanced Simulation of LWRs niii $\quad$ CASL-U-2018-1715-000 
5.1 .3 Dispersed Droplet Flow Regime . . . . . . . . . . . . . . . . . . . . . . 93

5.1 .4 Annular/Mist Regime . . . . . . . . . . . . . . . . . . . . . . . 94

5.1 .5 Churn/Turbulent Flow Regime . . . . . . . . . . . . . . . . . . . . . 95

5.1 .6 Inverted Annular Flow Regime . . . . . . . . . . . . . . . . . . . 96

5.1 .7 Falling Film Regime $\ldots \ldots \ldots \ldots \ldots$

5.1 .8 Top Deluge Flow Regime . . . . . . . . . . . . . . . . . . . . . . . 97

5.2 Interfacial Heat Transfer Models . . . . . . . . . . . . . . . . . . . . . . 98

$5.2 .1 \quad$ Small Bubble Flow Regime . . . . . . . . . . . . . . . . . . . . . . . . . 99

5.2 .2 Slug Regime . . . . . . . . . . . . . . . . . . . . . . 101

5.2 .3 Dispersed Droplet Flow Regime . . . . . . . . . . . . . . . . . . . . . . . . . . 102

5.2 .4 Annular $/$ Mist Flow Regime . . . . . . . . . . . . . . . . . . . . . 103

5.2 .5 Churn-Turbulent Flow Regime . . . . . . . . . . . . . . . . . . . . . 104

5.2 .6 Falling Film and Deludge Flow Regimes . . . . . . . . . . . . . . . . 105

5.2 .7 Inverted Annular Flow Regime . . . . . . . . . . . . . . . . . . 106

5.3 Entrainment and De-Entrainment Models . . . . . . . . . . . . . . . . . . . 106

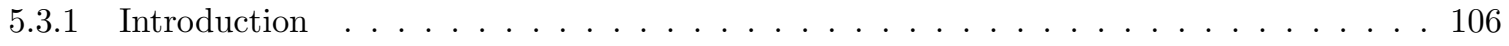

5.3.2 Liquid Film Entrainment/De-entrainment . . . . . . . . . . . . . . . . . . 107

5.3 .3 Bottom Reflood Entrainment _ . . . . . . . . . . . . . . . . . . . . . . 109

5.4 Top Down Reflood Entrainment/De-Entrainment . . . . . . . . . . . . . . . . . . 110

5.4 .1 Crossflow De-Entrainment . . . . . . . . . . . . . . . . . . . . 112

5.4 .2 De-Entrainment in Area Changes . . . . . . . . . . . . . . . . . . . . . . . . 112

5.4 .3 De-Entrainment at Solid Surfaces . . . . . . . . . . . . . . . . . . . . . . 113

$5.4 .4 \quad$ Spacer Grid Droplet Breakup Model . . . . . . . . . . . . . . . . . . . . . 113

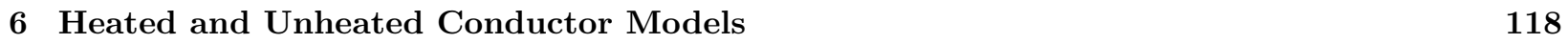

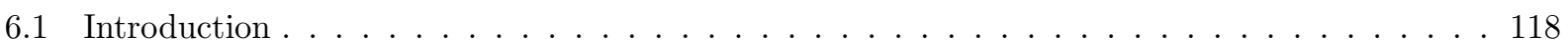

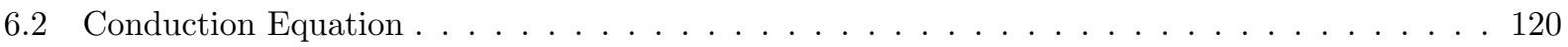


6.2.1 Formulation of the General Conduction Equation . . . . . . . . . . . . . . . . 120

6.2.2 Conductor and Flow Field Boundary . . . . . . . . . . . . . . . . . 127

6.2.2.1 Convective Heat Transfer . . . . . . . . . . . . . . . . . . . . . . 127

6.2.2.2 Droplet Impingement Heat Transfer . . . . . . . . . . . . . . . . . . . . . 128

6.2.3 Explicit Term Stability Criterion . . . . . . . . . . . . . . . . . . . . . 129

6.3 Conductor Re-Meshing . . . . . . . . . . . . . . . . . . . . 130

6.3.1 Re-Meshing Criteria . . . . . . . . . . . . . . . . . . . 132

6.4 Nuclear Fuel Rod Models . . . . . . . . . . . . . . . . . . . . . 136

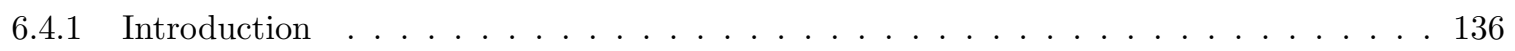

6.4 .2 Fuel Deformation Model . . . . . . . . . . . . . . . . . . . . . . 138

6.4.3 Dynamic Gap Conductance Model . . . . . . . . . . . . . . . . . . . . . . . . . . 143

6.4.3.1 Conduction Through the Fill Gas in the Gap . . . . . . . . . . . . . . . . . 143

6.4.3.2 Conduction in the Gap due to Pellet/Clad Contact . . . . . . . . . . . . 146

6.4.3.3 Radiative Heat Transfer in the Gap . . . . . . . . . . . . . . . . . . . . 146

6.4 Metal/Water Reaction . . . . . . . . . . . . . . . . . . . . 147

6.5 Departure from Nucleate Boiling (DNB) . . . . . . . . . . . . . . . . . . . 148

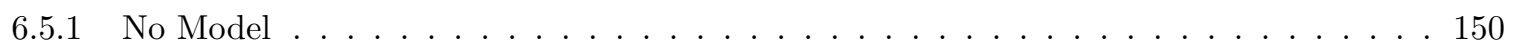

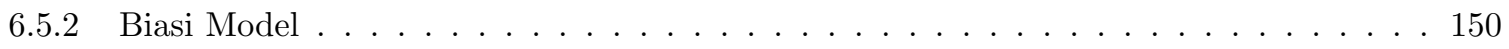

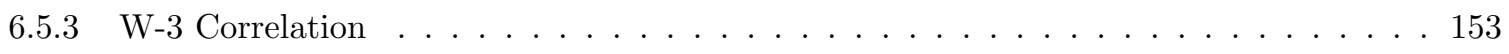

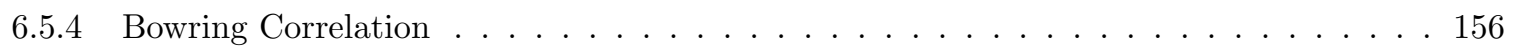

6.5.5 Groeneveld Look-up Tables . . . . . . . . . . . . . . . . . . . . . . 158

6.5.6 Calculation of $\boldsymbol{T}_{\mathrm{chf}} \ldots \ldots \ldots \ldots \ldots \ldots$

6.6 Heat Transfer Models . . . . . . . . . . . . . . . . . . . . . . . . . . 161

6.6 .1 Introduction $\ldots \ldots \ldots \ldots \ldots$

6.6.1.1 Minimum Film Boiling Temperature . . . . . . . . . . . . . . . 166

6.6.2 Wall Heat Transfer Closure Term . . . . . . . . . . . . . . . . . . . . . 167 
6.6.3 Single-Phase Vapor Convection . . . . . . . . . . . . . . . . . . . 169

6.6.4 Single-Phase Liquid Heat Transfer . . . . . . . . . . . . . . . . . . . . . . . . 170

6.6.5 Subcooled Nucleate Boiling ........................ . . 170

6.6.6 Saturated Nucleate Boiling . . . . . . . . . . . . . . . . . . . . . 174

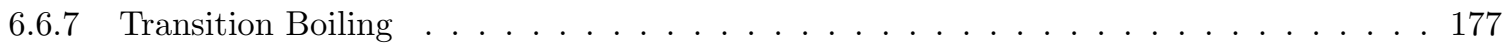

6.6.8 Inverted Annular Film Boiling . . . . . . . . . . . . . . . . . . . . 178

6.6.9 Dispersed Flow Film Boiling . . . . . . . . . . . . . . . . . . . . . . 181

6.6 .10 Radiative Heat Transfer . . . . . . . . . . . . . . . . . . . . . . . . . . . . 182

6.6.11 Grid Re-Wet Model . . . . . . . . . . . . . . . . . . . . . . . . . . 184

6.6.11.1 Dry Region Heat Balance . . . . . . . . . . . . . . . . . . . . 185

6.6.11.2 Wet Region Heat Balance . . . . . . . . . . . . . . . . . . 189

6.6.11.3 Grid Quench Front Model . . . . . . . . . . . . . . . . . . 191

7 Spacer Grid Models 194

7.1 Grid Heat Transfer Enhancement . . . . . . . . . . . . . . . . . . . . . . . . . . . . 194

8 Fluid Properties $\quad 196$

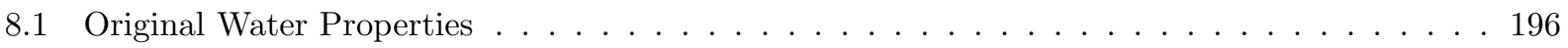

8.1.1 Saturated Liquid and Vapor . . . . . . . . . . . . . . . . . . . . 196

8.1 .2 Superheated Vapor . . . . . . . . . . . . . . . . . . . . 201

8.1.2.1 Enthalpy ........................... 201

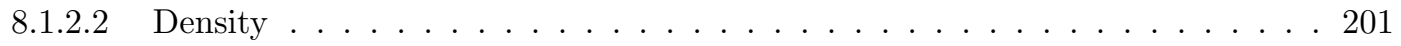

8.1.2.3 Temperature ............................ 202

8.1.2.4 Specific Heat . . . . . . . . . . . . . . . . . . . . 206

8.1.2.5 Thermal Conductivity ....................... 206

8.1.2.6 Viscosity ........................... 207

8.1.3 Subcooled Liquid . . . . . . . . . . . . . . . . . . . . . . . . . 207 
8.2 IAPWS Water Properties . . . . . . . . . . . . . . . . . . . . 209

8.3 Molten salt properties $\ldots \ldots \ldots \ldots \ldots \ldots \ldots$

9 Solid Properites $\quad 212$

9.1 Fuel Pellet Material Properties . . . . . . . . . . . . . . . . . . . . . . . . 212

$9.1 .1 \quad$ Fuel Emissivity . . . . . . . . . . . . . . . . . . . . . . . . . . 212

9.1 .2 Fuel Specific Heat Capacity . . . . . . . . . . . . . . . . . . . . . . . . 212

9.1 .3 Fuel Thermal Conductivity . . . . . . . . . . . . . . . . . . . . . . . . 214

9.1.4 Fuel Melting Temperature . . . . . . . . . . . . . . . . . . . . . 223

9.1.5 Fuel Thermal Expansion . . . . . . . . . . . . . . . . . . . . . . 225

9.2 Cladding Material Properties . . . . . . . . . . . . . . . . . . 227

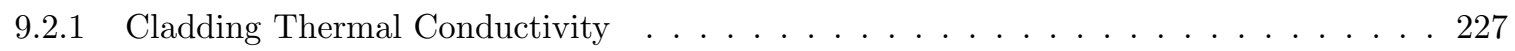

9.2 .2 Cladding Oxide Thermal Condutivity . . . . . . . . . . . . . . . 230

9.2 .3 Cladding Specific Heat . . . . . . . . . . . . . . . . . . . . . . . . . 231

9.2 .4 Cladding Surface Emissivity . . . . . . . . . . . . . . . . . . 233

9.2 .5 Cladding Meyer Hardness . . . . . . . . . . . . . . . . . . . . . . 233

9.2 .6 Cladding Thermal Expansion . . . . . . . . . . . . . . . . . . . 233

9.2.7 Cladding Elastic Modulus and Shear Modulus . . . . . . . . . . . . . . . . 236

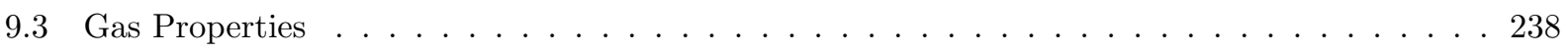

9.3 .1 Gas Conductivity . . . . . . . . . . . . . . . . . . . 238

9.3 .2 Molecular Weight . . . . . . . . . . . . . . . . . . . . . . 240

9.3 .3 Gas Specific Heat . . . . . . . . . . . . . . . . . . . . . . . . . . 240

9.3 .4 Gas Density . . . . . . . . . . . . . . . . . . . . . . . . . 242

9.3 .5 Gas Enthalpy . . . . . . . . . . . . . . . . . . . . . . . . 242

$\begin{array}{ll}\text { Bibliography } & 244\end{array}$

Consortium for Advanced Simulation of LWRs xii CASL-U-2018-1715-000 


\section{List of Figures}

2.1 Control volume defined in Cartesian coordinates $\ldots \ldots \ldots \ldots \ldots$

2.2 Scalar mesh cell and axial momentum mesh cell configuration . . . . . . . . . . . . . . . 14

2.3 Scalar mesh cell and transverse momentum mesh cell configuration . . . . . . . . . . . . . 14

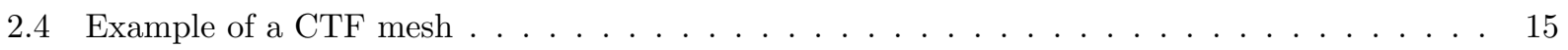

2.5 Spatial Notation Used to Demonstrate the Modified Godunov Scheme, by Ozdemir (2012) [6]. 47

2.6 Boric Acid Solubility Correlation w.r.t. $100 g$ water, by $\operatorname{Kim}(2008)[18] \ldots \ldots$

2.7 CTF Maximum Boric Acid Concentration by Kim et al.'s Correlation [18] . . . . . . . . . 52

3.1 Normal wall flow regimes recognized by $\mathrm{CTF} \ldots \ldots \ldots \ldots \ldots \ldots$

3.2 Normal wall flow regime selection $\operatorname{logic} \ldots \ldots \ldots \ldots \ldots \ldots$

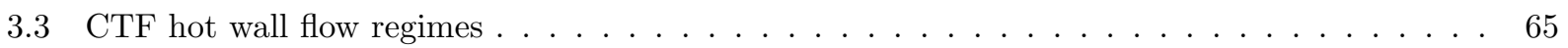

3.4 CTF hot wall flow regime selection $\operatorname{logic} \ldots \ldots \ldots \ldots \ldots \ldots$

4.1 Example of meshing of two adjacent sub-channels, $i i$ and $j j \ldots \ldots \ldots \ldots \ldots$

4.2 Behavior of the Beus two-phase mixing parameter $\ldots \ldots \ldots \ldots 6$

5.1 Ratio of shattered droplet diameter to incident droplet diameter dependence on droplet Weber number . . . . . . . . . . . . . . . . . . . . . . . . 115

5.2 Flowchart for the spacer grid droplet breakup model found in the GRID subroutine . . . . . 116

6.1 Flowchart for heated and unheated conductor temperature solution . . . . . . . . . . 119 
6.2 General CTF meshing approach for nuclear fuel $\operatorname{rod} \ldots \ldots \ldots \ldots$. . . . . . . . . 121

6.3 A closer look at CTF internal meshing of nuclear fuel $\operatorname{rod} \ldots \ldots \ldots$. . . . . . . . . 122

6.4 CTF model for fuel rods as a thermal resistance network . . . . . . . . . . . . . . . 125

6.5 Example of resistance in the axial direction for nuclear fuel $\operatorname{rod} \ldots \ldots \ldots \ldots$

6.6 Example of how a conductor is meshed based on adjoining fluid mesh $\ldots \ldots \ldots$. . . . . 131

6.7 Example of conductor mesh cell refinement for the first and second cells of a conductor . . . . 132

6.8 CTF procedure for determining whether conductor cells should be split, merged, or left alone 134

6.9 Flowchart of subroutine calls in the TEMP routine $\ldots \ldots \ldots \ldots \ldots$

6.10 Illustration of fuel pellet cracking $\ldots \ldots \ldots \ldots \ldots \ldots \ldots$

6.11 Illustration of the temperature jump near fuel pellet and clad surfaces in a fuel rod gap . . . 144

6.12 Boiling Curve . . . . . . . . . . . . . . . . . . . . . . . . 149

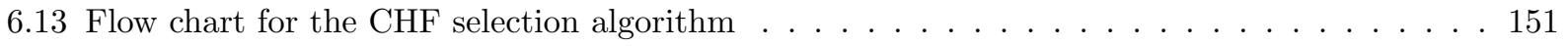

$6.14 \mathrm{CTF}$ algorithm for determining the temperature at $\mathrm{CHF} \ldots \ldots \ldots \ldots$

6.15 A simple example of how $\mathrm{T}_{\mathrm{CHF}}$ is iteratively calculated $\ldots \ldots \ldots \ldots$

$6.16 \mathrm{CTF}$ selection algorithm for the heat transfer regime $\ldots \ldots \ldots \ldots \ldots$

6.17 Model of rod and grid for calculating radiative heat transfer to grid $\ldots \ldots \ldots \ldots$

6.18 Grid-to-rod radiative heat transfer resistance network $\ldots \ldots \ldots \ldots$

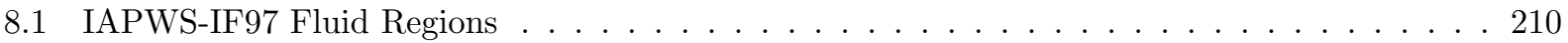

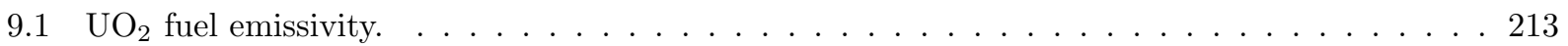

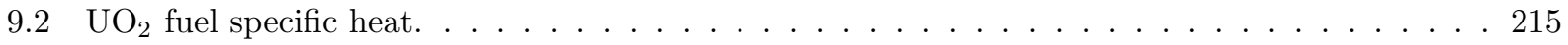

9.3 MATPRO-11 model for the thermal conductivity of $95 \%$ theoretical density (TD) $\mathrm{UO}_{2} \ldots \ldots 216$

9.4 Modified Nuclear Fuel Industries (NFI) model for the thermal conductivity of $95 \% \mathrm{TD} \mathrm{UO}_{2} .218$

9.5 Halden model for the thermal conductivity of $95 \% \mathrm{TD} \mathrm{UO}_{2} \ldots \ldots \ldots \ldots \ldots$

9.6 Duriez/Modified NFI model for the thermal conductivity of $95 \%$ TD MOX. . . . . . . . . 221

9.7 Halden model for the thermal conductivity of $95 \%$ TD MOX . . . . . . . . . . . . . 222 
9.8 Amaya model for the thermal conductivity of $95 \%$ TD MOX. . . . . . . . . . . . . . 224

9.9 Solidus and liquidus boundaries of the melting temperature (top) and melting temperature

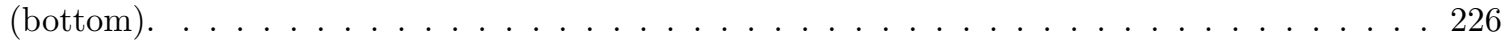

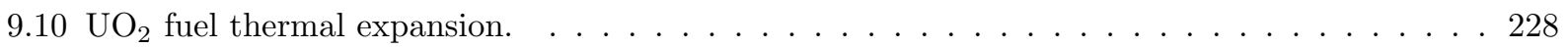

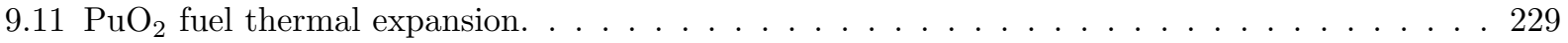

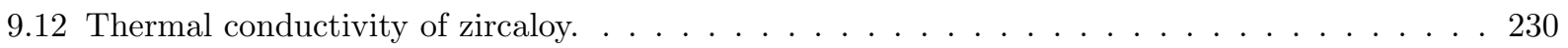

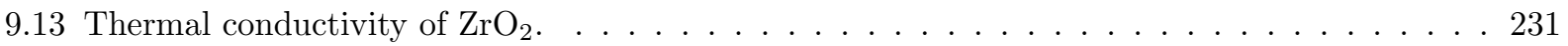

9.14 Cladding specific heat capacity. . . . . . . . . . . . . . . . . . . 232

9.15 Zirconium cladding emissivity (Equation 9.23$) \ldots \ldots \ldots \ldots \ldots \ldots$

9.16 Meyer hardness of cladding. . . . . . . . . . . . . . . . . . 235

9.17 Cladding axial and radial thermal expansion. . . . . . . . . . . . . . 236

9.18 Cladding elastic moduli for isotropic material. . . . . . . . . . . . . . . . . 239

9.19 Fill gas thermal conductivity. . . . . . . . . . . . . . . . . . . . . . . 240

9.20 Specific heat of air, nitrogen, hydrogen and oxygen. . . . . . . . . . . . . . . 243 


\section{Acronyms}

BWR boiling water reactor

CHF critical heat flux

COBRA-TF Coolant-Boiling in Rod Arrays-Two Fluids

CTF PSU RDFMG version of COBRA-TF

DNB departure from nucleate boiling

IAPWS International Association for the Properties of Water and Steam

LHS left-hand side

LOCA loss-of-coolant accident

LWR light water reactor

NFI Nuclear Fuel Industries

NPP nuclear power plant

NRC Nuclear Regulatory Commission

ONB onset of nucleate boiling

PSU Pennsylvania State University

PWR pressurized water reactor

RDFMG Reactor Dynamics and Fuel Modeling Group

RHS right-hand side

SIMPLE Semi-Implicit Method for Pressure-Linked Equations

TD theoretical density

$\mathbf{T} / \mathbf{H}$ thermal/hydraulic

$\mathbf{V} \& \mathbf{V}$ validation \& verification 


\section{Introduction}

Perhaps the most important aspect of the civilian use of nuclear power is ensuring public safety from radiological releases despite any number of postulated risks to the integrity of nuclear power plant (NPP) systems. Such insurance is obtained through rigorous testing and re-testing of plant safety systems for any and all likely accident scenarios that can threaten NPP stability. Accident behavior is best assessed through a combination of experimental and computational simulations of postulated reactor accident scenarios. With 1988 revisions to Nuclear Regulatory Commission (NRC) safety analysis requirements (10CFR.50.46), great gains in plant economy as well as safety can be made with the use of computational best-estimate models in plant design and operation.

COBRA-TF is one such computational tool for assessing NPP behavior. The code, developed originally in 1980 by Pacific Northwest Laboratory under sponsorship of the NRC [1], began as a T/H rod-bundle analysis code, but has been continually updated and expanded over the past several decades. The code uses a twofluid modeling approach with consideration for three separate, independent flow fields; fluid film, vapor, and liquid droplets. COBRA-TF includes a wide range of $\mathrm{T} / \mathrm{H}$ models crucial to accurate LWR safety analysis including, but not limited to, flow regime dependent two-phase heat transfer, inter-phase heat transfer and drag, droplet breakup, and quench-front tracking. Due to its full 3D capabilities and extensive array of reactor $\mathrm{T} / \mathrm{H}$ modeling capabilities, COBRA-TF has found much use in modeling of LWR rod-bundle transient analysis and pressurized water reactor (PWR) whole-vessel loss-of-coolant accident (LOCA) analysis.

CTF is an improved version of COBRA-TF developed and maintained by the RDFMG at the PSU. Improvements include:

- Transition to FORTRAN 90 source code

- Enhanced user-friendliness with improved error checking and free-format input deck

- Quality assurance utilizing an extensive validation \& verification (V\&V) matrix

- Turbulent mixing, void drift and direct heating model improvements

- Enhanced computational efficiency by implementation of new numerical solution schemes

- Better code physical model and user modeling documentation

This document discusses, in detail, the numerical solution and physical phenomena modeling capabilities of CTF. It is, in essence, a theory manual for the code. The two-fluid modeling approach, associated 
conservation equations and numerical solution process is first discussed in Chapter 2. This is the foundation for modeling flow — setting up the transport equations for each unique fluid, discretizing them, applying them to a mesh of the volume, and solving them by a numerical iterative approach.

Setting up the conservation equations is only the first step and they cannot be solved until they are completely defined. The equations will contain several terms that link similar conservation equations located in different spatial locations (i.e. mesh cells) due to physical phenomena like void drift and turbulent mixing. Likewise, they will have terms that link equations in the same spatial location due to inter-phase effects like phase change and entrainment. These terms are dubbed "closure" terms because they define the remaining conservation equation terms and allow them to be solved for the independent variables. There are two general types of these closure terms: field interactions that arise from outside of the mesh cell that a given conservation equation is defined in or field interactions that arise from within the mesh cell that the given conservation equation is defined for.

Prior to modeling these micro- and macro-cell interactions, it is necessary to determine the general behavior of the two-phase flow in the mesh cell being modeled. This is done by using a flow regime map to categorize the flow behavior into one of several classifications of flow. This allows for accurate determination of the inter-phase contact area, which is necessary for determination of inter-phase heat transfer and drag, as well as the correct selection of closure-term models. The CTF flow regime maps and inter-phase area calculation process are described in Chapter 3.

Chapter 4 then discusses the "macro-interaction" models like wall drag, wall heat transfer, turbulent mixing, and void drift. Chapter 5 disscusses the "micro-interaction" models like inter-phase mass transfer and interphase drag.

The only remaining unknown parameters at this point are the thermophysical fluid and solid properties. These properties are available to CTF via intrinsic fluid property data tables and are discussed in Chapters 8 and 9 .

With this information and adequete user-provided boundary conditions, CTF is capable of deriving a solution for fluid behavior; however, it may be desireable to also model the behavior of fuel rods or reactor decay heat due to their importance in safety analysis. CTF also includes models for heated and unheated conductors, including nuclear fuel rod models, which are discussed in Chapter 6.

The primary purpose of this manual is the discussion of the theory behind CTF. However, some mention is also made of the actual source code implementation of the aformentioned models. Additional documentation outside of this manual are also available for describing instructions for modeling T/H problems using CTF as well as for providing details on modules and global variables in CTF source code. 


\section{Conservation Equations and Numer- ical Solution}

\section{$2.1 \quad$ Introduction}

This chapter lays the foundation in describing the CTF flow modeling approach through discussion of the code conservation equations. The equations are presented in their most basic forms and they are gradually manipulated into the forms that were used for the creation of COBRA-TF.

A two-fluid model is employed in CTF with consideration for three separate fluid fields - liquid film, liquid droplets, and vapor. Each of the three fields is modeled with its own set of conservation equations with the exception being that the liquid and droplet fields are assumed to be in thermal equilibrium and, thus, they share an energy equation. The sets of conservation equations are formulated either using a Cartesian coordinate system (3-D solution) or using a simplified sub-channel approach. The user may choose which approach to use when modeling a system with CTF. The equations are solved simultaneously using the Semi-Implicit Method for Pressure-Linked Equations (SIMPLE).

In this chapter, the general conservation equations of mass, momentum and energy are first presented in Section 2.2. Assumptions are listed and the equations are reduced where necessary. They are then expanded out for each field and for each spatial direction.

To be useful in a $\mathrm{T} / \mathrm{H}$ code environment, the conservation equations must be put into finite-difference form and then applied and solved over a mesh of finite volumes. The computational mesh structure and discretized forms of the conservation equations are discussed in Section 2.3. Once the governing conservation equations are set up for a computational cell mesh of the flow geometry, they must be solved simultaneously using a numerical approach. The numerical approach is discussed in Section 2.4. 


\subsection{Conservation Equations}

\subsubsection{Introduction}

The generalized form of the conservation equations of mass, momentum, and energy are given first with a description of each term in the equations in Section 2.2.2. After each conservation equation is presented, assumptions are defined that are used for implementing the equations into CTF. Next, the Cartesian forms of the CTF conservation equations are shown in Section 2.2.3 and the sub-channel forms of the CTF conservation equations are shown in Section 2.2.4.

\subsubsection{Generalized Conservation Equations}

For a given computational cell, mass, momentum, and energy are conserved. This behavior is captured for each field using three conservation equations (with the exception of the liquid and droplet fields sharing an energy equation). This is the heart of the two-fluid model — modeling each phase with its own set of mass, momentum, and energy equations. The conservation equations, of course, are dependent on one another and are linked by interaction terms that account for things like mass and heat transfer between phases (e.g. evaporation/condensation or entrainment/de-entrainment). Each of the conservation equations is presented and discussed in succession with a description of each term in the equations.

\subsubsection{Generalized Phasic Mass Conservation Equation}

The general mass conservation equation is presented in Equation 2.1.

$$
\frac{\partial}{\partial t}\left(\alpha_{k} \rho_{k}\right)+\nabla \cdot\left(\alpha_{k} \rho_{k} \vec{V}_{k}\right)=L_{k}+M_{e}^{T}
$$

The $k$ subscript denotes the field under consideration; it can be $l$ for the liquid film field, $v$ for the vapor field, or $e$ for the entrained droplet field. On the left-hand side (LHS) of Equation 2.1, the first term is the change of mass with time and the second term is the advection of the field mass into or out of the volume ( $\vec{V}$ is the field velocity). On the right-hand side (RHS), the $L_{k}$ term represents the mass transfer into or out of phase $k$ - inter-phase mass transfer can occur by either evaporation/condensation or by entrainment/ de-entrainment. The $L$ term is expanded for each field as follows:

$$
\begin{aligned}
& L_{v}=\Gamma^{\prime \prime \prime} \\
& L_{l}=-(1-\eta) \Gamma^{\prime \prime \prime}-S^{\prime \prime \prime}, \text { and } \\
& L_{e}=-\eta \Gamma^{\prime \prime \prime}+S^{\prime \prime \prime}
\end{aligned}
$$

The $\Gamma^{\prime \prime \prime}$ term represents the volumetric mass transfer due to phase change. Note that the $\Gamma^{\prime \prime \prime}$ term is additive for the vapor field whereas it is subtractive for the liquid and droplet fields — if the sign of $\Gamma^{\prime \prime \prime}$ is 
positive, evaporation adds mass to the vapor and subtracts it from the liquid fields (the reverse is true for condensation). For the liquid and droplet fields, $\Gamma^{\prime \prime \prime}$ is multiplied by $1-\eta$ and $\eta$, respectively. The $\eta$ term represents the fraction of phase change occurring between vapor and entrained droplets. Equation 2.3 shows the definition of $\eta$ for the case of evaporation while Equation 2.4 shows the definition of $\eta$ for the case of condensation.

$$
\begin{gathered}
\eta_{\text {evap }}=\min \left\{\begin{array}{l}
1-\frac{Q_{w l}^{\prime \prime \prime}}{\Gamma^{\prime \prime \prime} H_{f g}} \\
\frac{\alpha_{e}}{1-\alpha_{v}}
\end{array}\right. \\
\eta_{\text {cond }}=\frac{\alpha_{e}}{1-\alpha_{v}}
\end{gathered}
$$

In Equations 2.3 and 2.4, $Q_{w l}^{\prime \prime \prime}$ is the volumetric heat transfer from the wall to liquid phase and $H_{f g}$ is the latent heat of vaporization. The phase change model used for the calculation of $\Gamma$ along with the entrainment/de-entrainment models are described in Chapter 5.

The final term of the general mass conservation equation is the mass transfer in the mesh cell due to turbulent mixing and void drift. Advanced turbulence models are not included in CTF and because axially-dominated sub-channel flow is assumed, a simple turbulent diffusion model is used to calculated turbulent transfer of axial momentum through sub-channel gaps. Note that turbulent mixing only occurs in the lateral direction. The turbulent diffusion approximation and void drift models are further discussed in Chapter 4 .

\subsubsection{Generalized Phasic Momentum Conservation Equation}

The momentum conservation equation is given in Equation 2.5.

$$
\begin{aligned}
& \frac{\partial}{\partial t}\left(\alpha_{k} \rho_{k} \vec{V}_{k}\right)+\frac{\partial}{\partial x}\left(\alpha_{k} \rho_{k} u_{k} \vec{V}_{k}\right)+\frac{\partial}{\partial y}\left(\alpha_{k} \rho_{k} v_{k} \vec{V}_{k}\right)+\frac{\partial}{\partial z}\left(\alpha_{k} \rho_{k} w_{k} \vec{V}_{k}\right) \\
& \quad=\alpha_{k} \rho_{k} \vec{g}-\alpha_{k} \nabla P+\nabla \cdot\left[\alpha_{k}\left(\tau_{k}^{i j}+\boldsymbol{T}_{k}^{i j}\right)\right]+\vec{M}_{k}^{L}+\vec{M}_{k}^{d}+\vec{M}_{k}^{T}
\end{aligned}
$$

On the LHS, the terms are change of volume momentum with time and advection of momentum. Note that the LHS terms multiply by the vector velocity $\vec{V}_{k}$ and so each term will have three components $\left(u_{k} \vec{i}+v_{k} \vec{j}+\right.$ $w_{k} \vec{k}$ ) and result in three separate momentum equations for each of the three directions (if using Cartesian coordinates). On the RHS, the terms are: gravitational force, pressure force, viscous and turbulent shear stress, momentum source/sink due to phase change and entrainment, interfacial drag forces, and momentum transfer due to turbulent mixing. The pressure has no phase dependency because it is assumed to be equal in all phases and gravity is assumed to be the only body force.

The turbulent shear stress term is actually not modeled in CTF, so it is eliminated in future forms of the momentum equation. Turbulent mixing is captured using a simple turbulent diffusion approximation.

The viscous stress term can be expanded into wall shear and fluid-fluid shear components, as follows: 


$$
\begin{aligned}
& \nabla \cdot\left(\alpha_{e} \boldsymbol{\tau}_{e}^{i j}\right)=\vec{\tau}_{\text {we }}^{\prime \prime \prime} \\
& \nabla \cdot\left(\alpha_{v} \boldsymbol{\tau}_{v}^{i j}\right)=\vec{\tau}_{\mathrm{wv}}^{\prime \prime \prime}+\nabla \cdot\left(\alpha_{v} \boldsymbol{\sigma}_{v}^{i j}\right) \\
& \nabla \cdot\left(\alpha_{l} \boldsymbol{\tau}_{l}^{i j}\right)=\vec{\tau}_{\mathrm{wl}}^{\prime \prime \prime}+\nabla \cdot\left(\alpha_{l} \boldsymbol{\sigma}_{l}^{i j}\right)
\end{aligned}
$$

The $\vec{\tau}_{\text {we }}^{\prime \prime \prime}, \vec{\tau}_{\text {wv }}^{\prime \prime \prime}$, and $\vec{\tau}_{\text {wl }}^{\prime \prime \prime}$ terms are the volumetric wall drag and form losses of the entrained, vapor, and liquid phases, respectively. Note that the entrained droplet field is assumed not to contact the wall and, thus, there is no wall drag; however, the $\vec{\tau}_{\text {we }}^{\prime \prime \prime}$ term does account for form loss effects experienced by the droplet field. The liquid-liquid viscous shear stresses actually aren't modeled by CTF and so they are eliminated in future derivations of the momentum equations.

The $\vec{M}_{k}^{L}$ term is the momentum source/sink due to phase change and entrainment/de-entrainment — it is expanded as follows:

$$
\begin{aligned}
& \vec{M}_{v}^{L}=\Gamma^{\prime \prime \prime} \vec{V} \\
& \vec{M}_{l}^{L}=-\Gamma^{\prime \prime \prime}(1-\eta) \vec{V}-S^{\prime \prime \prime} \vec{V} \\
& \vec{M}_{e}^{L}=-\Gamma^{\prime \prime \prime} \eta \vec{V}+S^{\prime \prime \prime} \vec{V}
\end{aligned}
$$

The $\vec{V}$ term is the velocity of the phase that mass is coming from. For example, if evaporation is occurring from the droplet field then the momentum, $\Gamma^{\prime \prime \prime} \eta \vec{V}_{e}$, will leave entrained field and be subtracted from the droplet field momentum conservation equation. Conversely, that same amount of momentum will enter the vapor field.

The interfacial drag term, $\vec{M}^{d}$, can simply be expanded for each of the three fields as follows:

$$
\begin{aligned}
& \vec{M}_{v}^{d}=-\vec{\tau}_{\mathrm{i}, \mathrm{vl}}^{\prime \prime \prime}-\vec{\tau}_{\mathrm{i}, \mathrm{ve}}^{\prime \prime \prime} \\
& \vec{M}_{l}^{d}=\vec{\tau}_{\mathrm{i}, \mathrm{vl}}^{\prime \prime \prime} \\
& \vec{M}_{e}^{d}=\vec{\tau}_{\mathrm{i}, \mathrm{ve}}^{\prime \prime \prime}
\end{aligned}
$$

The $\vec{\tau}_{\mathrm{i}, \mathrm{vl}}^{\prime \prime \prime}$ and $\vec{\tau}_{\mathrm{i}, \mathrm{ve}}^{\prime \prime \prime}$ terms are the volumetric inter-phase drag forces for the vapor-liquid and vapor-droplet interfaces, respectively. Note that the drag terms are subtractive for the vapor field and additive for the droplet and liquid fields. This is because the CTF convention is for the vapor phase to move faster than liquid and droplet phases, which means that interfacial friction would act against the vapor phase, but with the liquid and droplet phases. If the opposite were true, and the vapor phase were somehow moving slower than the liquid and droplet phases, the signs of the terms would simply be reversed in CTF. The interfacial shear is a flow regime-dependent value and its calculation is further discussed in Chapter 5.

The final term of Equation 2.5 is the momentum source due to turbulent mixing and void drift, $\vec{M}_{k}^{T}$. Note that only axial momentum is convected by turbulent mixing and void drift. 

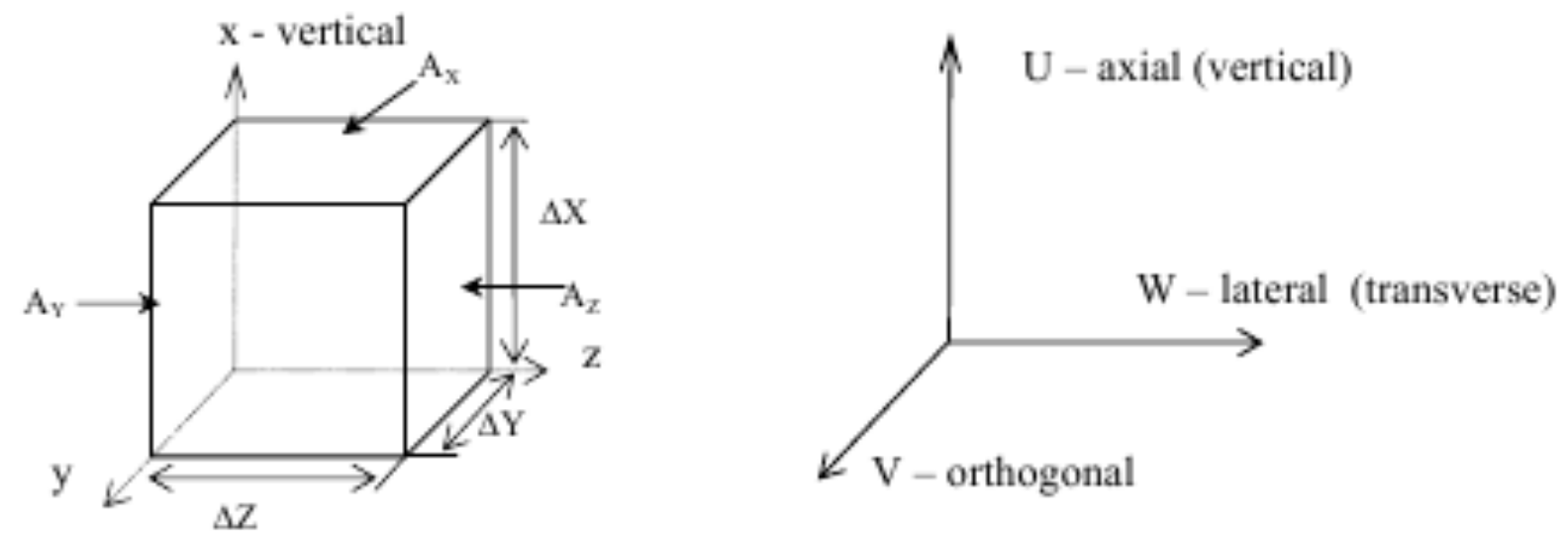

Figure 2.1: Control volume defined in Cartesian coordinates

\subsubsection{Generalized Phasic Energy Conservation Equation}

The generalized energy equation is presented in Equation 2.11.

$$
\begin{array}{r}
\frac{\partial}{\partial t}\left(\alpha_{k} \rho_{k} h_{k}\right)+\nabla \cdot\left(\alpha_{k} \rho_{k} h_{k} \vec{V}_{k}\right)=-\nabla \cdot\left[\alpha_{k}\left(\vec{Q}_{k}+\vec{q}_{k}^{T}\right)\right]+ \\
\Gamma_{k} h_{k}^{i}+q_{w k}^{\prime \prime \prime}+\alpha_{k} \frac{\partial P}{\partial t}
\end{array}
$$

The LHS terms are change of phase energy with respect to time and advection of phase energy into or out of the cell. The RHS terms are: $k$-phase conduction and turbulence heat flux, energy transfer due to phasechange, volumetric wall heat transfer, and the pressure work term. It is assumed that there is no volumetric heat generation occurring in the fluid, radiative heat transfer only occurs between solid surfaces and the vapor/droplet fields, internal dissipation is negligible, and, once again, pressure is uniform throughout the phases.

There is actually no modeling of heat conduction in the fluids and so $\vec{Q}_{k}$ is zero in CTF. The $\vec{q}_{k}^{T}$ term represents energy exchange by both turbulent mixing and by void drift; however, it is only considered in the lateral and orthogonal directions.

\subsubsection{CTF Expanded Conservation Equations in Cartesian Form}

The generalized conservation equations are presented in their expanded form in this section with consideration for the aforementioned assumptions. Each field conservation equation is explicitly written out. Figure 2.1 shows a control volume defined for the Cartesian geometry[2]. 


\subsubsection{Mass Continuity Conservation Equation in Cartesian Coordinates}

Vapor conservation of mass is shown in Equation 2.12.

$$
\frac{\partial}{\partial t}\left(\alpha_{v} \rho_{v}\right)+\frac{\partial}{\partial x}\left(\alpha_{v} \rho_{v} u_{v}\right)+\frac{\partial}{\partial y}\left(\alpha_{v} \rho_{v} v_{v}\right)+\frac{\partial}{\partial z}\left(\alpha_{v} \rho_{v} w_{v}\right)=\Gamma^{\prime \prime \prime}+M_{v}^{T^{\prime \prime \prime}}
$$

Liquid conservation of mass is shown in Equation 2.13.

$$
\frac{\partial}{\partial t}\left(\alpha_{l} \rho_{l}\right)+\frac{\partial}{\partial x}\left(\alpha_{l} \rho_{l} u_{l}\right)+\frac{\partial}{\partial y}\left(\alpha_{l} \rho_{l} v_{l}\right)+\frac{\partial}{\partial z}\left(\alpha_{l} \rho_{l} w_{l}\right)=-\Gamma^{\prime \prime \prime}(1-\eta)-S^{\prime \prime \prime}+M_{l}^{T^{\prime \prime \prime}}
$$

Droplet conservation of mass is shown in Equation 2.14.

$$
\frac{\partial}{\partial t}\left(\alpha_{e} \rho_{l}\right)+\frac{\partial}{\partial x}\left(\alpha_{e} \rho_{l} u_{e}\right)+\frac{\partial}{\partial y}\left(\alpha_{e} \rho_{l} v_{e}\right)+\frac{\partial}{\partial z}\left(\alpha_{e} \rho_{l} w_{e}\right)=-\Gamma^{\prime \prime \prime} \eta+S^{\prime \prime \prime}+M_{e}^{T^{\prime \prime \prime}}
$$

\subsubsection{Momentum Conservation Equation in Cartesian Coordinates}

Each field can move in three separate directions in Cartesian coordinates, so there is an $x$-, $y$-, and $z$ component of each field's momentum conservation equation. The vapor conservation equation for the $x$ direction is given in Equation 2.15.

$$
\begin{aligned}
& \frac{\partial}{\partial t}\left(\alpha_{v} \rho_{v} u_{v}\right)+\frac{\partial}{\partial x}\left(\alpha_{v} \rho_{v} u_{v} u_{v}\right)+\frac{\partial}{\partial y}\left(\alpha_{v} \rho_{v} u_{v} v_{v}\right)+\frac{\partial}{\partial z}\left(\alpha_{v} \rho_{v} u_{v} w_{v}\right) \\
& \quad=-\alpha_{v} \frac{\partial P}{\partial x}-\alpha_{v} \rho_{v} g-\tau_{\mathrm{wx}, \mathrm{v}}^{\prime \prime \prime}-\tau_{\mathrm{ix}, \mathrm{vl}}^{\prime \prime \prime}-\tau_{\mathrm{ix}, \mathrm{ve}}^{\prime \prime \prime}+\Gamma^{\prime \prime \prime} u+\sum_{k=1}^{n k}\left(M_{v}^{T}\right)_{k}^{\prime \prime \prime}
\end{aligned}
$$

The axial component of momentum can be convected into the mesh cell from any of three directions, which accounts for the three advection terms on the LHS of Equation 2.15. The three shear terms only include shear forces acting on the vapor phase in the $x$-direction. The momentum source from mass transfer, $\Gamma^{\prime \prime \prime} u$, will have components of phase change from both the droplet and liquid fields and the phase velocity that multiplies by $\Gamma^{\prime \prime \prime}$ will be dependent on the direction of phase change (i.e. evaporation or condensation). The final term accounts for turbulent mixing of axial momentum - it is summed over all transverse connections to the mesh cell.

The $y$ - and $z$ - components of vapor momentum conservation are shown in Equations 2.16 and 2.17, respectively. Note, there are no gravitational body force terms in the $y$ - and $z$-component equations and no turbulent mixing of transverse momentum. 


$$
\begin{aligned}
& \frac{\partial}{\partial t}\left(\alpha_{v} \rho_{v} v_{v}\right)+\frac{\partial}{\partial x}\left(\alpha_{v} \rho_{v} v_{v} u_{v}\right)+\frac{\partial}{\partial y}\left(\alpha_{v} \rho_{v} v_{v} v_{v}\right)+\frac{\partial}{\partial z}\left(\alpha_{v} \rho_{v} v_{v} w_{v}\right)=-\alpha_{v} \frac{\partial P}{\partial y}-\tau_{\mathrm{w}, \mathrm{v}, \mathrm{v}, \tau_{\mathrm{i}, \mathrm{vl}}^{\prime \prime \prime}-\tau_{\mathrm{i}, \mathrm{ve}}^{\prime \prime \prime}+\Gamma^{\prime \prime \prime} v \quad(2.16)} \quad\left(\alpha^{\prime \prime}{ }^{\prime \prime}\left(\alpha_{v} \rho_{v} w_{v}\right)+\frac{\partial}{\partial x}\left(\alpha_{v} \rho_{v} w_{v} u_{v}\right)+\frac{\partial}{\partial y}\left(\alpha_{v} \rho_{v} w_{v} v_{v}\right)+\frac{\partial}{\partial z}\left(\alpha_{v} \rho_{v} w_{v} w_{v}\right)=-\alpha_{v} \frac{\partial P}{\partial z}-\tau_{\mathrm{wz}, \mathrm{v}}^{\prime \prime \prime}-\tau_{\mathrm{iz}, \mathrm{vl}}^{\prime \prime \prime}-\tau_{\mathrm{iz}, \mathrm{ve}}^{\prime \prime \prime}+\Gamma^{\prime \prime \prime} w\right.
\end{aligned}
$$

The liquid conservation equations are given in Equations 2.18, 2.19, and 2.20.

$$
\begin{gathered}
\frac{\partial}{\partial t}\left(\alpha_{l} \rho_{l} u_{l}\right)+\frac{\partial}{\partial x}\left(\alpha_{l} \rho_{l} u_{l} u_{l}\right)+\frac{\partial}{\partial y}\left(\alpha_{l} \rho_{l} u_{l} v_{l}\right)+\frac{\partial}{\partial z}\left(\alpha_{l} \rho_{l} u_{l} w_{l}\right) \\
=-\alpha_{l} \frac{\partial P}{\partial x}-\alpha_{l} \rho_{l} g-\tau_{\mathrm{wx}, 1}^{\prime \prime \prime}+\tau_{\mathrm{ix}, \mathrm{vl}}^{\prime \prime \prime}-\Gamma^{\prime \prime \prime}(1-\eta) u+\sum_{k=1}^{n k}\left(M_{l}^{T}\right)_{k}^{\prime \prime \prime} \\
\frac{\partial}{\partial t}\left(\alpha_{l} \rho_{l} v_{l}\right)+\frac{\partial}{\partial x}\left(\alpha_{l} \rho_{l} v_{l} u_{l}\right)+\frac{\partial}{\partial y}\left(\alpha_{l} \rho_{l} v_{l} v_{l}\right)+\frac{\partial}{\partial z}\left(\alpha_{l} \rho_{l} v_{l} w_{l}\right)=-\alpha_{l} \frac{\partial P}{\partial y}-\tau_{\mathrm{wy}, \mathrm{l}}^{\prime \prime \prime}+\tau_{\mathrm{iy}, \mathrm{vl}}^{\prime \prime \prime}-\Gamma^{\prime \prime \prime}(1-\eta) v \\
\frac{\partial}{\partial t}\left(\alpha_{l} \rho_{l} w_{l}\right)+\frac{\partial}{\partial x}\left(\alpha_{l} \rho_{l} w_{l} u_{l}\right)+\frac{\partial}{\partial y}\left(\alpha_{l} \rho_{l} w_{l} v_{l}\right)+\frac{\partial}{\partial z}\left(\alpha_{l} \rho_{l} w_{l} w_{l}\right)=-\alpha_{l} \frac{\partial P}{\partial z}-\tau_{\mathrm{wz}, \mathrm{l}}^{\prime \prime \prime}+\tau_{\mathrm{iz}, \mathrm{vl}}^{\prime \prime \prime}-\Gamma^{\prime \prime \prime}(1-\eta) w
\end{gathered}
$$

Note in the liquid momentum equations that the interfacial shear components became additive while the momentum source due to mass transfer became subtractive.

Equations 2.21-2.23 show the $x$-, $y$ - and $z$-components of momentum conservation for the entrained field.

$$
\begin{gathered}
\frac{\partial}{\partial t}\left(\alpha_{e} \rho_{l} u_{e}\right)+\frac{\partial}{\partial x}\left(\alpha_{e} \rho_{l} u_{e} u_{e}\right)+\frac{\partial}{\partial y}\left(\alpha_{e} \rho_{l} u_{e} v_{e}\right)+\frac{\partial}{\partial z}\left(\alpha_{e} \rho_{l} u_{e} w_{e}\right) \\
=-\alpha_{e} \frac{\partial P}{\partial x}-\alpha_{e} \rho_{l} g-\tau_{\mathrm{wx}, \mathrm{e}}^{\prime \prime \prime}+\tau_{\mathrm{ix}, \mathrm{ve}}^{\prime \prime \prime}-\Gamma^{\prime \prime \prime} \eta u+\sum_{k=1}^{n k}\left(M_{e}^{T}\right)_{k}^{\prime \prime \prime} \\
\frac{\partial}{\partial t}\left(\alpha_{e} \rho_{l} v_{e}\right)+\frac{\partial}{\partial x}\left(\alpha_{e} \rho_{l} v_{e} u_{e}\right)+\frac{\partial}{\partial y}\left(\alpha_{e} \rho_{l} v_{e} v_{e}\right)+\frac{\partial}{\partial z}\left(\alpha_{e} \rho_{l} v_{e} w_{e}\right)=-\alpha_{e} \frac{\partial P}{\partial y}-\tau_{\mathrm{wy}, \mathrm{e}}^{\prime \prime \prime}+\tau_{\mathrm{iy}, \mathrm{ve}}^{\prime \prime \prime}-\Gamma^{\prime \prime \prime} \eta v \\
\frac{\partial}{\partial t}\left(\alpha_{e} \rho_{l} w_{e}\right)+\frac{\partial}{\partial x}\left(\alpha_{e} \rho_{l} w_{e} u_{e}\right)+\frac{\partial}{\partial y}\left(\alpha_{e} \rho_{l} w_{e} v_{e}\right)+\frac{\partial}{\partial z}\left(\alpha_{e} \rho_{l} w_{e} w_{e}\right)=-\alpha_{e} \frac{\partial P}{\partial z}-\tau_{\mathrm{wz}, \mathrm{e}}^{\prime \prime \prime}+\tau_{\mathrm{iz}, \mathrm{ve}}^{\prime \prime \prime}-\Gamma^{\prime \prime \prime} \eta w
\end{gathered}
$$




\subsubsection{Energy Equation in Cartesian Coordinates}

There are only two energy equations required — one for vapor phase and one for the entrained and liquid fields. Equation 2.24 provides the vapor energy equation in Cartesian coordinates.

$$
\frac{\partial}{\partial t}\left(\alpha_{v} \rho_{v} h_{v}\right)+\frac{\partial}{\partial x}\left(\alpha_{v} \rho_{v} h_{v} u_{v}\right)+\frac{\partial}{\partial y}\left(\alpha_{v} \rho_{v} h_{v} v_{v}\right)+\frac{\partial}{\partial z}\left(\alpha_{v} \rho_{v} h_{v} w_{v}\right)=\frac{\partial}{\partial y} q_{v, y}^{T}+\frac{\partial}{\partial z} q_{v, z}^{T}+\Gamma^{\prime \prime \prime} h+q_{w v}^{\prime \prime \prime}+\alpha_{v} \frac{\partial P}{\partial t}
$$

The $q_{v}^{T}$ term is the transfer of energy into or out of the mesh cell due to turbulent exchange; it is calculated using a turbulent diffusion approximation. Enthalpy will be carried into or out of the mesh cell in the lateral directions only, hence the absence of the $x$-direction term. The $\Gamma^{\prime \prime \prime} h$ term is the implicit heat transfer term which accounts for evaporation and condensation effects. The enthalpy in the term will either be the saturation liquid or vapor enthalpy; however, no subscript has been provided in Equation 2.24 for $h$ because the implicit heat transfer model is slightly more complicated than it is depicted here, as it takes into account contributions of mass transfer from subcooled liquid, subcooled vapor, super-heated liquid and super-heated vapor. The enthalpy carried with $\Gamma^{\prime \prime \prime}$ will be dependent on the evaporating and condensing sources. The implicit heat transfer model is further discussed in greater detail in Chapter 5.

Equation 2.25 presents the liquid energy equation.

$$
\begin{gathered}
\frac{\partial}{\partial t}\left(\left(\alpha_{l}+\alpha_{e}\right) \rho_{l} h_{l}\right)+\frac{\partial}{\partial x}\left(\alpha_{l} \rho_{l} h_{l} u_{l}\right)+\frac{\partial}{\partial x}\left(\alpha_{e} \rho_{l} h_{l} u_{e}\right)+\frac{\partial}{\partial y}\left(\alpha_{l} \rho_{l} h_{l} v_{l}\right)+\frac{\partial}{\partial y}\left(\alpha_{e} \rho_{l} h_{l} v_{e}\right) \\
+\frac{\partial}{\partial z}\left(\alpha_{l} \rho_{l} h_{l} w_{l}\right)+\frac{\partial}{\partial z}\left(\alpha_{e} \rho_{l} h_{l} w_{e}\right)=\frac{\partial}{\partial y} q_{l, y}^{T}+\frac{\partial}{\partial z} q_{l, z}^{T}-\Gamma^{\prime \prime \prime} h+q_{w v}^{\prime \prime \prime}+\left(\alpha_{l}+\alpha_{e}\right) \frac{\partial P}{\partial t}
\end{gathered}
$$

Equation 2.25 includes six advection terms since both droplet and liquid fields are modeled. However, turbulent mixing and void drift only causes mixing of the liquid field.

\subsubsection{CTF Conservation Equations in Sub-Channel Form}

The sub-channel approach is a simplification of the conservation equations that only considers two flow directions - axial flow and lateral flow. The lateral flow directions are not a set of fixed coordinates; instead, the term, "lateral flow" covers any orthogonal direction to the vertical axis. Because fixed coordinates are not defined for the lateral direction in the sub-channel approach, lateral flow has no direction once it leaves a gap. Lateral flow enters a sub-channel volume through "gaps" between the volume and other adjacent sub-channel volumes. This is a suitable assumption for the axially-dominated flow of a reactor fuel bundle because the relatively minuscule lateral flows transfer little momentum across sub-channel mesh cell elements. The result of this assumption is one less momentum equation for each of the three fields. 


\subsubsection{Mass Conservation Equations in Sub-Channel Coordinates}

The vapor, liquid, and entrained droplet mass conservation equations are presented in Equations 2.26 - 2.28. The gap velocity is now represented by $w_{k}$. The gap mass transfer terms are summed over all the gaps connecting to the mesh cell.

$$
\begin{gathered}
\frac{\partial}{\partial t}\left(\alpha_{v} \rho_{v}\right)+\frac{\partial}{\partial x}\left(\alpha_{v} \rho_{v} u_{v}\right)+\sum_{k=1}^{n k}\left(\alpha_{v} \rho_{v} w_{v}\right)_{k}=\Gamma^{\prime \prime \prime}+\sum_{k=1}^{n k}\left(M_{v}^{T^{\prime \prime \prime}}\right)_{k} \\
\frac{\partial}{\partial t}\left(\alpha_{l} \rho_{l}\right)+\frac{\partial}{\partial x}\left(\alpha_{l} \rho_{l} u_{l}\right)+\sum_{k=1}^{n k}\left(\alpha_{l} \rho_{l} w_{l}\right)_{k}=-\Gamma^{\prime \prime \prime}(1-\eta)-S^{\prime \prime \prime}+\sum_{k=1}^{n k}\left(M_{l}^{T^{\prime \prime \prime}}\right)_{k} \\
\frac{\partial}{\partial t}\left(\alpha_{e} \rho_{l}\right)+\frac{\partial}{\partial x}\left(\alpha_{e} \rho_{l} u_{e}\right)+\sum_{k=1}^{n k}\left(\alpha_{e} \rho_{l} w_{e}\right)_{k}=-\Gamma^{\prime \prime \prime} \eta+S^{\prime \prime \prime}+\sum_{k=1}^{n k}\left(M_{e}^{T^{\prime \prime \prime}}\right)_{k}
\end{gathered}
$$

\subsubsection{Momentum Conservation Equations in Sub-Channel Coordinates}

Momentum equations are needed only for the axial and lateral direction for each field. The axial momentum equations for vapor, liquid, and entrained droplets are presented in Equations 2.29-2.31. The additional terms, $A, A_{k}$, and $S_{k}$, are the axial cross-sectional area, gap cross-sectional area, and gap width, respectively. The axial momentum equations have an advection term dedicated to the axial direction and a second advection term that handles transverse convection of axial momentum from all existing volume gaps. All gaps that are connected to the axial momentum cell are considered for the transverse advection term in the axial momentum equation.

$$
\begin{gathered}
\frac{\partial}{\partial t}\left(\alpha_{v} \rho_{v} u_{v} A\right)+\frac{\partial}{\partial x}\left(\alpha_{v} \rho_{v} u_{v} u_{v} A\right)+\sum_{k=1}^{n k}\left(\alpha_{v} \rho_{v} u_{v} w_{v} S\right)_{k} \\
=-\alpha_{v} A \frac{\partial P}{\partial x}-\alpha_{v} \rho_{v} g A-\tau_{\mathrm{wx}, \mathrm{v}}^{\prime \prime \prime} A-\tau_{\mathrm{ix}, \mathrm{vl}}^{\prime \prime \prime} A-\tau_{\mathrm{ix}, \mathrm{ve}}^{\prime \prime \prime} A+\Gamma^{\prime \prime \prime} u A+\sum_{k=1}^{n k}\left(M_{v}^{T^{\prime \prime \prime}} A\right)_{k} \\
\frac{\partial}{\partial t}\left(\alpha_{l} \rho_{l} u_{l} A\right)+\frac{\partial}{\partial x}\left(\alpha_{l} \rho_{l} u_{l} u_{l} A\right)+\sum_{k=1}^{n k}\left(\alpha_{l} \rho_{l} u_{l} w_{l} S\right)_{k}=-\alpha_{l} A \frac{\partial P}{\partial x}-\alpha_{l} \rho_{l} g A-\tau_{\mathrm{wx}, \mathrm{l}}^{\prime \prime \prime} A+\tau_{\mathrm{ix}, \mathrm{vl}}^{\prime \prime \prime} A \\
-\Gamma^{\prime \prime \prime}(1-\eta) u A-S^{\prime \prime \prime} u A+\sum_{k=1}^{n k}\left(M_{l}^{T^{\prime \prime \prime}} A\right)_{k}
\end{gathered}
$$




$$
\begin{array}{r}
\frac{\partial}{\partial t}\left(\alpha_{e} \rho_{l} u_{e} A\right)+\frac{\partial}{\partial x}\left(\alpha_{e} \rho_{l} u_{e} u_{e} A\right)+\sum_{k=1}^{n k}\left(\alpha_{e} \rho_{l} u_{e} w_{e} S\right)_{k}=-\alpha_{e} A \frac{\partial P}{\partial x}-\alpha_{e} \rho_{l} g A-\tau_{\mathrm{wx}, \mathrm{e}}^{\prime \prime \prime} A+\tau_{\mathrm{ix}, \mathrm{ve}}^{\prime \prime \prime} A \\
-\Gamma^{\prime \prime \prime} \eta u A+S^{\prime \prime \prime} u A+\sum_{k=1}^{n k}\left(M_{e}^{T^{\prime \prime \prime}} A\right)_{k}
\end{array}
$$

The transverse momentum equations, which are solved for transverse momentum cells (to be later discussed in Section 2.3), have advection terms for the axial direction and for the direction of the gap that is being modeled. The transverse directions that are orthogonal to the gap direction are not considered in the subchannel approach. Further, gaps cannot convect transverse momentum from and to one another because they don't know of each other's existence. Instead, transverse momentum simply "disappears" when it leaves a gap and goes into a channel. The lateral advection term in the equations will be convecting zero transverse momentum into the gap if fluid velocity is going into the gap, whereas it will convect the gap momentum out of the gap if fluid velocity is going out of the gap.

Lateral momentum equations are shown in Equations 2.32-2.34. Axial velocity can still convect lateral momentum in and out of the transverse momentum cells because gap cells are aware of other gaps above and below them. The sub-channel momentum equations are further described after the CTF mesh and finite-differenced conservation equations are described in Section 2.3.

$$
\begin{gathered}
\frac{\partial}{\partial t}\left(\alpha_{v} \rho_{v} w_{v}\right)+\frac{\partial}{\partial z}\left(\alpha_{v} \rho_{v} w_{v} w_{v}\right)+\frac{\partial}{\partial x}\left(\alpha_{v} \rho_{v} w_{v} u_{v}\right)=-\alpha_{v} \frac{\partial P}{\partial z}-\tau_{\mathrm{wx}, \mathrm{v}}^{\prime \prime \prime}-\tau_{\mathrm{ix}, \mathrm{vl}}^{\prime \prime \prime}-\tau_{\mathrm{ix}, \mathrm{ve}}^{\prime \prime \prime}+\Gamma^{\prime \prime \prime} w \\
\frac{\partial}{\partial t}\left(\alpha_{l} \rho_{l} w_{l}\right)+\frac{\partial}{\partial z}\left(\alpha_{l} \rho_{l} w_{l} w_{l}\right)+\frac{\partial}{\partial x}\left(\alpha_{l} \rho_{l} w_{l} u_{l}\right)=-\alpha_{l} \frac{\partial P}{\partial z}-\tau_{\mathrm{wx}, 1}^{\prime \prime \prime}+\tau_{\mathrm{ix}, \mathrm{vl}}^{\prime \prime \prime}-\Gamma^{\prime \prime \prime}(1-\eta) w-S^{\prime \prime \prime} w \\
\frac{\partial}{\partial t}\left(\alpha_{e} \rho_{l} w_{e}\right)+\frac{\partial}{\partial z}\left(\alpha_{e} \rho_{l} w_{e} w_{e}\right)+\frac{\partial}{\partial x}\left(\alpha_{e} \rho_{l} w_{e} u_{e}\right)=-\alpha_{e} \frac{\partial P}{\partial z}-\tau_{\mathrm{wx}, 1}^{\prime \prime \prime}+\tau_{\mathrm{ix}, \mathrm{ve}}^{\prime \prime \prime}-\Gamma^{\prime \prime \prime} \eta w+S^{\prime \prime \prime} w
\end{gathered}
$$

\subsubsection{Energy Conservation Equations in Sub-Channel Coordinates}

The vapor and liquid energy conservation equations are given in Equations 2.35 and 2.36.

$$
\frac{\partial}{\partial t}\left(\alpha_{v} \rho_{v} h_{v}\right)+\frac{\partial}{\partial x}\left(\alpha_{v} \rho_{v} h_{v} u_{v}\right)+\sum_{k=1}^{n k}\left(\alpha_{v} \rho_{v} h_{v} w_{v}\right)_{k}=-\sum_{k=1}^{n k} q_{v}^{T^{\prime \prime \prime}}+\Gamma^{\prime \prime \prime} h+q_{w v}^{\prime \prime \prime}+\alpha_{v} \frac{\partial P}{\partial t}
$$




$$
\begin{aligned}
& \frac{\partial}{\partial t}\left(\alpha_{l} \rho_{l} h_{l}\right)+\frac{\partial}{\partial x}\left(\alpha_{l} \rho_{l} h_{l} u_{l}\right)+\frac{\partial}{\partial x}\left(\alpha_{e} \rho_{l} h_{l} u_{e}\right)+\sum_{k=1}^{n k}\left(\alpha_{l} \rho_{l} h_{l} w_{l}\right)_{k}+\sum_{k=1}^{n k}\left(\alpha_{e} \rho_{l} h_{l} w_{e}\right)_{k} \\
& \quad=-\sum_{k=1}^{n k} q_{l}^{T^{\prime \prime \prime}}-\Gamma^{\prime \prime \prime} h+q_{w l}^{\prime \prime \prime}+\left(\alpha_{l}+\alpha_{e}\right) \frac{\partial P}{\partial t}
\end{aligned}
$$

\subsection{Discretized Conservation Equations and Computational Mesh}

\subsubsection{CTF Flow Volume Meshing Approach}

For the aforementioned conservation equations to be of practical use, they must be applied in some way to the modeling geometry. This is achieved by generating a mesh (grid) of volumes and then setting up the collection of mass, momentum, and energy equations for each field in each of the mesh cells. Actually, two meshes are utilized which are staggered from one another. One mesh - further called the scalar mesh is used to define the scalar variables (e.g. $\alpha, P, h$, and fluid properties). The second mesh - further called the momentum mesh — is used to defined the fluid velocity field. The choice for a staggered mesh comes down to numerical stability and accuracy issues which are discussed in more detail in Patankar [3].

Technically speaking, there are actually three different meshes; the momentum mesh is comprised of transverse momentum cells and axial momentum cells. This is due to the fact that we solve for both transverse and axial velocities. Figure $2.2[4]$ shows how scalar mesh cells and axial momentum mesh cells overlap. Note that the scalar mesh cell and the axial momentum mesh cell just above it have the same index identifier (in the figure and this document, they are differentiated by capital and lowercase letters, but in the actual CTF source code, the case is the same).

The scalar quantities are defined at the center of the scalar mesh cells and the velocities are defined at the center of the momentum cells. As a result of this fact and the staggered mesh approach, the axial velocities will be available at the top and bottom faces of the scalar mesh cell. In a similar fashion, the transverse velocities will be available on the side faces of the scalar mesh cells because the transverse momentum cells overlap the scalar mesh cell sides, as shown in Figure 2.3 [4]. Note that in Figure 2.3, the adjacent lowerand higher-numbered scalar mesh cells are labeled with indices $i i$ and $j j$.

The mesh generation process is handled by CTF after basic model information is provided by the user. That basic information includes the number of model sections, channels per section, scalar mesh cells per channel, and channel connection information. A section is a grouping of channels which are all of the same total length. Sections communicate with one another via mass, energy, and momentum transport, but only in the axial direction (i.e. there are no gaps between sections). The user defines the number of channels located within each section and then tells CTF how the channels communicate with channels above or below the section they reside in (or if they don't communicate with any channels, be they next to a boundary).

Inside the section, the user declares which sub-channels communicate laterally via gaps, the channel geometry information, and the number of scalar mesh cells within the channels. It is also possible to have variable scalar mesh cell lengths in a section, should it be necessary to obtain greater flow field detail in a certain model location. An example of a model mesh is presented in Figure 2.4 [5]. 


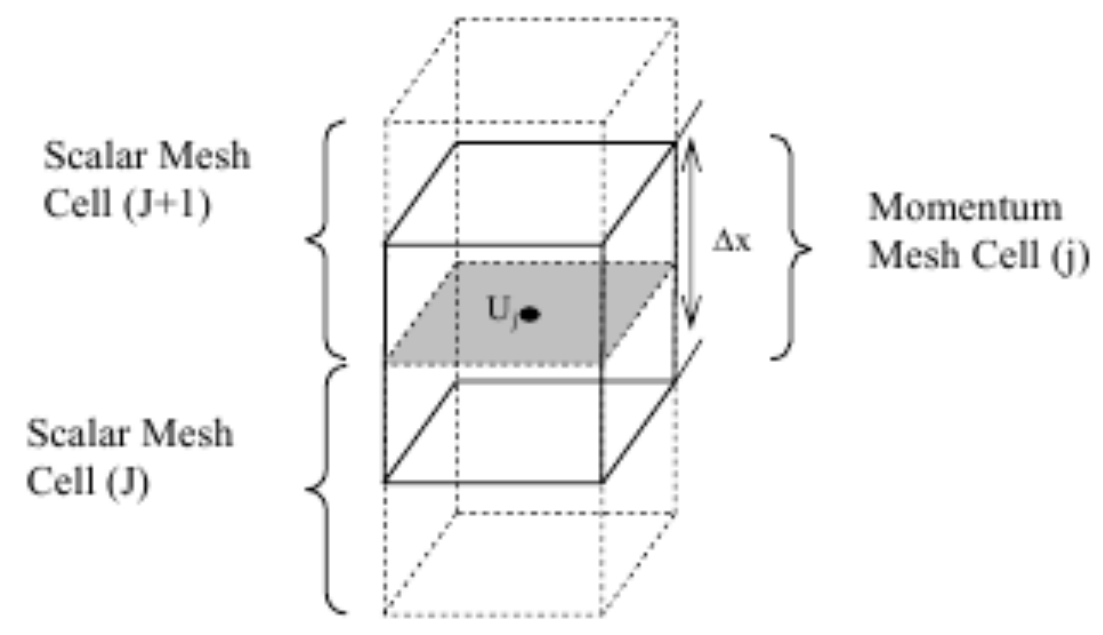

Figure 2.2: Scalar mesh cell and axial momentum mesh cell configuration

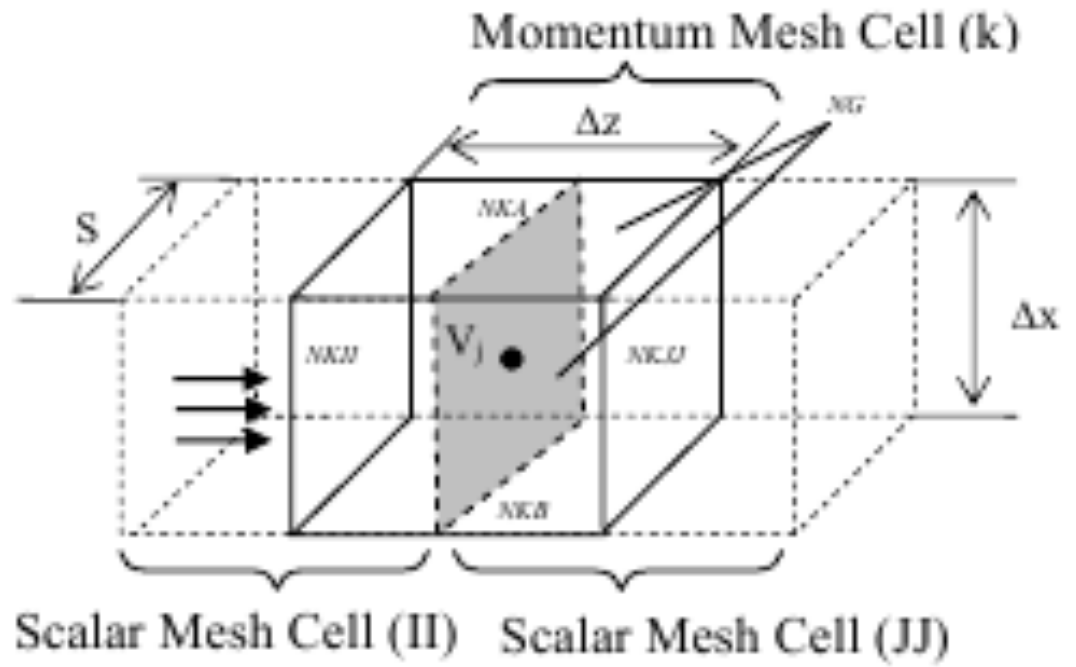

Figure 2.3: Scalar mesh cell and transverse momentum mesh cell configuration 


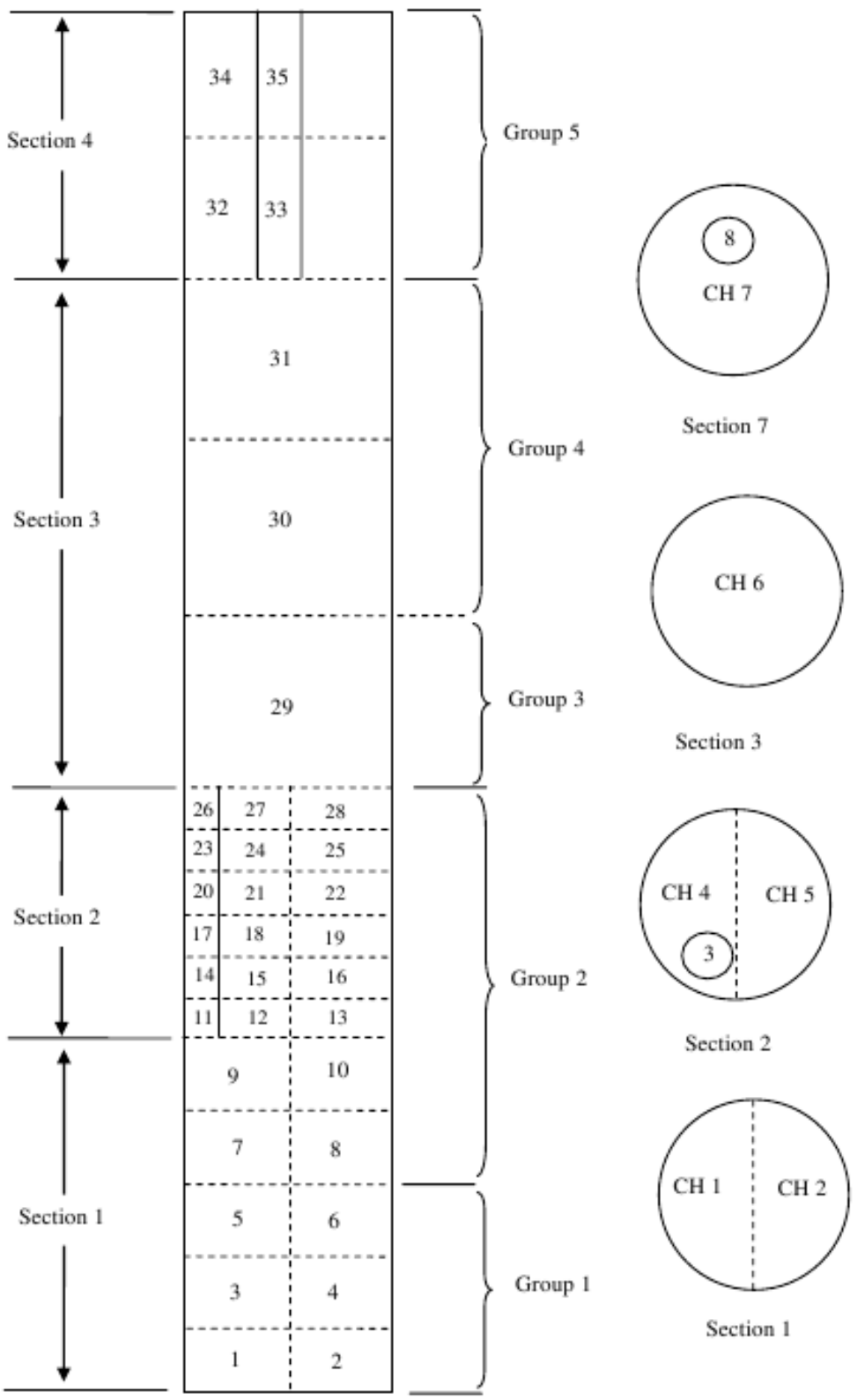

Figure 2.4: Example of a CTF mesh 
Those features are the extent of user-control over the model meshing procedure. With that information, CTF will make a structured scalar mesh grid and then connect the scalar mesh cells with axial momentum cells. A transverse momentum cell is created for each scalar cell in channels that are defined to have gaps - if no gaps are defined, no transverse momentum cells will be created.

Finite-difference forms of the conservation equations which can be solved by numerical method are then defined for the solution mesh. The discretized forms, like the previous forms, can be in Cartesian or subchannel forms, but, as will be shown, actual transverse direction is not important for the mass and energy equations. The user has the responsibility of choosing which form of the conservation equations should be used. If the Cartesian form is chosen, it is necessary for the user to enter extra information, such as the other gaps that each gap faces and which gaps convect momentum in the orthogonal direction.

\subsubsection{Finite-Difference Conservation Equations}

With the basic flow modeling equations laid out and the flow volume meshing approach understood, we turn to reforming the conservation equations in finite-difference form. The derivation begins with the mass conservation equation.

\subsubsection{Finite-Difference Mass Conservation Equation}

We start from the expanded mass conservation equations of Section 2.2.3.1. Equation 2.12 is repeated here, which is the expanded vapor mass conservation equation in Cartesian coordinates.

$$
\frac{\partial}{\partial t}\left(\alpha_{v} \rho_{v}\right)+\frac{\partial}{\partial x}\left(\alpha_{v} \rho_{v} u_{v}\right)+\frac{\partial}{\partial y}\left(\alpha_{v} \rho_{v} v_{v}\right)+\frac{\partial}{\partial z}\left(\alpha_{v} \rho_{v} w_{v}\right)=\Gamma^{\prime \prime \prime}+\sum_{k=1}^{n k}\left(M_{v}^{T^{\prime \prime \prime}}\right)_{k}
$$

First, the volumetric mass creation term is transformed into a total mass creation term by multiplying through by cell volume.

$$
\begin{aligned}
& \frac{\partial}{\partial t}\left(\alpha_{v} \rho_{v}\right) \Delta X \Delta Y \Delta Z+\frac{\partial}{\partial x}\left(\alpha_{v} \rho_{v} u_{v}\right) \Delta X \Delta Y \Delta Z+\frac{\partial}{\partial y}\left(\alpha_{v} \rho_{v} v_{v}\right) \Delta X \Delta Y \Delta Z \\
& \quad+\frac{\partial}{\partial z}\left(\alpha_{v} \rho_{v} w_{v}\right) \Delta X \Delta Y \Delta Z=\Gamma+\sum_{k=1}^{n k}\left(M_{v}^{T}\right)_{k}
\end{aligned}
$$

The partial derivatives are expanded by taking the difference in mass change over time and over the control volume spatial directions in the specified directions. 


$$
\begin{aligned}
& \frac{\left[\left(\alpha_{v} \rho_{v}\right)_{J}-\left(\alpha_{v} \rho_{v}\right)_{J}^{n}\right]}{\Delta t} \Delta X \Delta Y \Delta Z \\
& \quad+\frac{\sum_{i a=1}^{n a}\left[\left(\alpha_{v} \rho_{v}\right)^{\tilde{n}} u_{v, j} \Delta Y \Delta Z\right]_{i a}-\sum_{i b=1}^{n b}\left[\left(\alpha_{v} \rho_{v}\right)^{\tilde{n}} u_{v, j-1} \Delta Y \Delta Z\right]_{i b}}{\Delta X} \Delta X \\
& \quad+\frac{\sum_{k_{y+\Delta y}=1}^{n k_{y+\Delta y}}\left[\left(\alpha_{v} \rho_{v}\right)^{\tilde{n}} v_{v, J} \Delta Z \Delta X\right]_{k_{y+\Delta y}}-\sum_{k_{y}=1}^{n k_{y}}\left[\left(\alpha_{v} \rho_{v}\right)^{\tilde{n}} v_{v, J} \Delta Z \Delta X\right]_{k_{y}}}{\Delta Y} \Delta Y \\
& \quad+\frac{\sum_{k_{z+\Delta z}=1}^{n k_{z+\Delta z}}\left[\left(\alpha_{v} \rho_{v}\right)^{\tilde{n}} v_{v, J} \Delta Y \Delta X\right]_{k_{z+\Delta z}}-\sum_{k_{z}=1}^{n k_{z}}\left[\left(\alpha_{v} \rho_{v}\right)^{\tilde{n}} v_{v, J} \Delta Y \Delta X\right]_{k_{z}}}{\Delta Z} \Delta Z=\Gamma+\sum_{k=1}^{n k}\left(M_{v}^{T}\right)_{k}
\end{aligned}
$$

Starting with the first term on the LHS, the temporal change in mesh cell mass is represented by subtracting the old time step mass from the new time step mass. The old time step values are represented with an $n$ superscript.

The next three fractions are the advection terms in the $x$-, $y$ - and $z$-directions. The reason we see summation operators in these terms is because each side of the scalar mesh may have multiple connections to other cells. The first term subtracts all the scalar cell top face $(n a)$ flow rates from all the bottom face $(n b)$ flow rates. The positive direction for axial flow is defined moving from the cell bottom to cell top. The CTF convention is that flow moves from bottom to top, hence the given formulation.

A new superscript, $\tilde{n}$ appears in the advection terms. This superscript means that the scalar cell from which the term comes is dependent on the flow direction. For example, if in the axial advection term, $u_{v, j-1}$ is positive then $\left(\alpha_{v} \rho_{v}\right)_{J-1}$ will be used and mass will be taken from cell $J-1$ and deposited into cell $J$. However, if $u_{v, j-1}$ is negative, mass will be removed from cell $J$ and put into cell $J-1$ and $\left(\alpha_{v} \rho_{v}\right)_{J}$ will be used.

The second advection term subtracts all the $y+\Delta y$ face flow rates from the $y$ face flow rates. The $z$-direction advection term is similarly derived. In the next derivation, the actual orthogonal direction is collapsed into one term since the direction has no impact on the mass conservation equation. Furthermore, the correct geometry terms are used. Note that in this modification, the removal of the directional dependence in the advection terms, converts the equation into a form that would be arrived at using the sub-channel approach.

$$
\begin{aligned}
& \frac{\left[\left(\alpha_{v} \rho_{v}\right)_{J}-\left(\alpha_{v} \rho_{v}\right)_{J}^{n}\right]}{\Delta t} \Delta X_{J} A_{c, J}+\sum_{i a=1}^{n a}\left[\left(\alpha_{v} \rho_{v}\right)^{\tilde{n}} u_{v, j} A_{m, i a}\right]_{i a}-\sum_{i b=1}^{n b}\left[\left(\alpha_{v} \rho_{v}\right)^{\tilde{n}} u_{v, j-1} A_{m, i b}\right]_{i b} \\
& +\sum_{k=1}^{n k}\left[\left(\alpha_{v} \rho_{v}\right)^{\tilde{n}} w_{k, v} L_{k}\right]_{J} \Delta X_{J}=\Gamma+\sum_{k=1}^{n k}\left(M_{v}^{T}\right)_{k}+\text { Source }
\end{aligned}
$$

Here, the $\Delta X / \Delta X, \Delta Y / \Delta Y$, and $\Delta Z / \Delta Z$ terms in the advection terms have been cancelled. The scalar cell cross-sectional area has replaced the $\Delta Y \Delta Z$ term in the temporal term. The gap width, $L_{k}$, has been 
substituted for $\Delta Y$ in the transverse advection term; $L_{k} \Delta X_{J}$ is the cross-sectional area in the transverse direction that mass is convected through. As for the axial advection term, $\Delta Y \Delta Z$ has been replaced with the cross-sectional area of the axial momentum cell that carries mass into or out of the scalar mesh cell. The transverse velocity of the gap under consideration is more simply notated with $w_{k}$. Finally, a source term has been added on the RHS.

The liquid and entrained field mass conservation equations are similarly derived. The final results are shown in Equation 2.40 for liquid and Equation 2.41 for droplets. These equations represent the finite-differenced form of the mass conservation equations used in CTF which are used for either the Cartesian or sub-channel modeling approach.

$$
\begin{aligned}
& \frac{\left[\left(\alpha_{l} \rho_{l}\right)_{J}-\left(\alpha_{l} \rho_{l}\right)_{J}^{n}\right]}{\Delta t} \Delta X_{J} A_{c, J}+\sum_{i b=1}^{n b}\left[\left(\alpha_{l} \rho_{l}\right)^{\tilde{n}} u_{l, j-1} A_{m, i b}\right]_{i b}-\sum_{i a=1}^{n a}\left[\left(\alpha_{l} \rho_{l}\right)^{\tilde{n}} u_{l, j} A_{m, i a}\right]_{i a} \\
& +\sum_{k=1}^{n k}\left[\left(\alpha_{l} \rho_{l}\right)^{\tilde{n}} w_{k, l}\right]_{J} L_{k} \Delta X_{J}=-\Gamma(1-\eta)-S+\text { Source } \\
& \frac{\left[\left(\alpha_{e} \rho_{l}\right)_{J}-\left(\alpha_{e} \rho_{l}\right)_{J}^{n}\right]}{\Delta t} \Delta X_{J} A_{c, J}+\sum_{i b=1}^{n b}\left[\left(\alpha_{e} \rho_{l}\right)^{\tilde{n}} u_{l, j-1} A_{m, i b}\right]_{i b} \\
& -\sum_{i a=1}^{n a}\left[\left(\alpha_{e} \rho_{l}\right)^{\tilde{n}} u_{l, j} A_{m, i a}\right]_{i a}+\sum_{k=1}^{n k}\left[\left(\alpha_{e} \rho_{l}\right)^{\tilde{n}} w_{k, l}\right]_{J} L_{k} \Delta X_{J}=-\Gamma \eta-S+\text { Source }
\end{aligned}
$$

\subsubsection{Finite-Differenced Momentum Conservation Equation}

We begin the finite-differenced momentum equation derivation for the vapor field in the axial direction using Cartesian form. For this, we return to Equation 2.15, which is restated below for convenience.

$$
\begin{gathered}
\frac{\partial}{\partial t}\left(\alpha_{v} \rho_{v} u_{v}\right)+\frac{\partial}{\partial x}\left(\alpha_{v} \rho_{v} u_{v} u_{v}\right)+\frac{\partial}{\partial y}\left(\alpha_{v} \rho_{v} u_{v} v_{v}\right)+\frac{\partial}{\partial z}\left(\alpha_{v} \rho_{v} u_{v} w_{v}\right) \\
=-\alpha_{v} \frac{\partial P}{\partial x}-\alpha_{v} \rho_{v} g-\tau_{\mathrm{wx}, \mathrm{v}}^{\prime \prime \prime}-\tau_{\mathrm{ix}, \mathrm{vl}}^{\prime \prime \prime}-\tau_{\mathrm{ix}, \mathrm{ve}}^{\prime \prime \prime}+\Gamma^{\prime \prime \prime} u+\sum_{k=1}^{n k}\left(M_{v}^{T}\right)_{k}^{\prime \prime \prime}
\end{gathered}
$$

Like for the finite-differenced mass conservation equation, the cell volume is multiplied through the equation and discrete forms of the advection terms are expanded subtracting momentum outflows from inflows. The advection terms are moved to the RHS so that only the temporal term remains on the left. 


$$
\begin{aligned}
& \frac{\left[\left(\alpha_{v} \rho_{v} u_{v}\right)_{j}-\left(\alpha_{v} \rho_{v} u_{v}\right)_{j}^{n}\right]}{\Delta t} \Delta X \Delta Y \Delta Z=\left\{\sum_{i b=1}^{n b}\left[\left(\alpha_{v} \rho_{v} u_{v}\right)^{\tilde{n}} \bar{u}_{v}^{n}\right]_{i b} \Delta Y \Delta Z-\sum_{i a=1}^{n a}\left[\left(\alpha_{v} \rho_{v} u_{v}\right)^{\tilde{n}} \bar{u}_{v}^{n}\right]_{i a} \Delta Y \Delta Z\right\} \\
& +\left\{\left(\sum_{k b_{y}=1}^{n k b_{y}}\left[\left(\alpha_{v} \rho_{v} u_{v}\right)^{\tilde{n}} v_{v}^{n}\right]_{k b_{y}} \Delta X \Delta Z\right.\right. \\
& \left.+\sum_{k a_{y}=1}^{n k a_{y}}\left[\left(\alpha_{v} \rho_{v} u_{v}\right)^{\tilde{n}} v_{v}^{n}\right]_{k a_{y}} \Delta X \Delta Z\right) \\
& -\left(\sum_{k b_{y+\Delta y}=1}^{n k b_{y+\Delta y}}\left[\left(\alpha_{v} \rho_{v} u_{v}\right)^{\tilde{n}} v_{v}^{n}\right]_{\left(k b_{y}+\Delta y\right)} \Delta X \Delta Z\right. \\
& \left.\left.+\sum_{k a_{y+\Delta y}=1}^{n k a_{y+\Delta y}}\left[\left(\alpha_{v} \rho_{v} u_{v}\right)^{\tilde{n}} v_{v}^{n}\right]_{\left(k a_{y}+\Delta y\right)} \Delta X \Delta Z\right)\right\} \\
& +\left\{\left(\sum_{k b_{z}=1}^{n k b_{z}}\left[\left(\alpha_{v} \rho_{v} u_{v}\right)^{\tilde{n}} w_{v}^{n}\right]_{k b_{z}} \Delta X \Delta Y\right.\right. \\
& \left.+\sum_{k a_{z}=1}^{n k a_{z}}\left[\left(\alpha_{v} \rho_{v} u_{v}\right)^{\tilde{n}} w_{v}^{n}\right]_{k a_{z}} \Delta X \Delta Y\right) \\
& -\left(\sum_{k b_{z+\Delta z}=1}^{n k b_{z+\Delta z}}\left[\left(\alpha_{v} \rho_{v} u_{v}\right)^{\tilde{n}} w_{v}^{n}\right]_{\left(k b_{z}+\Delta z\right)} \Delta X \Delta Y\right. \\
& \left.\left.+\sum_{k a_{z+\Delta z}=1}^{n k a_{z+\Delta z}}\left[\left(\alpha_{v} \rho_{v} u_{v}\right)^{\tilde{n}} w_{v}^{n}\right]_{\left(k a_{z}+\Delta z\right)} \Delta X \Delta Y\right)\right\} \\
& -\left(\overline{\alpha_{v} \rho_{v}}\right)_{j}^{n} g \Delta X \Delta Y \Delta Z-\left(P_{J+1}-P_{J}\right) \bar{\alpha}_{v, j}^{n} \Delta Y \Delta Z-\tau_{w, v, j} \\
& -\tau_{i, v l, j}-\tau_{i, v e, j}+\Gamma u^{n}+\text { Source }_{v, j}+\sum_{k b=1}^{n k b}\left(M_{v}^{T}\right)_{k}+\sum_{k a=1}^{n k a}\left(M_{v}^{T}\right)_{k}
\end{aligned}
$$

The $y$ - and $z$-direction advection terms have four terms. Due to the staggered mesh approach, gaps can bring momentum into the axial momentum cell on each face from the scalar cell above or below. In the equation, the terms, $k_{y}$ and $k_{y+\Delta y}$, denote the side faces of the mesh cell in the $y$-direction. Similarly, the $k_{z}$ and $k_{z+\Delta z}$ terms denote the side faces of the mesh cell in the $z$-direction.

Note that in the advection terms, there are some terms with bars over them. These denote that the value is a calculated one. For example, in the $x$-direction advection term, the convecting velocity term has a bar over it because the velocity is not defined at the axial momentum cell faces, where it is needed. Instead, the velocities of axial momentum cell $j$ and $j-1$ must be averaged (for the lower face velocity). This is not necessary for the side faces since velocity is defined on the faces. For the pressure term, however, it is necessary to average scalar cells $J$ and $J+1$ void fractions to get the value for momentum cell $j$. 
The shear terms actually contain an implicit portion. The wall shear is defined as a coefficient times mass flow rate and the interfacial shear is defined as a coefficient times velocity.

$$
\tau_{w, x}=k_{w, x} \dot{m} \Delta X
$$

$$
\tau_{i, x}=k_{i, x} u \Delta X
$$

The wall drag model is further described in Chapter 4 and the interfacial drag model is further described in Chapter 5. It was necessary to present Equations 2.43 and 2.44 for the upcoming numerical solution discussion.

One final substitution is made in the vapor axial momentum conservation equation - the cell geometry terms (e.g. $\Delta X, \Delta Y$, and $\Delta Z$ ) are replaced with the appropriate CTF geometry terms. 


$$
\begin{aligned}
& \frac{\left[\left(\alpha_{v} \rho_{v} u_{v}\right)_{j}-\left(\alpha_{v} \rho_{v} u_{v}\right)_{j}^{n}\right]}{\Delta t} \Delta X_{j} A_{m, j}=\left\{\sum_{i b=1}^{n b}\left[\left(\alpha_{v} \rho_{v} u_{v}\right)^{\tilde{n}} \bar{u}_{v, J}^{n}\right]_{i b} A_{c, J}-\sum_{i a=1}^{n a}\left[\left(\alpha_{v} \rho_{v} u_{v}\right)^{\tilde{n}} \bar{u}_{v, J+1}^{n}\right]_{i a} A_{c, J+1}\right\} \\
& +\left\{\left(\sum_{k b_{y}=1}^{n k b_{y}}\left[\left(\alpha_{v} \rho_{v} u_{v}\right)^{\tilde{n}} v_{v}^{n}\right]_{k b_{y}} \frac{\Delta X_{J}}{2} L_{k b_{y}}\right.\right. \\
& \left.+\sum_{k a_{y}=1}^{n k a_{y}}\left[\left(\alpha_{v} \rho_{v} u_{v}\right)^{\tilde{n}} v_{v}^{n}\right]_{k a_{y}} \frac{\Delta X_{J+1}}{2} L_{k a_{y}}\right) \\
& -\left(\sum_{k b_{y+\Delta y}=1}^{n k b_{(y+\Delta y)}}\left[\left(\alpha_{v} \rho_{v} u_{v}\right)^{\tilde{n}} v_{v}^{n}\right]_{\left(k b_{y+\Delta y}\right)} \frac{\Delta X_{J}}{2} L_{k b_{y+\Delta y}}\right. \\
& \left.\left.+\sum_{k a_{(y+\Delta y)}=1}^{n k a_{y+\Delta y}}\left[\left(\alpha_{v} \rho_{v} u_{v}\right)^{\tilde{n}} v_{v}^{n}\right]_{\left(k a_{y+\Delta y}\right)} \frac{\Delta X_{J+1}}{2} L_{k a_{y+\Delta y}}\right)\right\} \\
& +\left\{\left(\sum_{k b_{z}=1}^{n k b_{z}}\left[\left(\alpha_{v} \rho_{v} u_{v}\right)^{\tilde{n}} w_{v}^{n}\right]_{k b_{z}} \frac{\Delta X_{J}}{2} L_{k b_{z}}\right.\right. \\
& \left.+\sum_{k a_{z}=1}^{n k a_{z}}\left[\left(\alpha_{v} \rho_{v} u_{v}\right)^{\tilde{n}} w_{v}^{n}\right]_{k a_{z}} \frac{\Delta X_{J+1}}{2} L_{k a_{z}}\right) \\
& -\left(\sum_{k b_{z+\Delta z}=1}^{n k b_{z+\Delta z}}\left[\left(\alpha_{v} \rho_{v} u_{v}\right)^{\tilde{n}} w_{v}^{n}\right]_{\left(k b_{z+\Delta z}\right)} \frac{\Delta X_{J}}{2} L_{k b_{z+\Delta z}}\right. \\
& \left.\left.+\sum_{k a_{z+\Delta z}=1}^{n k a_{z+\Delta z}}\left[\left(\alpha_{v} \rho_{v} u_{v}\right)^{\tilde{n}} w_{v}^{n}\right]_{\left(k a_{z+\Delta z}\right)} \frac{\Delta X_{J+1}}{2} L_{k a_{z+\Delta z}}\right)\right\} \\
& -\left(\overline{\alpha_{v} \rho_{v}}\right)_{j}^{n} g \Delta X_{j} A_{m, j}-\left(P_{J+1}-P_{J}\right) \bar{\alpha}_{v, j}^{n} A_{m, j}-\tau_{w, v, j}-\tau_{i, v l, j} \\
& -\tau_{i, v e, j}+\Gamma u^{n}+\text { Source }_{v, j}+\sum_{k b=1}^{n k b}\left(M_{v}^{T}\right)_{k}+\sum_{k a=1}^{n k a}\left(M_{v}^{T}\right)_{k}
\end{aligned}
$$

Recall that $L$ is the gap width of the gap under consideration. Some of the $\Delta X$ terms are divided by 2 ; because of the staggered mesh approach, gaps that cause transverse momentum advection into the axial momentum cell only do so though half of the side face cross-sectional area.

The axial liquid and entrained momentum equations are similarly derived and are presented in Equations 2.46 and 2.47 . 


$$
\begin{aligned}
& \frac{\left[\left(\alpha_{l} \rho_{l} u_{l}\right)_{j}-\left(\alpha_{l} \rho_{l} u_{l}\right)_{j}^{n}\right]}{\Delta t} \Delta X_{j} A_{m, j}=\left\{\sum_{i b=1}^{n b}\left[\left(\alpha_{l} \rho_{l} u_{l}\right)^{\tilde{n}} \bar{u}_{l}^{n}\right]_{i b} A_{c, J}-\sum_{i a=1}^{n a}\left[\left(\alpha_{l} \rho_{l} u_{l}\right)^{\tilde{n}} \bar{u}_{l}^{n}\right]_{i a} A_{c, J+1}\right\} \\
& +\left\{\left(\sum_{k b_{y}=1}^{n k b_{y}}\left[\left(\alpha_{l} \rho_{l} u_{l}\right)^{\tilde{n}} v_{l}^{n}\right]_{k b_{y}} \frac{\Delta X_{J}}{2} L_{k b_{y}}\right.\right. \\
& \left.+\sum_{k a_{y}=1}^{n k a_{y}}\left[\left(\alpha_{l} \rho_{l} u_{l}\right)^{\tilde{n}} v_{l}^{n}\right]_{k a_{y}} \frac{\Delta X_{J+1}}{2} L_{k a_{y}}\right) \\
& -\left(\sum_{k b_{y+\Delta y}=1}^{n k b_{y+\Delta y}}\left[\left(\alpha_{l} \rho_{l} u_{l}\right)^{\tilde{n}} v_{l}^{n}\right]_{\left(k b_{y+\Delta y}\right)} \frac{\Delta X_{J}}{2} L_{k b_{y+\Delta y}}\right. \\
& \left.\left.+\sum_{k a_{y+\Delta y}=1}^{n k a_{y+\Delta y}}\left[\left(\alpha_{l} \rho_{l} u_{l}\right)^{\tilde{n}} v_{l}^{n}\right]_{\left(k a_{y+\Delta y}\right)} \frac{\Delta X_{J+1}}{2} L_{k a y+\Delta y}\right)\right\} \\
& +\left\{\left(\sum_{k b_{z}=1}^{n k b_{z}}\left[\left(\alpha_{l} \rho_{l} u_{l}\right)^{\tilde{n}} w_{l}^{n}\right]_{k b_{z}} \frac{\Delta X_{J}}{2} L_{k b_{z}}\right.\right. \\
& \left.+\sum_{k a_{z}=1}^{n k a_{z}}\left[\left(\alpha_{l} \rho_{l} u_{l}\right)^{\tilde{n}} w_{l}^{n}\right]_{k a_{z}} \frac{\Delta X_{J+1}}{2} L_{k a_{z}}\right) \\
& -\left(\sum_{k b_{z+\Delta z}=1}^{n k b_{z+\Delta z}}\left[\left(\alpha_{l} \rho_{l} u_{l}\right)^{\tilde{n}} w_{l}^{n}\right]_{k b_{z+\Delta z}} \frac{\Delta X_{J}}{2} L_{k b_{z+\Delta z}}\right. \\
& \left.\left.+\sum_{k a_{z+\Delta z}=1}^{n k a_{z+\Delta z}}\left[\left(\alpha_{l} \rho_{l} u_{l}\right)^{\tilde{n}} w_{l}^{n}\right]_{k a_{z+\Delta z}} \frac{\Delta X_{J+1}}{2} L_{k a_{z+\Delta z}}\right\}\right) \\
& -\left(\overline{\alpha_{l} \rho_{l}}\right)_{j}^{n} g \Delta X_{j} A_{m, j}-\left(P_{J+1}-P_{J}\right) \bar{\alpha}_{l, j}^{n} A_{m, j}-\tau_{w, l, j}+\tau_{i, v l, j} \\
& -\Gamma(1-\eta) u^{n}+\text { Source }_{l, j}+\sum_{k b=1}^{n k b}\left(M_{l}^{T}\right)_{k}+\sum_{k a=1}^{n k a}\left(M_{l}^{T}\right)_{k}
\end{aligned}
$$




$$
\begin{aligned}
& \frac{\left[\left(\alpha_{e} \rho_{l} u_{e}\right)_{j}-\left(\alpha_{e} \rho_{l} u_{e}\right)_{j}^{n}\right]}{\Delta t} \Delta X_{j} A_{m, j}=\left\{\sum_{i b=1}^{n b}\left[\left(\alpha_{e} \rho_{l} u_{e}\right)^{\tilde{n}} \bar{u}_{e}^{n}\right]_{i b} A_{c, J}-\sum_{i a=1}^{n a}\left[\left(\alpha_{e} \rho_{l} u_{e}\right)^{\tilde{n}} \bar{u}_{e}^{n}\right]_{i a} A_{c, J+1}\right\} \\
& +\left\{\left(\sum_{k b_{y}=1}^{n k b_{y}}\left[\left(\alpha_{e} \rho_{l} u_{e}\right)^{\tilde{n}} v_{e}^{n}\right]_{k b_{y}} \frac{\Delta X_{J}}{2} L_{k b_{y}}\right.\right. \\
& \left.+\sum_{k a_{y}=1}^{n k a_{y}}\left[\left(\alpha_{e} \rho_{l} u_{e}\right)^{\tilde{n}} v_{e}^{n}\right]_{k a_{y}} \frac{\Delta X_{J+1}}{2} L_{k a_{y}}\right) \\
& -\left(\sum_{k b_{y+\Delta y}=1}^{n k b_{y+\Delta y}}\left[\left(\alpha_{e} \rho_{l} u_{e}\right)^{\tilde{n}} v_{e}^{n}\right]_{\left(k b_{y+\Delta y}\right)} \frac{\Delta X_{J}}{2} L_{k b_{y+\Delta y}}\right. \\
& \left.\left.+\sum_{k a_{y+\Delta y}=1}^{n k a_{y+\Delta y}}\left[\left(\alpha_{e} \rho_{l} u_{e}\right)^{\tilde{n}} v_{e}^{n}\right]_{\left(k a_{y+\Delta y}\right)} \frac{\Delta X_{J+1}}{2} L_{k a_{y+\Delta y}}\right)\right\} \\
& +\left\{\left(\sum_{k b_{z}=1}^{n k b_{z}}\left[\left(\alpha_{e} \rho_{l} u_{e}\right)^{\tilde{n}} w_{e}^{n}\right]_{k b_{z}} \frac{\Delta X_{J}}{2} L_{k b_{z}}\right.\right. \\
& \left.+\sum_{k a_{z}=1}^{n k a_{z}}\left[\left(\alpha_{e} \rho_{l} u_{e}\right)^{\tilde{n}} w_{e}^{n}\right]_{k a_{z}} \frac{\Delta X_{J+1}}{2} L_{k a_{z}}\right) \\
& -\left(\sum_{k b_{z+\Delta z}=1}^{n k b_{z+\Delta z}}\left[\left(\alpha_{e} \rho_{l} u_{e}\right)^{\tilde{n}} w_{e}^{n}\right]_{\left(k b_{z+\Delta z}\right)} \frac{\Delta X_{J}}{2} L_{k b_{z+\Delta z}}\right. \\
& \left.\left.+\sum_{k a_{z+\Delta z}=1}^{n k a_{z+\Delta z}}\left[\left(\alpha_{e} \rho_{l} u_{e}\right)^{\tilde{n}} w_{e}^{n}\right]_{\left(k a_{z+\Delta z}\right)} \frac{\Delta X_{J+1}}{2} L_{k a_{z+\Delta z}}\right)\right\} \\
& -\left(\overline{\alpha_{e} \rho_{l}}\right)_{j}^{n} g \Delta X_{j} A_{m, j}-\left(P_{J+1}-P_{J}\right) \bar{\alpha}_{e, j}^{n} A_{m, j}-\tau_{w, e, j} \\
& +\tau_{i, v e, j}-\Gamma \eta u^{n}+\text { Source }_{e, j}+\sum_{k b=1}^{n k b}\left(M_{e}^{T}\right)_{k}+\sum_{k a=1}^{n k a}\left(M_{e}^{T}\right)_{k}
\end{aligned}
$$

With the axial direction covered, we point our attention to the remaining directions. For the $y$ - and $z$ directions, the interest is on the transverse momentum cells. Actually, there is no need for a set of equations for both the $y$ - and $z$-directions because CTF only solves for one transverse momentum cell (gap) at a time. It is only important that we distinguish the transverse direction (direction going from $i i$ to $j j$ ) versus the direction orthogonal to that (see Figure 2.3). From here, we denote the transverse direction as the $y$-direction and the direction orthogonal to this as the $z$-direction.

The transverse momentum cells are in the same axial location, $J$, as adjacent scalar mesh cells and may have axial momentum cells above, $j$, and below, $j-1$. There may also be multiple connections in the transverse and orthogonal direction. Note that the axial advection term no longer requires a calculated convection velocity because the axial velocity is defined at the transverse momentum cell top and bottom faces. However, the transverse term requires that $\bar{v}$ is calculated. The vapor, liquid and entrained momentum 
equations are presented in Equations 2.48, 2.49, and 2.50.

A few new parameters are introduced, defined as follows:

- niib - the number of scalar cells below transverse momentum cell $k$ on the $i i$ side that convect transverse momentum across the bottom face

- njjb - the number of scalar cells below transverse momentum cell $k$ on the $j j$ side that convect transverse momentum across the top face

- niia - the number of scalar cells above transverse momentum cell $k$ on the $i i$ side that convect transverse momentum across the bottom face

- njja - the number of scalar cells above transverse momentum cell $k$ on the $j j$ side that convect transverse momentum across the top face 


$$
\begin{aligned}
& \frac{\left[\left(\alpha_{v} \rho_{v} v_{v}\right)_{k}-\left(\alpha_{v} \rho_{v} v_{v}\right)_{k}^{n}\right]}{\Delta t} \Delta X_{J} \Delta Y_{k} \Delta Z_{k}=\left\{\left(\sum_{i i b=1}^{n i i b}\left[\left(\alpha_{v} \rho_{v} v_{v}\right)^{\tilde{n}} u_{v}^{n}\right]_{i i b} \frac{A_{m, i i, j-1}}{2}\right.\right. \\
& \left.+\sum_{j j b=1}^{n j j b}\left[\left(\alpha_{v} \rho_{v} v_{v}\right)^{\tilde{n}} u_{v}^{n}\right]_{j j b} \frac{A_{m, j j, j-1}}{2}\right) \\
& -\left(\sum_{i i a=1}^{n i i a}\left[\left(\alpha_{v} \rho_{v} v_{v}\right)^{\tilde{n}} u_{v}^{n}\right]_{i i a} \frac{A_{m, i i, j}}{2}\right. \\
& \left.\left.+\sum_{j j a=1}^{n j j a}\left[\left(\alpha_{v} \rho_{v} v_{v}\right)^{\tilde{n}} u_{v}^{n}\right]_{j j a} \frac{A_{m, j j, j}}{2}\right)\right\} \\
& +\left\{\sum_{k_{y}=1}^{n k_{y}}\left[\left(\alpha_{v} \rho_{v} v_{v}\right)^{\tilde{n}} \bar{v}_{v}^{n}\right]_{k_{y}} \Delta X_{J} L_{k_{y}}\right. \\
& \left.-\sum_{k_{y+\Delta y}=1}^{n k_{y+\Delta y}}\left[\left(\alpha_{v} \rho_{v} v_{v}\right)^{\tilde{n}} \bar{v}_{v}^{n}\right]_{k_{y+\Delta y}} \Delta X_{J} L_{k_{y+\Delta y}}\right\} \\
& +\left\{\left(\sum_{k i i_{z}=1}^{n k i i_{z}}\left[\left(\alpha_{v} \rho_{v} v_{v}\right)^{\tilde{n}} w_{v}^{n}\right]_{k i i_{z}} \Delta X_{J} \frac{L_{k i i_{z}}}{2}\right.\right. \\
& \left.+\sum_{k j j_{z}=1}^{n k j j_{z}}\left[\left(\alpha_{v} \rho_{v} v_{v}\right)^{\tilde{n}} w_{v}^{n}\right]_{k j j_{z}} \Delta X_{J} \frac{L_{k j j_{z}}}{2}\right) \\
& -\left(\sum_{k i i_{z+\Delta z}=1}^{n k i i_{z+\Delta z}}\left[\left(\alpha_{v} \rho_{v} v_{v}\right)^{\tilde{n}} w_{v}^{n}\right]_{k_{z+\Delta z}} \Delta X_{J} \frac{L_{k i i_{z+\Delta z}}}{2}\right. \\
& \left.\left.+\sum_{k j j_{z+\Delta z}=1}^{n k j j_{z+\Delta z}}\left[\left(\alpha_{v} \rho_{v} v_{v}\right)^{\tilde{n}} w_{v}^{n}\right]_{k_{z+\Delta z}} \Delta X_{J} \frac{L_{k j j_{z+\Delta z}}}{2}\right)\right\} \\
& -\left(P_{i i}-P_{j j}\right) \bar{\alpha}_{v, k, J}^{n} \Delta X_{J} \Delta Z_{k}-\tau_{w, v, J} \\
& -\tau_{i, v l, J}-\tau_{i, v e, J}+\Gamma v^{n}+\text { Source }_{v, j}
\end{aligned}
$$


$\frac{\left[\left(\alpha_{l} \rho_{l} v_{l}\right)_{k}-\left(\alpha_{l} \rho_{l} v_{l}\right)_{k}^{n}\right]}{\Delta t} \Delta X_{J} \Delta Y_{k} \Delta Z_{k}=\left\{\left(\sum_{i i b=1}^{n i i b}\left[\left(\alpha_{l} \rho_{l} v_{l}\right)^{\tilde{n}} u_{l}^{n}\right]_{i i b} \frac{A_{m, i i, j-1}}{2}\right.\right.$

$$
\begin{aligned}
& \left.+\sum_{j j b=1}^{n j j b}\left[\left(\alpha_{l} \rho_{l} v_{l}\right)^{\tilde{n}} u_{l}^{n}\right]_{j j b} \frac{A_{m, j j, j-1}}{2}\right) \\
& \left.-\left(\sum_{i i a=1}^{n i i a}\left[\left(\alpha_{l} \rho_{l} v_{l}\right)^{\tilde{n}} u_{l}^{n}\right]_{i i a} \frac{A_{m, i i, j}}{2}+\sum_{j j a=1}^{n j j a}\left[\left(\alpha_{l} \rho_{l} v_{l}\right)^{\tilde{n}} u_{l}^{n}\right]_{j j a} \frac{A_{m, j j, j}}{2}\right)\right\} \\
& +\left\{\sum_{k_{y}=1}^{n k_{y}}\left[\left(\alpha_{l} \rho_{l} v_{l}\right)^{\tilde{n}} \bar{v}_{l}^{n}\right]_{k_{y}} \Delta X_{J} L_{k_{y}}\right. \\
& \left.-\sum_{k_{y+\Delta y}=1}^{n k_{y+\Delta y}}\left[\left(\alpha_{l} \rho_{l} v_{l}\right)^{\tilde{n}} \bar{v}_{l}^{n}\right]_{k_{y+\Delta y}} \Delta X_{J} L_{k_{y+\Delta y}}\right\} \\
& +\left\{\left(\sum_{k i i_{z}=1}^{n k i i_{z}}\left[\left(\alpha_{l} \rho_{l} v_{l}\right)^{\tilde{n}} w_{l}^{n}\right]_{k i i_{z}} \Delta X_{J} \frac{L_{k i i_{z}}}{2}\right.\right. \\
& \left.+\sum_{k j j_{z}=1}^{n k j j_{z}}\left[\left(\alpha_{l} \rho_{l} v_{l}\right)^{\tilde{n}} w_{l}^{n}\right]_{k j j_{z}} \Delta X_{J} \frac{L_{k j j_{z}}}{2}\right) \\
& -\left(\sum_{k i i_{z+\Delta z}=1}^{n k i i_{z+\Delta z}}\left[\left(\alpha_{l} \rho_{l} v_{l}\right)^{\tilde{n}} w_{l}^{n}\right]_{k i i_{z+\Delta z}} \Delta X_{J} \frac{L_{k i i_{z+\Delta z}}}{2}\right. \\
& \left.\left.+\sum_{k j j_{z+\Delta z}=1}^{n k j j_{z+\Delta z}}\left[\left(\alpha_{l} \rho_{l} v_{l}\right)^{\tilde{n}} w_{l}^{n}\right]_{k j j_{z+\Delta z}} \Delta X_{J} \frac{L_{k j j_{z+\Delta z}}}{2}\right)\right\} \\
& -\left(P_{i i}-P_{j j}\right) \bar{\alpha}_{l, k, J}^{n} \Delta X_{J} \Delta Z_{k}-\tau_{w, l, J}+\tau_{i, v l, J}-\Gamma(1-\eta) v^{n}+\text { Source }_{l, j}
\end{aligned}
$$




$$
\begin{aligned}
& \frac{\left[\left(\alpha_{e} \rho_{l} v_{e}\right)_{k}-\left(\alpha_{e} \rho_{l} v_{e}\right)_{k}^{n}\right]}{\Delta t} \Delta X_{J} \Delta Y_{k} \Delta Z_{k}=\left\{\left(\sum_{i i b=1}^{n i i b}\left[\left(\alpha_{e} \rho_{l} v_{e}\right)^{\tilde{n}} u_{e}^{n}\right]_{i i b} \frac{A_{m, i i, j-1}}{2}\right.\right. \\
& \left.+\sum_{j j b=1}^{n j j b}\left[\left(\alpha_{e} \rho_{l} v_{e}\right)^{\tilde{n}} u_{e}^{n}\right]_{j j b} \frac{A_{m, j j, j-1}}{2}\right) \\
& -\left(\sum_{i i a=1}^{\text {niia }}\left[\left(\alpha_{e} \rho_{l} v_{e}\right)^{\tilde{n}} u_{e}^{n}\right]_{i i a} \frac{A_{m, i i, j}}{2}\right. \\
& \left.\left.+\sum_{j j a=1}^{n j j a}\left[\left(\alpha_{e} \rho_{l} v_{e}\right)^{\tilde{n}} u_{e}^{n}\right]_{j j a} \frac{A_{m, j j, j}}{2}\right)\right\} \\
& +\left\{\sum_{k_{y}=1}^{n k_{y}}\left[\left(\alpha_{e} \rho_{l} v_{e}\right)^{\tilde{n}} \bar{v}_{e}^{n}\right]_{k_{y}} \Delta X_{J} L_{k_{y}}\right. \\
& \left.-\sum_{k_{y+\Delta y}=1}^{n k_{y+\Delta y}}\left[\left(\alpha_{e} \rho_{l} v_{e}\right)^{\tilde{n}} \bar{v}_{e}^{n}\right]_{k_{y+\Delta y}} \Delta X_{J} L_{k_{y+\Delta y}}\right\} \\
& +\left\{\left(\sum_{k i i_{z}=1}^{n k i i_{z}}\left[\left(\alpha_{e} \rho_{l} v_{e}\right)^{\tilde{n}} w_{e}^{n}\right]_{k i i_{z}} \Delta X_{J} \frac{L_{k i i_{z}}}{2}\right.\right. \\
& \left.+\sum_{k j j_{z}=1}^{n k j j_{z}}\left[\left(\alpha_{e} \rho_{l} v_{e}\right)^{\tilde{n}} w_{e}^{n}\right]_{k j j_{z}} \Delta X_{J} \frac{L_{k j j_{z}}}{2}\right) \\
& -\left(\sum_{k i i_{z+\Delta z}=1}^{n k i i_{z+\Delta z}}\left[\left(\alpha_{e} \rho_{l} v_{e}\right)^{\tilde{n}} w_{e}^{n}\right]_{k i i_{z+\Delta z}} \Delta X_{J} \frac{L_{k i i_{z+\Delta z}}}{2}\right. \\
& \left.\left.+\sum_{k j j_{z+\Delta z}=1}^{n k j j_{z+\Delta z}}\left[\left(\alpha_{e} \rho_{l} v_{e}\right)^{\tilde{n}} w_{e}^{n}\right]_{k j j_{z+\Delta z}} \Delta X_{J} \frac{L_{k j j_{z+\Delta z}}}{2}\right)\right\} \\
& -\left(P_{i i}-P_{j j}\right) \bar{\alpha}_{e, k, J}^{n} \Delta X_{J} \Delta Z_{k}-\tau_{w, e, J}+\tau_{i, v e, J}-\Gamma \eta v^{n}+\text { Source }_{e, j}
\end{aligned}
$$

The sub-channel form of the finite-differenced momentum equations can easily be derived from the Cartesian forms by eliminating directional dependence in the transverse directions. The axial vapor momentum equation is transformed into sub-channel form and shown in Equation 2.51. 


$$
\begin{aligned}
\frac{\left[\left(\alpha_{v} \rho_{v} u_{v}\right)_{j}-\left(\alpha_{v} \rho_{v} u_{v}\right)_{j}^{n}\right]}{\Delta t} \Delta X_{j} A_{m, j}= & \left\{\sum_{i b=1}^{n b}\left[\left(\alpha_{v} \rho_{v} u_{v}\right)^{\tilde{n}} \bar{u}_{v, J}^{n}\right]_{i b} A_{c, J}-\sum_{i a=1}^{n a}\left[\left(\alpha_{v} \rho_{v} u_{v}\right)^{\tilde{n}} \bar{u}_{v, J+1}^{n}\right]_{i a} A_{c, J+1}\right\} \\
& +\left\{\sum_{k b=1}^{n k b}\left[\left(\alpha_{v} \rho_{v} u_{v}\right)^{\tilde{n}} w_{v}^{n}\right]_{k b} \frac{\Delta X_{J}}{2} L_{k b}\right. \\
& \left.+\sum_{k a=1}^{n k a}\left[\left(\alpha_{v} \rho_{v} u_{v}\right)^{\tilde{n}} w_{v}^{n}\right]_{k a} \frac{\Delta X_{J+1}}{2} L_{k a}\right\} \\
& -\left(\overline{\alpha_{v} \rho_{v}}\right)_{j}^{n} g \Delta X_{j} A_{m, j}-\left(P_{J+1}-P_{J}\right) \bar{\alpha}_{v, j}^{n} A_{m, j}-\tau_{w, v, j}-\tau_{i, v l, j} \\
& -\tau_{i, v e, j}+\Gamma u^{n}+\text { Source }_{v, j}+\sum_{k b=1}^{n k b}\left(M_{v}^{T}\right)_{k}+\sum_{k a=1}^{n k a}\left(M_{v}^{T}\right)_{k}
\end{aligned}
$$

Again, all gap velocities are represented by $w$. Transverse velocities are summed over all the gaps that connect to axial momentum cell $j$, both from above and below. The liquid and entrained sub-channel momentum conservation equations are shown in Equations 2.52 and 2.53.

$$
\begin{aligned}
\frac{\left[\left(\alpha_{l} \rho_{l} u_{l}\right)_{j}-\left(\alpha_{l} \rho_{l} u_{l}\right)_{j}^{n}\right]}{\Delta t} \Delta X_{j} A_{m, j}= & \left\{\sum_{i b=1}^{n b}\left[\left(\alpha_{l} \rho_{l} u_{l}\right)^{\tilde{n}} \bar{u}_{l}^{n}\right]_{i b} A_{c, J}-\sum_{i a=1}^{n a}\left[\left(\alpha_{l} \rho_{l} u_{l}\right)^{\tilde{n}} \bar{u}_{l}^{n}\right]_{i a} A_{c, J+1}\right\} \\
& +\left\{\sum_{k b=1}^{n k b}\left[\left(\alpha_{l} \rho_{l} u_{l}\right)^{\tilde{n}} w_{l}^{n}\right]_{k b} \frac{\Delta X_{J}}{2} L_{k b}+\sum_{k a=1}^{n k a}\left[\left(\alpha_{l} \rho_{l} u_{l}\right)^{\tilde{n}} w_{l}^{n}\right]_{k a} \frac{\Delta X_{J+1}}{2} L_{k a}\right\} \\
& -\left(\overline{\alpha_{l} \rho_{l}}\right)_{j}^{n} g \Delta X_{j} A_{m, j}-\left(P_{J+1}-P_{J}\right) \bar{\alpha}_{l, j}^{n} A_{m, j}-\tau_{w, l, j}+\tau_{i, v l, j} \\
& -\Gamma(1-\eta) u^{n}+\text { Source }_{l, j}+\sum_{k b=1}^{n k b}\left(M_{l}^{T}\right)_{k}+\sum_{k a=1}^{n k a}\left(M_{l}^{T}\right)_{k}
\end{aligned}
$$

$$
\begin{aligned}
\frac{\left[\left(\alpha_{e} \rho_{l} u_{e}\right)_{j}-\left(\alpha_{e} \rho_{l} u_{e}\right)_{j}^{n}\right]}{\Delta t} \Delta X_{j} A_{m, j}= & \left\{\sum_{i b=1}^{n b}\left[\left(\alpha_{e} \rho_{l} u_{e}\right)^{\tilde{n}} \bar{u}_{e}^{n}\right]_{i b} A_{c, J}-\sum_{i a=1}^{n a}\left[\left(\alpha_{e} \rho_{l} u_{e}\right)^{\tilde{n}} \bar{u}_{e}^{n}\right]_{i a} A_{c, J+1}\right\} \\
& +\left\{\sum_{k b=1}^{n k b}\left[\left(\alpha_{e} \rho_{l} u_{e}\right)^{\tilde{n}} w_{e}^{n}\right]_{k b} \frac{\Delta X_{J}}{2} L_{k b}\right. \\
& \left.+\sum_{k a=1}^{n k a}\left[\left(\alpha_{e} \rho_{l} u_{e}\right)^{\tilde{n}} w_{e}^{n}\right]_{k a} \frac{\Delta X_{J+1}}{2} L_{k a}\right\} \\
& +\left(\overline{\alpha_{e} \rho_{l}}\right)_{j}^{n} g \Delta X_{j} A_{m, j}-\left(P_{J+1}-P_{J}\right) \bar{\alpha}_{e, j}^{n} A_{m, j}-\tau_{w, e, j} \\
& +\tau_{i, v e, j}-\Gamma \eta u^{n}+\text { Source }_{e, j}+\sum_{k b=1}^{n k b}\left(M_{e}^{T}\right)_{k}+\sum_{k a=1}^{n k a}\left(M_{e}^{T}\right)_{k}
\end{aligned}
$$


The lateral momentum equation in sub-channel form for vapor is shown in Equation 2.54.

$$
\begin{aligned}
\frac{\left[\left(\alpha_{v} \rho_{v} w_{v}\right)_{k}-\left(\alpha_{v} \rho_{v} w_{v}\right)_{k}^{n}\right]}{\Delta t} \Delta X_{J} L_{k} \Delta Z_{k}= & \left\{\sum_{i b=1}^{n b}\left[\left(\alpha_{v} \rho_{v} w_{v}\right)^{\tilde{n}} \bar{w}_{v}^{n}\right]_{i b} A_{c, J}-\sum_{i a=1}^{n a}\left[\left(\alpha_{v} \rho_{v} w_{v}\right)^{\tilde{n}} \bar{w}_{v}^{n}\right]_{i a} A_{c, J+1}\right\} \\
& +\left\{\left(0\left[\left[\bar{w}_{v}, 0\right]\right]-\left(\overline{\alpha_{v} \rho_{v}} w_{v}\right)_{k}^{n}\left[\left[-\bar{w}_{v}, 0\right]\right]\right) \Delta X_{J} L_{k}\right. \\
\left.-\left(-\left(\overline{\alpha_{v} \rho_{v}} w_{v}\right)_{k}^{n}\left[\left[\bar{w}_{v}, 0\right]\right]+0\left[\left[-\bar{w}_{v}, 0\right]\right]\right) \Delta X_{J} L_{k}\right\} & -\left(P_{i i}-P_{j j}\right) \bar{\alpha}_{v, k}^{n} \Delta X_{J} L_{k}-\tau_{w, v, k}-\tau_{i, v l, k}-\tau_{i, v e, k}+\Gamma w^{n}+S o u r c e_{v, k}
\end{aligned}
$$

An important change has been made to the sub-channel form of the transverse momentum equation. First, there is no orthogonal convection of transverse momentum into the transverse momentum cell - transverse momentum can only enter through axial and transverse connections. Second, the transverse momentum cell of interest, $k$, no longer knows of other transverse momentum cells that may be near it. Therefore, there is no summation over connecting gaps for the transverse advection term.

It also stands to reason that if the gap doesn't know of other gaps, it cannot convect transverse momentum in from other gaps and it cannot convect its momentum to other gaps. Moreover, the velocity at the gap faces cannot be calculated using other gap velocity information and, instead, we average the gap $k$ velocity with adjacent channel transverse velocity, which we must assume to be zero. In other words, gap $k$ face velocities are calculated to be one half of the gap $k$ center velocity.

In keeping with the CTF global coordinate system, positive transverse velocity moves from channel $i i$ to channel $j j$. A positive gap $k$ velocity will convect transverse momentum from channel $i i$ into gap $k$ and will convect transverse velocity in gap $k$ out to channel $j j$. Just like for channel $i i$ and $j j$ transverse velocities, we must assume that channel $i i$ and $j j$ transverse momentums are zero. Therefore, a positive gap $k$ velocity will convect zero transverse momentum into transverse momentum cell $k$, but will convect the gap $k$ transverse momentum out on the $j j$ side. Conversely, a negative gap $k$ velocity will convect the gap $k$ transverse momentum out on the $i i$ side, but will convect nothing in from the $j j$ side.

Inspection of the transverse advection term in Equation 2.54 reveals a new term, [[ $\left.\left.w_{v}, 0\right]\right]$. This term is simply defined as "the greater of the two" and it captures the above-described behavior for transverse advection using the sub-channel approach.

The liquid and entrained transverse momentum equations are similarly derived and are shown in Equations 2.55 and 2.56 .

$$
\begin{aligned}
\frac{\left[\left(\alpha_{l} \rho_{l} w_{l}\right)_{k}-\left(\alpha_{l} \rho_{l} w_{l}\right)_{k}^{n}\right]}{\Delta t} \Delta X_{J} L_{k} \Delta Z_{k}= & \left\{\sum_{i b=1}^{n b}\left[\left(\alpha_{l} \rho_{l} w_{l}\right)^{\tilde{n}_{1}} \bar{w}_{l}^{n}\right]_{i b} A_{c, J}-\sum_{i a=1}^{n a}\left[\left(\alpha_{l} \rho_{l} w_{l}\right)^{\tilde{n}} \bar{w}_{l}^{n}\right]_{i a} A_{c, J+1}\right\} \\
& +\left\{\left(0\left[\left[\bar{w}_{l}, 0\right]\right]-\left(\overline{\alpha_{l} \rho_{l}} w_{l}\right)_{k}^{n}\left[\left[-\bar{w}_{l}, 0\right]\right]\right) \Delta X_{J} L_{k}\right. \\
\left.-\left(-\left(\overline{\alpha_{l} \rho_{l}} w_{l}\right)_{k}^{n}\left[\left[\bar{w}_{l}, 0\right]\right]+0\left[\left[-\bar{w}_{l}, 0\right]\right]\right) \Delta X_{J} L_{k}\right\} & \\
& -\left(P_{i i}-P_{j j}\right) \bar{\alpha}_{l, k}^{n} \Delta X_{J} L_{k}-\tau_{w, l, k}+\tau_{i, v l, k}-\Gamma(1-\eta) w^{n}+S_{\text {ource }} e_{l, k}
\end{aligned}
$$




$$
\begin{aligned}
\frac{\left[\left(\alpha_{e} \rho_{l} w_{e}\right)_{k}-\left(\alpha_{e} \rho_{l} w_{e}\right)_{k}^{n}\right]}{\Delta t} \Delta X_{J} L_{k} \Delta Z_{k}= & \left\{\sum_{i b=1}^{n b}\left[\left(\alpha_{e} \rho_{l} w_{e}\right)^{\tilde{n}} \bar{w}_{e}^{n}\right]_{i b} A_{c, J}-\sum_{i a=1}^{n a}\left[\left(\alpha_{e} \rho_{l} w_{e}\right)^{\tilde{n}} \bar{w}_{e}^{n}\right]_{i a} A_{c, J+1}\right\} \\
& +\left\{\left(0\left[\left[\bar{w}_{e}, 0\right]\right]-\left(\overline{\alpha_{e} \rho_{l}} w_{e}\right)_{k}^{n}\left[\left[-\bar{w}_{e}, 0\right]\right]\right) \Delta X_{J} L_{k}\right. \\
\left.-\left(-\left(\overline{\alpha_{e} \rho_{l}} w_{e}\right)_{k}^{n}\left[\left[\bar{w}_{e}, 0\right]\right]+0\left[\left[-\bar{w}_{e}, 0\right]\right]\right) \Delta X_{J} L_{k}\right\} & \\
& -\left(P_{i i}-P_{j j}\right) \bar{\alpha}_{e, k}^{n} \Delta X_{J} L_{k}-\tau_{w, e, k}+\tau_{i, v e, k}-\Gamma \eta w^{n}+\text { Source }_{e, k}
\end{aligned}
$$

\subsubsection{Finite-Differenced Energy Conservation Equations}

We first restate the general-expanded vapor energy equation in Cartesian form.

$$
\frac{\partial}{\partial t}\left(\alpha_{v} \rho_{v} h_{v}\right)+\frac{\partial}{\partial x}\left(\alpha_{v} \rho_{v} h_{v} u_{v}\right)+\frac{\partial}{\partial y}\left(\alpha_{v} \rho_{v} h_{v} v_{v}\right)+\frac{\partial}{\partial z}\left(\alpha_{v} \rho_{v} h_{v} w_{v}\right)=+\frac{\partial}{\partial y} q_{v}^{T}+\frac{\partial}{\partial z} q_{v}^{T}+\Gamma^{\prime \prime \prime} h+q_{w v}^{\prime \prime \prime}+\alpha_{v} \frac{\partial P}{\partial t}
$$

The energy equations are solved for the scalar mesh cells. The equation is multiplied by mesh cell volume, the temporal term is expanded in time and the advection terms are expanded in their respective spatial directions.

$$
\begin{aligned}
& \frac{\left[\left(\alpha_{v} \rho_{v} h_{v}\right)_{J}-\left(\alpha_{v} \rho_{v} h_{v}\right)_{J}^{n}\right]}{\Delta t} \Delta X \Delta Y \Delta Z+\left\{\sum_{i b=1}^{n i b}\left[\left(\alpha_{v} \rho_{v} h_{v}\right)^{\tilde{n}} u_{v}\right]_{i b} \Delta Y \Delta Z-\sum_{i a=1}^{n i a}\left[\left(\alpha_{v} \rho_{v} h_{v}\right)^{\tilde{n}} u_{v}\right]_{i a} \Delta Y \Delta Z\right\} \\
& +\left\{\sum_{k_{y}=1}^{n k_{y}}\left[\left(\alpha_{v} \rho_{v} h_{v}\right)^{\tilde{n}} v_{v}\right]_{k_{y}} \Delta X \Delta Z-\sum_{k_{y+\Delta y}=1}^{n k_{y+\Delta y}}\left[\left(\alpha_{v} \rho_{v} h_{v}\right)^{\tilde{n}} v_{v}\right]_{k_{y+\Delta y}} \Delta X \Delta Z\right\} \\
& +\left\{\sum_{k_{z}=1}^{n k_{z}}\left[\left(\alpha_{v} \rho_{v} h_{v}\right)^{\tilde{n}} w_{v}\right]_{k_{z}} \Delta X \Delta Y-\sum_{k_{z+\Delta z}=1}^{n k_{z+\Delta z}}\left[\left(\alpha_{v} \rho_{v} h_{v}\right)^{\tilde{n}} w_{v}\right]_{k_{z+\Delta z}} \Delta X \Delta Y\right\}=q_{v, y}^{T} \Delta X \Delta Z \\
& \quad+q_{v, z} \Delta X \Delta Y+\Gamma h+q_{w, v}+\frac{\alpha_{v}^{n}\left(P-P^{n}\right)_{J}}{\Delta t} \Delta Y \Delta Z
\end{aligned}
$$

The appropriate CTF geometry terms are substituted in Equation 2.58. Also, just like for the mass equations, the transverse direction, be it the $y$ - or $z$-direction, is immaterial for the energy equation and so the $y$ - and $z$-advection terms are collapsed into one sum about the gaps connected to the scalar mesh cell.

$$
\begin{gathered}
\frac{\left[\left(\alpha_{v} \rho_{v} h_{v}\right)_{J}-\left(\alpha_{v} \rho_{v} h_{v}\right)_{J}^{n}\right]}{\Delta t} \Delta X_{J} A_{c, J}+\left\{\sum_{i b=1}^{n i b}\left[\left(\alpha_{v} \rho_{v} h_{v}\right)^{\tilde{n}} u_{v}\right]_{i b} A_{m, j-1}-\sum_{i a=1}^{n i a}\left[\left(\alpha_{v} \rho_{v} h_{v}\right)^{\tilde{n}} u_{v}\right]_{i a} A_{m, j}\right\} \\
+\sum_{k=1}^{n k}\left[\left(\alpha_{v} \rho_{v} h_{v}\right)^{\tilde{n}} w_{v}\right]_{k} \Delta X_{J} L_{k}=\sum_{k=1}^{n k}\left[q_{v}^{T} \Delta X_{J} L_{k}\right]_{k}+\Gamma h+q_{w, v}+\frac{\alpha_{v}^{n}\left(P-P^{n}\right)_{J}}{\Delta t} \Delta Y_{J} \Delta Z_{J}
\end{gathered}
$$


The liquid energy equation is presented in Equation 2.59.

$$
\begin{aligned}
& \frac{\left[\left(\left(\alpha_{l}+\alpha_{e}\right) \rho_{l} h_{l}\right)_{J}-\left(\left(\alpha_{l}+\alpha_{e}\right) \rho_{l} h_{l}\right)_{J}^{n}\right]}{\Delta t} \Delta X_{J} A_{c, J} \\
& +\left\{\sum_{i b=1}^{n i b}\left[\left(\alpha_{l} \rho_{l} h_{l}\right)^{\tilde{n}} u_{l}+\left(\alpha_{e} \rho_{l} h_{l}\right)^{\tilde{n}} u_{e}\right]_{i b} A_{m, j-1}-\sum_{i a=1}^{n i a}\left[\left(\alpha_{l} \rho_{l} h_{l}\right)^{\tilde{n}} u_{l}+\left(\alpha_{e} \rho_{l} h_{l}\right)^{\tilde{n}} u_{e}\right]_{i a} A_{m, j}\right\} \\
& \quad+\sum_{k=1}^{n k}\left[\left(\alpha_{l} \rho_{l} h_{l}\right)^{\tilde{n}} w_{l}+\left(\alpha_{e} \rho_{l} h_{l}\right)^{\tilde{n}} w_{e}\right]_{k} \Delta X_{J} L_{k}=\sum_{k=1}^{n k}\left[q_{l}^{T} \Delta X_{J} L_{k}\right]_{k} \\
& \quad-\Gamma h+q_{w, l}+\frac{\left(\alpha_{l}+\alpha_{e}\right)^{n}\left(P-P^{n}\right)_{J}}{\Delta t} \Delta Y_{J} \Delta Z_{J}
\end{aligned}
$$

Note that Equations 2.58 and 2.59 are applicable for both the Cartesian and sub-channel forms of the finite-differenced energy equations.

\subsection{Numerical Solution}

CTF uses a form of the Semi-Implicit Method for Pressure-Linked Equations (SIMPLE) to solve the conservation equations that have previously been defined. The steps of the SIMPLE algorithm, taken from Patankar [3], are:

1. Guess the pressure field, $p^{*}$.

2. Solve the momentum equations to obtain fluid velocities, $u^{*}, v^{*}$, and $w^{*}$.

3. Use the continuity equation to solve for the pressure field correction, $p^{\prime}$.

4. Calculate the corrected pressure field, $p$, by adding $p^{\prime}$ to $p^{*}$.

5. Calculate the corrected velocity field $-u, v$, and $w-$ using the corrected pressure field.

6. Solve remaining discretized equations that influence the flow field (i.e. energy equation)

7. Treat the corrected pressure, $p$, as the new guessed pressure, $p^{*}$ and repeat steps 1-6 until convergence is reached

Each of these steps is discussed with regards to the CTF solution process, as it differs in some respects. For Step 1, the user must provide a reference pressure to CTF. It will calculate the pressure field accounting for hydrostatic forces using this value and use that as the initial guess. CTF performs a solution of the conservation equations for each time step in the modeled transient - every time step after the first will use the previous time step calculated pressure field as a guess for the new one.

For Step 2, the transverse momentum equations are solved first and then the axial momentum equations. As for step 3, Patankar was considering an unheated case where fluid energy was constant; however, for our case, 
it is necessary to use both the continuity and energy equations to determine the pressure correction. The independent scalar cell properties and momentum cell mass flow rates are solved for using the previous time step values and the effect of the pressure correction. Step 3 forms what is known as the "inner iteration" - it requires the solution of the pressure correction equations. Since there will be one pressure correction equation for each scalar cell in the mesh, this can be a very large matrix to solve. CTF is capable of solving this matrix by direct Gaussian elimination or by using one of the iterative Krylov methods.

With the corrections to the pressure field calculated, the current-iteration pressure field is then determined in Step 4. Additionally, back-substitution is performed in order to get the current-iteration values for the other dependent variables (e.g. void and enthalpy). Since the pressure field was changed, the velocities are updated accordingly in Step 5.

For Step 6, other equations solved by CTF include the interfacial area transport equation for tracking the interfacial area of the droplet field and possibly fuel rod heat transfer and decay heat equations. Steps 1-6 form one cycle of what is known as an "outer iteration". CTF has a set of convergence criteria (see Section 2.4.5) that are checked upon the completion of Step 6. If they are not met, the current iteration is attempted again, but with a smaller timestep. This is done up to a user-specified number of times for each outer iteration. If the outer iteration converges, CTF then moves onto the next timestep, repeating Steps 1-6 and marching through time until the simulation is completed.

There are three main steps of this algorithm that will now be described in greater detail: solution of the momentum equations, solution of the mass and energy equations, and solution of the system pressure matrix. These steps are not mutually exclusive, as the momentum equations are initially solved and then later corrected after solution of the pressure matrix, and the derivation of the mass and energy equations are used for solution of the pressure matrix. However, it is sufficient for our purposes to lead the discussion in this manner while making note of these matters along the way.

\subsubsection{Linearization and Solution of the Momentum Equations}

With the momentum Equations, we are interested in solving for the field mass flow rates for the current time step. In the momentum equations, the new-time mass flow rates appear in the temporal term and the shear terms. Everywhere else where the mass flow rate is needed (e.g. advection terms), the old-time value is used.

The solution process is to solve the transverse momentum equations first and the axial momentum equations second. Whether we use the sub-channel or Cartesian form of the momentum equations, CTF will solve the liquid, droplet, and vapor transverse momentum equations simultaneously for one gap at a time.

Before covering the solution process for the momentum equations, we will work to reduce the finite-differenced transverse momentum equation in Cartesian form. We return to Equation 2.48: 


$$
\begin{aligned}
& \frac{\left[\left(\alpha_{v} \rho_{v} v_{v}\right)_{k}-\left(\alpha_{v} \rho_{v} v_{v}\right)_{k}^{n}\right]}{\Delta t} \Delta X_{J} \Delta Y_{k} \Delta Z_{k}=\left\{\left(\sum_{i i b=1}^{n i i b}\left[\left(\alpha_{v} \rho_{v} v_{v}\right)^{\tilde{n}} u_{v}^{n}\right]_{i i b} \frac{A_{m, i i, j-1}}{2}\right.\right. \\
& \left.+\sum_{j j b=1}^{n j j b}\left[\left(\alpha_{v} \rho_{v} v_{v}\right)^{\tilde{n}} u_{v}^{n}\right]_{j j b} \frac{A_{m, j j, j-1}}{2}\right) \\
& -\left(\sum_{i i a=1}^{n i i a}\left[\left(\alpha_{v} \rho_{v} v_{v}\right)^{\tilde{n}} u_{v}^{n}\right]_{i i a} \frac{A_{m, i i, j}}{2}\right. \\
& \left.\left.+\sum_{j j a=1}^{n j j a}\left[\left(\alpha_{v} \rho_{v} v_{v}\right)^{\tilde{n}} u_{v}^{n}\right]_{j j a} \frac{A_{m, j j, j}}{2}\right)\right\} \\
& +\left\{\sum_{k_{y}=1}^{n k_{y}}\left[\left(\alpha_{v} \rho_{v} v_{v}\right)^{\tilde{n}} \bar{v}_{v}^{n}\right]_{k_{y}} \Delta X_{J} L_{k_{y}}\right. \\
& \left.-\sum_{k_{y+\Delta y}=1}^{n k_{y+\Delta y}}\left[\left(\alpha_{v} \rho_{v} v_{v}\right)^{\tilde{n}} \bar{v}_{v}^{n}\right]_{k_{y+\Delta y}} \Delta X_{J} L_{k_{y+\Delta y}}\right\} \\
& +\left\{\left(\sum_{k i i_{z}=1}^{n k i i_{z}}\left[\left(\alpha_{v} \rho_{v} v_{v}\right)^{\tilde{n}} w_{v}^{n}\right]_{k i i_{z}} \Delta X_{J} \frac{L_{k i i_{z}}}{2}\right.\right. \\
& \left.+\sum_{k j j_{z}=1}^{n k j j_{z}}\left[\left(\alpha_{v} \rho_{v} v_{v}\right)^{\tilde{n}} w_{v}^{n}\right]_{k j j_{z}} \Delta X_{J} \frac{L_{k j j_{z}}}{2}\right) \\
& -\left(\sum_{k i i_{z+\Delta z}=1}^{n k i i_{z+\Delta z}}\left[\left(\alpha_{v} \rho_{v} v_{v}\right)^{\tilde{n}} w_{v}^{n}\right]_{k_{z+\Delta z}} \Delta X_{J} \frac{L_{k i i_{z+\Delta z}}}{2}\right. \\
& \left.\left.+\sum_{k j j_{z+\Delta z}=1}^{n k j j_{z+\Delta z}}\left[\left(\alpha_{v} \rho_{v} v_{v}\right)^{\tilde{n}} w_{v}^{n}\right]_{k_{z+\Delta z}} \Delta X_{J} \frac{L_{k j j_{z+\Delta z}}}{2}\right)\right\} \\
& -\left(P_{i i}-P_{j j}\right) \bar{\alpha}_{v, k, J}^{n} \Delta X_{J} \Delta Z_{k}-\tau_{w, v, J} \\
& -\tau_{i, v l, J}-\tau_{i, v e, J}+\Gamma v^{n}+\text { Source }_{v, j}
\end{aligned}
$$

Immediately, we can collapse the transverse momentum equation into a greatly-reduced form using the actual CTF source code variables:

- $u f o l d(2, k)$ - Transverse vapor momentum convected into cell $k$ by axial vapor velocity on the cell bottom

- uwgm - Transverse vapor momentum convected out of the cell by axial vapor velocity on the cell top

- $\operatorname{vwgm}(k)$ — Transverse vapor momentum convected into cell $k$ by orthogonal vapor velocity

- vvfii - Transverse vapor momentum convected into the cell by transverse vapor velocity on the $i i$ side of the cell 
- $v v f j j$ - Transverse vapor momentum convected out of the cell by transverse vapor velocity on the $j j$ side of the cell

- vgamv — Transverse vapor momentum convected into the cell by phase change

$$
\begin{aligned}
\frac{\left[\left(\alpha_{v} \rho_{v} v_{v}\right)_{k}-\left(\alpha_{v} \rho_{v} v_{v}\right)_{k}^{n}\right]}{\Delta t} \Delta X_{J} \Delta Y_{k} \Delta Z_{k}= & \{\text { ufold }(2, k)-u w g m\}+\{v v f i i-v v f j j\}+\{\operatorname{vwgm}(k)\} \\
& -\left(P_{i i}-P_{j j}\right) \bar{\alpha}_{v, k, J}^{n} \Delta X_{J} \Delta Z_{k}-\tau_{w, v, J}-\tau_{i, v l, J}-\tau_{i, v e, J}+v g a m v
\end{aligned}
$$

Note here that the source term was absorbed into one of the advection terms and no longer appears. Since we are solving for mass flow rates, we collapse the $\alpha \rho v A$ terms to $\dot{m}$ and expand the shear terms.

$$
\begin{aligned}
\frac{\left[\dot{m}_{v}-\dot{m}_{v}^{n}\right] \Delta X_{J}}{\Delta t}= & \{u f o l d(2, k)-u w g m\}+\{v v f i i-v v f j j\}+\{v w g m(k)\}-\left(P_{i i}-P_{j j}\right) \bar{\alpha}_{v, k, J}^{n} \Delta X_{J} \Delta Z_{k} \\
& -k_{w, x} \dot{m}_{v} \Delta X_{J}-k_{v l, x}\left(v_{v}-v_{l}\right) \Delta X_{J}-k_{v e, x}\left(v_{v}-v_{e}\right) \Delta X_{J}+v g a m v
\end{aligned}
$$

We convert the new-time velocities to new-time mass flow rates using the averaged, old-time scalar variables.

$$
\begin{aligned}
\frac{\left[\dot{m}_{v}-\dot{m}_{v}^{n}\right] \Delta X_{J}}{\Delta t}= & \{\text { ufold }(2, k)-\text { uwgmz }\}+\{v v f i i-v v f j j\}+\{v w g m(k)\}+\left(P_{j j}-P_{i i}\right) \bar{\alpha}_{v, k, J}^{n} \Delta X_{J} \Delta Z_{k} \\
& -k_{w, x} \dot{m}_{v} \Delta X_{J}-\left(\frac{k_{v l, x}}{\overline{\alpha_{v} \rho_{v}} \Delta X_{J} L_{k}}+\frac{k_{v e, x}}{\overline{\alpha_{v} \rho_{v}} \Delta X_{J} L_{k}}\right) \dot{m}_{v} \Delta X_{J} \\
& +\frac{k_{v l, x}}{\overline{\alpha_{l} \rho_{l}} \Delta X_{J} L_{k}} \dot{m}_{l} \Delta X_{J}+\frac{k_{v e, x}}{\overline{\alpha_{e} \rho_{l}} \Delta X_{J} L_{k}} \dot{m}_{e} \Delta X_{J}+v g a m v
\end{aligned}
$$

Note that we reverse the sign of the pressure term. Next, we arrange the formula so that only the new-time mass flow rate appears on the LHS.

$$
\begin{aligned}
\dot{m}_{v}= & \dot{m}_{v}^{n}+\frac{\Delta t}{\Delta X_{J}}[u f o l d(2, k)-\text { uwgm }+ \text { vvfi }-v v f j j+\text { vwgm }(k)+v g a m v] \\
& +\frac{\Delta t}{\Delta X_{J}}\left[\bar{\alpha}_{v, k, J}^{n} \Delta X_{J} \Delta Z_{k}\right] \Delta P+\frac{\Delta t}{\Delta X_{J}}\left[-k_{w, x} \Delta X_{J}-\frac{k_{v l, x}}{\overline{\alpha_{v} \rho_{v}} \Delta X_{J} L_{k}}-\frac{k_{v e, x}}{\overline{\alpha_{v} \rho_{v}} \Delta X_{J} L_{k}}\right] \dot{m}_{v} \\
& +\frac{\Delta t}{\Delta X_{J}}\left[\frac{k_{v l, x}}{\overline{\alpha_{l} \rho_{l}} \Delta X_{J} L_{k}}\right] \dot{m}_{l}+\frac{\Delta t}{\Delta X_{J}}\left[\frac{k_{v e, x}}{\overline{\alpha_{e} \rho_{l}} \Delta X_{J} L_{k}}\right] \dot{m}_{e}
\end{aligned}
$$

One further simplification can be made by substituting $A$ for the explicit terms, $B$ for the terms that multiply by pressure drop, $C$ for terms that multiply by new-time liquid mass flow rate, $D$ for terms that multiply by 
new-time vapor mass flow rate, and $E$ for terms that multiply by new-time entrained mass flow rate. This yields:

$$
\dot{m}_{v}=A_{2}+B_{2} \Delta P+C_{2} \dot{m}_{l}+D_{2} \dot{m}_{v}+E_{2} \dot{m}_{e}
$$

The subscript is used to identify the field and "2" was used to stay consistent with the CTF source code ("1" is for liquid and " $3 "$ is for droplets). Similar to the transverse vapor momentum equation, reductions can be performed for the other fields in both directions. The form of the axial and transverse momentum equations are the same. The reduced liquid and entrained momentum equations are shown below.

$$
\begin{aligned}
& \dot{m}_{l}=A_{1}+B_{1} \Delta P+C_{1} \dot{m}_{l}+D_{1} \dot{m}_{v} \\
& \dot{m}_{e}=A_{3}+B_{3} \Delta P+D_{3} \dot{m}_{v}+E_{3} \dot{m}_{e}
\end{aligned}
$$

Once the $A-E$ terms are defined in CTF, as well as the old-time pressure drop, Equations $2.64-2.66$ can be rewritten in matrix form, as follows:

$$
\left[\begin{array}{lll}
C_{1}-1 & D_{1} & 0 \\
C_{2} & D_{2}-1 & E_{2} \\
0 & D_{3} & E_{3}-1
\end{array}\right]\left\{\begin{array}{l}
\dot{m}_{l} \\
\dot{m}_{v} \\
\dot{m}_{e}
\end{array}\right\}=\left\{\begin{array}{l}
-A_{1}-B_{1} \Delta P \\
-A_{2}-B_{2} \Delta P \\
-A_{3}-B_{3} \Delta P
\end{array}\right\}
$$

It is then a simple matter to solve Equation 2.67 by Gaussian elimination and obtain the tentative mass flow rates.

\subsubsection{Linearization and Solution of Mass and Energy Equations}

At this point, the initial pressure field was used to calculate a velocity field. However the pressure field, which was based on initial conditions or the previous time step information, may be changing and the calculated velocities may not satisfy the conservation equations of mass and energy. Therefore, in this step, we determine what adjustments, if any, will need to be made to the independent variables such that the mass and energy equations are satisfied. Then we can use the newly-calculated pressure field to correct the initial velocity field calculation.

The continuity and energy equations now form a system of equations that must be solved simultaneously such that they all equal zero, and mass and energy is conserved, as shown in Equation 2.68. 


$$
\begin{aligned}
& C_{l}\left(\alpha_{v}, \alpha_{v} h_{v},\left(1-\alpha_{v}\right) h_{l}, \alpha_{e}, P_{J}, P_{i=1 \ldots n c o n n}\right)=0 \\
& E_{v}\left(\alpha_{v}, \alpha_{v} h_{v},\left(1-\alpha_{v}\right) h_{l}, \alpha_{e}, P_{J}, P_{i=1 \ldots n c o n n}\right)=0 \\
& E_{l}\left(\alpha_{v}, \alpha_{v} h_{v},\left(1-\alpha_{v}\right) h_{l}, \alpha_{e}, P_{J}, P_{i=1 \ldots n c o n n}\right)=0 \\
& C_{e}\left(\alpha_{v}, \alpha_{v} h_{v},\left(1-\alpha_{v}\right) h_{l}, \alpha_{e}, P_{J}, P_{i=1 \ldots n c o n n}\right)=0 \\
& C_{v}\left(\alpha_{v}, \alpha_{v} h_{v},\left(1-\alpha_{v}\right) h_{l}, \alpha_{e}, P_{J}, P_{i=1 \ldots n c o n n}\right)=0
\end{aligned}
$$

Note that the equations are not only functions of the mesh cell pressure that the equations are being solved in $\left(P_{J}\right)$, but also the surrounding mesh cells that connect to the cell $\left(P_{i=1 \ldots n c o n n}\right)$. This is why we must do a simultaneous solution of the pressure across the entire mesh, which is discussed next in Section 2.4.3.

We can write Equation 2.68 more conveniently in vector notation as follows:

$$
\vec{f}(\vec{x})=\overrightarrow{0}
$$

In Equation 2.69, $\vec{f}$ is vector representing the mass and energy equations, which are functions of the independent variables, represented by the $\vec{x}$ vector. Assuming all conservation equations are satisfied, they will equal the zero vector. Equation 2.69 can be solved numerically using Newton-Raphson iteration, written in Equation 2.70.

$$
\vec{f}(\vec{x})=\vec{f}\left(\vec{x}_{0}\right)+D \vec{f}\left(\vec{x}_{0}\right)\left(\vec{x}-\overrightarrow{x_{0}}\right)
$$

$D \vec{f}\left(\vec{x}_{0}\right)$ is the rate of change in each of our functions with respect to the variables. This term is known as the Jacobian. It is multiplied by the change in the variables from one iteration step to the next and added to the functions of the previous iteration variables to get the next iteration step function values. If we expand out the Jacobian term for our case, it will be the partial derivative of each conservation equation with respect to each partial derivative. 


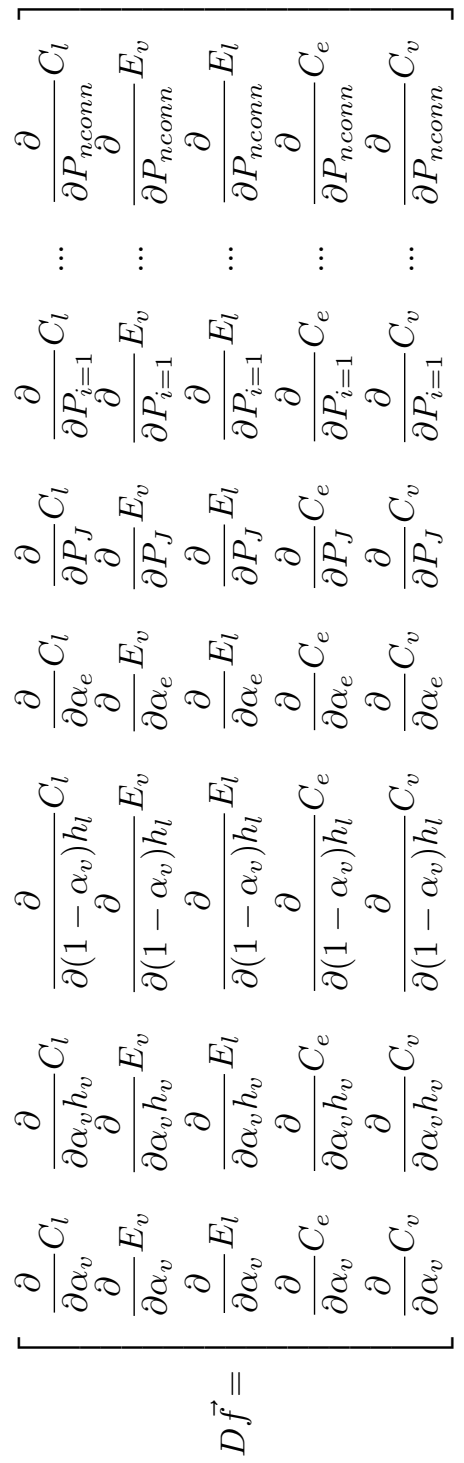


Returning to Equation 2.70, it is desirable, to satisfy the mass/energy conservation requirements, for $\vec{f}(\vec{x})$ to equal zero. Thus, our unknown becomes the amount that each variable must be changed by to achieve simultaneous solution of the system of equations. We conveniently denote the change in the variables, $\vec{x}-\overrightarrow{x_{0}}$, by $\Delta \vec{x}$ and move the terms of Equation 2.70 around to achieve the following.

$$
D \vec{f}\left(\vec{x}_{0}\right) \Delta \vec{x}=-\vec{f}\left(\vec{x}_{0}\right)
$$

The Jacobian is calculated by taking the partial derivative of each equation with respect to each variable and then substituting in the variables from the previous time step (or initial values if on the first time step). The RHS of Equation 2.72 contains the conservation equations with the previous time step values (or initial values) used for the variables. If the velocity profile that had been previously calculated by the momentum equation solution was correct (and in no need of further correction), the RHS side term would be zero. During a transient, though, it likely will not be zero and, instead, there will be some residual values.

The unknown correction term, $\Delta \vec{x}$, can be solved using Gaussian elimination if there were an equal number of unknowns and equations. However, by revisiting Equation 2.70, we can see that the matrix will not be a $n x n$ matrix because of the effect of pressure in the connecting cells and, in turn, there will be a greater number of unknowns for the current scalar cell. Recall that Equation 2.72 is setup for one scalar mesh cell. Every cell in the mesh will have its own Jacobian and its own set of residuals. This problem is remedied by forming and solving the pressure matrix for the mesh.

\subsubsection{Solution of the Pressure Matrix}

For each scalar cell in the mesh, the Jacobian matrix is reduced by Gaussian forward elimination after having been formed. The bottom of the reduced matrix will contain the pressure of the current cell and all connection cells, and will be of the form:

$$
d P_{J}=a_{5}+\sum_{i=1}^{n c o n n}\left(g_{5, i} d P_{i}\right)
$$

We see differential terms here (i.e. $d P_{J}$ ) because we are solving for the change in variables over the time step. There will be a pressure equation for each scalar mesh cell with the total number of unknown pressures equaling the total number of scalar mesh cells. This forms the pressure matrix, which may be solved by direct inversion for small computational meshes, or by Gauss-Siedel iterative technique for large meshes.

The Gauss-Siedel iterative technique involves performing direct inversion over groups of cells that are specified by the user. The solution starts in one group, performing a direct inversion over all the cells contained within that group while the cells in other groups that are attached to the current solution group retain their last iterate pressure. When direct inversion is complete, it starts again for the next group, but this time using the updated, most-recent iterate value for the previous group (if it is connected to the new solution group). This manner continues over all the groups.

This essentially reduces large, multi-dimensional problems down to one-dimensional problems having as many cells as there are solution groups. Furthermore, convergence issues that are typical of problems having 
cells with large aspect ratios (long, narrow cells) are eliminated if cells with large aspect ratios between them are placed in the same solution group.

While the direct inversion takes place for cells within the group, the pressure variation in surrounding group mesh cells are assumed to maintain their last iterate value. The process moves through each group, updating the bounding cell pressure variations with their last iterate value along the way. This has the effect of reducing large, multi-dimensional problems down to simpler

The convergence efficiency can also be greatly enhanced with the use of rebalancing. This is the process of reducing the mesh to a one-dimensional mesh and only solving pressure changes in the axial direction. Direct inversion is used, but the number of mesh cells are greatly reduced since we are only concerned with the axial direction. The solution obtained from the rebalancing approach is then used as an initial guess for each respective axial level, which starts the numerical solution closer to its actual next time step solution. The rebalancing method is optional and must be specified by the user. If the option is not used, the initial guess for linear pressure variation at each level is assumed zero.

\subsubsection{Back-substitution and Velocity Correction}

With the pressure corrections calculated for the mesh, Equation 2.73 can be solved for each scalar mesh cell to determine its new time step pressure. With the bottom of the reduced Jacobian matrix known in every scalar cell, back-substitution can be used to calculate the other new time step variables. Their forms will be:

$$
\begin{gathered}
d \alpha_{e}=a_{4}+f_{4} d P_{J}+\sum_{i=1}^{n c o n n}\left(g_{4, i} d P_{i}\right) \\
d\left[\left(1-\alpha_{v}\right) h_{l}\right]=a_{3}+e_{3} d \alpha_{e}+f_{3} d P_{J}+\sum_{i=1}^{n c o n n}\left(g_{3, i} d P_{i}\right) \\
d\left(\alpha_{v} h_{v}\right)=a_{2}+d_{2} d\left[\left(1-\alpha_{v}\right) h_{l}\right]+e_{2} d \alpha_{e}+f_{2} d P_{J}+ \\
\sum_{i=1}^{n c o n n}\left(g_{2, i} d P_{i}\right) \\
d \alpha_{v}=a_{1}+c_{1} d\left(\alpha_{v} h_{v}\right)+d_{1} d\left[\left(1-\alpha_{v}\right) h_{l}\right]+e_{1} d \alpha_{e}+ \\
f_{1} d P_{J}+\sum_{i=1}^{n c o n n}\left(g_{1, i} d P_{i}\right)
\end{gathered}
$$

The non-linear coefficients, $a_{k}$ through $g_{k}$, are assumed to remain constant over the time step, thus reducing the computational intensity of the calculation. This, however, requires that time step size is constrained to prevent the computation from going unstable, which is discussed further in Section 2.4.5.

The back-substitution would complete one iteration of the Newton-Raphson process and, at this point, it would be possible to perform further iterations; however, COBRA-TF performs a non-iterate solution in 
which the first solution obtained by the Newton-Raphson method is used as that time step's solution. By limiting the time step size, the code solution will remain stable throughout the calculation.

The velocity field is corrected for the newly calculated pressure field using the derivative of velocity with respect to pressure. It is a linear relationship which can be obtained directly from the momentum equations.

\subsubsection{Convergence Criteria}

There are two iteration loops performed in COBRA-TF. The "outer iteration" consists of setting up the continuity/energy equations over the entire mesh, reducing the Jacobian and residual arrays, and then performing back-substitution after the pressure matrix solution. The "inner iteration" consists of the solution of the pressure matrix that was created during the outer iteration. It is a separate loop that "interrupts" the flow of the outer iteration and goes over all scalar mesh cells in the model, but one that is necessary for solving the continuity/energy equations of the outer iteration.

COBRA-TF will only perform one iteration for each time step so long as convergence criteria are met. During the inner iteration, the pressure change from the previous time step is tallied for each cell and the maximum pressure change in the mesh is compared to a user-supplied convergence criterion, epso, once the inner iteration is completed. If the convergence criterion is met, the outer iteration is completed by performing back-substitution and velocity field correction. The code then moves on to the next time step.

If the convergence criterion is not met, the code will try the same time step iteration again; however, it will reduce the time step size by half in an attempt to reduce the time step pressure change. This process will be repeated up to the maximum number of iterations, oitmax, provided by the user unless convergence is achieved, in which case the code will move to the next time step.

\subsubsection{Time step Size Control}

COBRA-TF will change the time step size to improve computational efficiency. The code takes into account the Courant limit, pressure change, iteration count, void fraction change, and total error when selecting the time step size.

The Courant limit specifies time step size considering mesh size and flow velocity, as in Equation 2.75.

$$
\Delta t<\left|\frac{\Delta X}{U}\right|
$$

CTF actually uses the maximum of three different velocities to calculate the Courant limit: new time velocity, old time velocity, and an extrapolated velocity, using Equation 2.76.

$$
U_{\text {extrap }}=2 * U_{\text {new }}-U_{\text {old }}
$$

Each of these velocities is the maximum velocity in the mesh. The maximum of the three velocities and a lower limit of $1 \times 10^{-8}$ is used for calculating the Courant limit. 
The pressure change affects the time step by dividing the current time step size by the maximum pressure change. Therefore, a large pressure change will result in a small time step and, vice versa, a small pressure change will result in a large time step.

The time step due to void change is calculated as in Equation 2.77.

$$
\Delta t_{\mathrm{void}}=\Delta t_{o} * \frac{0.5}{\Delta \alpha_{v, \max }}
$$

The old time step is multiplied by 0.5 divided by the maximum void fraction change.

The number of outer and inner iterations in the previous time step affect time step size. If the number of outer iterations was greater than 5 , the ratio of 5 to the number of iterations is multiplied by time step size - the larger the number of outer iterations was, the smaller the resulting time step will be. If the number of inner iterations is larger than $70 \%$ of the maximum allowed number of inner iterations, the time step size is multiplied by the ratio of $70 \%$ of the maximum allowed number of inner iterations to number of inner iterations. If neither of these cases are true, the time step size is increased by $5 \%$.

Finally, for the total error limit, if the maximum pressure change in the mesh is greater than 0.1 for the previous time step, the new time step size is multiplied by the ratio of 0.1 to the maximum previous time step pressure change.

The minimum time step size resulting from all of these limits is used for the new time step size.

\subsubsection{Numerical Stability}

Ramps are used throughout the code to ensure numerical stability. For example, closure terms like interfacial shear and interfacial heat transfer are dependent on flow regime and, thus, require the use of different models. Ramps are used to ensure that closure terms change gradually even if a model changes from one time step to the next.

All phasic constitutive variables, such as shear and heat transfer coefficients are ramped to zero as a phase is depleted in a cell. These ramps are applied over a small range of void fractions, usually less than one percent.

Smoothing is also performed over time by using logarithmic interpolation between current time step and old time step values, as shown in Equation 2.78.

$$
y(t+\Delta t)=y(t+\Delta t)^{a} y(t)^{1-a}
$$

Here, $a$ is some parameter between 0 and 1.0 used to ramp old time step value of $y$ to the new time step calculated value in an exponential fashion. The value of $a$ in CTF is typically 0.1. 


\subsubsection{Pseudo-steady-state Option}

There exists an option in CTF to run a case as pseudo-steady-state. If this option is utilized, the solution is not true steady-state because the time term still appears in all governing equations. The solution is still solved as a transient, but at every timestep, CTF will evaluate a set of simulation convergence criteria to determine if the solution has become steady. Convergence is judged by evaluating five terms:

1. the amount of energy stored in the fluid,

2. the amount of energy stored in the solids,

3. the amount of mass stored in the system,

4. the global energy balance, and

5. the global mass balance

Each of these terms is discussed in further detail.

Transient Storage of Energy The stored energy criteria account for energy stored during the given timestep in the fluids and the solid conductors in the model. Energy storage is calculated as the change in energy in the system over the previous timestep. This is normalized by dividing through by the total power input delivered to the system from the rods. The equation for solid energy storage is as follows:

$$
E_{\text {solid storage }}=\frac{E_{\text {rod }}+E_{\text {struc }}}{Q_{\text {rod }}+Q_{\text {fluid }}} \cdot 100
$$

Energy stored in the solids is the energy change in the rods and unheated conductors over the current timestep, normalized by the rod power, $Q_{\text {rod }}$, and the direct power to the coolant, $Q_{\text {fluid }}$. The energy change in the solids are divided by the timestep size so that the units (BTU/s) matches the units of the denominator. The resulting term is multiplied by 100 to convert the units to percent.

The fluid energy storage terms is similarly calculated as follows:

$$
E_{\text {fluid storage }}=\frac{E_{\text {fluid }}}{Q_{\text {rod }}+Q_{\text {fluid }}} \cdot 100
$$

The numerator accounts for the energy change in the fluid over the previous timestep.

To expand on these terms, the energy stored in the fluid, $E_{\text {fluid }}$, is calculated as:

$$
\begin{aligned}
E_{\text {fluid }}= & \left\{\Sigma _ { \text { cell } = 1 } ^ { \text { ncells } } \left[\left|\left(\alpha_{l}+\alpha_{d}\right) h_{l} \rho_{l}-\left(\alpha_{l}^{\circ}+\alpha_{d}^{\circ}\right) h_{l}^{\circ} \rho_{l}^{\circ}\right|_{\text {cell }}+\right.\right. \\
& \left.\left.\left|\alpha_{v} h_{v} \rho_{v}-\alpha_{v}^{\circ} h_{v}^{\circ} \rho_{v}^{\circ}\right|_{\text {cell }}\right]\right\} \frac{A_{x} \Delta X}{\Delta t}
\end{aligned}
$$


In other words, it is the current-iteration energy minus the previous- iteration energy (recall that the degree symbol denotes an old-iteration value). This is done for both energy fields (recall that the droplets and liquid share an energy equation). This difference between iterations is calculated for each scalar cell and all cell storage terms are summed together to get the total system energy storage. Note that the absolute value of the liquid-energy storage is taken as well as for the vapor energy storage. It is possible that some cells may be losing energy during an iteration while others are gaining energy. Without the absolute value, it is possible for such terms to cancel each other. It is also possible for energy to pass between phases, which is why individual absolute values are taken for the two individual fields. Using the absolute value terms prevents a judge of convergence when energy is actually migrating through the system. The difference term is multiplied by the scalar cell volume and divided by the timestep size to convert the units to energy per time.

The storage terms for the solids conductors are calculated as follows:

$$
E_{\text {solid }}=\Sigma_{\text {cell }=1}^{\text {ncels }} m_{\text {cell }} C p_{\text {cell }}\left|T_{\text {cell }}-T_{\text {cell }}^{\circ}\right| \frac{1}{\Delta t}
$$

The transient storage of energy in a solid is the mass of the solid, $m_{\text {cell }}$, times its specific heat, $C p_{\text {cell }}$, times the change in temperature over the timestep. Again, an absolute value is utilized to prevent opposite energy changes in different cells from canceling one another.

Global Balance of Energy The global energy balance term accounts for all energy in and out of the system during the timestep. The equation is written:

$$
E_{\text {balance }}=\frac{Q_{\text {rod }}+Q_{\text {fluid }}+Q_{\text {inlet }}-Q_{\text {outlet }}-Q_{\text {amb }}}{Q_{\text {rod }}+Q_{\text {fluid }}+Q_{\text {inlet }}} \cdot 100
$$

The energy put into the system includes power deposited into the rods, $Q_{\text {rod, }}$ power deposited into the coolant, $Q_{\text {fluid }}$, and energy coming in at the inlet of the model, $Q_{\text {inlet }}$. The energy leaving the system includes energy leaving the outlet, $Q_{\text {outlet}}$, and energy being lost to the environment, $Q_{\text {amb }}$. This term is normalized to the sum of the energy storage terms and the energy at the inlet. The units of the heat balance term are percent.

Transient Storage of Mass The mass storage accounts for mass stored in the system during the timestep. The calculation includes all fluid cells in the mesh. This is written as:

$$
M_{\text {stor }}=\frac{M_{\text {fluid }}}{M_{\text {inlet }}} \cdot 100
$$

The stored mass is simply the amount of mass stored in all fluid cells, normalized by the mass entering the system at the inlet. The units of this term are converted to percentage. The mass stored in the coolant is simply: 


$$
M_{\text {fluid }}=\Sigma_{\text {cell }=1}^{\text {ncels }}\left[\frac{\Sigma_{k=1}^{3}\left|\alpha_{k} \rho_{k}-\alpha_{k}^{\circ} \rho_{k}^{\circ}\right|}{\Delta t}\right]
$$

It is the mass in a fluid cell at the current time (summed over all fields) minus the mass in the fluid cell at the previous iteration. An absolute value wraps each field to prevent a judge of convergence when mass is migrating between fields or throughout the system.

Global Balance of Mass Like for the energy balance, the mass balance looks at all the mass entering and leaving the system.

$$
M_{\text {balance }}=\frac{M_{\text {inlet }}-M_{\text {outlet }}}{M_{\text {inlet }}} \cdot 100
$$

The term is normalized by the amount of mass entering the system and units are, again, converted to percent.

\subsection{Boron Tracking and Precipitation Modeling}

\subsubsection{Boron Tracking Model}

The basic boron tracking algorithm is implemented in CTF such that after calculation of the fluid flow fields in each node, the boron solute flow rate is calculated at each node boundary. Based on the results of the solute flow rate, the boron mass and concentration are determined in each node.

The boron tracking model was later implemented in CTF by Ozdemir (2012) [6] with the addition of cross flow between subchannels. The importance of entrained droplet field boric acid transport was highlighted in both the Westinghouse Precipitation Modes PRT Report of Kliem et al. (2008) [7] and Tuunanen et al. (1988) [8], and his group experimental studies of Tuunanen et al. (1994) [9]. Therefore, the boron solute transient is calculated for both the continuous and entrained droplet fields (Freixa et al., 2007) [10]. The implemented boron tracking algorithm was verified using analytical and CFD solution, and subsequently validated using measured data [11] [12].

The boron tracking model performs a solution of the soluble boron conservation equation in order to track boron motion through the mesh. The advection term of the governing equation can be solved using one of two options: 1) the first-order upwind differencing scheme and, 2) the second-order modified Godunov scheme. In addition, the second-order modified Godunov scheme solves the diffusion term of the governing equation.

The following assumptions are used for the solute tracking model based on an Eularian method:

1. Liquid (solvent) properties are not altered by the presence of the solute,

2. Solute is transported only in the liquid phase and at the velocity of the liquid phase, 
3. Energy transported by the solute is negligible,

4. Inertia of the solute is negligible.

According to these assumptions, the solute field is described separately from the rest of the flow fields by a three-dimensional hyperbolic transport equation.

Based on Eulerian approach, the solution domain of the flow region is defined by computational cells, where the fluid properties can be defined as time-dependent. Beside two-phase continuity, momentum and energy equations, an additional one-dimensional field equation for the conservation of the solute is included to identify the boron concentration.

The fundamental diffusion equation, i.e. the Burgers Equation, including the physical diffusion term $D$, which makes the equation non-linear can be written as:

$$
\frac{\partial \rho_{b}}{\partial t}+\frac{v_{p} \cdot \partial \rho_{b}}{\partial x}=\frac{\partial}{\partial x}\left(D \frac{\partial \rho_{b}}{\partial x}\right)+S
$$

where $\rho_{b}$ is the spatial boron density, $v_{p}$ is the particle velocity, $D$ is the physical diffusion coefficient and $S$ is the source term. The spatial boron density, $\rho_{b}$, is defined as:

$$
\rho_{b}=\alpha_{f} \rho_{f} C_{b}=\rho_{m}(1-x) C_{b}
$$

where $\alpha_{f}$ represents the fluid void fraction; $\rho_{f}$ is the fluid density; $C_{b}$ is boron concentration; $\rho_{m}$ represents the density of the mixture and $x$ is the quality. The non-linear Burgers equation can be simplified by neglecting the diffusion coefficient. When applied to boron mass, the equation is written as follows:

$$
\frac{\partial \rho_{b}}{\partial t}+\frac{1}{A} \frac{v_{p} \cdot \partial \rho_{b} \cdot A}{\partial x}=0
$$

As discussed in the list of assumptions above, it is assumed that the particle velocity, $v_{p}$, is equal to the fluid velocity, $v_{f}$.

\subsubsection{First Order Accurate Upward Difference Scheme}

Equation 2.89 can be rewritten with respect to the boron density term $\rho_{b}$ and the speed $v$.

$$
\frac{\partial \rho_{b}}{\partial t}+v \frac{\rho_{b}}{\partial x}=0
$$

Next, Equation 2.90 can be discretized by applying a forward difference in time and a backward difference in space as: 


$$
\frac{\rho_{b, i}^{n+1}-\rho_{b, i}^{n}}{\Delta t}+\frac{v}{\Delta x}\left(\rho_{b, i}^{n}-\rho_{b, i-1}^{n}\right)=0
$$

A Taylor series expansion can be used in order to define $\rho_{b, i}^{n+1}$ and $\rho_{b, i-1}^{n}$ in terms of $\rho_{b, i}^{n}$ as:

$$
\begin{gathered}
\rho_{b, i}^{n+1}=\rho_{b, i}^{n}+\Delta t\left(\frac{\partial \rho_{b}}{\partial t}\right)_{i}^{n}+\frac{\Delta t^{2}}{2}\left(\frac{\partial^{2} \rho_{b}}{\partial t^{2}}\right)_{i}^{n}+\frac{\Delta t^{3}}{6}\left(\frac{\partial^{3} \rho b}{\partial t^{3}}\right)_{i}^{n} \\
\rho_{b, i-1}^{n}=\rho_{b, i}^{n}-\Delta x\left(\frac{\partial \rho_{b}}{\partial x}\right)_{i}^{n}+\frac{\Delta x^{2}}{2}\left(\frac{\partial^{2} \rho_{b}}{\partial x^{2}}\right)_{i}^{n}-\frac{\Delta x^{3}}{6}\left(\frac{\partial^{3} \rho_{b}}{\partial x^{3}}\right)_{i}^{n}
\end{gathered}
$$

The expression from Equation 2.92 and Equation 2.93 can be substituted in for the terms, $\frac{\rho_{b, i}^{n+1}}{\Delta t}$ and $\frac{v\left(\rho_{b, i}^{n}-\rho_{b, i-1}^{n}\right)}{\Delta x}$ in Equation 2.91. The relation between numerical scheme and the differential equation- the truncation error - $\epsilon_{t}$ is:

$$
\left[\frac{\rho_{b, i}^{n+1}-\rho_{b, i}^{n}}{\Delta t}+\frac{v}{\Delta x}\left(\rho_{b, i}^{n}-\rho_{b, i-1}^{n}\right)\right]-\left[\left(\frac{\partial \rho_{b}}{\partial t}\right)_{i}^{n}+v\left(\frac{\partial \rho_{b}}{\partial x}\right)_{i}^{n}\right]=\frac{\Delta t}{2}\left(\frac{\partial^{2} \rho_{b}}{\partial t^{2}}\right)_{i}^{n}-v \frac{\Delta x}{2}\left(\frac{\partial^{2} \rho_{b}}{\partial x^{2}}\right)_{i}^{n}+O\left(\Delta t^{2}, \Delta x^{2}\right)
$$

$$
\epsilon_{t}=\frac{\Delta t}{2}\left(\frac{\partial^{2} \rho_{b}}{\partial t^{2}}\right)_{i}^{n}-\frac{v \Delta x}{2}\left(\frac{\partial^{2} \rho_{b}}{\partial x^{2}}\right)_{i}^{n}+O\left(\Delta t^{2}, \Delta x^{2}\right)
$$

Since $\lim _{\Delta t \rightarrow 0 \Delta x \rightarrow 0}\left(\epsilon_{t}\right)=0$, the scheme can be defined as consistent. If the higher order terms, $\left(O\left(\Delta t^{2}, \Delta x^{2}\right)\right)$ are neglected, Equation 2.94 can be rewritten as:

$$
\begin{aligned}
\left(\frac{\partial \rho_{b}}{\partial t}\right)_{i}^{n}+v\left(\frac{\partial \rho_{b}}{\partial x}\right)_{i}^{n} & =-v \frac{\delta x}{2}\left(1-\frac{v \Delta t}{\Delta x}\right)\left(\frac{\partial^{2} \rho_{b}}{\partial x^{2}}\right)_{i}^{n} \\
& =-D_{n u m}\left(\frac{\partial^{2} \rho_{b}}{\partial x^{2}}\right)_{i}^{n}
\end{aligned}
$$

where:

$$
D_{n u m}=\frac{v \Delta x}{2}\left(1-\frac{v \Delta t}{\delta x}\right)
$$


From Equation 2.96 it can be concluded that, discretization of the linear convection equation, Equation 2.89 , introduces numerical diffusion $D_{\text {num }}$ (numerical viscosity) in its numerical solution, which is defined in Equation 2.97.

The numerical diffusion term, $D_{\text {num }}$ should be positive to obtain a solution damped in time. If $D_{n u m}$ becomes negative, numerical diffusion grows indefinitely in time and the solution becomes unstable. Then, for the first order upwind scheme stability, it should be had:

$$
0 \leq \frac{v \Delta t}{\Delta x} \leq 1
$$

This relation is defined as the Courant limit condition (defined in Equation 2.75), which can be given as the stability requirement with respect to the ratio between time step $(\Delta t)$ and the cell size $(\Delta x)$. The Courant limit also controls the other CTF governing equations which similarly use an upwind advection scheme. The benefit of the upwind differencing numerical scheme is that it provides a positive definite solution which increases its stability and robustness.

\subsubsection{Second Order Godunov Scheme}

Investigations conducted by Freixa et al. (2007) [10] into the accuracy of the solution methods first concluded that the upward difference scheme suffers from numerical diffusion due to numerical truncation. It was found that the second-order accurate Godunov scheme significantly reduces the numerical diffusion commonly

observed in the upward difference scheme. However, accounting for physical diffusion was still lacking in the original Godunov solution scheme. Based on finite volume method, it can be taken the volume integral of Equation 2.89 over the total volume of the cell $V$ as:

$$
\int_{V} \frac{\partial \rho_{b}}{\partial t} \partial V+\int_{V}\left(\frac{1}{A} \frac{\partial\left(\rho_{b} \cdot v_{f} \cdot A\right)}{\partial x}\right) \partial V=0
$$

Using the divergence theorem, Equation 2.99 can be re-written in a control volume $(V)$ and with a surface area $(A)$ :

$$
\int_{V} \frac{\partial \rho_{b}}{\partial t} \partial V+\int_{A} \rho_{b} \cdot v_{f} \cdot \partial A=0
$$

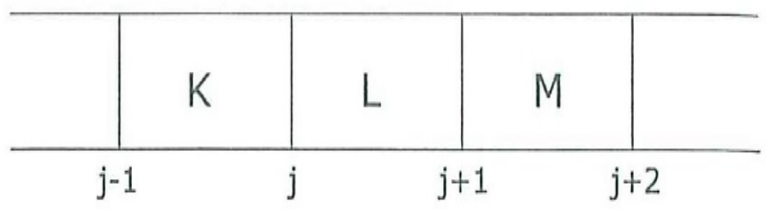

Figure 2.5: Spatial Notation Used to Demonstrate the Modified Godunov Scheme, by Ozdemir (2012) [6]. 
Based on Figure 2.5, the upward difference discretization of Equation 2.100 gives the following solution with respect to mass flux $G_{j}$ and $G_{j+1}$ :

$$
\left[\rho_{b, L}^{n+1}-\rho_{b, L}^{n}\right]+\left[\frac{\delta t}{V_{L}}\left(A_{j+1} G_{j+1}^{n}-A_{j} G_{j}^{n}\right)\right]=0
$$

The above relation gives the first order accurate Godunov scheme. Since the velocity $(v)$ is known in both old $(n)$ and new $(n+1)$ time steps, it can be linearly interpolated to generate an approximation based on time centered velocity $\left(v^{n+1 / 2)}\right)$ as:

$$
v_{f, j+1}^{n+1 / 2}=v=\frac{1}{2}\left(v_{f, j+1}^{n+1}+v_{f, j+1}^{n}\right)
$$

Then the flux terms $G_{j}$ and $G_{j+1}$ in old time domain $n$ are defined as,

$$
\begin{gathered}
G_{j+1}^{n}=\frac{1}{2}\left[v_{f, j+1}^{n+1 / 2}\left(\rho_{b, j+1}^{n, L}+\rho_{b, j+1}^{n, M}\right)+\left|v_{f, j+1}^{n+1 / 2}\left(\rho_{b, j+1}^{n, L}-\rho_{b, j+1}^{n, M}\right)\right|\right] \\
G_{j}^{n}=\frac{1}{2}\left[v_{f, j}^{n+1 / 2}\left(\rho_{b, j}^{n, L}+\rho_{b, j}^{n, M}\right)+\left|v_{f, j}^{n+1 / 2}\left(\rho_{b, j}^{n, L}-\rho_{b, j}^{n, M}\right)\right|\right]
\end{gathered}
$$

Equation 2.105 and 2.106 represent the boron density in the neighboring cells at their common interface:

$$
\begin{gathered}
\rho_{b, j+1}^{n, L}=\rho_{b, L}^{n}+\left(\frac{1}{2} \Delta x_{L}\right)\left(1-\frac{v \Delta t}{\Delta x_{L}}\right) \bar{S}_{L} \\
\rho_{b, j+1}^{n, M}=\rho_{b, M}^{n}+\left(\frac{1}{2} \Delta x_{M}\right)\left(1-\frac{v \Delta t}{\Delta x_{M}}\right) \bar{S}_{M}
\end{gathered}
$$

The cell-centered limited gradient, $\bar{S}_{L}$, is defined as:

$$
\bar{S}_{L}=\left(1+\theta_{L} \omega_{L}\right) \Phi(r, 1) S_{j+1}=\left(1+\theta_{L} \omega_{L}\right) \Phi(r, 1)\left(\frac{\rho_{b, M}^{n}-\rho_{b, L}^{n}}{\Delta x_{j+1}}\right)
$$

The cell-centered limited gradient, $\bar{S}_{L}$, provides higher order accuracy in space an time interpolation for cell edge values of $\rho_{b}$ with respect to the changes in the neighboring cells from the flux at their common interface (see Equation 2.105 and 2.106. The superbee non-linear flux limiter of Roe [13], $\Phi$, is used in order to prevent flux oscillations between adjacent cells. The effect of the flux limiter is to smooth parts of the solution near a discontinuity in the solution. The limiter switches to a first order upwind, flux-conserved advection scheme to prevent numerical instability. The flux limiter is given as,

$$
\Phi(r, 1)=\max [0, \min (2 r, 1), \min (r, 2)]
$$


where,

$$
\begin{gathered}
r=\frac{S_{j}}{S_{j+1}} \\
S_{j}=\frac{\rho_{b, L}^{n}-\rho_{b, K}^{n}}{\Delta x_{j}} \\
S_{j+1}=\frac{\rho_{b, M}^{n}-\rho_{b, L}^{n}}{\Delta x_{j+1}}
\end{gathered}
$$

The artificial compression term of Yee [14] has been introduced in order to make sure that the solution is continuous because the number of grid points tends to be relatively small and the scheme introduces spurious oscillations into the solution, where discontinuities and shocks are present. In the artificial compression term $\left(1+\theta_{L} \omega_{L}\right), \theta_{L}$, is defined as,

$$
\theta_{L}=\frac{|1-r|}{1+|r|}
$$

and the parameter $\omega_{L}$, is partially defined by the local Courant number.

$$
\omega_{L}=\min \left(C_{r, L}, 1-C_{r, L}\right)
$$

These modifications make the Godunov method second order accurate. Using the second-order accurate Godunov solution scheme and introducing a diffusion term to consider physical diffusion, a modified Godunov solution scheme was devised by Freixa. An additional diffusion term has been defined within the Godunov solution by replacing the integrated boron field in Equation 2.100

$$
\int_{V} \frac{\partial \rho_{b}}{\partial t} \partial V+\int_{A}\left(\rho_{b} \cdot v_{f}-D \frac{\partial \rho_{b}}{\partial x}\right) \cdot \partial A=0
$$

There were two physical diffusion terms $(D)$ defined based on the collision of the boron particles and turbulent phenomena which are Brownian diffusion and Eddy diffusion When a small particle is suspended in a fluid, it is subjected to the impact of the gas or liquid molecules. The instantaneous momentum given to the particle varies randomly and causes the particle to move on an erratic path known as Brownian motion. The Brownian diffusion coefficient given by Einstein [15] with the added Cunningham correlation factor [16] is

$$
D_{\text {Brownian }}=\frac{k_{b} \cdot T \cdot C_{c}}{6 \pi \cdot \rho \cdot v \cdot a}
$$

where $k_{b}$ is the Boltzmann constant, $T$ represents the temperature, $\rho$ is the fluid density, $v$ is the velocity and $a$ represents the particle radius. The Cunningham correction factor is defined as a function of $\lambda$, which represents the mean free path, and $a$ the particle radius. 


$$
C_{c}=1+\frac{\lambda}{a}\left[1.257+0.4 e^{-1.1 a / \lambda}\right]
$$

Eddy diffusion appears with turbulent phenomena. Turbulence is characterized by the motion of the fluid particles, which is irregular with respect to both direction and time. Even though the dominant velocity direction is axial, there can be regions with a direction and modulus different from those of the mean velocity. The turbulent fluid is imagined to consist of lumps of fluids, or eddies, of different sizes. Eddy diffusion is slightly different from fluid turbulent diffusion, but in this case, because of the low weight of the particles, both diffusivity will be essentially equal.

Equation 2.117 was developed by Macian-Juan and Mahaffy [17] based on the dispersion model. The suggested value for $G_{0}$ was equal to 1.35 .

$$
D_{E d d y}=G_{0} v R e^{7 / 8}
$$

While the range of values of the Brownian coefficient for boron acid particles oscillates between $10^{-6}$ and $10^{-7}$, the Eddy coefficient has a scale order of $10^{-2}$ to $10^{-3}$, depending on the fluid velocity. Consequently, Brownian diffusion is removed from the equation. Then, following the similar procedure, Equation 2.105 and, analogously Equation 2.106, is rewritten including the Eddy diffusion coefficient $D_{E}$.

$$
\rho_{b, j+1}^{n, L}=\rho_{b, L}^{n}+\left[\left(\frac{1}{2} \Delta x_{L}\right)\left(1-\frac{v \Delta t}{\Delta x_{L}}\right)-\varphi_{L}\right] \bar{S}_{L}
$$

where $\varphi$ is the limited diffusion term:

$$
\varphi_{L}=\min \left(\frac{\Delta x}{2 C_{r}}, \frac{D_{E d d y}}{v_{L}}\right)
$$

\subsubsection{Boron Precipitation Model}

The boron precipitation model was added to predict the plated out solute concentration in CTF. The solubility of boron increases with the increase of coolant temperature. Thus, it is essential to take into account the coolant temperature effect in maximum boron concentration calculations:

1. First the maximum solubility of the boric acid in $100 \mathrm{~g}$ of water (maximum mass fraction, $m_{\max }$ ) is calculated according to Kim et al.'s correlation [18] as:

$$
\frac{m_{m} a x}{100}=\psi \cdot\left(-\frac{2.68 \times 10^{8}}{2.73 .15+T_{l i q}}+6.04\right)
$$

where the conversion parameter $\psi$ is given as:

$$
\psi=3.432 \times 10^{2}\left(\lambda+1.80 \times 10^{3} \times T_{\text {liq }}\right)
$$


$\lambda=0.1795$ and $T_{\text {liq }}$ is the liquid temperature in ${ }^{\circ} \mathrm{C}$

2. Then, the maximum boron concentration is evaluated based on the amount of liquid mass $\left(m_{l i q}\right)$ both in continuous and entrained droplet liquid phases:

$$
\rho_{b, \max }=\frac{m_{b, \max }}{m_{l i q}+m_{b, \max }}
$$

From Figure 2.6, the Kim et al.'s solubility correlation is derived in a temperature range between $40-$ $160^{\circ} \mathrm{C}(313-433 \mathrm{~K})$ including the effects of buffer agents like tri-sodium phosphate (TPT), which generate particulate oxide corrosion products and increase the risk of precipitation by chemical reactions with other dissolved materials [18].

In $\mathrm{CTF}$, if the liquid temperature reaches a value outside the given range, the value at $160^{\circ} \mathrm{C}$ is used (Figure 2.7). If the new solute density goes beyond the maximum value, CTF assumes the excess is precipitated and the solute density reaches its maximum value. The maximum boron concentration profile used in CTF is given in Figure 2.7.

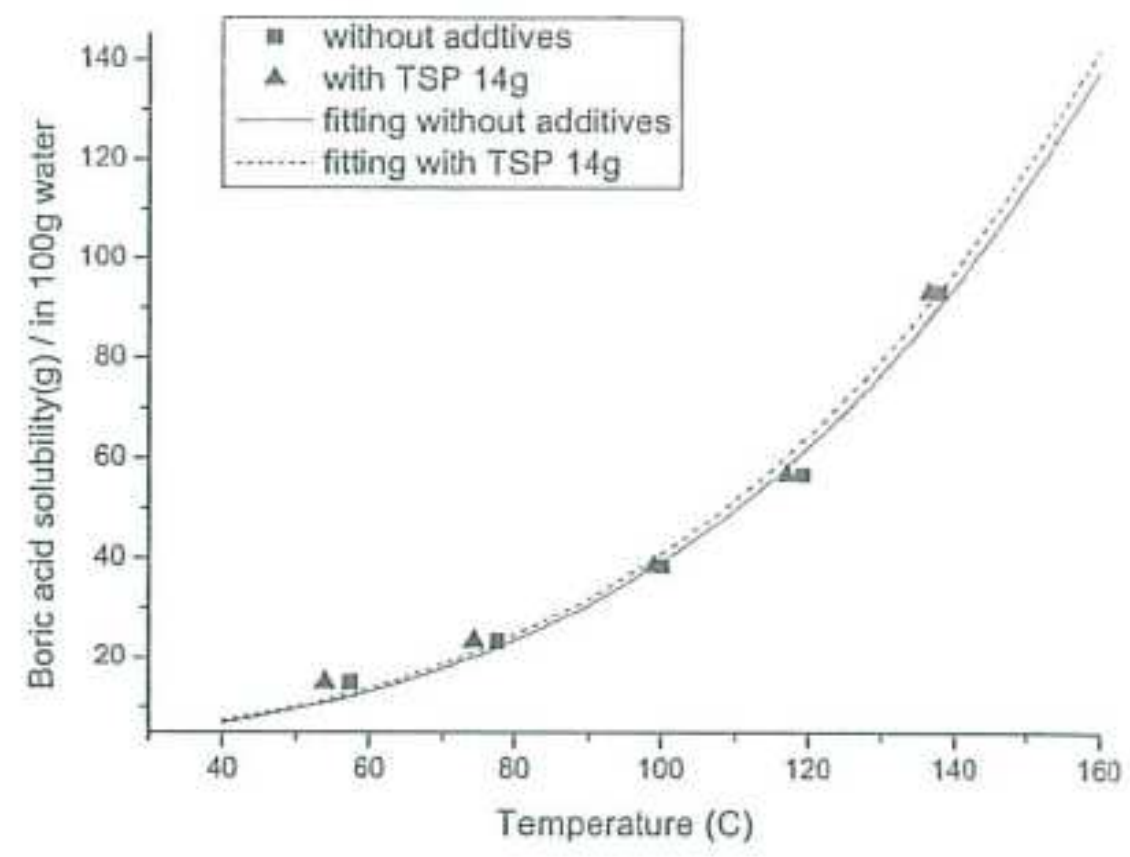

Figure 2.6: Boric Acid Solubility Correlation w.r.t. $100 g$ water, by Kim (2008) [18] 


\section{Maximum Boron Concentration}

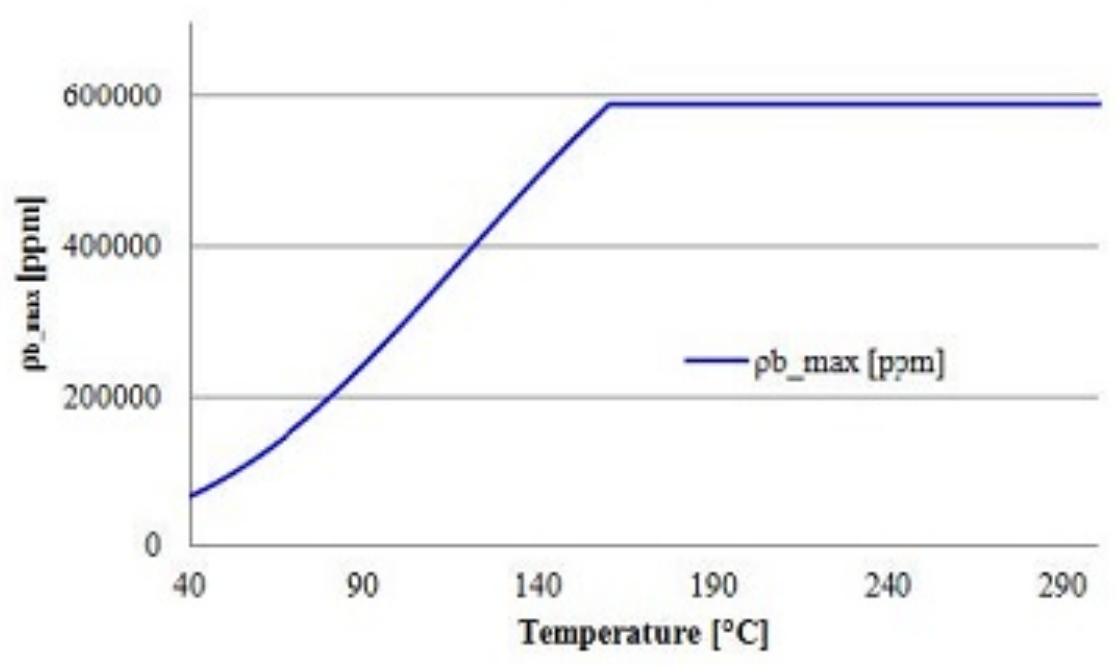

Figure 2.7: CTF Maximum Boric Acid Concentration by Kim et al.'s Correlation [18] 


\section{Flow Regime Map}

\subsection{Introduction}

The conservation equations that had been described in Chapter 2 contain terms that must be solved for prior to the conservation equation solution. These terms include the interfacial heat transfer, interfacial drag, and wall drag terms. However, these terms are dependent on the behavior of the two-phase flow, which is classified as one of several flow regimes using a flow regime map.

COBRA-TF actually contains two different types of flow regime maps: the "normal wall" map and the "hot wall" map. The normal wall map is used when the maximum wall surface temperature in the given computational mesh cell is below the critical heat flux temperature and is thus expected to be fully wetted. The hot wall map, on the other hand, is selected when the maximum wall surface temperature exceeds the critical heat flux temperature. Once the flow regime of the mesh cell is correctly identified, the appropriate models can be chosen for calculation of the closure terms.

Pertaining to the actual source code, the flow regime selection process is performed in the INTFR subroutine. Furthermore, the flow regime is defined for the momentum cells and not the scalar mesh cells. This is logical for the interfacial drag and wall drag calculations because those terms are utilized in the axial and transverse momentum equations only. However, the interfacial heat transfer term should be available in the scalar mesh cells - while it is calculated for the axial momentum cells, the interfacial heat transfer is calculated for the scalar mesh cells in the XSCHEM subroutine using linear averaging of values in adjacent axial momentum cells.

This chapter discusses the flow regime maps in COBRA-TF and the approximations used for calculating the interfacial area for each recognized flow regime.

\subsection{Normal Wall Flow Regimes}

\subsubsection{Introduction}

The normal wall flow regime map is used when the maximum wall surface temperature in the mesh cell is below the critical heat flux temperature, which is denoted by Equation 3.1. 


$$
T_{w}=\min \left(705.3^{\circ} \mathrm{F}, T_{\mathrm{CHF}}\right)
$$

The critical heat flux temperature is assumed to be well-approximated by $T_{\mathrm{CHF}}=\left(T_{\text {sat }}+75\right)^{\circ} \mathrm{F}$. The upper limit of $705.3^{\circ} \mathrm{F}$ corresponds to the critical temperature of water. If the maximum wall temperature exceeds the value given by Equation 3.1, a whole new range of flow regimes become possible since the liquid can only partially wet the wall.

The normal wall flow regime map includes the following flow regimes:

- Small-bubble

- Small-to-large bubble (Slug)

- Churn/turbulent

- Annular/mist

The map is shown graphically, including void fraction values that demarcate the transition points, in Figure 3.1. The selection logic is straightforward, but a check is first made to see if there is a large void fraction gradient (i.e. $\Delta \alpha_{v}>0.2$ ) between adjacent axial mesh cells. If yes, the minimum void fraction of the two cells is used to determine the flow regime. A diagram of the normal wall map flow regime selection logic is shown in Figure 3.2.

Each of the individual flow regimes of the normal wall map are described in the following sub-sections. The interfacial area calculation is also described for each flow regime, which is later used in Chapter 5 for calculation of the interfacial drag and interfacial heat transfer.

Returning to Figure 3.2, the void fraction check is made to ensure that the mesh cell flow regime is consistent with those around it. It is made between adjacent axial mesh cells as shown below:

$$
\Delta \alpha_{v}=\alpha_{v}(i, j+1)-\alpha_{v}(i, j)
$$

Here, $i$ is the channel index number and $j$ is the axial node index. If the void fraction difference is large (i.e. $\left.\left|\Delta \alpha_{v}\right|>0.2\right)$, a ramp is defined as:

$$
F_{\Delta \alpha}=\min \left\{\begin{array}{c}
1.0 \\
\frac{\left|\Delta \alpha_{v}\right|-0.2}{0.2}
\end{array}\right.
$$

This ramp is then used to re-define mesh cell $(i, j)$ void fraction, which is later used for calculation of interfacial quantities. The void fractions for each of the three fields become: 


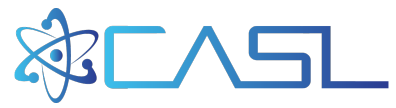

CTF Theory

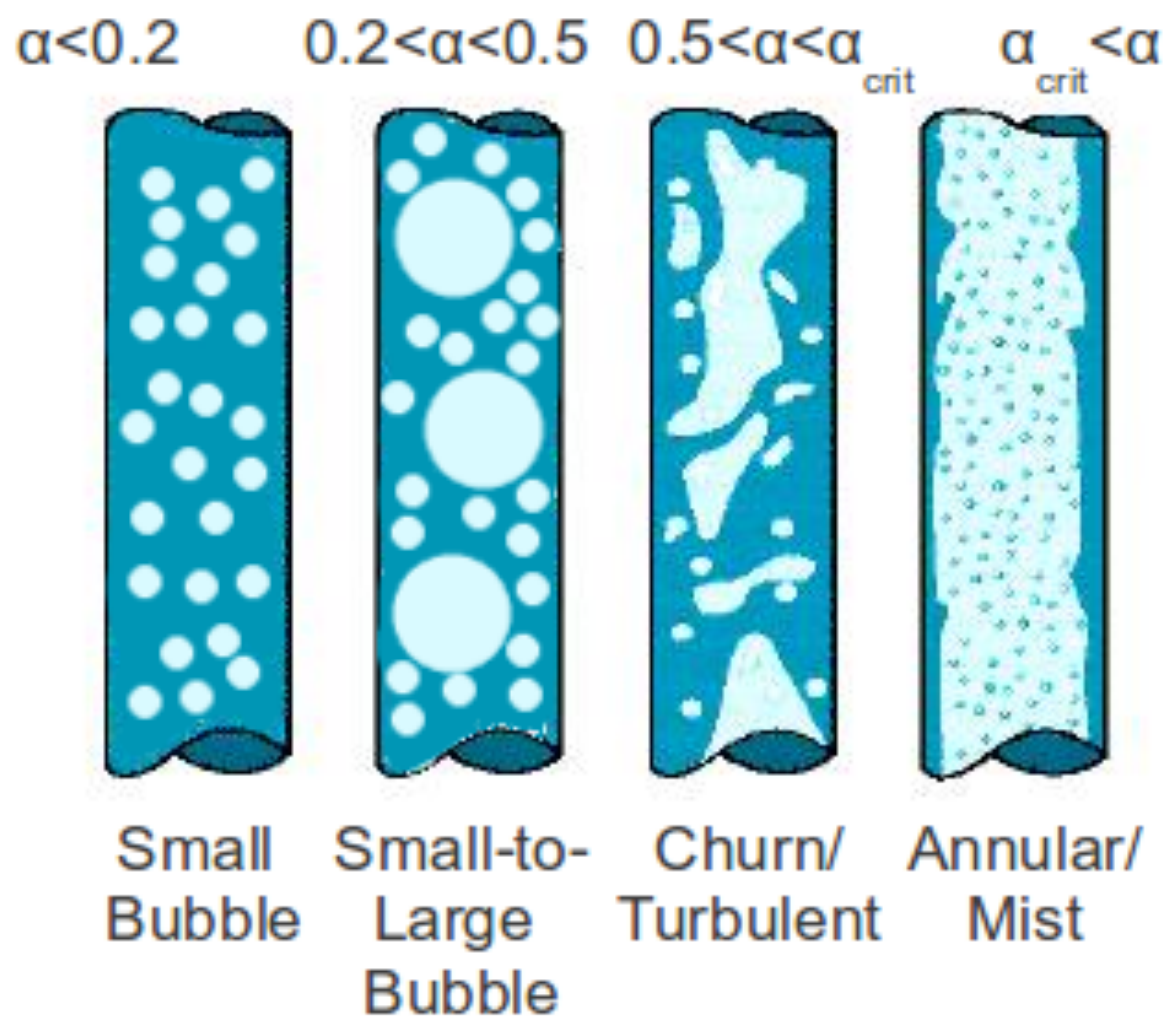

Figure 3.1: Normal wall flow regimes recognized by CTF 


\section{Normal Wall Regimes}
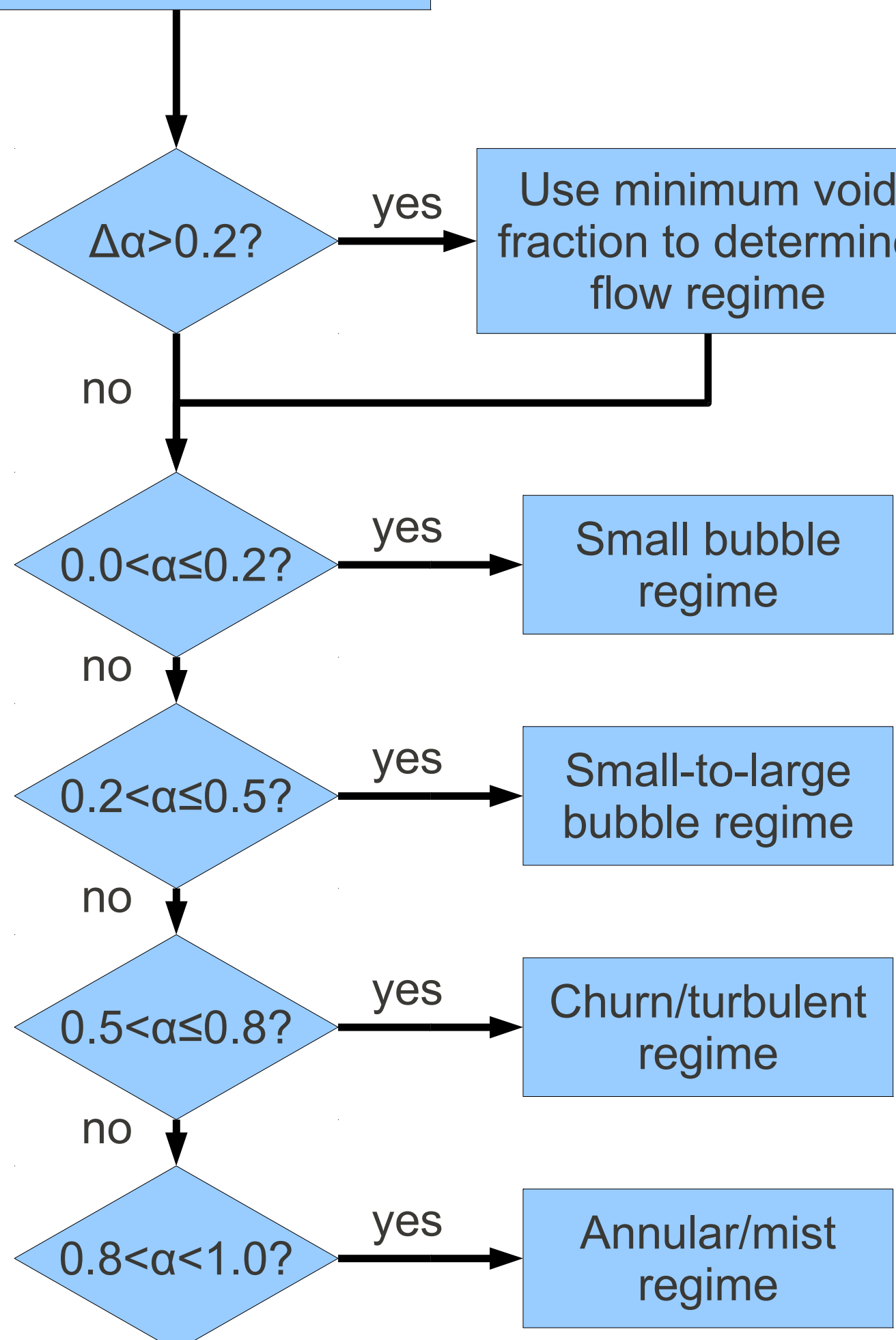


$$
\begin{aligned}
& \alpha_{v}=F_{\Delta \alpha} \alpha_{v}(i, j)+\frac{1}{2}\left(1-F_{\Delta \alpha}\right)\left[\alpha_{v}(i, j)+\alpha_{v}(i, j+1)\right] \\
& \alpha_{l}=F_{\Delta \alpha} \alpha_{l}(i, j)+\frac{1}{2}\left(1-F_{\Delta \alpha}\right)\left[\alpha_{l}(i, j)+\alpha_{l}(i, j+1)\right] \\
& \alpha_{e}=F_{\Delta \alpha} \alpha_{e}(i, j)+\frac{1}{2}\left(1-F_{\Delta \alpha}\right)\left[\alpha_{e}(i, j)+\alpha_{e}(i, j+1)\right]
\end{aligned}
$$

If the mesh cell $(i, j-1)$ is in the annular/mist or a hot wall regime, $\alpha_{v}$ and $\alpha_{l}$ are re-set for subsequent calculations to the following:

$$
\begin{aligned}
& \alpha_{v}=\max \left\{\begin{array}{c}
\alpha_{v}(i, j-1) \\
\alpha_{v}, \quad \text { from Equation } 3.4
\end{array}\right. \\
& \alpha_{l}=\min \left\{\begin{array}{cc}
\alpha_{l}(i, j-1) \\
\alpha_{l}, & \text { from Equation } 3.4
\end{array}\right.
\end{aligned}
$$

If $|\Delta \alpha|>0.2$ and $\Delta \alpha<0$, it is likely that an inverted pool exists and the void fractions used to determine the flow regime and interfacial terms in cell $(i, j)$ are:

$$
\begin{aligned}
& \alpha_{v}=F_{\Delta \alpha} \alpha_{v}(i, j+1)+\frac{1}{2}\left(1-F_{\Delta \alpha}\right)\left[\alpha_{v}(i, j)+\alpha_{v}(i, j+1)\right] \\
& \alpha_{l}=F_{\Delta \alpha} \alpha_{l}(i, j+1)+\frac{1}{2}\left(1-F_{\Delta \alpha}\right)\left[\alpha_{l}(i, j)+\alpha_{l}(i, j+1)\right] \\
& \alpha_{e}=F_{\Delta \alpha} \alpha_{e}(i, j+1)+\frac{1}{2}\left(1-F_{\Delta \alpha}\right)\left[\alpha_{e}(i, j)+\alpha_{e}(i, j+1)\right]
\end{aligned}
$$

When a large void gradient between two cells is not present, the void fractions at the momentum cell center are assumed to be equal to the average void fractions in the accompanying continuity cells:

$$
\begin{aligned}
\alpha_{v} & =\frac{1}{2}\left[\alpha_{v}(i, j)+\alpha_{v}(i, j+1)\right] \\
\alpha_{l} & =\frac{1}{2}\left[\alpha_{l}(i, j)+\alpha_{l}(i, j+1)\right] \\
\alpha_{e} & =\frac{1}{2}\left[\alpha_{e}(i, j)+\alpha_{e}(i, j+1)\right]
\end{aligned}
$$

With the appropriate void fraction determined, CTF then selects the flow regime. Each of the normal wall flow regimes are discussed in the following sub-sections. 


\subsubsection{Small Bubble Regime}

The small bubble flow regime consists of small bubbles dispersed in a continuous liquid. The regime exists for void fractions ranging from 0.0 to 0.2 . The upper range of the flow regime is the point at which small bubbles begin to coalesce and form larger bubbles. The void fraction of 0.2 was selected based on the work of several authors.

First, Griffith and Wallis [19] conducted experiments with air-water mixture in tubes with diameter up to 1.0 inch and observed that below a void fraction of 0.18 there was no indication of slug formation. Additional experiments by Griffith and Snyder [20] indicated that the void fraction where the bubbly to slug transition occurs is in the range of 0.25 to 0.30 . Mishima and Ishii [21] recommended a transition from small bubble to slug at a void fraction of 0.3. Similarly, Radovicich and Moissis [22] postulated that the maximum void fraction for bubbly flow is attained when the bubble collision frequency becomes very large and they considered this to be a void fraction of 0.30 .

The INTFR subroutine is used to determine the flow regime and flow regime-dependent closure terms. After selecting the flow regime to be small bubble, the interfacial area is calculated by assuming that the bubbles are spherical in geometry. The interfacial area is calculated in two ways: one for calculation of interfacial drag and one for interfacial heat transfer.

Interfacial Area for Interfacial Drag The radius is defined using a critical Weber number of 10.0. This calculation is performed as follows:

$$
r_{b}^{*}=\frac{0.5 W e_{\mathrm{crit}} \sigma g_{c}}{\rho_{l} \vec{u}_{v l}^{2}}+1 \cdot 10^{-5}
$$

The $\vec{u}_{v l}$ is a vector, which includes both axial and transverse components of relative velocity between the liquid and vapor phases. The bubble radius is limited so that it cannot become larger than one-half the cell hydraulic diameter or $0.02 \mathrm{ft}$, whichever is smaller. This can be written:

$$
r_{b}=\left\{\begin{array}{c}
r_{b}^{*} \\
\frac{D_{h}}{2} \\
0.02 \mathrm{ft}
\end{array}\right.
$$

The interfacial area used in the interfacial drag coefficient calculations is then estimated as:

$$
A_{i, s b}=3 \alpha_{v} A_{x} \frac{\Delta X}{r_{b}}
$$

$A_{x}$ is the momentum cell area.

Interfacial Area for Interfacial Heat Transfer If the liquid is superheated, the bubble diameter is calculated as: 


$$
\begin{aligned}
& D_{b}=\min \left(2 r_{b}, D_{b}^{*}\right), \text { where } \\
& D_{b}^{*}=2\left[\frac{3}{4 \pi} \frac{\max \left(\alpha_{v}, 0.001\right)}{N_{b}}\right]^{0.333}, \text { or } \\
& D_{b}^{*}=1.24\left[\frac{\max \left(\alpha_{v}, 0.001\right)}{N_{b}}\right]^{0.333}
\end{aligned}
$$

$N_{b}$ is the minimum bubble number desnity and is defined as:

$$
N_{b}=\frac{2000}{\max \left(\alpha_{v}, 0.001\right)}
$$

$N_{b}$ is in units of number of bubbles per cubic foot. It was formulated in this manner to capture the fact that coalescence of bubbles occurs as vapor increases in the flow. The model yields a bubble radius of 0.1 inches at a void fraction of 0.2 . The purpose of this model is to provide an interfacial area for the initiation of flashing.

The interfacial area for interfacial heat transfer coefficient calculations is:

$$
A_{i, s b}=\frac{6 \max \left(\alpha_{v}, 0.001\right) A_{x} \Delta X}{D_{b}}
$$

\subsubsection{Small-to-Large Bubble Regime}

The small-to-large bubble regime exists for void fractions ranging from $0.2-0.5$. The upper limit corresponds to the work of Taitel, Bornea, and Dukler [23]. Their work found that, considering spherical bubbles packed into a cubic lattice, bubbles begin to touch at a void fraction of 0.52 . This was assumed to represent the upper limit of bubbly flow.

This regime is considered to consist of a combination of small bubbles and large bubbles, as shown in Figure 3.1. Their distribution is determined by assuming that the void fraction of small bubbles stays constant at 0.2 , the upper limit for the small bubble flow regime, with the remainder of vapor void existing in the form of large bubbles. The large bubble size increases with void fraction until its diameter equals the channel size or 3 inches, whichever is less. The large bubbles are also considered to be spherical in shape when calculating interfacial area.

The small bubble regime calculations, as described in Section 3.2.2, are performed assuming all of the vapor is in the form of small bubbles and the interfacial area is stored as a temporary variable. Calculations assuming that all of the vapor is in the form of large bubbles are performed next. The results for the two different bubble types are then interpolated.

The large bubble radius is first calculated as follows: 


$$
r_{l b}^{*}=\left[0.2387\left(\alpha_{v}-\frac{\alpha_{s b} \alpha_{l}}{1-\alpha_{s b}}\right) A_{x} \Delta X\right]^{0.3333}
$$

The large bubble radius is calculated by subtracting the small bubble volume from the total vapor volume to get the large bubble volume, and then assuming spherical geometry of the large bubbles to calculate large bubble radius (see Box 1 for more details). The result is compared to the channel hydraulic diameter, the small bubble radius, and a limiting factor in the following axiom:

$$
r_{l b}=\max \left\{\operatorname { m i n } \left\{\begin{array}{c}
0.25 \mathrm{ft} \\
0.5 D_{h} \\
r_{l b}^{*} \\
r_{s b}
\end{array}\right.\right.
$$

For large bubbles, the interfacial area in a computational cell is equal to the surface area of a single bubbles times the number of bubbles in the cell. The number of large bubbles in a mesh cell is equal to the total large bubble volume divided by the single large bubble volume (the two values will clearly be equal if only one large bubble exists):

$$
N_{l b}=\frac{V_{l b}}{\frac{4}{3} \pi r_{l b}^{3}}
$$

Assuming that all vapor is in the form of large bubbles and multiplying this by the surface area of a single large bubble, the surface area of the large bubbles is:

$$
A_{i, l b}=\frac{\alpha_{v} A_{x} \Delta X}{\frac{4}{3} \pi r_{l b}^{3}} 4 \pi r_{l b}^{2}
$$

Simplifying this leads to:

$$
A_{i, l b}=3 \alpha_{v} A_{x} \frac{\Delta X}{r_{l b}}
$$

This expression is used in the calculation of large bubble interfacial drag coefficient.

To prevent a dependence on the adjacent void fraction, $\alpha_{v}$ of cells $(i, j)$ and $(i, j+1)$ is compared:

$$
A_{i, l b}^{*}=\frac{\min \left(\alpha_{v}(i, j), \alpha_{v}(i, j+1)\right)}{\alpha_{v}} A_{i, l b}
$$

With the small bubble interfacial area, calculated in Section 3.2.2 (Equation 3.13), and the large bubble interfacial area, presented in Equation 3.19, the interfacial area for the small-to-large bubble flow regime is calculated by interpolation. 


$$
A_{i, s l b}=F_{s b} A_{i, s b}+\left(1-F_{s b}\right) A_{i, l b}^{*}
$$

$F_{s b}$ is the ratio of vapor volume in the form of small bubbles to the total volume of vapor in the cell:

$$
F_{s b}=\frac{V_{s b}}{V_{c}}
$$

Using the definition of $V_{s b}$, which was calculated in Equation 3.26 during the large bubble radius derivation in Box 1, we can expand this to:

$$
F_{s b}=\frac{\alpha_{s b} \alpha_{l}}{\left(1-\alpha_{s b}\right) \alpha_{v}}
$$

\section{Box 1: Calculation of Large Bubble Radius}

The large bubble radius can be calculated from the large bubble volume if we assume spherical geometry:

$$
V_{l b}=\frac{4}{3} \pi r_{l b}^{* 3}
$$

The total large bubble volume is simply equal to the total volume minus the small bubble volume:

$$
V_{l b}=V_{v}-V_{s b}
$$

We calculate $V_{s b}$ by considering that the small bubble void, $\alpha_{s b}$, is the small bubble volume divided by the sum of liquid and small bubble volume.

$$
\alpha_{s b}=\frac{V_{s b}}{V_{l}+V_{s b}}
$$

Solving for $V_{s b}$, we get the following:

$$
V_{s b}=\frac{\alpha_{s b} V_{l}}{1-\alpha_{s b}}
$$

For a given momentum mesh cell with cross-sectional area $A_{x}$ and height $\Delta X$, the liquid volume is:

$$
V_{l}=\alpha_{l} A_{x} \Delta X
$$


Substituting Equation 3.27 into Equation 3.26, we are left with the small bubble volume in terms of known variables (since it was assumed that small bubble void was at the upper limit for the small bubble flow regime, $\left.\alpha_{s b}=0.2\right)$ :

$$
V_{s b}=\frac{\alpha_{s b} \alpha_{l} A_{x} \Delta X}{1-\alpha_{s b}}
$$

Substituting this into Equation 3.24 along with the definition of total vapor volume, the large bubble volume is:

$$
V_{l b}=\left(\alpha_{v}-\frac{\alpha_{s b} \alpha_{l}}{1-\alpha_{s b}}\right) A_{x} \Delta X
$$

Assuming that the total large bubble volume is all in a single large bubble, the large bubble radius is calculated by equating this equation to Equation 3.23 and solving for $r_{l b}^{*}$ (result shown in Equation 3.14). If total large bubble volume is, in fact, spread amongst several large bubbles, the limit presented in Equation 3.15 will act to reduce the large bubble radius.

\subsubsection{Churn/Turbulent Flow Regime}

The churn/turbulent region exhibits behaivor of large bubble flow, but is more chaotic in nature. In CTF, its behavior is captured by blending the small-to-large bubble regime and the annular/mist regime characteristics. The beginning of the churn/turbulent regime occurs at a void fraction of 0.5 and exists until a stable film forms, at which point transition to annular/mist occurs. Establishment of a stable film is dependent on channel geometry and vapor velocity, but it will occur no lower than a void fraction of 0.8 .

The transition from churn/turbulent to annular/mist is determined based on flow conditions and channel geometry. The void fraction used to demarcate the churn/turublent and annular/mist regimes - referred to as the critical void fraction - is a constant value of 0.8 .

Since the churn/turbulent regime is a mixture of small-to-large bubble flow regime properties and annular/ mist properties, calculations are performed for both flow regimes if $\alpha$ is between $\alpha=0.5$ and $\alpha=\alpha_{\text {crit }}$. Calculations for the interfacial area of the small-to-large bubble regime were presented in Section 3.2.3. Calculations for interfacial area of the annular/mist regime are presented next in Section 3.2.5.

There is actually no interpolation performed for the interfacial area term in the source code. Instead, the interfacial drag and heat transfer terms are calculated for the small-to-large bubble and annular/mist regimes and those terms are then interpolated using the $F_{a}$ interpolation factor, shown below:

$$
F_{a}=\frac{\alpha_{v}-0.5}{\alpha_{\mathrm{crit}}-0.5}
$$


The term is used for calculating churn/turbulent closure terms, as follows:

$$
P_{\mathrm{CT}}=F_{a} P_{\mathrm{AM}}+\left(1-F_{a}\right) P_{\mathrm{SLB}}
$$

$P$ denotes the term being calculated (i.e. either interfacial drag or interfacial heat transfer) and CT, AM, and SLB represent the corresponding closure term for the churn/turbulent, annular/mist, and small-to-large bubble regimes, respectively.

\subsubsection{Annular/Mist Flow Regime}

The annular/mist flow regime is characterized by a continuous liquid film surrounding a vapor core that may or may not contain a droplet field. The droplet diameter is calculated using the droplet interfacial area, which is calculated in the POST3D subroutine using the interfacial area transport equation, later discussed in Section 3.4. The droplet diameter is also limited, as shown below:

$$
D_{d}=\max \left\{\begin{array}{c}
\frac{6 \alpha_{e}}{A_{i, d}^{\prime \prime \prime}} \\
0.0005 \mathrm{ft}
\end{array}\right.
$$

The droplet diameter is later used for calculation of the interfacial drag on the droplet field. It is necessary to calculate the interfacial area for both the continuous liquid and droplet fields separately and then add them together. The interfacial area of the droplet field is very simply:

$$
A_{i, d}=A_{i, d}^{\prime \prime \prime} A_{x} \Delta X
$$

The interfacial area of the film is calculated by assuming that the vapor core is cylindrical in shape and then calculating its surface area[24]. The result is:

$$
A_{i, f}=\frac{4 \sqrt{\alpha_{v}+\alpha_{e}} A_{x} \Delta X}{D_{h}}
$$

To keep the transition between adjacent mesh cells smooth, Equation 3.34 is multiplied by a ramp, as follows:

$$
\begin{aligned}
A_{i, f} & =\sqrt{\frac{\alpha^{*}}{\alpha_{v}+\alpha_{e}}}\left(\frac{4 \sqrt{\alpha_{v}+\alpha_{e}} A_{x} \Delta X}{D_{h}}\right), \text { where } \\
\alpha^{*} & =\min \left\{\begin{array}{c}
1-\alpha_{l} \\
1-\alpha_{l}(i, j+1)
\end{array}\right.
\end{aligned}
$$




\subsection{Hot Wall Flow Regimes}

\subsubsection{Introduction}

Flow regimes are significantly different from those previously described in Section 3.2 when critical heat flux (CHF) is exceeded. This occurs in PWRs during accident conditions, like the blowdown phase of a large-break LOCA. Because departure from nucleate boiling (DNB) occurs, the vapor keeps the liquid from contacting the heated surfaces. The flow regimes recognized in the hot wall map are:

- Inverted annular flow

- Inverted slug flow

- Dispersed droplet flow

- Falling film flow

- Top deluge flow

These flow regimes are depicted graphically in Figure 3.3. The logic for selecting the flow regime is given in Figure 3.4.

\subsubsection{Inverted Annular Flow Regime}

This regime consists of an annular film of vapor surrounding a liquid core. It is determined to exist if the maximum wall temperature exceeds $T_{\mathrm{CHF}}$, if there is a hot wall regime in the mesh cell above $($ i.e. $(i, j+1))$, and if the liquid is subcooled.

The interfacial area of the liquid core is calculated assuming that it is cylindrical in shape:

$$
A_{i, f}^{\prime \prime \prime}=\frac{P_{w} \sqrt{\alpha_{l}}}{A_{x}}
$$

This can be rewritten in terms of hydraulic diameter as:

$$
A_{i, f}^{\prime \prime \prime}=\frac{4 \sqrt{\alpha_{l}} A_{x} \Delta X}{D_{h}}
$$

The droplet interfacial area is determined by the interfacial area transport equation, later presented in Section 3.4 . 


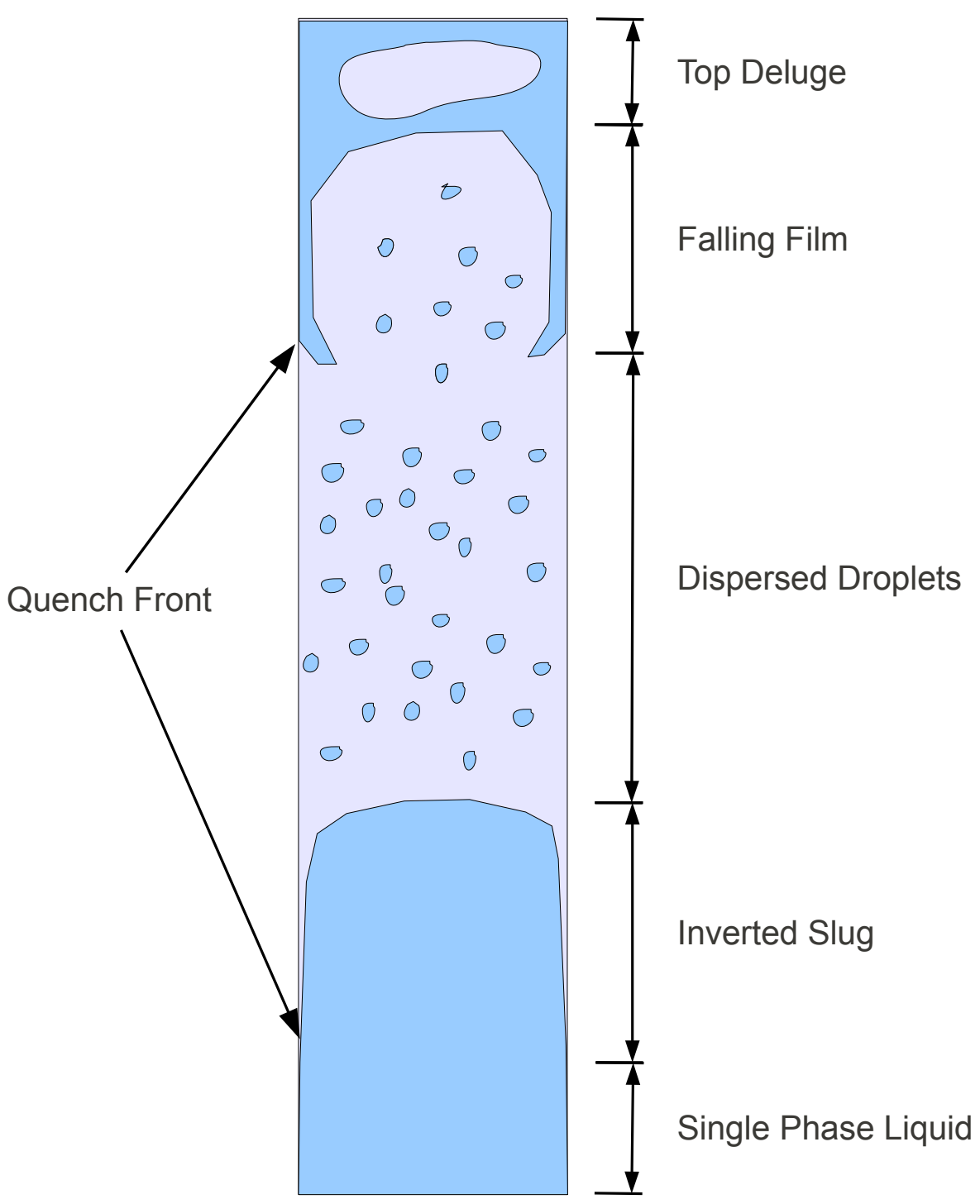

Figure 3.3: CTF hot wall flow regimes 


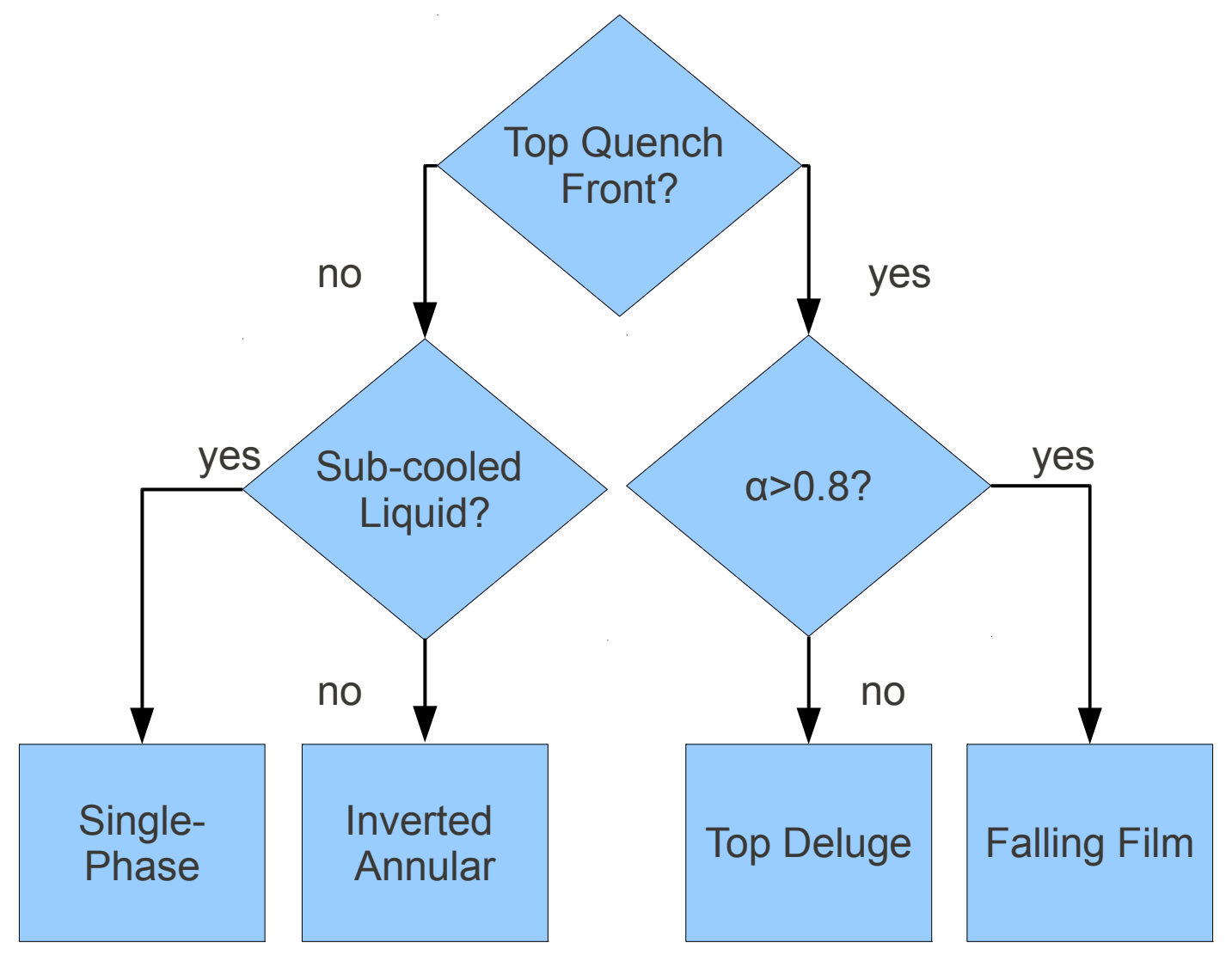

Figure 3.4: CTF hot wall flow regime selection logic 


\subsubsection{Dispersed Droplet Flow Regime}

The dispersed droplet field consists of a continuous vapor field containing entrained droplets throughout. Dispersed droplets can exist in all hot wall flow regimes.

Upon determining to use the hot wall flow regime map, calculations are done to determine droplet entrainment and interfacial area in INTFR, since dispersed droplets can exist in all hot wall flow regimes. The droplet interfacial area is obtained from solution of the interfacial area transport equation, solved in POST3D, and discussed in Section 3.4. The interfacial area for a mesh cell is:

$$
A_{i, d}=A_{i, d}^{\prime \prime \prime} A_{x} \Delta X
$$

\subsubsection{Falling Film Regime}

The falling film regime is one result of the existence of a top quench front. The regime consists of film flowing down and vapor flowing up. For it to exist, the mesh cell should be a hot wall flow regime with a normal wall flow regime above and the void should be greater than 0.8 .

The interfacial area for the film is calculated in the same way as in the normal wall regime, annular/mist:

$$
A_{i, f}^{\prime \prime \prime}=\frac{4 \sqrt{1-\alpha_{v}}}{D_{h}}
$$

The droplet interfacial area is, again, determined from the solution of the interfacial area transport equation:

$$
A_{i, d}=A_{i, d}^{\prime \prime \prime} A_{x} \Delta X
$$

\subsubsection{Top Deluge Flow Regime}

The top deluge regime consists of large liquid slugs, with a diameter equal to the channel hydraulic diameter. It, like the falling film regime, results from the existance of a top quench front (i.e. the mesh cell is in the hot wall regime with a normal wall regime in the mesh cell above). The regime occurs when void fraction is below 0.8 .

The interfacial area of the slugs is calculated assuming that the slugs are spherical in geometry. The interfacial area will then be the number of slugs in the volume times the interfacial area of each sphere:

$$
A_{i, s}^{\prime \prime \prime}=N_{s}^{\prime \prime \prime} \pi D_{s}^{2}
$$

$D_{s}$ is the diameter of a slug; however, we assume that the slug diameter is equal to the hydraulic diameter. The number of slugs is calculated by dividing the total liquid volume by the volume of one slug: 


$$
N_{s}^{\prime \prime \prime}=\frac{\alpha_{l}}{\frac{\pi D_{h}^{3}}{6}}
$$

Substituting Equation 3.42 into Equation 3.41 yields:

$$
A_{i, s}=\frac{6 \alpha_{l}}{D_{h}} A_{x} \Delta X
$$

The interfacial area of the droplet field is, once again, determined by the solution of the interfacial area transport equation:

$$
A_{i, d}=A_{i, d} A_{x} \Delta X
$$

\subsection{Interfacial Area Transport Equation}

The interfacial area transport equation tracks the interfacial area of the droplet field throughout the flow field. The equation is written as:

$$
\frac{d A_{i, d}^{\prime \prime \prime}}{d t}+\nabla \cdot\left(A_{i, d}^{\prime \prime \prime} \vec{U}_{e}\right)=A_{i, E}^{\prime \prime \prime}+A_{i, \Gamma}^{\prime \prime \prime}
$$

The terms on the LHS represent the rate of change in interfacial area with respect to time and the efflux of interfacial area through mesh cell connections. The RHS terms include the generation of interfacial area due to entrainment and evaporation.

The interfacial area transport equation is performed in POST3D after back-substitution is performed for the Jacobian matrices. Equation 3.45 is first multiplied through by the cell volume and the efflux term is put into discrete form:

$$
\frac{d A_{i, d}^{\prime \prime \prime}}{d t} V_{c}+\sum_{j}\left(A_{i, d}^{\prime \prime \prime} U_{e} A\right)_{j}=A_{i, E}+A_{i, \Gamma}
$$

The change in droplet volume due to evaporation is obtained from a mass balance:

$$
N_{d} \frac{d V_{d}}{d t}=\frac{-\eta \Gamma}{\rho_{l}}
$$

$N_{d}$ is the number of drops in the cell, $V_{d}$ is the droplet volume, and $\eta \Gamma$ is the evaporation rate from droplets, discussed in Section 2.2.2. The total entrained field volume divided by a single droplet volume gives the number of droplets: 


$$
N_{d}=\frac{\alpha_{e} V_{c}}{V_{d}}
$$

Substituting this into Equation 3.47 and putting the temporal term in discreet form gives:

$$
\frac{\alpha_{e} V_{c}}{V_{d}} \frac{V_{d}-V_{d}^{n}}{\Delta t}=-\frac{\eta \Gamma}{\rho_{l}}
$$

Simplifying this gives:

$$
\frac{V_{d}^{n}}{V_{d}}=1+\frac{\eta \Gamma \Delta t}{\rho_{l} \alpha_{e} V_{c}}
$$

We can equate the volume of a sphere to its surface area, as follows:

$$
\frac{V_{d}^{n}}{V_{d}}=\left(\frac{A_{i, d}^{n}}{A_{i, d}}\right)^{\frac{3}{2}}
$$

Substituting Equation 3.51 into Equation 3.50 yields:

$$
A_{i, d}^{\prime \prime \prime}=A_{i, d}^{n}{ }^{\prime \prime \prime}\left(\frac{1}{1+\frac{\eta \Gamma \Delta t}{\rho_{l} \alpha_{e} V_{c}}}\right)^{\frac{2}{3}}
$$

or:

$$
A_{i, d}^{\prime \prime \prime}=A_{i, d}^{n}{ }^{\prime \prime \prime}\left(\frac{\rho_{l} \alpha_{e} V_{c}}{\rho_{l} \alpha_{e} V_{c}+\eta \Gamma \Delta t}\right)^{\frac{2}{3}}
$$

Substituting Equation 3.53 into Equation 3.46 yields the following:

$$
A_{i, d}^{\prime \prime \prime}=A_{i, d}^{n \prime \prime \prime}\left(\frac{\rho_{l} \alpha_{e} V_{c}}{\rho_{l} \alpha_{e} V_{c}+\eta \Gamma \Delta t}\right)^{\frac{2}{3}}+\left\{\sum_{j}\left(A_{i, d}^{n}{ }^{\prime \prime \prime} u_{e} A\right)_{j}+A_{i, e}\right\} \frac{\Delta t}{V_{c}}
$$

Limits are placed on this final form of the equation when calculating the new time interfacial area:

$$
A_{i, d}^{\prime \prime \prime}=\min \left\{\operatorname { m a x } \left\{\begin{array}{c}
A_{i, d}^{\prime \prime \prime} \text { from Equation } 3.54 \\
\frac{10^{-5}}{A_{x} \Delta X} \\
12,000 \alpha_{e}
\end{array}\right.\right.
$$




\section{Macro-Mesh Cell Closure Models}

\subsection{Introduction}

The conservation equations that have been discussed in Chapter 2 contain terms that must be defined prior to a solution of the conservation equations. This chapter discusses those closure terms which affect the overall mesh cell, dubbed the "macro-mesh cell" closure terms. These closure terms include the wall shear stress, inter-cell shear (e.g. caused by liquid/vapor interface between cells), wall heat transfer, turbulent mixing, and void drift. Each of these terms is discussed in the following sections.

\subsection{Wall Shear and Form Loss Models}

The wall shear model was briefly introduced in Section 2.3.2.2 during the discussion of the finite-differenced form of the momentum conservation equations. The wall shear term is restated here as a coefficient times the field mass flow rate.

$$
\tau_{w, x}=k_{w, x} \dot{m} \Delta X
$$

The wall shear, in CTF, is also visualized as a combination of frictional losses and flow form losses, as follows:

$$
\left.\left.\tau_{w, x}=\left[\frac{d P}{d X}\right)_{\text {fric }}+\frac{d P}{d X}\right)_{\text {form }}\right] \Delta X
$$

Combining this equation with Equation 2.43 allows for the definition of the wall drag coefficient $K_{w, x}$ :

$$
\left.\left.K_{w, x}=\frac{d P}{d X}\right)_{\text {fric }} \frac{1}{\dot{m}}+\frac{d P}{d X}\right)_{\text {fric }} \frac{1}{\dot{m}}
$$

To further this derivation of the wall drag loss coefficient, we define the friction and form pressure loss components in the following sub-sections. 


\subsubsection{Friction Loss Model}

PSU RDFMG version of COBRA-TF (CTF) uses a two-phase pressure drop model, based on the work of Wallis[25], as follows:

$$
\left.\frac{d P}{d X}\right)_{\text {fric }}=\frac{f_{w} G_{x}^{2}}{2 D_{h} \rho} \Phi^{2}
$$

$G_{x}$ is the mass flux of the field of interest and $\Phi$ is a two-phase multiplier which is defined as follows:

$$
\Phi= \begin{cases}1 / \alpha_{l} & \text { for normal wall conditions } \\ 1 / \alpha_{v} & \text { for hot wall conditions }\end{cases}
$$

The friction factor $f_{w}$ is defined using phase Reynolds number $R e$ and surface roughness $\epsilon$. The friction factor correlations are in Table 1. Note that all correlations, except the Churchill correlation, return the Darcy friction factor. The Churchill correlation returns the Fanning friction factor, which is four times smaller than the Darcy friction factor. Since the CTF model uses the Darcy friction factor, the Fanning friction factor is multiplied by 4 .

The phase Reynolds number is based on phasic properties:

$$
R e=\frac{D_{h}\left|G_{k}\right|}{\mu_{k}}
$$

The subscript, $k$, represents the phase, and can be either liquid or vapor. Addition of surface roughness requires a special treatment of calculating the frictional pressure drop when there exists different rod types in the same subchannel with different surface roughnesses. CTF determines a surface-area-weighted average surface roughness for the channel.

$$
\epsilon_{i, \mathrm{eff}}=\frac{\sum_{r} \epsilon_{r} P_{w, r}}{P_{w, i}}
$$

In the equation, the $r$ subscript is a counter over the rod surfaces touching the channel $i$. The term, $P_{w, r}$, is the wetted perimeter of the rod segment that touches the channel and $\epsilon_{r}$ is the roughness of that rod segment. The denominator, $P_{w, i}$, is the total wetted perimeter of Channel $i$.

It is important to note that the sum of $P_{w, r}$ over all rod surfaces touching the channel does not necessarily equal the total channel wetted perimeter, $P_{w, i}$. The user is free to set the channel wetted perimeter, $P_{w, i}$, to whatever they want, independent of the actual dimensions of the rods touching the channel. This feature is most commonly used for specifying a wall around a rod bundle without explicitly modeling the solid objects that make up the wall. In this way, the wall shear effect can be captured without actually modeling the wall. The surface roughness is only considered for rod and unheated conductor surfaces. Therefore, in a boundary channel of a rod bundle with a fuel canister, for example, the wall will always be assumed smooth, regardless of the roughness of the attached rod. 
Table 1: CTF friction factor correlations.

\begin{tabular}{|c|c|c|c|}
\hline $\begin{array}{l}\text { Original co } \\
f_{w}=\max \end{array}$ & $\begin{array}{l}\text { relation: } \\
\qquad 64.0 / R e \\
\max \left(1.691 R e^{-0.43}, 0.117 R e^{-0.14}\right)\end{array}$ & $\begin{array}{l}\text {, laminar } \\
\text {, turbulent }\end{array}$ & \\
\hline $\begin{array}{l}\text { McAdam's } \\
f_{w}=\max \{\end{array}$ & $\begin{array}{l}\text { orrelation:[26] } \\
\qquad 64.0 / R e^{-}, \text {laminar } \\
0.204 R e^{-0.2}, \text { turbulent }\end{array}$ & & \\
\hline $\begin{array}{l}\text { Zigrang-Syl } \\
f_{w}=\max \end{array}$ & $\begin{array}{l}\text { ester correlation:[27] } \\
\qquad 64.0 / \operatorname{Re} \\
\left(-2.0 \log \left(\frac{\epsilon}{3.7 D}+\frac{2.51}{\operatorname{Re}}\left[1.14-2 \log \left(\frac{\epsilon}{I}\right.\right.\right.\right.\end{array}$ & $\left.\left.\left.\left(+\frac{21.25}{R e^{0.9}}\right)\right]\right)\right)^{-2}$ & $\begin{array}{l}\text {, laminar } \\
\text {, turbulent }\end{array}$ \\
\hline $\begin{array}{l}\text { Churchill cc } \\
f_{f}=2\left[\left(\frac{8}{R e}\right)\right. \\
\text { where } \\
a=(2.475 l 7 \\
b=\left(\frac{3.753 x 1}{R e}\right.\end{array}$ & $\begin{array}{l}\text { rrelation }{ }^{a}:[28] \\
\left.2+\frac{1}{(a+b)^{3 / 2}}\right]^{1 / 12} \\
\left.\left[\frac{7}{\left(\frac{7}{R e}\right)^{0.9}+0.27\left(\frac{\epsilon}{D}\right)}\right]\right)^{16} \\
-)^{16}\end{array}$ & & \\
\hline $\begin{array}{l}\text { User-define } \\
f_{w}=\max \end{array}$ & $\begin{array}{ll}\text { function }^{b}: & \\
64.0 / R e & , \text { laminar } \\
A+B R e^{C} & \text {, turbulent }\end{array}$ & & \\
\hline
\end{tabular}

${ }^{a}$ Applies to all three flow regimes; laminar, transition and turbulent.

${ }^{b} \mathrm{~A}, \mathrm{~B}$ and $\mathrm{C}$ are user-defined coefficients. 
Additionally, the effect of wall drag in the lateral momentum cell is also considered in gaps that touch a wall. However, walls that touch gaps are also considered smooth.

\subsubsection{Form Loss Model}

The form pressure drop is defined as follows:

$$
\left.\frac{d P}{d X}\right)_{\text {form }}=\alpha_{k} \frac{K_{x}}{2 \Delta X} \rho_{k}\left|U_{k}\right| U_{k}
$$

Again, $k$ represents the phase but, in this case, can be either liquid, vapor, or entrained droplets and $U$ is the field velocity. Note that, while frictional loss is only calculated for liquid and vapor fields, the form loss is experienced and calculated for all three fields. $K_{x}$ is the form loss coefficient which may be user-supplied, code-calculated, or a combination of both, depending on the modeling circumstances. In INTFR, $K_{x}$ is either user-supplied or code-calculated.

The flow blockage coefficient, if chosen to be code-modeled, is calculated by the following equation:

$$
K_{\text {grid }}=\min \left(20,196 R e_{\text {mix }}^{-0.333}\right) f_{\text {loss }} A_{\text {block }}^{2}
$$

$f_{\text {loss }}$ is a user-defined pressure loss coefficient multiplier, $A_{\text {block }}$ is the user-defined ratio of blocked area to flow area, and $R e_{\text {mix }}$ is the droplet/bubbles mixture Reynolds number, defined by Equation 4.9.

$$
\begin{aligned}
R e_{\text {mix }} & =\frac{G_{T} D_{h}}{\mu_{\text {mix }}}, \text { where } \\
\mu_{\text {mix }} & =\min \left(\mu_{\text {bubbles }}, \mu_{\text {drops }}\right), \text { and } \\
\mu_{\text {bubbles }} & =\mu_{l}\left(1-\alpha_{v}\right)^{a}, \quad \text { where } \\
a & =\frac{-2.5\left(\mu_{v}+0.4 \mu_{l}\right)}{\mu_{v}+\mu_{l}} \\
\mu_{\text {drops }} & =\mu_{v} \alpha_{v}^{b}, \quad \text { where } \\
b & =\frac{-2.5\left(\mu_{l}+0.4 \mu_{v}\right)}{\mu_{v}+\mu_{l}}
\end{aligned}
$$

\subsubsection{Simplification of Wall/Form Loss Model}

The friction pressure loss term of Equation 4.2 is defined separately in the INTFR subroutine; however, the form loss term has simplifications made during its formulation. Therefore, Equation 4.2 is reformulated using the substitution of Equation 4.7: 


$$
\left.K_{w, x}=\frac{d P}{d X}\right)_{\text {fric }} \frac{1}{\dot{m}}+\frac{\alpha_{k} \rho_{k} U_{k} K_{x}\left|U_{k}\right|}{2 \Delta X \dot{m}}
$$

It is evident that the mass flow rate term, $\dot{m}$, can be canceled with some of the numerator terms in the form loss term; however a simplification is made in the source code at this point which can be a significant source of confusion when analyzing the source code. Recall that the wall drag loss coefficient, $K_{w, x}$, is multiplied by $\dot{m} \Delta X$ in order to represent the wall shear stress (units of force (see Equation 2.43)). We solve the momentum equations in order to calculate the field mass flow rates, $\dot{m}$. Returning to Equation 2.42, which is the discretized form of the Cartesian x-momentum equation, there are mass flow rate terms on both the LHS and RHS of the equation. On the LHS the mass flow term is multiplied by $\left(\Delta X A_{x}\right) / \Delta t$, which is carried over to the RHS during the solution process. As a result, the wall drag loss coefficient, in the XSCHEM subroutine, is multiplied by $\Delta t / \Delta X$. The cross-sectional area does not appear in XSCHEM because it is eliminated with the cross-sectional area term that appears in INTFR. Therefore, when further simplifying Equation 4.10, we take care to move the cross-sectional area back to the LHS, as follows:

$$
\left.K_{w, x} A_{x}=\frac{d P}{d X}\right)_{\text {fric }} \frac{A_{x}}{\dot{m}}+\frac{K_{x}\left|U_{k}\right|}{2 \Delta X}
$$

Now, the $A_{x}$ on the LHS of Equation 4.11 will cancel with the $A_{x}$ on the LHS of Equation 2.42, which is why these $A_{x}$ terms never appear in the INTFR or XSCHEM subroutines and why the wall drag loss coefficient takes the form of Equation 4.11. To avoid any additional confusion, $K_{w, x}$ is multiplied by $\Delta X$ first in XSCHEM (upon being received from INTFR), and then later multiplied by $\Delta t / \Delta X$ (from the LHS of the discrete form of the momentum equation) during the momentum equation solution.

The above equations were presented with regards for flow in the axial direction, but a wall drag loss coefficient is also calculated for the transverse direction. In this case, mass flow rates, field velocities and geometry parameters are with regards to the transverse direction. The phase Reynolds number, for example, is calculated as shown in Equation 4.12.

$$
R e_{k}=\frac{G_{z, k}\left(\frac{2 L_{g}}{F_{w}}\right)}{\mu_{k}}
$$

The hydraulic diameter was represented in terms of the transverse distance between sub-channels, $L_{g}$, and the wetted wall multiplier, $F_{w}$, which is a user-defined parameter that declares the number of wetted walls in a transverse momentum cell. The $F_{w}$ term also appears in the frictional pressure drop term, as shown in Equation 4.13, used along with the $L_{g}$ term to represent hydraulic diameter.

$$
\left.\frac{d P}{d Z}\right)_{\text {fric }, k}=\frac{F_{w} f_{w k} G_{z, k}^{2}}{4 L_{g} \bar{\rho}_{k}}
$$

With $F_{w}$ set to zero, no frictional pressure drop will be calculated in the transverse direction. With $F_{w}$ set to 0.5 , frictional pressure drop is calculated for an area equal to $\Delta X \Delta Z$, which is one side of a lateral momentum cell. 
Pertaining to the form loss coefficient, there is no option to use a code calculated value-the form loss coefficient in the lateral direction must be user-supplied. A value of 0.5 is recommended for flow across a single bank of tubes. For gaps that are defined to model more than one physical gap, the number of modeled physical gaps should be multiplied by 0.5 . The transverse gap loss coefficients are defined on Card Group 3 of the input deck.

In CTF, the liquid phase is assumed to carry all of the wall drag in the normal flow regimes unless void fraction is very high. The reverse is true for hot wall flow regimes, with the vapor phase carrying all the wall drag. To accomplish this desired behavior in the code, the frictional pressure drop term of Equation 4.11 is multiplied by multiplication factors, $F_{l}$ (for the liquid phase wall drag loss coefficient) and $F_{v}$ (for the vapor phase wall drag loss coefficient). The equations for liquid and vapor phase wall drag loss coefficients are shown in Equation 4.14.

$$
\begin{aligned}
K_{w, x, l} & \left.=\frac{d P}{d X}\right)_{\text {fric }, l} \frac{A_{x}}{\dot{m}_{l x}} F_{l}+\frac{K_{x}\left|U_{l}\right|}{2 \Delta X} \\
K_{w, x, v} & \left.=\frac{d P}{d X}\right)_{\text {fric }, v} \frac{A_{x}}{\dot{m}_{v x}} F_{v}+\frac{K_{x}\left|U_{v}\right|}{2 \Delta X}
\end{aligned}
$$

The terms, $F_{l}$ and $F_{v}$, are defined as follows:

$$
\begin{aligned}
& F_{l}=\left\{\begin{array}{cl}
\left(1-F_{\mathrm{spv}}\right) & \text { for normal wall regimes } \\
F_{\mathrm{spl}} & \text { for hot wall regimes }
\end{array}\right. \\
& F_{v}=\left\{\begin{array}{cl}
F_{\mathrm{spv}} & \text { for normal wall regimes } \\
\left(1-F_{\mathrm{spl}}\right) & \text { for hot wall regimes }
\end{array}\right.
\end{aligned}
$$

$F_{\text {spv }}$ and $F_{\text {spl }}$ are defined as follows:

$$
\begin{gathered}
F_{\mathrm{spv}}=\max \left\{\operatorname { m i n } \left\{\begin{array}{c}
1.0 \\
\frac{\alpha_{v}-0.9990}{0.9999-0.9990} \\
0.0
\end{array}\right.\right. \\
F_{\mathrm{spl}}=\max \left\{\operatorname { m i n } \left\{\begin{array}{c}
1.0 \\
\frac{\alpha_{v}-0.001}{0.0001-0.001} \\
0.0
\end{array}\right.\right.
\end{gathered}
$$

Some analysis of Equation 4.16 reveals that, except for very high void fractions over $0.999, F_{\mathrm{spv}}$ will be zero. If the cell is in a normal wall flow regime, $F_{l}$ will be 1 , and $F_{v}$ will be 0 , leading to all wall drag being carried by the liquid phase. Conversely, $F_{\text {spl }}$ will be 1 for all instances except when void fraction is very low (less than 0.001 ). If the cell is in a hot wall regime, $F_{v}$ will be 1 and $F_{l}$ will be 0 , leading to all wall drag being carried by the vapor phase. 
The calculation of $F_{l}$ and $F_{v}$ differs slightly for the transverse momentum cell solution. Consideration is made for the fact that flow can go from a cell having a normal wall flow regime to a cell having a hot wall flow regime. If either of the continuity cells that the transverse momentum cell connects to in a hot wall flow regime, the $F_{\mathrm{spl}}$ ramping factor will be used over the $F_{\mathrm{spv}}$ ramping factor. This is demonstrated in Equation 4.17 .

$$
\begin{aligned}
& F_{l}=\left\{\begin{array}{cl}
\left(1-F_{\mathrm{spv}}\right) & \text { both cells in normal wall flow regime } \\
F_{\mathrm{spl}} & \text { at least one cell in hot wall flow regime }
\end{array}\right. \\
& F_{v}=\left\{\begin{array}{cl}
F_{\mathrm{spv}} & \text { both cells in normal wall flow regime } \\
\left(1-F_{\mathrm{spl}}\right) & \text { at least one cell in hot wall flow regime }
\end{array}\right.
\end{aligned}
$$

\subsection{Inter-cell Drag Models}

The inter-cell drag model exists to account for inter-phase drag that may occur between mesh cells should there be a large void fraction gradient. Typical causes for the existence of inter-cell interfacial drag include areas where liquid can pool (e.g. upper core tie plate) and channels that contain a vapor jet. CTF will check for large void fraction gradient interfaces between axially-connected mesh cells (horizontal interface) and between transversally-connected mesh cells (vertical interface).

The mesh void fraction gradient is checked in the INTFR subroutine to detect interfaces. If one exists, calculations of the inter-cell interfacial drag is also calculated in INTFR. To illustrate the model, consider two adjacent cells, $a$ and $b$. CTF declares an interface to exist when $\alpha_{i}>0.8$ and $\alpha_{j}<0.6$ - the cell void fractions could be opposite, with cell $a$ having the higher void fraction, but we assume here that cell $a$ is the vapor side and cell $b$ is the liquid side.

An interface can exit between cells that are laterally connected (vertical interface) or between cells that are vertically connected (horizontal interface). As an example, refer to Figure 4.1 where the blue cells represent continuity mesh cells, the red cells represent axial momentum cells, and the green cell represents a transverse momentum cell. A vertical interface could exist between continuity mesh cells $(i i, J)$ and $(j j, J)$ if $\alpha_{v}$ were less than 0.6 in one cell and greater than 0.8 in the other - the resulting inter-cell interfacial force would be applied to the adjacent axial momentum cells. A horizontal interface could exist between continuity mesh cells $(i i, J-1)$ and $(i i, J)$ if the correct void fraction gradient existed - the resulting inter-cell interfacial force would be applied to the adjacent transverse momentum cells.

The important thing to note in this example is that the inter-cell interfaces are defined using the void fractions of the continuity mesh cells; however, the resulting inter-cell interfacial forces are applied in the momentum cells. Since the momentum and continuity mesh cells are staggered, we must choose the interface area carefully (discussed during the "Model as Coded" section).

The drag force equation is used, with the relative velocity between the vapor ( $a$ side) and liquid ( $b$ side) being used for the velocity. The interfacial drag coefficient, $f_{i}$, is set to 0.08 and the average density between adjacent cells is used. The drag force equation for inter-cell interfacial drag is depicted in Equation 4.18 for both the vertical and lateral directions. 


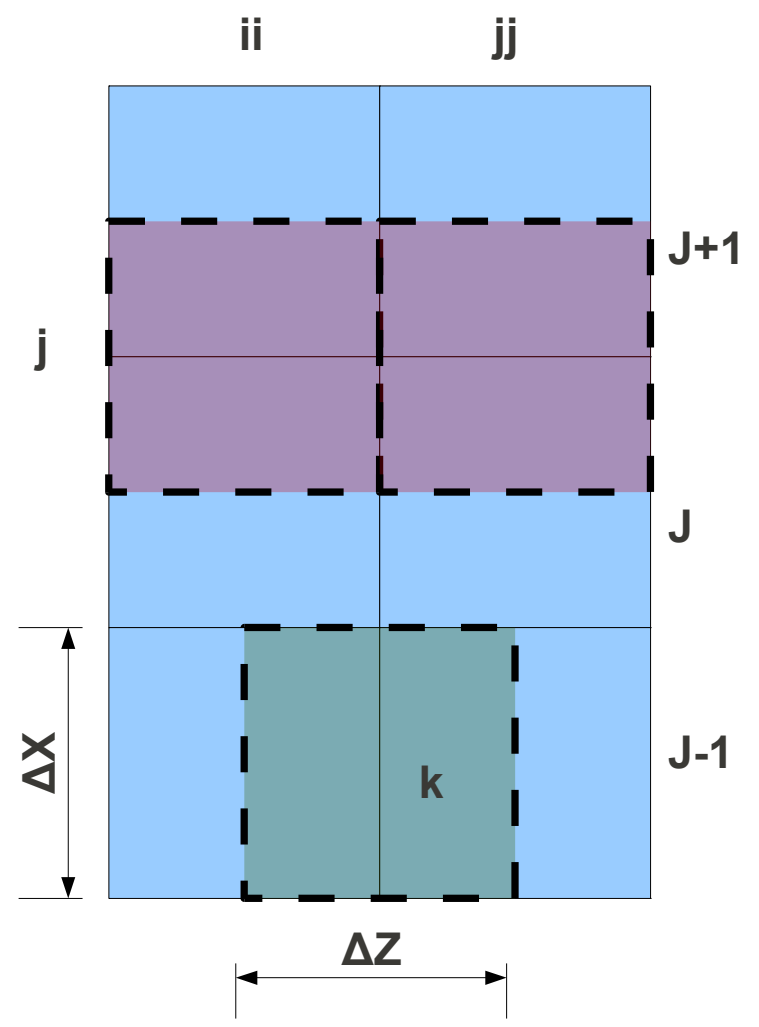

Figure 4.1: Example of meshing of two adjacent sub-channels, $i i$ and $j j$ 


$$
\begin{aligned}
& F_{I, x}=\frac{1}{2} f_{i} \bar{\rho}_{v}\left|\left(U_{v, a}-U_{l, b}\right)\right|\left(U_{v, a}-U_{l, b}\right) A_{I, x} \\
& F_{I, z}=\frac{1}{2} f_{i} \bar{\rho}_{v}\left|\left(W_{v, a}-W_{l, b}\right)\right|\left(W_{v, a}-W_{l, b}\right) A_{I, z}
\end{aligned}
$$

There was no explicit inter-cell interfacial drag term declared in the axial and transverse momentum equations of Chapter 2. In the source code, the inter-cell terms are summed up for each cell in the current axial level in INTFR. Consideration is made for which channel contains vapor and which contains liquid and, consequently, whether the inter-cell interfacial drag force should be added or subtracted from the total for a given cell. Note that when inter-cell interfacial drag force is added to cell $a$, the same force must be subtracted from the connected cell $b$. These values are then passed to XSCHEM where they are added to the explicit portion of the momentum equation solutions (see terms $A_{1}, A_{2}$, and $A_{3}$ in Equations $2.64-2.66$ ).

Upon finding an inter-cell phase interface, CTF calculates the inter-cell interfacial drag. For a vertical interface, the interface area used in Equation 4.18 is calculated as follows:

$$
A_{I, x}=\frac{1}{2} L_{g} \Delta X\left|\Delta \alpha_{l}\right|
$$

We refer back to Figure 4.1 in discussing this equation. $L_{g}$ is the gap width (the dimension of the green transverse momentum cell going in and out of the page) and $\Delta X$ is the gap height. The reason for multiplying this by $1 / 2$ is because, as previously discussed, the gap inter-phase interface is defined between continuity mesh cells. However, we add the inter-cell interfacial drag force to the momentum equations, which are solved for the momentum mesh cells. If, for example, an inter-cell interface exists between $(i i, J) /(j j, J)$, but not between $(i i, J+1) /(j j, J+1)$, the axial momentum cells, $(i i, j)$ and $(j j, j)$, should only experience inter-cell interfacial drag from continuity mesh cells $(i i, J) /(j j, J)$. Likewise, the inter-cell interfacial drag force between continuity mesh cells $(i i, J) /(j j, J)$ should be equally distributed between the axial momentum cells above and below.

The $\Delta \alpha_{l}$ term of Equation 4.19 represents the difference in liquid void fraction between the adjacent continuity mesh cells. We multiply by this term because, for an interfacial drag force, we are obviously only interested in the contact area of vapor and liquid phases. By taking the difference in liquid void fractions, we are estimating the contact area of the liquid and vapor phases in the adjacent cells.

For the case of a horizontal interface, the interface area is defined as:

$$
A_{I, z}=\frac{1}{2} L_{g} \Delta Z
$$

Note the absence of the liquid void fraction gradient term-liquid water is assumed to pool at the top or bottom of the adjacent cells, leading to total liquid/vapor contact across the entire interface. 


\subsection{Turbulent Mixing and Void Drift}

In this section, we will define the source terms in the CTF governing equations that act to capture turbulent mixing and void drift. The turbulent mixing terms are modeled by a simple diffusion approximation using mixing length theory. They act only in the lateral directions (i.e. in the gaps of the model) between adjacent subchannels. As stated in Todreas and Kazimi[29], the net two-phase turbulent mixing of mass between two subchannels can be captured using the following relationship:

$$
W_{i j}^{D^{\prime}}=\epsilon \frac{s_{i j}}{z_{i j}^{T}}\left(\rho_{f}-\rho_{g}\right)\left[\alpha_{v, j}-\alpha_{v, i}-\left(\alpha_{v, j}-\alpha_{v, i}\right)_{\text {equil }}\right]
$$

This is an equal-volume model, meaning that equal volumes of fluid are mixed between adjacent subchannels, as opposed to mixing equal masses of fluid. In this equation, $\epsilon$ and $z_{i j}^{T}$ are the eddy diffusivity and the turbulent mixing length, respectively. These terms result from mixing length theory being used to characterize turbulent exchange. CTF has models for defining the eddy diffusivity term, which will be discussed near the end of this section. The $s_{i j}$ term is simply the width of the gap between the adjacent subchannels. To simplify our notation going forward, we shall condense the term, $\epsilon \frac{s_{i j}}{z_{i j}^{T}}$ into the single variable, $V^{T}$.

There are two components inside the square brackets of the previous equation. The first, $\alpha_{v, j}-\alpha_{v, i}$, represents the turbulent mixing driving force. By this model, the two-phase turbulent mixing will act to drive the liquid mass to the higher void channel and the vapor mass to the lower void channel. In reality, this is indeed the observed trend, but only to a point. A second phenomenon, known as void drift, acts to drive vapor to the larger area subchannels. This effect is captured with the second term in the brackets, $\left(\alpha_{v, j}-\alpha_{v, i}\right)_{\text {equil }}$, which is known as the equilibrium void distribution. By subtracting this term from the first, the turbulence will mix the adjacent channel masses to a point of equilibrium, which is a balance of the two phenomena: turbulent mixing and void drift.

Because CTF uses a segregated flow solution (i.e. vapor and liquid governing equations are individually solved), it is necessary to separate Equation 4.21 into two components: one for vapor and one for liquid.

$$
\begin{gathered}
W_{i j, v}^{D^{\prime}}=-V^{T} \rho_{g}\left[\alpha_{v, j}-\alpha_{v, i}-\left(\alpha_{v, j}-\alpha_{v, i}\right)_{\text {equil }}\right] \\
W_{i j, l}^{D^{\prime}}=V^{T} \rho_{f}\left[\alpha_{v, j}-\alpha_{v, i}-\left(\alpha_{v, j}-\alpha_{v, i}\right)_{\text {equil }}\right]
\end{gathered}
$$

To simplify the derivation, we choose to further separate the above equations into individual terms for turbulent mixing and void drift. The vapor turbulent mixing and void drift of mass are show in Equations 4.24 and 4.25 , respectively.

$$
\begin{gathered}
W_{i j, v, T M}^{D^{\prime}}=-V^{T} \rho_{g}\left[\alpha_{v, j}-\alpha_{v, i}\right] \\
W_{i j, v, V D}^{D^{\prime}}=V^{T} \rho_{g}\left[\left(\alpha_{v, j}-\alpha_{v, i}\right)_{\text {equil }}\right]
\end{gathered}
$$


The equations for the liquid are as follows:

$$
\begin{gathered}
W_{i j, l, T M}^{D^{\prime}}=V^{T} \rho_{f}\left[\alpha_{v, j}-\alpha_{v, i}\right] \\
W_{i j, l, V D}^{D^{\prime}}=-V^{T} \rho_{f}\left[\left(\alpha_{v, j}-\alpha_{v, i}\right)_{\text {equil }}\right]
\end{gathered}
$$

At this point, we focus on turbulent mixing and void drift separately in the following two sub-sections to complete the derivation.

\subsubsection{Turbulent mixing}

Equations 4.24 and 4.26 have presented models for capturing turbulent mixing of vapor and liquid mass between subchannels, respectively. However, the turbulent mixing will also affect the other two fields in CTF: non-condensable gas and droplets. To obtain the droplet mass turbulent mixing equation, we perform some algebraic manipulation on the liquid mass turbulent mixing equation.

$$
W_{i j, l}^{D^{\prime}}=-V^{T} \rho_{f}\left[\left(1-\alpha_{v, j}\right)-\left(1-\alpha_{v, i}\right)\right]
$$

This is equivalent to the following:

$$
W_{i j, l}^{D^{\prime}}=-V^{T} \rho_{f}\left[\left(\alpha_{l, j}+\alpha_{e, j}\right)-\left(\alpha_{l, i}+\alpha_{e, i}\right)\right]
$$

We can then separate this into individual equations for the liquid and droplet fields, which are now in terms of the liquid and droplet void fractions.

$$
\begin{gathered}
W_{i j, l}^{D^{\prime}}=-V^{T} \rho_{f}\left[\alpha_{l, j}-\alpha_{l, i}\right] \\
W_{i j, e}^{D^{\prime}}=-V^{T} \rho_{f}\left[\alpha_{e, j}-\alpha_{e, i}\right]
\end{gathered}
$$

Next, we must consider the non-condensable gas. Since the non-condensable gas occupies the same volume as the vapor, it is derived from the vapor equation similar to how the droplet equation was pulled from the liquid equation. The details of the derivation are left out and the equation is presented below. One notable point of departure from the vapor equation is that the vapor density must be substituted by the non-condensable gas density, which is tracked separately in CTF.

$$
W_{i j, \mathrm{gas}}^{D^{\prime}}=-V^{T}\left[\rho_{\mathrm{gas}, j} \alpha_{v, j}-\rho_{\mathrm{gas}, \mathrm{i}} \alpha_{v, i}\right]
$$

Note that our model has now been reduced so that field mass - whether it is vapor, liquid, or droplet masswill always travel down the field gradient (e.g. vapor mass will move from the high to low vapor void channel, 
liquid mass will move from the high to low liquid void channel, and droplet mass will move from the high to low droplet void channel).

Up to this point, we have used the assumption that the phase densities are equal to the saturation densities, which are also constant through space. This assumption is not made in CTF; phase density is not necessarily equal to saturation density and it is also allowed to vary from cell-to-cell. Therefore, we replace the saturation densities with the true, space-dependant liquid and vapor densities in the vapor, liquid, and droplet turbulent mixing equation.

$$
\begin{gathered}
W_{i j, v}^{D^{\prime}}=-V^{T}\left[\rho_{v, j} \alpha_{v, j}-\rho_{v, i} \alpha_{v, i}\right] \\
W_{i j, l}^{D^{\prime}}=-V^{T}\left[\rho_{l, j} \alpha_{l, j}-\rho_{l, i} \alpha_{l, i}\right] \\
W_{i j, e}^{D^{\prime}}=-V^{T}\left[\rho_{l, j} \alpha_{e, j}-\rho_{l, i} \alpha_{e, i}\right]
\end{gathered}
$$

This completes the derivation of the 4 field turbulent mixing equations for mass; however, we can also mix energy and momentum. Rather than repeat the previous lengthy derivation, we note the transported quantities for turbulent mixing of energy and momentum and then present the final forms of the energy and momentum turbulent mixing equations, which conveniently have the same form as the mass equations. The transported quantity for the energy equation is $\rho h$ and the transported quantity for the momentum equation is $\rho V$. Note that we do not have turbulent mixing of non-condensable energy or momentum, so no equation is provided for the field. The energy turbulent mixing source terms are as follows:

$$
\begin{gathered}
W_{i j, v}^{H^{\prime}}=-V^{T}\left[\rho_{v, j} \alpha_{v, j} h_{v, j}-\rho_{v, i} \alpha_{v, i} h_{v, i}\right] \\
W_{i j, l}^{H^{\prime}}=-V^{T}\left[\rho_{l, j} \alpha_{l, j} h_{l, j}-\rho_{l, i} \alpha_{l, i} h_{l, i}\right] \\
W_{i j, e}^{H^{\prime}}=-V^{T}\left[\rho_{l, j} \alpha_{e, j} h_{l, j}-\rho_{l, i} \alpha_{e, i} h_{l, i}\right]
\end{gathered}
$$

The momentum mixing equations are derived using the mass flowrate term in CTF. By dividing this by the cell cross-flow area, we obtain $\alpha_{k} \rho_{k} V_{k}$, which is the momentum of Phase $k$.

$$
\begin{aligned}
& W_{i j, v}^{M^{\prime}}=-V^{T}\left[\frac{\dot{m}_{v, j}}{A_{j}}-\frac{\dot{m}_{v, i}}{A_{i}}\right] \\
& W_{i j, l}^{M^{\prime}}=-V^{T}\left[\frac{\dot{m}_{l, j}}{A_{j}}-\frac{\dot{m}_{l, i}}{A_{i}}\right] \\
& W_{i j, e}^{M^{\prime}}=-V^{T}\left[\frac{\dot{m}_{e, j}}{A_{j}}-\frac{\dot{m}_{e, i}}{A_{i}}\right]
\end{aligned}
$$


The previous derivation of the source terms was done for the sake of completeness. In reality, CTF makes certain simplifying assumptions and does not include all source terms in all cases. There are two possible scenarios in a simulation: (1) the flow regime is single-phase, small bubble, slug flow, or churn-turbulent, or (2) the flow regime is annular-mist or mist. In Case 1, there is no turbulent mixing in the droplet field, since there will not be a significant amount of droplets in such flow regimes. In Case 2, there is no turbulent mixing in the liquid field, since it will only exist as a thin film or not at all.

\subsubsection{Void Drift}

We begin from the void drift equations for vapor and liquid mass, which were presented as Equations 4.25 and 4.27. Note that there is no void drift impact on the droplets; however, there will be an impact on the non-condensable gas, which exists in the vapor space. For all void drift equations, it is necessary to define the equilibrium void distribution term, $\left(\alpha_{v, j}-\alpha_{v, i}\right)_{\text {equil }}$. To obtain a definition of this term, we begin with Lahey's derivation of the equilibrium density distribution[30]:

$$
\left(\left\langle\overline{\rho_{i}}\right\rangle-\left\langle\overline{\rho_{j}}\right\rangle\right)_{\text {equil }}=-\left(\rho_{f}-\rho_{g}\right) \frac{\left\langle\alpha_{v, \text { ave }}\right\rangle}{G_{\text {ave }}}\left(G_{i}-G_{j}\right)_{\text {equil }}
$$

In this equation, the equilibrium density distribution is posed as a function of the equilibrium mass flux distribution. It is a suitable approximation to use the actual mass flux distribution, $\left(G_{i}-G_{j}\right)$, in place of the equilibrium mass flux distribution, $\left(G_{i}-G_{j}\right)_{\text {equil }}$. The angle bracket operators in this equation represent an area-weighted average of the term in the brackets; these brackets can be omitted since the control volume discretization approach of $\mathrm{CTF}$ will lead to an averaged, uniform value for each term in each mesh cell. The "ave" subscripts mean that the term $(G$ and $\alpha)$ is the area-weighted average between the adjacent subchannels under consideration.

It is important to note that both the density and mass flux given here is that of the two-phase mixture. To get Lahey's equation in terms of the equilibrium void distribution, we begin by putting the two-phase mixture density in terms of void and saturation density and also expanding the average void and mass flux terms.

$$
\begin{aligned}
\left(\rho_{f}\left(1-\alpha_{v, i}\right)\right. & \left.+\rho_{g} \alpha_{v, i}-\rho_{f}\left(1-\alpha_{v, j}\right)-\rho_{g} \alpha_{v, j}\right)_{\text {equil }}= \\
& -\left(\rho_{f}-\rho_{g}\right) \frac{\alpha_{v, i} A_{i}+\alpha_{v, j} A_{j}}{\left(A_{i}+A_{j}\right)\left(G_{i} A_{i}+G_{j} A_{j}\right) /\left(A_{i}+A_{j}\right)}\left(G_{i}-G_{j}\right)
\end{aligned}
$$

At this point, we make a simplifying assumption that the adjacent subchannel areas, $A_{i}$ and $A_{j}$, are equal, thus eliminating them from the equation. Furthermore, it can be shown that the left-hand-side expression can be reduced as follows:

$$
-\left(\rho_{f}-\rho_{g}\right)\left(\alpha_{v, i}-\alpha_{v, j}\right)_{\text {equil }}=-\left(\rho_{f}-\rho_{g}\right) \frac{\alpha_{v, i}+\alpha_{v, j}}{G_{i}+G_{j}}\left(G_{i}-G_{j}\right)
$$

This can be further reduced to eliminate the difference between saturation densities. Additionally, a scaling factor, $K_{a}$, is added to account for geometry differences of different models. 


$$
\left(\alpha_{v, i}-\alpha_{v, j}\right)_{\text {equil }}=K_{a}\left(\alpha_{v, i}+\alpha_{v, j}\right) \frac{G_{i}-G_{j}}{G_{i}+G_{j}}
$$

The scaling factor is typically taken to be 1.4 [29], but it is made accessible via the CTF input deck so the user may adjust the value. We now substitute this derivation back into the void drift mass equations. In the process, we also substitute the saturation densities for actual space-dependent liquid and vapor density.

$$
\begin{aligned}
& W_{i j, v, V D}^{D^{\prime}}=V^{T}\left[K_{a}\left(\rho_{v, i} \alpha_{v, i}+\rho_{v, j} \alpha_{v, j}\right) \frac{G_{i}-G_{j}}{G_{i}+G_{j}}\right] \\
& W_{i j, l, V D}^{D^{\prime}}=-V^{T}\left[K_{a}\left(\rho_{l, i} \alpha_{v, i}+\rho_{l, j} \alpha_{v, j}\right) \frac{G_{i}-G_{j}}{G_{i}+G_{j}}\right]
\end{aligned}
$$

Note that the direction of the void drift term is opposite for vapor and liquid. The non-condensable gas mass void drift is formed by replacing vapor density with non-condensable gas density.

$$
W_{i j, \mathrm{gas}, V D}^{D^{\prime}}=V^{T}\left[K_{a}\left(\rho_{\mathrm{gas}, i} \alpha_{v, i}+\rho_{\mathrm{gas}, j} \alpha_{j}\right) \frac{G_{i}-G_{j}}{G_{i}+G_{j}}\right]
$$

This completes the derivation of the CTF void drift mass source terms. The void drift energy and momentum terms are derived similar to what was done for the turbulent-mixing terms. Equations 4.49 and 4.50 show the vapor and liquid energy mixing by void drift, respectively. No energy mixing takes place in the noncondensable gas or droplet fields by void drift.

$$
\begin{aligned}
& W_{i j, v, V D}^{H^{\prime}}=V^{T}\left[K_{a}\left(\rho_{v, i} \alpha_{v, i} h_{v, i}+\rho_{v, j} \alpha_{v, j} h_{v, j}\right) \frac{G_{i}-G_{j}}{G_{i}+G_{j}}\right] \\
& W_{i j, l, V D}^{H^{\prime}}=-V^{T}\left[K_{a}\left(\rho_{l, i} \alpha_{v, i} h_{l, i}+\rho_{l, j} \alpha_{v, j} h_{l, j}\right) \frac{G_{i}-G_{j}}{G_{i}+G_{j}}\right]
\end{aligned}
$$

Finally, Equations 4.51 and 4.52 show the vapor and liquid momentum mixing by void drift.

$$
\begin{aligned}
& W_{i j, v, V D}^{M^{\prime}}=V^{T}\left[K_{a}\left(\rho_{v, i} \alpha_{v, i} v_{v, i}+\rho_{v, j} \alpha_{v, j} v_{v, j}\right) \frac{G_{i}-G_{j}}{G_{i}+G_{j}}\right] \\
& W_{i j, l, V D}^{M^{\prime}}=-V^{T}\left[K_{a}\left(\rho_{l, i} \alpha_{v, i} v_{l, i}+\rho_{l, j} \alpha_{v, j} v_{l, j}\right) \frac{G_{i}-G_{j}}{G_{i}+G_{j}}\right]
\end{aligned}
$$

In CTF, mass flow rate is the solved term, so these equations are modified into the following form:

$$
W_{i j, v, V D}^{M^{\prime}}=V^{T}\left[K_{a}\left(\frac{\dot{m}_{v, i}}{A_{v, i}} \alpha_{v, i}+\frac{\dot{m}_{v, j}}{A_{v, j}} \alpha_{v, j} \frac{G_{i}-G_{j}}{G_{i}+G_{j}}\right]\right.
$$




$$
W_{i j, l, V D}^{M^{\prime}}=-V^{T}\left[K_{a}\left(\frac{\dot{m}_{l, i}}{A_{l, i}} \alpha_{v, i}+\frac{\dot{m}_{l, j}}{A_{l, j}} \alpha_{v, j} \frac{G_{i}-G_{j}}{G_{i}+G_{j}}\right]\right.
$$

Similar to the turbulent-mixing terms, the void drift source terms are not applied in all cases. If the flow regime is annular mist or mist, all void drift terms are left out of the CTF governing equations.

\subsubsection{Mixing Coefficient}

We now return to the definition of the mixing term, which has previously been condensed into the term, $V^{T}$.

$$
V^{T}=\epsilon \frac{s_{i j}}{z_{i j}^{T}}
$$

As previously noted, $s_{i j}$ is the gap width, which will be available in CTF from user input. The remainder of the term, $\epsilon / z_{i j}^{T}$ needs to be defined. To do this, we choose to start from the mixing parameter, $\beta$, which is defined as follows:

$$
\beta=\frac{\text { Transverse Mass Flux }}{\text { Axial Mass Flux }}
$$

Symbolically, this becomes:

$$
\beta=\frac{W_{i j}^{H^{\prime}} / s_{i j}}{\bar{G}}
$$

Here, $W_{i j}^{H^{\prime}}$ is the turbulent transverse mixing rate from Channel $i$ to $j$. It is divided by the gap width to convert it to mass flux. The $\bar{G}$ term is the area-weighted axial mass flux between the adjacent subchannels. The area-averaged axial mass flux is used because this is the definition of the mixing parameter; if we were to use the Channel $i$ axial mass flux instead, the mixing Stanton number would be formed. The transverse mixing rate will have both molecular and turbulent components. It is defined as shown below:

$$
W_{i j}^{H^{\prime}}=\mu s_{i j}\left[\frac{1}{z_{i j}^{L}}+\frac{\epsilon}{z_{i j}^{T} \nu}\right]
$$

This mixing term is actually defined for mixing of energy, but we make the assumption that energy, mass, and momentum are mixed at equal rates, which is a valid assumption for LWR conditions, where the turbulent Prandtl number will be close to unity. The first term in the brackets represents the molecular component of mixing. We neglect this term in CTF because the turbulent effects will dominate the mixing due to the relatively high mass flow rates found in LWRs. Making this simplification allows us to reduce this equation to the following: 
Substituting the definition of the lateral mixing mass flux back into Equation 4.57.

$$
\beta=\frac{\left(\epsilon / z_{i j}^{T}\right) \rho s_{i j} / s_{i j}}{\bar{G}}
$$

We can then reformulate this to solve for $\epsilon / z_{i j}^{T}$.

$$
\frac{\epsilon}{z_{i j}^{T}}=\frac{\beta \bar{G}}{\bar{\rho}}
$$

At this point, we also make the assumption that the density, $\rho$, that was used to define the kinematic viscosity, should be the area-average of the two adjacent subchannels under consideration. Now the mixing velocity, $\epsilon / z_{i j}^{T}$, can be fully defined if a value for $\beta$ is provided. This can either be provided by user input or calculated via the internal Rogers \& Rosehart correlation[31]. This will be discussed momentarily; however, in CTF, we will also need to know mixing term for two-phase flow, which will be higher than the single-phase value. This is done by introducing a multiplier term, $\Theta$, which is defined as follows:

$$
\Theta=\frac{\left(\epsilon / z_{i j}^{T}\right)_{\mathrm{TP}}}{\left(\epsilon / z_{i j}^{T}\right)_{\mathrm{SP}}}\left(1-\frac{\rho_{g}}{\rho_{l}}\right)
$$

We can multiply this by the single-phase term to get the two-phase value.

$$
\begin{gathered}
\left(\frac{\epsilon}{z_{i j}^{T}}\right)_{\mathrm{SP}} \Theta=\Theta \frac{\beta \bar{G}}{\rho_{i}} \\
\left(\frac{\epsilon}{z_{i j}^{T}}\right)_{\mathrm{TP}}=\Theta \frac{\beta \bar{G}}{\rho_{i}}
\end{gathered}
$$

We make note of the fact that both $\bar{G}$ and $\rho$ should be calculated with consideration that the fluid is actually a two-phase mixture. We may now proceed to providing defintions for $\Theta$ and $\beta$, which will fully close the turbulent mixing and void drift source terms.

The Beus correlation[32] provides definition for $\Theta$. The author demonstrated that the behavior of this multiplier is highly dependent on the flow regime. The multiplier was found to increase linearly with respect to flow quality up until the flow regime changed from slug to annular. As the flow quality continued to increase, the multiplier decreased in a hyperbolic fashion after that point. This behavior is shown graphically in Figure 4.2. Equations representing this behavior are shown below. 


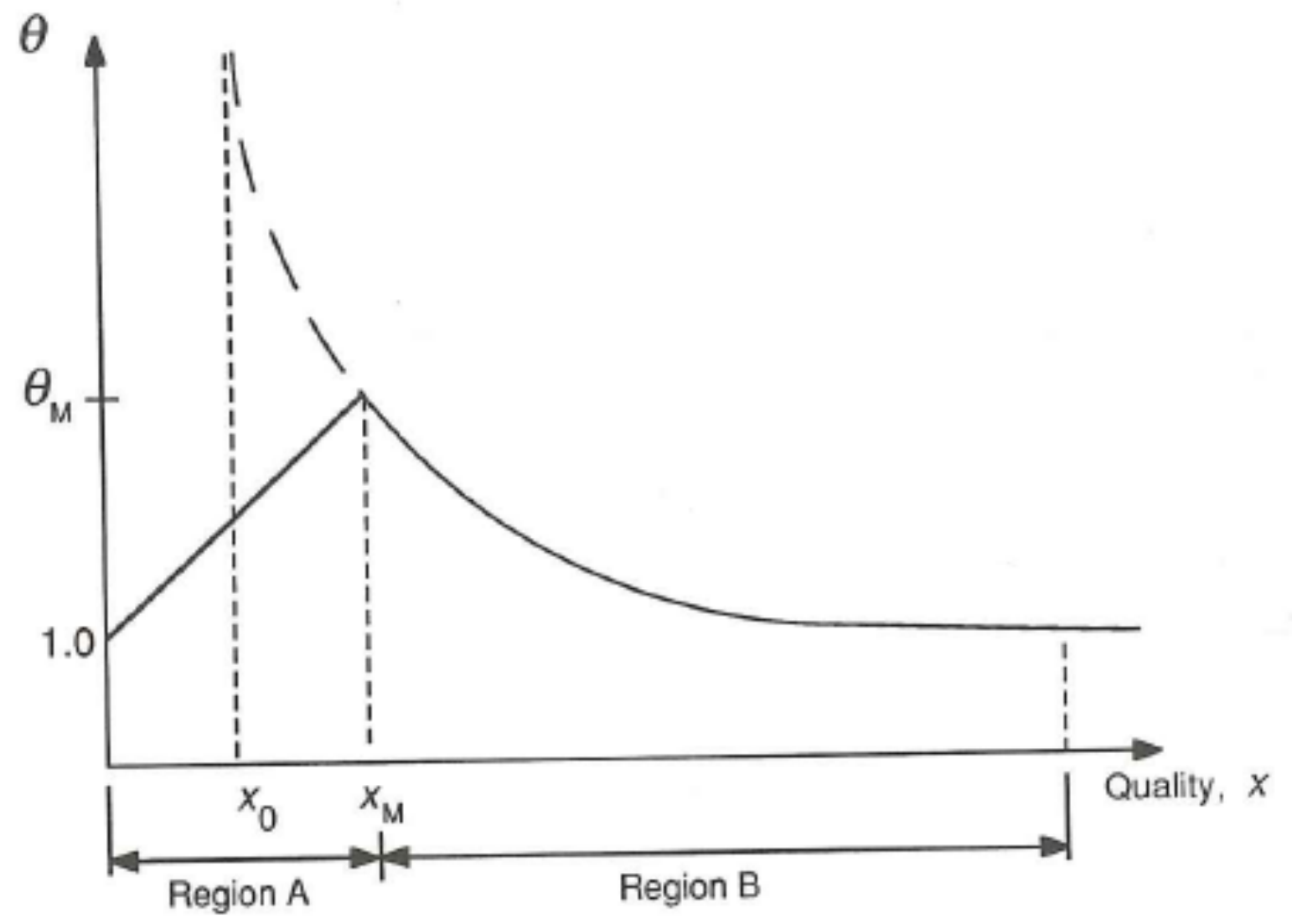

Figure 4.2: Behavior of the Beus two-phase mixing parameter

$$
\begin{aligned}
& \Theta=1+\left(\Theta_{M}-1\right)\left(\frac{x}{x_{M}}\right), \quad \text { if } x<x_{M} \\
& \Theta=1+\left(\Theta_{M}-1\right)\left(\frac{x_{M}-x_{0}}{x-x_{0}}\right), \quad \text { if } x>x_{M}
\end{aligned}
$$

Note that in Figure 4.2, the value of $\Theta$ for zero flow quality is 1.0. This will reduce the $\epsilon / z_{i j}^{T}$ term to its single-phase quantity. Therefore, it is suitable to always apply the $\Theta$ multiplier to the calculated single-phase mixing parameter, $\beta$.

The quality is denoted by $x$, the quality at the slug-annular transition point is denoted by $x_{M}$, and the value of the two-phase mixing coefficient at the transition point is denoted by $\Theta_{M}$. The equation capturing the hyperbolic nature of the two-phase mixing coefficient contains another term, $x_{0}$, which is simply the asymptote to which the hyperbola converges. The flow quality is defined as follows in CTF: 


$$
x=\frac{\dot{m}_{v}}{\dot{m}_{l}+\dot{m}_{e}+\dot{m}_{v}}
$$

The flow quality is calculated for the two adjacent sub-channels and then averaged to one value using area weighting. The quality at the slug-annular transition point is determined using Wallis's model [25].

$$
x_{M}=\frac{\frac{0.4\left[\rho_{f}\left(\rho_{f}-\rho_{g}\right) g D_{h}\right]^{1 / 2}}{G}+0.6}{\left(\frac{\rho_{f}}{\rho_{g}}\right)^{1 / 2}+0.6}
$$

The value of $\Theta_{M}$ was determined to be 5 by Faya [33]; however, CTF allows for the user to input their own value for $\Theta_{M}$. The value for the vertical asymptote, $x_{0}$, is given as a function of Reynolds number.

$$
x_{0}=x_{M} R e^{0.0417}
$$

One modification was made to the Beus correlation; the calculation of the asymptote value was modified to decrease the steep slope of the resulting hyperbola and improve numerical stability. The calculation of the asymptote instead becomes:

$$
x_{0}=0.75 x_{M} R e^{0.0417}
$$

The mixing parameter, $\beta$, can be specified using a flow-dependent correlation developed by Rogers \& Rosehart[31]. The correlation produces a single-phase mixing coefficient, as follows:

$$
\begin{aligned}
& \beta_{s p}=0.5 \lambda R e^{-0.1}\left[1+\left(\frac{D_{h, j}}{D_{h, i}}\right)^{1.5}\right] \frac{D_{h, i}}{D_{\text {rod }}}, \quad \text { if } D_{h, i}<D_{h, j} \\
& \beta_{s p}=0.5 \lambda R e^{-0.1}\left[1+\left(\frac{D_{h, i}}{D_{h, j}}\right)^{1.5}\right] \frac{D_{h, j}}{D_{\text {rod }}}, \quad \text { if } D_{h, j}<D_{h, i}
\end{aligned}
$$

The mixing coefficient is calculated for adjacent sub-channels, $i$ and $j$. The Reynolds number is calculated using the sub-channel with the smaller hydraulic diameter, $D_{h}$. If $R e$ is zero, the single-phase mixing coefficient, $\beta_{s p}$, is set to zero. The coefficient, $\lambda$, is calculated as follows:

$$
\lambda=0.0058\left(\frac{s_{i j}}{D_{\text {rod }}}\right)^{-1.46}
$$




\section{Micro-Mesh Cell Closure Models}

\subsection{Interfacial Drag Models}

The shear force present between different fields (vapor/droplets or vapor/liquid) is needed during the momentum equation solution. The interfacial drag force is calculated using the drag force equation.

$$
F_{D}=\frac{1}{2} \rho v^{2} C_{D} A
$$

In CTF, the interfacial drag force, $\tau_{i}$, is defined as a coefficient times the relative velocity between the phases. For interfacial drag between liquid and vapor in the axial direction:

$$
\tau_{i, v l}=k_{v l, x} U_{v l, x} \Delta X_{j}
$$

The momentum cell height, $\Delta X_{j}$, is required for calculating total drag force because the CTF source code defines the interfacial drag coefficient on a per unit length basis. This differs in form from the drag force equation in that the squared velocity was split apart into an explicit velocity term times an implicit velocity term. The implicit relative axial velocity between phases, $U_{v l, x}$, is solved during the momentum equation solution. The explicit velocity term, which is available using old time step information, is absorbed into the interfacial drag force coefficient, $k_{v l, x}$, along with the old time step density, interfacial area, and drag coefficient, $C_{D}$.

Both the drag coefficient, $C_{D}$, and the interfacial area, $A$, of Equation 5.1, will be highly dependent on the nature of the flow (i.e. flow regime) and, therefore, CTF includes different models for calculating these terms depending on the determined flow regime. Chapter 3 has already presented the manner by which CTF determines the flow regime and also how the interfacial area for that flow regime is calculated.

The following sections present the flow regime-specific calculation of the drag coefficient term needed for calculation of the interfacial drag force. Interfacial drag calculations are performed in the INTFR subroutine. Calculations are performed for the axial momentum cells and then transverse momentum cells. Consideration is made for both flow directions in discussing the flow regime-specific interfacial drag models that follow. 


\subsubsection{Small Bubble Regime}

The small bubble regime is characterized by uniformly dispersed vapor bubbles in a continuous liquid media. Being small, the geometry of the bubbles can be assumed as spherical. The small bubble regime is entirely composed of a uniform distribution of small bubbles; however, bubbles — small and large — can be found in other flow regimes, such as large bubble and churn-turbulent flow. The following interfacial drag models may also be applicable for the aforementioned flow regimes.

The interfacial drag coefficient is defined for the axial momentum cell as follows:

$$
k_{v l, x}=\frac{1}{2} \frac{C_{D} \rho_{l} \bar{U}_{v l} A_{p, s b}}{\Delta X_{j}}
$$

It is the projected area of the bubbles, $A_{p, s b}$, that is used in determining the drag force, and not the interfacial area. However, the interfacial area is related to the projected area for spherical geometry.

$$
A_{p, s b}=\frac{A_{i, s b}}{4}
$$

The interfacial drag coefficient calculation appears in the CTF code as:

$$
k_{v l, x}=\frac{1}{8} \frac{C_{D} \rho_{l}\left|U_{v}-U_{l}\right| A_{i, s b}}{\Delta X_{j}}
$$

It can be calculated for the lateral direction by using the lateral velocity instead of axial along with the appropriate terms for the transverse momentum cell.

$$
k_{v l, z}=\frac{1}{8} \frac{C_{D} \rho_{l}\left|W_{v}-W_{l}\right| A_{i, s b}}{\Delta Z_{k}}
$$

The interfacial area is calculated for the transverse momentum cell like for the axial momentum cells — the interfacial area was described by Equation 3.10 and is repeated here.

$$
A_{i, s b}=3 \alpha_{v} A_{x} \frac{\Delta X}{r_{b}}
$$

Substituting the gap cross-sectional area for axial momentum cell cross-sectional area produces the transverse momentum cell small bubble interfacial area. Substituting this result into Equation 5.6 leads to the form of the transverse drag force coefficient correlation used in CTF.

$$
k_{v l, z}=\frac{3}{8} \frac{C_{D b} \rho_{l}\left|w_{v l}\right|\left(1-\alpha_{v}\right) L_{g} \Delta Z_{k}}{r_{b}}
$$


As discussed by Ishii [34] and Ishii and Chawla [35], the bubble drag coefficient is dependent on the bubble Reynolds number as well as the regime (i.e. viscous regime, distorted particle regime, cap bubble regime, or Newton's regime). In the viscous region, bubbles behave like solid spheres. As Reynolds number increases and the flow enters the distorted particle region, the bubbles become distorted and exhibit irregular motion. As Reynolds increases further, still, the bubbles become spherical-cap shaped and the bubble drag coefficient becomes constant.

Viscous Region For the viscous region, Ishii [34] assumed that a particle in a multi-particle system has the same functional form as that of a single particle in an infinite media. The bubble drag coefficient is defined as:

$$
C_{D b}=\frac{24}{R e_{b}}\left(1.0+0.1 R e_{b}^{0.75}\right)
$$

The bubble Reynolds number is defined as:

$$
R e_{b}=\frac{2 r_{b} \rho_{l}\left|\bar{u}_{v l}\right|}{\mu_{m b}}
$$

Three terms are further defined. First, the velocity is the relative vector velocity between phases.

$$
\bar{u}_{v l}=\sqrt{\left(w_{v l}^{*}\right)^{2}+u_{v l}^{2}}
$$

Take note that $u_{v l}$ is defined for the axial momentum cell whereas $w_{v l}$ is defined for the transverse momentum cell which is staggered from the axial momentum cell location. Therefore, the transverse velocity at the axial location, $j$, must be calculated by averaging the transverse velocities above $(J+1)$ and below $(J)$.

$$
w_{v l}^{*}=\left|0.5\left(w_{v, J}+w_{v, J+1}\right)-0.5\left(w_{l, J}+w_{l, J+1}\right)\right|
$$

Secondly, the mixture viscosity must be appropriately calculated for the assumption that the particle in the multi-particle system behaves as a single particle in an infinite media. In a multi-particle system, a particle experiences greater resistance than in a single-particle system because the particle must deform not only the fluid, but also neighboring particles, too. Ishii [34] defined this as:

$$
\mu_{m b}=\mu_{l}\left(1-\alpha_{v}\right)^{-2.5 \frac{\left(\mu_{v}+0.4 \mu_{l}\right)}{\left(\mu_{v}+\mu_{l}\right)}}
$$

Third, and finally, the bubble radius is required for calculation of the bubble Reynolds number. The bubble radius was already defined in Chapter 3 by Equation 3.8. It is assumed to depend on a Weber number criterion, with the Weber number, We, equal to 10. It is repeated here for convenience. 


$$
r_{b}=\frac{0.5 W e_{b} \sigma g_{c}}{\rho_{l} \bar{u}_{v l}^{2}}
$$

The bubble radius is limited in the code to a maximum size of one half of the hydraulic diameter or $0.02 \mathrm{ft}$, whichever is smaller. When considering the transverse momentum cell, the bubble radius is calculated for the two channels that the gap connects to and the maximum bubble radius of the two channels, $i i$ and $j j$, is chosen for the transverse momentum cell. The transverse momentum cell bubble radius is further limited to be the minimum of the aforementioned bubble radius or half of the true gap width, which is the input gap width divided by the physical number of gaps, i.e.

$$
r_{b, t}=\min \left[r_{b, \max }, \frac{1}{2} \frac{L_{g}}{N_{g}}\right]
$$

The transverse momentum cell is prevented from being zero by setting it to either half the true gap width or $0.02 \mathrm{ft}$, whichever is smaller, should it be found to be zero.

Distorted Particle Region Like for the viscous region, it is assumed that a single particle behaves as a particle in an infinite media. It is also assumed that churn-turbulent flow will exist when in the distorted particle region. The bubble drag coefficient is given as:

$$
C_{D b}=\frac{\sqrt{2}}{3} N_{\mu} R e_{b}^{\prime}\left(1-\alpha_{v}\right)^{2}
$$

Where:

$$
N_{\mu}=\frac{\mu_{l}}{\left[\rho_{l} \sigma \sqrt{\frac{\sigma}{\left(\rho_{l}-\rho_{v}\right)}} g\right]^{\frac{1}{2}}}
$$

In the churn-turbulent regime, a particle tends to move in the wake caused by other particles, so the drift velocity is used in the calculation of bubble Reynolds number.

$$
R e_{b}^{\prime}=\frac{2 r_{b} \rho_{l}\left|\bar{u}_{v j}\right|}{\mu_{m}}
$$

The drift velocity, $\bar{u}_{v j}$, is defined as $\left(1-\alpha_{v}\right)\left|\bar{u}_{v l}\right|$. Again, the vector velocity is used as was defined for the viscous region. The mixture velocity is defined as follows:

$$
\mu_{m}=\frac{\mu_{l}}{\left(1-\alpha_{v}\right)}
$$


Cap Bubble Region The drag coefficient for the cap bubble region is simply:

$$
C_{D b}=\frac{8}{3}\left(1-\alpha_{v}\right)^{2}
$$

The bubble drag coefficient is calculated by each of the aforementioned means. The viscous regime drag coefficient is used if it is larger than the distorted particle regime drag coefficient. If the distorted particle regime drag coefficient is larger than the viscous regime drag coefficient, but smaller than the cap bubble regime drag coefficient, the distorted particle regime drag coefficient is used. Otherwise, if the distorted particle regime drag coefficient is larger than the other two drag coefficients, the cap bubble regime drag coefficient is used.

It should be noted that, in the source code, both the vector and the axial relative liquid-vapor velocities are limited to a maximum value of $1.4 \mathrm{ft} / \mathrm{s}$. This is to prevent the interfacial drag forces from growing to exceedingly large values, which is possible due to the large interfacial area in the small bubble regime.

\subsubsection{Slug Regime}

The slug regime is characterized by a mixture of small and large bubbles. The small bubble drag coefficient calculations that were presented in the previous section are used for the slug regime, too. The small bubbles are primarily in the viscous regime while large bubbles may be in Newton's regime. Bubbles in the slug regime move relative to the average volumetric flux. Therefore, the small bubble drag coefficient is modified by using the drift velocity instead of simply the relative phase velocity.

$$
\left.C_{D b}=C_{D b}\left(1-\alpha_{v}\right)^{2}\right)
$$

For spheres in the Newton regime, the drag coefficient becomes independent of the Reynolds number and, instead, becomes a constant of 0.45 . The large bubbles also move relative to the average volumetric flux, such that the large bubble drag coefficient is calculated as:

$$
C_{D b}=0.45\left(1-\alpha_{v}\right)^{2}
$$

The maximum of the viscous regime and Newton regime drag coefficients is taken for calculating the drag force coefficient. The axial drag force coefficient is calculated using Equation 5.3. The limits on relative velocity still apply for the large bubble regime. The drag for the slug regime is obtained by interpolating between the small and large bubble drag coefficients using the ratio of small bubble volume to total vapor volume, $F_{s b}$, as follows:

$$
k_{v l, x}=k_{v l, x, s b} F_{s b}+k_{v l, x, l b}\left(1-F_{s b}\right)
$$

$F_{s b}$ was defined in Section 3.2.3, but is repeated below. 


$$
F_{s b}=\frac{\alpha_{s b} \alpha_{l}}{\left(1-\alpha_{s b}\right) \alpha_{v}}
$$

\subsubsection{Dispersed Droplet Flow Regime}

The dispersed droplet flow regime is characterized by uniformly dispersed droplets in a continuous vapor media. The regime can exist in for normal or hot wall conditions. There may or may not be a continuous liquid field present.

For both hot and normal wall conditions, the droplet drag is calculated considering that the droplet behaves like a single particle in an infinite media, much like has been done for the small bubble regime. The model is based on the work of Ishii [34]. The drag coefficient is calculated as:

$$
k_{v e, x}=\frac{1}{8} C_{D d} \rho_{v}\left|u_{v e}\right| \frac{A_{i, d}}{\Delta X}
$$

$u_{v e}$ is the velocity difference between the vapor and droplet fields. The projected area of the droplets, $A_{i, d}$, is given by:

$$
A_{i, d}=\frac{3 \alpha_{e} A_{x} \Delta X}{r_{d}}
$$

For normal wall conditions, the droplet can exist in either the viscous or Newton regime. For the viscous regime, the droplet drag coefficient is calculated similar to how it was for small bubbles using Equation 5.8. The exception, is that the Reynolds number of the droplet is used instead of bubble Reynolds number. Droplet Reynolds number is calculated as follows:

$$
R e_{d}=\frac{D_{d} \rho_{v} \vec{u}_{v e}}{\mu_{m}}
$$

The droplet diameter is calculated using the droplet field interfacial area, as shown in Equation 3.32. The mixture viscosity, $\mu_{m}$, is calculated similar to that of mixture viscosity for small bubble flow.

$$
\mu_{m}=\mu_{v} \alpha_{v}-2.5 \frac{\mu_{l}+0.4 \mu_{v}}{\mu_{l}+\mu_{v}}
$$

For the case that the droplet is in the Newton regime, the drag coefficient is held constant at 0.45 . The maximum of the viscous regime and Newton regime drag coefficients is used for the normal wall mist regime droplet drag.

For the case of the hot wall regime, the viscous regime droplet drag coefficient correlation takes on a slightly different form. 


$$
C_{D d}=\frac{24}{R e_{d}}\left(1+0.15 R e_{d}^{0.687}\right)
$$

The droplet Reynolds number is also calculated differently, using the vapor viscosity in place of the mixture viscosity.

$$
R e_{d}=\frac{D_{d} \rho_{v} \vec{u}_{v e}}{\mu_{v}}
$$

The droplet drag coefficient for hot wall conditions, like for normal wall conditions, is calculated as the maximum of the Newton regime value (0.45) and the viscous regime value. For the transverse momentum cells, the drag coefficient is assumed to be characterized by the Newton regime and, therefore, the drag coefficient is 0.45 . The lateral droplet drag force coefficient is:

$$
k_{w e, z}=\frac{3}{8} * 0.45 \rho_{v}\left|w_{v}-w_{e}\right| \frac{\alpha_{e} L_{g} \Delta Z}{r_{d}}
$$

\subsubsection{Annular/Mist Regime}

The annular mist regime consists of a continuous liquid film that is spread across the solid surfaces in the cell and a suspension of uniformly distributed droplets throughout the vapor core. The drag force coefficient is calculated for both the liquid and droplet fields separately. The droplet field calculations are identical to those of the previous section.

The form of the film drag force coefficient is as follows:

$$
k_{v l, x}=\frac{1}{2} f_{i} \rho_{v}\left|u_{v}-u_{l}\right| \frac{A_{i x}}{\Delta X}
$$

The drag on the film is calculated using the interfacial area instead of the projected area, as was done for bubble and droplet fields. Furthermore, the friction factor is used in place of the drag coefficient. The interfacial area of the film can be determined considering a cylindrical geometry.

$$
\begin{aligned}
A_{i x} & =P_{w} \sqrt{\alpha_{v}+\alpha_{e}} \Delta X \\
& =4 \sqrt{\alpha_{v}+\alpha_{e}} A_{x} \frac{\Delta X}{D_{h}}
\end{aligned}
$$

For lateral flow, the physical number of gaps, $N_{g}$, is considered.

$$
A_{i z}=N_{g} \sqrt{\alpha_{v}+\alpha_{e}} \Delta X \Delta Z
$$


The interfacial friction factor is dependent on the nature of the film, be it stable or unstable. An unstable film will have large waves, which increase pressure drop and cause a higher friction factor. The stable film friction factor correlation was obtained from Wallis [25]. The unstable film friction factor was obtained from the work of Henstock and Hanratty [36].

The Wallis annular flow interfacial friction correlation is:

$$
f_{i w}=0.005\left(1+75\left(1-\alpha_{v}\right)\right)
$$

The Henstock and Hanratty correlation for unstable film is:

$$
f_{i u}=f_{s}\left(1+1400 F\left[1-\exp \left(\frac{-(1+1400 F)^{1.5}}{G_{a} 13.2 F}\right)\right]\right)
$$

The single-phase friction factor, $f_{s}$, is calculated using one quarter of the CTF rod friction factor model result for the vapor Reynolds number.

$$
f_{s}=\frac{1}{4}\left[0.204 R e_{v}^{-0.2}\right]
$$

The terms, $F$ and $G_{a}$, are defined as follows:

$$
\begin{gathered}
F=\frac{\left[\left(0.707 R e_{l}^{0.5}\right)^{2.5}+\left(0.0379 R e_{l}^{0.9}\right)^{2.5}\right]^{0.4}}{\operatorname{Re}_{v}^{0.9}} \frac{\mu_{l}}{\mu_{v}} \sqrt{\frac{\rho_{v}}{\rho_{l}}} \\
G_{a}=\frac{\rho_{l} g D_{h}}{\rho_{v} u_{v}^{2} f_{s}}
\end{gathered}
$$

The friction factor of the film is selected as the maximum of the unstable film friction factor and five times the stable film friction factor. The factor of 5 was obtained from observed differences between stable and unstable film pressure drop characteristics [37].

$$
f_{i f}=\max \left[5 f_{i w}, f_{i u}\right]
$$

For the lateral direction, only stable film is considered and the film friction factor is calculated as the number of physical gaps, $N_{g}$, times the Wallis correlation shown in Equation 5.32.

\subsubsection{Churn/Turbulent Flow Regime}

The churn/turbulent regime is considered to be a transition region between slug and annular-mist. The interfacial drag coefficient is interpolated between the interfacial drag coefficients calculated for the slug and annular-mist flow regime values. 


$$
k_{v l, x}=F_{c t} k_{v l, a m}+\left(1-F_{c t}\right) k_{v l, s}
$$

The interpolation factor is defined as:

$$
F_{c t}=\frac{\alpha_{v}-\alpha_{l b}}{\alpha_{\text {crit }}-\alpha_{l b}}
$$

The slug-to-churn/turbulent transition void fraction, $\alpha_{l b}$, is 0.5 . The churn/turbulent-to-annular/mist void fraction, $\alpha_{\text {crit }}$, is 0.8 .

The lateral direction interfacial drag for the churn/turbulent region is calculated in the same way as the axial direction.

\subsubsection{Inverted Annular Flow Regime}

The inverted annular flow regime is a hot wall regime. It is characterized by a liquid core surrounded by a vapor film. CTF only considers the inverted annular film regime for transverse momentum cells and not axial momentum cells. Actually, it is the only hot wall flow regime that is considered for transverse momentum cells - no other flow regimes are considered if a hot wall exists in a transverse momentum cell.

The interfacial drag is comprised of two components: drag on the interface between the vapor film and liquid core, and drag on bubbles inside of the vapor core. The film drag is calculated in the same manner as was done for annular/mist flow film, except that only stable film is considered using the Wallis correlation [25].

The Wallis correlation was shown in Equation 5.32 - note that the correlation is multiplied by a factor of 5 as it was for the annular/mist flow regime. Further, it is also multiplied by the physical number of gaps, $N_{g}$.

The bubble drag is calculated assuming that the bubbles are in the Newton regime as was done for the slug regime. The drag correlation is repeated below:

$$
C_{D b}=0.45\left(1-\alpha_{v}\right)^{2}
$$

\subsubsection{Falling Film Regime}

The falling film regime is the result of the core being re-flooded from the top. Above the quench front, a stable film forms on the solid surfaces. The film behaves similar to that of the annular/mist flow regime except that the film is always assumed to be stable. Therefore, only the Wallis [25] friction factor correlation is used for determining the interfacial friction coefficient, repeated here:

$$
f_{i w}=0.005\left(1+75\left(1-\alpha_{v}\right)\right)
$$


The friction coefficient is not multiplied by a factor of 5 as it was for the annular/mist flow regime since the film is assumed stable. The falling film flow regime is not considered for transverse momentum cells. The interfacial drag force coefficient is calculated as:

$$
k_{v l}=f_{i w} \rho_{v}\left|u_{v}-u_{l}\right| \frac{A_{i}}{\Delta X}
$$

The interfacial area is used, as was done for the annular/mist regime. In fact, the interfacial area calculation is the same, which can be found in Chapter 3. To account for film dryout, a ramp is multiplied by the interfacial drag force coefficient.

$$
R=\max \left[0.001, \min \left(1.0, \frac{\alpha_{l}}{0.005}\right)\right]
$$

Dispersed droplets can also exist in the vapor core. The interfacial drag is calculated as was discussed in Section 5.1.3 for the hot wall case.

\subsubsection{Top Deluge Flow Regime}

The deluge regime consists of large liquid chunks falling down through the channels. It can be a result of upper plenum injection during accident conditions. It exists for hot wall conditions and void fractions less than 0.8 .

The interfacial drag is modeled considering droplets in the viscous or Newton regime. The viscous regime drag coefficient is calculated similarly as was done for the small bubble regime in Equation 5.8; however, with the vapor phase Reynolds number used instead of bubble Reynolds number.

$$
C_{D}=\frac{24}{R e_{v}}\left[1.0+0.1 R e_{v}^{0.75}\right]
$$

The Reynolds number for the vapor phase is calculated as:

$$
R e_{v}=\frac{G_{v} D_{h}}{\mu_{v}}
$$

Where,

$$
G_{v}=\frac{\dot{m}_{v} \alpha_{v}}{A_{x}}
$$

The Newton regime drag coefficient is a constant of 0.45 . The maximum of the viscous regime and Newton regime drag coefficients is used in calculating the interfacial drag force coefficient for the top deluge regime. The interfacial drag force coefficient is calculated as: 


$$
k_{v l}=\frac{1}{2} C_{D} \rho_{v}\left|u_{v}-u_{l}\right| \frac{A_{i}}{4 \Delta X}
$$

The interfacial area is converted to projected area in this case and, since the liquid void is high, the droplets are treated as continuous liquid and the liquid velocity is used instead of the droplet velocity.

For vapor void below 0.6 , the top deluge interfacial drag dominates; however, as vapor void increases above that, the falling film drag effects begin to make an impact. A linear ramp is used between 0.6 and 0.8 for the top deluge and falling film drag force coefficients.

\subsection{Interfacial Heat Transfer Models}

The interfacial heat transfer coefficients are used for determination of the implicit vapor generation rate (or condensation rate) and, in turn, the transfer of enthalpy from one phase to another. The interfacial heat transfer can be thought of as going from the phase to the interface or vice versa. Interfacial heat transfer coefficients are calculated for both field interfaces (i.e. droplet/vapor and liquid/vapor) and for four different possible scenarios:

\section{Subcooled Liquid}

2. Superheated Liquid

3. Subcooled Vapor

4. Superheated Vapor

For example, if a subcooled liquid is present, regardless of what the vapor temperature is, a subcooled liquid heat transfer coefficient will be calculated. Heat transfer is from the interface to the liquid and condensation is the result (the magnitude of the condensation is dependent on the difference between interface and subcooled liquid temperature). All four interfacial heat transfer coefficients are calculated for both field interfaces in INTFR. CTF then later checks, in XSCHEM, whether the fluid is subcooled or superheated - appropriate terms are then zeroed.

The resulting interfacial heat transfer coefficients for both field interfaces are combined (i.e. subcooled liquid/ vapor interfacial heat transfer is added to subcooled droplet/vapor interfacial heat transfer, superheated liquid/vapor interfacial heat transfer is added to superheated droplet/vapor interfacial heat transfer, etc.).

The interfacial heat transfer terms are used to calculate the rate of phase change in the continuity/energy conservation equation solution in XSCHEM. The calculated mass transfer rate is then also used in the momentum equation solution to calculate momentum transfer by phase change in the next time step. Since the terms are defined for the momentum equation, but are used in the continuity/energy equation solution, adjacent momentum cell values are averaged to find the continuity mesh cell value located between the two. The mass transfer rate, $\Gamma^{\prime \prime \prime}$, is calculated as shown in Equation 5.46. 


$$
\begin{aligned}
\Gamma_{\text {evap }, \text { shl }} & =\frac{h_{\text {int }, \text { shl }}}{\left(h_{g}-h_{f}\right) C_{p, l}}\left|h_{l}-h_{f}\right| \\
\Gamma_{\text {evap }, \text { shv }} & =\frac{h_{\text {int }, \text { shv }}}{\left(h_{g}-h_{f}\right) C_{p, v}}\left|h_{v}-h_{g}\right| \\
\Gamma_{\text {cond,scl }} & =\frac{h_{\text {int }, \mathrm{scl}}}{\left(h_{g}-h_{f}\right) C_{p, l}}\left|h_{l}-h_{f}\right| \\
\Gamma_{\text {cond,scv }} & =\frac{h_{\text {int }, \text { scv }}}{\left(h_{g}-h_{f}\right) C_{p, l}}\left|h_{v}-h_{g}\right|
\end{aligned}
$$

Subcooled liquids and vapors lead to condensation and superheated fluids lead to evaporation. The $h_{f}$ and $h_{g}$ terms are the saturation enthalpies of liquid and vapor, the $h_{l}$ and $h_{v}$ terms are the liquid and vapor enthalpies (which may be less than or greater than the saturation enthalpies depending on if the fluid is superheated or subcooled), and $C_{p, l}$ and $C_{p, v}$ are the specific heats of liquid and vapor.

Two out of the four terms in Equation 5.46 will be zero because a fluid cannot be both superheated and subcooled. The mass transfer rates are additionally multiplied by ramps for code stability. The total mass transfer is simply found by subtracting condensation terms from evaporation terms, as shown in Equation 5.47 .

$$
\Gamma_{\text {net }}=\left[\Gamma_{\text {evap }, \mathrm{shl}}+\Gamma_{\text {evap }, \text { shv }}\right]-\left[\Gamma_{\text {cond,scl }}+\Gamma_{\text {cond,scv }}\right]
$$

The total phase change contributes to the continuity equation residuals. When mass changes phases, it also takes phase enthalpy along with it (i.e. evaporation cools a superheated liquid, while condensation will warm a subcooled liquid). Energy exchange by phase mass transfer is calculated as shown in Equation 5.48.

$$
\Gamma_{\text {net }} H=\left[\Gamma_{\text {evap }, \text { shl }}-\Gamma_{\text {cond,scl }}\right] h_{f}+\left[\Gamma_{\text {evap }, \text { shv }}-\Gamma_{\text {cond,scv }}\right] h_{g}
$$

The interfacial heat transfer coefficients $-h_{i, \mathrm{shl}}, h_{i, \mathrm{scl}}, h_{i, \mathrm{shv}}$, and $h_{i, \mathrm{scv}}-$ will be dependent on the flow regime as will be the interfacial area for heat transfer. Models for calculating the interfacial heat transfer coefficients are discussed in the following sections. The interfacial area for heat transfer was previously discussed in Chapter 3. The INTFR subroutine calculates both interfacial heat transfer coefficients and interfacial heat transfer area and returns the product of the two to the XSCHEM subroutine.

\subsubsection{Small Bubble Flow Regime}

Superheated Vapor The interfacial heat transfer coefficient for a superheated vapor is assumed to be a constant in CTF.

$$
h_{i, \mathrm{shv}}=2.78 \mathrm{BTU} / \mathrm{ft}^{2} \cdot \mathrm{sec}^{\circ}{ }^{\circ} \mathrm{F} A_{i}
$$


Superheated Liquid The interfacial heat transfer coefficient for a superheated liquid is calculated considering two effects; conduction and convection. Convection is calculated using a correlation by Rowe [38].

$$
h_{i, \mathrm{shl}, \mathrm{conv}}=\frac{k_{f}}{D_{b}}\left(2.0+0.74 \operatorname{Re}_{b}^{1 / 2} \operatorname{Pr}_{l}^{1 / 3}\right)
$$

The bubble Reynolds number is found as follows:

$$
R e_{b}=\frac{\rho_{l} \vec{u}_{r} D_{b}}{\mu_{l}}
$$

The velocity utilized is the vector sum of axial and transverse components of relative (i.e. relative between vapor and liquid) velocities.

The conduction component of interfacial heat transfer is defined using the Jakob number:

$$
\begin{aligned}
h_{i, \mathrm{shl}, \mathrm{cond}} & =\frac{k_{f}}{D_{b}} J a, \quad \text { where } \\
J a & =\frac{\rho_{l}\left(h_{l}-h_{f}\right)}{\rho_{v} h_{\mathrm{fg}}}
\end{aligned}
$$

The maximum of the convective and conduction heat transfer coefficients is used for the interfacial heat transfer of a superheated liquid. The effect of nucleation at heated surfaces is added to this term.

$$
h_{i, \mathrm{shl}}=h_{i, \mathrm{shl}} A_{i}+h_{\mathrm{nb}} A_{p w}
$$

Where:

$$
h_{\mathrm{nb}}=\frac{k_{f}}{D_{b}} \max \left(8.0,0.023\left(\frac{\rho_{l} \vec{u}_{l} D_{b}}{\mu_{m}}\right)^{0.8} \operatorname{Pr}_{l}^{0.4}\right)
$$

The mixture viscosity was defined by Equation 5.12. The heat transfer area for nucleate boiling is simply the heated perimeter times cell height.

Subcooled Vapor The subcooled vapor interfacial heat transfer coefficient is set to a constant value, as was done for the superheated vapor.

$$
h_{i, \mathrm{scv}}=2.78 \mathrm{BTU} / \mathrm{ft}^{2} \cdot \mathrm{sec}^{\circ}{ }^{\circ} \mathrm{F} A_{i}
$$


Subcooled Liquid The subcooled liquid interfacial heat transfer coefficient is calculated as follows [39]:

$$
h_{i, \mathrm{scl}}=1.1284 \sqrt{\frac{k_{f}}{D_{b}} \vec{u}_{v l} \rho_{l} C_{p, l}} A_{i}
$$

\subsubsection{Slug Regime}

The slug regime is a combination of small and large bubbles. The small bubble interfacial heat transfer coefficient models were shown in the previous section. The following models are for large bubbles. The previously defined ramping factor, $F_{s b}$, (see Section 3.2.3) is used to perform a linear interpolation between interfacial heat transfer coefficients for each of superheated liquid, superheated vapor, subcooled liquid, and subcooled vapor. The calculation of $F_{s b}$ is repeated:

$$
F_{s b}=\frac{\alpha_{s b} \alpha_{l}}{\left(1-\alpha_{s b}\right) \alpha_{v}}
$$

The slug regime interfacial heat transfer coefficients are calculated as follows:

$$
\begin{aligned}
& h_{i, \mathrm{shl}}=F_{s b} h_{i, \mathrm{shl}, s b}+\left(1-F_{s b}\right) h_{i, \mathrm{shl}, l b} \\
& h_{i, \mathrm{shv}}=F_{s b} h_{i, \mathrm{shv}, s b}+\left(1-F_{s b}\right) h_{i, \mathrm{shv}, l b} \\
& h_{i, \mathrm{scl}}=F_{s b} h_{i, \mathrm{scl}, s b}+\left(1-F_{s b}\right) h_{i, \mathrm{scl}, l b} \\
& h_{i, \mathrm{scv}}=h_{i, \mathrm{scv}, l b}
\end{aligned}
$$

Large Bubble Superheated Liquid A constant value is assumed for the superheated liquid interfacial heat transfer coefficient.

$$
h_{i, \mathrm{shl}}=278.0^{\mathrm{BTU}} / \mathrm{ft}^{2} \cdot \mathrm{sec}^{\circ}{ }^{\circ} \mathrm{F}
$$

Large Bubble Superheated Vapor The interfacial heat transfer coefficient for superheated vapor is calculated using the correlation of Lee and Ryley[40].

$$
h_{i, \mathrm{shv}}=\frac{k_{l}}{D_{h}}\left(2.0+0.74 R e^{0.5} \operatorname{Pr}^{0.33}\right) A_{i}
$$

Large Bubble Subcooled Liquid The same correlation that was used for the small bubble regime is used for the large bubble regime in calculating the subcooled liquid interfacial heat transfer coefficient. 


$$
h_{i, \mathrm{scl}}=1.1284 \sqrt{\frac{k_{f}}{D_{b}} \vec{u}_{v l} \rho_{l} C_{p, l}} A_{i}
$$

Large Bubble Subcooled Vapor The interfacial heat transfer coefficient for subcooled vapor is calculated using the Lee and Ryley correlation (see Equation 5.59 that was used for the superheated vapor interfacial heat transfer coefficient.

\subsubsection{Dispersed Droplet Flow Regime}

Superheated Liquid In the case of normal wall conditions, the superheated liquid interfacial heat transfer coefficient is set equal to the value calculated for the subcooled liquid heat transfer coefficient. For the case of hot wall conditions, the interfacial heat transfer coefficient is set to a constant value.

$$
h_{i, \operatorname{shl}, h w}=278 \mathrm{BTU} / \mathrm{ft}^{2} \cdot \sec \cdot{ }^{\circ} \mathrm{F} A_{i, d}
$$

Superheated Vapor For normal wall conditions, a correlation in the same form as the Lee-Ryley correlation (see Equation 5.59) is used to calculate the droplet interfacial heat transfer coefficient. This correlation is by Frössling[41] and has a change in the coefficient leading Re. Furthermore, the correlation is divided by a modification factor defined by Yuen and Chen[42].

$$
h_{i, \mathrm{shv}}=\frac{\frac{k_{v}}{D_{d}}\left(2.0+0.55 \operatorname{Re}^{0.5} \operatorname{Pr}^{0.33}\right) A_{i}}{F_{y c}}
$$

Note that the vapor conductivity is used and it is evaluated at the film temperature, which is the average of the heated surface temperature and the bulk vapor temperature. The Yuen and Chen factor is defined below:

$$
F_{y c}=1.0+0.5 \frac{h_{v}-h_{g}}{h_{f g}}
$$

Subcooled Liquid The same correlation is used for hot and normal wall conditions. This value is adopted for the superheated liquid interfacial heat transfer coefficient in normal wall conditions. This correlation is derived by muliplying the Anderson correlation [43] by 2.7 .

$$
h_{i, \mathrm{scl}}=8.88 \frac{k_{f}}{R_{d}} A_{i, d}
$$


Subcooled Vapor In the case of normal wall conditions, the subcooled vapor interfacial heat transfer coefficient takes on the value of the normal wall superheated vapor interfacial heat transfer coefficient, which was obtained using the modified Lee-Ryley correlation (by Frössling). For hot wall conditions, a constant value is used.

$$
h_{i, \mathrm{scv}, h w}=2.78 \mathrm{BTU} / \mathrm{ft}^{2} \cdot \mathrm{sec} \cdot{ }^{\circ} \mathrm{F}
$$

\subsubsection{Annular/Mist Flow Regime}

The annular/mist regime consists of a liquid film and a suspended droplet field. The interfacial heat transfer coefficients for the droplet field were discussed in the previous section. The normal wall models apply for the annular/mist flow regime. The droplet and film interfacial heat transfer coefficients are simply added together for each of the four cases (superheated liquid, superheated vapor, subcooled liquid, and subcooled vapor).

Superheated Liquid There are three possible expressions for the superheated liquid. The first evaluation is between a constant value of $278 \mathrm{BTU} / \mathrm{ft}^{2} \cdot \mathrm{sec} \cdot{ }^{\circ} \mathrm{F}$ or the conduction through the film. The minimum value of these two expressions is taken. The third is the Colburn analogy[44] for heat transfer from a film using the Hughmark $[45,46]$ correlation for the film friction factor. The maximum of the Colburn analogy and the result of the first evaluation is taken. This is denoted mathematically as follows.

$$
h_{i, \mathrm{shl}}=\max \left[h_{i, \mathrm{shl}, \mathrm{colburn}}, \min \left(278 \mathrm{BTU} / \mathrm{ft}^{2} \cdot \mathrm{sec} \cdot{ }^{\circ} \mathrm{F}, h_{i, \mathrm{shl}, \mathrm{cond}}\right)\right]
$$

The conduction heat transfer term is defined using the saturated liquid conductivity, $k_{f}$, and the film thickness, $\delta_{l}$.

$$
h_{i, \mathrm{shl}, \mathrm{cond}}=\frac{2 k_{f}}{\delta_{l}} P_{w} \Delta X
$$

The Colburn analogy[44] is:

$$
h_{i, \mathrm{shl}, \mathrm{colburn}}=f_{\mathrm{HM}} \rho_{l} C_{p, l}\left|u_{v l}\right| \operatorname{Pr}^{-2 / 3} A_{i, f} f_{a i}
$$

The Hughmark[46] friction factor correlation is defined:

$$
\begin{aligned}
& f_{\mathrm{HM}}=3.850 R e^{-2 / 3}, \quad \text { if } R e_{l}<1000 \\
& f_{\mathrm{HM}}=0.5402 R e^{-0.38}, \quad \text { if } R e_{l}>=1000
\end{aligned}
$$

In CTF, however, the leading coefficient for the case of $R e_{l}<1000$ is cut in half. 


$$
f_{\mathrm{HM}}=1.925 R e^{-2 / 3}, \quad \text { if } R e_{l}<1000
$$

Returning to Equation 5.67, the result is multiplied by the interfacial area of the film, $A_{i, f}$, as well as a multiplication factor, which is designed to ramp the interfacial heat transfer to zero as the liquid film drys out. The factor begins to decrease from 1.0 as the liquid void drops below 0.01 .

$$
f_{a i}=\max \left[0.0, \min \left(1.0,111.1\left(\alpha_{l}-0.001\right)\right)\right]
$$

Superheated Vapor The Colburn analogy[44] is used for calculating the superheated vapor interfacial heat transfer coefficient. However, different from the case of superheated liquid, the friction factor is determined using the method of Wallis[25] (stable film) or Hanratty[36] (unstable film). The vapor density is used, the vector relative velocity is used, the vapor specific heat capacity at the film temperature is used, and no multiplication factor is necessary for the case of dryout.

$$
h_{i, \mathrm{shv}}=f_{i} \rho_{v} C_{p, \text { film }} \vec{u}_{v l} \operatorname{Pr}^{-2 / 3} A_{i, \text { film }}
$$

The Wallis and Hanratty correlations were previously shown in Equations 5.32 and 5.33, respectively. The film temperature is defined as the average of the heated surface wall temperature and the bulk vapor temperature.

Subcooled Liquid The subcooled liquid interfacial heat transfer coefficient is calculated using he same for of the Colburn analogy[44] that was utilized for superheated liquid.

Subcooled Vapor The subcooled vapor interfacial heat transfer coefficient is calculated using the form of the Colburn analogy[44] that was used for the case of superheated vapor.

\subsubsection{Churn-Turbulent Flow Regime}

The churn-turbulent flow regime is a combination of the annular/mist and slug flow regimes. An interpolation factor was defined previously in Equation 5.39, which is repeated below.

$$
F_{c t}=\frac{\alpha_{v}-\alpha_{l b}}{\alpha_{\text {crit }}-\alpha_{l b}}
$$

The interfacial heat transfer coefficients calculated for the slug and annular/mist flow regimes are used to calculate the churn-turbulent interfacial heat transfer coefficients. 


$$
\begin{aligned}
& h_{i, \mathrm{shv}}=F_{c t} h_{i, \mathrm{shv}, \mathrm{AM}}+\left(1-F_{c t}\right) h_{i, \mathrm{shv}, \mathrm{S}} \\
& h_{i, \mathrm{shl}}=F_{c t} h_{i, \mathrm{shl}, \mathrm{AM}}+\left(1-F_{c t}\right) h_{i, \mathrm{shl}, \mathrm{S}} \\
& h_{i, \mathrm{scv}}=F_{c t} h_{i, \mathrm{scv}, \mathrm{AM}}+\left(1-F_{c t}\right) h_{i, \mathrm{scv}, \mathrm{S}} \\
& h_{i, \mathrm{scl}}=F_{c t} h_{i, \mathrm{scl}, \mathrm{AM}}+\left(1-F_{c t}\right) h_{i, \mathrm{scl}, \mathrm{S}}
\end{aligned}
$$

\subsubsection{Falling Film and Deludge Flow Regimes}

The interfacial heat transfer coefficients of the falling film and deludge flow regimes are calculated in the same way. Interfacial heat transfer coefficients are calculated for the film and droplet fields and then added together as was done for the annular/mist regime. However, in this case the hot wall models are used for the dispersed droplet regime. The film interfacial heat transfer coefficients are discussed below.

Superheated Liquid The interfacial heat transfer coefficient for superheated liquid is a constant value.

$$
h_{i, \mathrm{shl}}=27.8 \mathrm{BTU} / \mathrm{ft}^{2} \cdot \mathrm{sec} \cdot{ }^{\circ} \mathrm{F}
$$

Superheated Vapor The superheated vapor interfacial heat transfer coefficient is calculated by the same approach as was done for the droplet superheated vapor interfacial heat transfer coefficient in the case of hot wall conditions. The modified Lee-Ryley correlation is repeated here:

$$
h_{i, \mathrm{shv}}=\frac{\frac{k_{v}}{D_{h}}\left(2.0+0.55 R e_{v}^{0.5} P r^{0.33}\right) A_{i}}{F_{y c}}
$$

Where the Yuen and Chen factor, $F_{y c}$, is given as follows:

$$
F_{y c}=1.0+0.5 \frac{h_{v}-h_{g}}{h_{\mathrm{fg}}}
$$

Additionally, there is consideration for the effect of a housing quench front boundary. If a housing quench front boundary exists and $R e<25200$, the Nusselt number is calculated as follows:

$$
N u_{q h}=\max \left[10,0.0797 \operatorname{Re}_{v}^{0.6774} \operatorname{Pr}_{v}^{0.333}\right]
$$

For $R e>25200$ :

$$
N u_{q h}=0.023 \operatorname{Re}_{v}^{0.8} \operatorname{Pr}_{v}^{0.4}
$$


The single phase vapor heat transfer coefficient is then:

$$
h_{\mathrm{spv}}=\frac{k_{v}}{D_{h}} N u_{q h}
$$

The interfacial heat transfer coefficient for superheated vapor is modified by adding the contribution of the wet film on the quenched housing.

$$
h_{i, \mathrm{shv}}=h_{i, \mathrm{shv}}+h_{\mathrm{spv}} P_{w, c} \Delta X \min \left[1,0.5+X_{q}\right]
$$

The wetted perimeter of the housing (unheated conductor) is given by $P_{w, c}$. The fraction of the conductor which is quenched, $X_{q}$, is considered in the last term.

Subcooled Liquid For subcooled liquid, the interfacial heat transfer is given by:

$$
h_{i, \mathrm{scl}}=17.77 \frac{k_{f}}{D_{h}} A_{i}
$$

Subcooled Vapor The subcooled vapor interfacial heat transfer coefficient is assumed to be a constant value.

$$
h_{i, \mathrm{scv}}=2.78 \mathrm{BTU} / \mathrm{ft}^{2} \cdot \mathrm{sec}^{\circ}{ }^{\circ} \mathrm{F}
$$

\subsubsection{Inverted Annular Flow Regime}

The inverted annular film flow regime is only considered for transverse momentum cells. Interfacial heat transfer coefficients are not calculated for transverse momentum cells - only axial momentum cells. Therefore, no interfacial heat transfer models are discussed here.

\subsection{Entrainment and De-Entrainment Models}

\subsubsection{Introduction}

The physical processes of entrainment and de-entrainment must be modeled to account for interaction between the continuous liquid and droplet fields. Four scenarios are considered in CTF when modeling droplet sources:

1. Entrainment from liquid film 
2. Entrainment during bottom reflood

3. Entrainment during top down reflood

4. Spacer grid droplet breakup

The first three cases involve mass leaving the liquid field and entering the droplet field, but by different processes. The fourth case doesn't add droplets by entrainment from the liquid field, but instead accounts for the increase in droplets and droplet surface area due to droplets breaking up on spacer grids — an effect that is important to core cooling during accident where core dryout occurs.

Mass can also move from the droplet field to liquid field by the process of de-entrainment. Four scenarios are considered when modeling de-entrainment:

1. De-entrainment in film flow

2. De-entrainment in crossflow

3. De-entrainment due to area changes

4. De-entrainment at solid surfaces and liquid pools

\subsubsection{Liquid Film Entrainment/De-entrainment}

Entrainment Two cases are considered when calculating the entrainment rate in the annular/mist flow regime: entrainment in co-current flow and entrainment in counter-current flow. The phenomenon responsible for entrainment from film is the same for the two cases. Waves on the film surface will grow as a result of hydrodynamic and surface tension forces. When the pressure differential over the wave exceeds the surface tension due to the amplitude of the wave growing large, the wave will break and liquid will be entrained. The wave behavior, and entrainment rate, will differ, however, depending on if the flow is co-current - low amplitude roll waves with droplets being sheared off of the wave crest [45] — or counter-current — abrupt, large-amplitude waves.

CTF will check if the flow is counter-current by checking for negative liquid mass flow rate and positive vapor mass flow rate in the momentum cell below the current one. If in counter-current flow, the maximum of the co-current and counter current entrainment rates is taken. For either case, the entrainment rate is limited to the smaller of two values: the calculated entrainment rate, or a function of the maximum flow in the cell:

$$
S_{E}=\min \left[S_{E},\left(F_{s e}+0.05 \alpha_{l} \rho_{l} A_{x}\right)\right]
$$

The maximum flow is calculated by considering the total liquid lateral flow — all gap liquid flow into the cell plus any boundary condition injection flows - and the axial liquid flow rate.

\footnotetext{
${ }^{1}$ This section presents the entrainment/de-entrainment models used currently in COBRA-TF, but improvements have been made to these models in the past, which is documented in Ha, Jeong, and Sim[47].
} 
Counter-Current Flow For counter-current flow, it is assumed that anything in excess of the critical liquid flow rate is sheared off into the droplet field.

$$
S_{E, \text { counter }}=\dot{m}_{l}-\dot{m}_{l, \text { crit }}
$$

Expanding,

$$
S_{E, \text { coutner }}=\left(\alpha_{l}-\alpha_{l, \text { crit }}\right) \rho_{l}\left|U_{l}\right| A_{x}
$$

The critical liquid void fraction is calculated from the critical vapor void fraction:

$$
\alpha_{l, \mathrm{crit}}=1-\alpha_{\text {crit }}
$$

The critical vapor void fraction is determined from a force balance between opposing forces acting on the wave - the pressure gradient over the wave crest and the surface tension.

$$
\alpha_{\text {crit }}=1-2 \frac{\sigma}{\rho_{v} \vec{U}_{v l}^{2} D_{h}}
$$

A modified relative velocity is used in calculating the critical void. The maximum of the relative vapor-liquid vector velocity and a modified axial relative velocity is used.

$$
\vec{U}_{v l}=\max \left[\vec{U}_{v l},\left(u_{v, j-1}-u_{l, j-1}\right) \frac{1-\alpha_{l}}{1-2.5 \alpha_{l}}\right]
$$

Co-Current Flow Whalley, Hewitt, and Hutchinson[48] correlated entrainment data for air-water mixtures as a function of interfacial shear stress, $\tau_{i}$, surface tension, $\sigma$, and a roughness factor acting as the length scale, $k_{s}$, using the dimensionless group

$$
S_{k}=k_{s} \frac{\tau_{i}}{\sigma}
$$

This was modified by Wurtz[49] by multiplying by the dimensionless velocity in order to correlate data for both air-water and steam-water mixtures, according to

$$
S_{w}=\frac{k_{s} \tau_{i} \vec{U}_{v} \mu_{l}}{\sigma^{2}}
$$

This form was later used by Paleev and Filippovich[50] to correlate air-water data. Wurtz used this dimensionless group to obtain the following relationship which is used in CTF for co-current entrainment rate: 


$$
S_{E}=\left[0.41 \mathrm{lbm} \mathrm{ft}{ }^{-2} \mathrm{~s}\right]\left[\frac{k_{s} \tau_{i} \vec{U}_{v} \mu_{l}}{\sigma^{2}}\right] P_{w} \Delta X
$$

The roughness parameter is given by

$$
k_{s}=0.57 \delta+6623 \mathrm{ft}^{-1} \delta^{2}-3.56 \times 10^{6} \mathrm{ft}^{-2} \delta^{3}+1.5767 \times 10^{9} \mathrm{ft}^{-3} \delta^{4} .
$$

The interfacial shear is calculating using the interfacial friction factor for the film, $f_{i}$, which was discussed in Section 5.1.4:

$$
\tau_{i}=f_{i} \rho_{v} \vec{U}_{v l}^{2}
$$

De-Entrainment De-entrainment in film flow is due to the random turbulent motions of the droplets that cause some of them to impact the film and be absorbed. The de-entrainment rate is calculated using the correlation of Cousins[51], which uses a droplet concentration gradient diffusion model. The form of the correlation is

$$
S_{D E}=k_{0} C P_{w} \Delta X
$$

The droplet concentration, $C\left[1 \mathrm{bm} \mathrm{ft}{ }^{-3}\right]$, is a function of the droplet mass and vapor/droplet volume:

$$
C=\frac{\alpha_{e} \rho_{l}}{\alpha_{e}+\alpha_{v}}
$$

The mass transfer coefficient, $k_{0}$, was correlated by Whalley[52] as a function of surface tension given by

$$
k_{0}=\max \left\{\begin{array}{c}
3.0492 \times 10^{12} \sigma^{5.3054} \\
12.491 \sigma^{0.8968}
\end{array}\right.
$$

with $k_{0}$ in $\mathrm{ft} \mathrm{s}^{-1}$ and $\sigma$ in $\mathrm{lbf} \mathrm{ft}^{-2}$.

Other terms in the correlation include wetted perimeter, $P_{w}$, and cell height, $\Delta X$.

\subsubsection{Bottom Reflood Entrainment}

Entrainment The bottom reflood process involves the quenching of fuel rods that existed in a hot wall flow regime. Film boiling occurs and, as the rods cool, transition boiling and, finally, nucleate boiling take place. These processes cause a large vapor generation rate and a corresponding high vapor velocity which causes a large amount of droplet entrainment. The actual entrainment mechanisms can be a result of liquid 
core breakup due to surface instabilities in the case of subcooled liquid or due to bubbles breaking through the liquid core surface in the case of saturated liquid. The model used in CTF is based on the work of Kataoka, Ishii, and Mishima[53], which considers droplet entrainment caused by vapor bubbling through liquid pools.

$$
S_{E}=\left(\alpha_{v} \frac{U_{v}}{U_{\text {crit }}}\right)^{2} \dot{m}_{v}
$$

The actual form used in CTF is:

$$
S_{E}=1.5 \min \left[2.5,\left(\frac{U_{v}}{U_{\text {crit }}}\right)^{2}\right] \dot{m}_{v}
$$

The critical velocity, $U_{\text {crit }}$, is the vapor velocity required to lift a droplet with radius defined by the critical Weber criterion against the pull of gravity. It is defined as follows:

$$
U_{\text {crit }}=\left(\frac{4 W e_{d}}{3 C_{D, d}}\right)^{1 / 4}\left(\frac{\sigma g^{2} \Delta \rho}{\rho_{v}^{2}}\right)^{1 / 4}
$$

The Weber number, $W e_{d}$, is taken to be 2.0 as a result of reflood experiments in the FLECHT tests. The droplet drag is taken to be 0.45 . The density difference, $\Delta \rho$, is the difference between the liquid and vapor density. The droplet flow rate entering the cell is subtracted from the entrainment rate. It is further multiplied by a factor to account for low liquid void fractions.

$$
S_{E}=\max \left[0, S_{E}-\dot{m}_{e}\right] \min \left[1,5 \alpha_{l}\right]
$$

De-Entrainment De-entrainment is not considered for the case of bottom reflood.

\subsection{Top Down Reflood Entrainment/De-Entrainment}

Entrainment Top down reflood occurs due to upper plenum injection during accident conditions. After the core has dried out and hot wall conditions exist, the core may be quenched from coolant injection that falls from above the core. Two mechanisms for entrainment are compared for this case. The first mechanism involves film falling faster than the downward moving quench front. If liquid moves lower than the quench front into hot wall regions, the liquid will quickly sputter, breaking up into droplets. The second mechanism of entrainment is caused by entrainment off of the film surface due to upward flowing vapor. The countercurrent flow entrainment model that was used for the annular/mist regime (Section 5.3.2) is used for the second mechanism.

For the first mechanism, we can assume that all film that falls to the quench front and isn't vaporized, is entrained. This can be written as follows: 


$$
S_{E}=-\dot{m}_{l}-\Gamma_{q}
$$

The mass flow rate of the liquid film that reaches the quench front is $\dot{m}_{l}$ and the vapor generation rate at the quench front is $\Gamma_{q}$. The vapor generation rate is simply the old time step vapor generation rate for the cell in which the quench front resides. The mass flow rate is the old time step liquid mass flow rate into the cell in which the quench front resides. The liquid mass flow rate will be negative in CTF since the film is falling, hence the preceding negative in the above equation.

The second mechanism uses Equation 5.82 from Section 5.3.2, repeated below:

$$
S_{E, \text { coutner }}=\left(\alpha_{l}-\alpha_{l, \text { crit }}\right) \rho_{l}\left|U_{l}\right| A_{x}
$$

Substituting the definition of the critical void fraction leads to the following equation:

$$
S_{E}=\left(\alpha_{l}-\frac{2 \sigma}{D_{h} \rho_{v} \vec{U}_{v l}^{2}}\right)\left|\dot{m}_{l}\right|
$$

The maximum of the two entrainment model results is taken as the entrainment rate for the falling film. Some additional considerations are then made. If the void fraction is less than 0.8 , the deludge flow regime is considered. If void fraction is less than 0.6 , the entrainment rate is zeroed. If void fraction is between 0.6 and 0.8 , a ramp is used to alter the falling film entrainment rate - the rate is ramped between $0 \%$ for void fractions of 0.6 and $100 \%$ for void fractions of 0.8 . The ramp function is:

$$
S_{\text {ramp }}=\max \left[0.0, \min \left(5\left(\alpha_{v}-0.6\right), 1.0\right)\right]
$$

For void fractions greater than 0.8 , an additional consideration is made where the maximum of the falling film entrainment rate and the liquid mass flow rate is taken for the entrainment rate:

$$
S_{E}=\max \left[0.0, S_{E},-\dot{m}_{l}\right]
$$

De-Entrainment The de-entrainment in the top down reflood region is assumed to be caused by random turbulent motions of the drops, which causes drops to impact the film and be absorbed. The same model that was used for the annular/film region is used here. The model of Cousins[51] is repeated:

$$
S_{D E}=k_{0} \Delta C P_{w} \Delta X
$$

Definition of the equation terms can be found in Section 5.3.2. One modification is made to Equation 5.91 to account for the quench front. Only droplets impacting the film are de-entrained, so it is necessary to know the fraction of the cell which is quenched to determine the de-entrainment rate. This fraction is multiplied by the Cousins model. 


$$
S_{D E}=k_{0} \Delta C P_{w} \Delta X F_{q}
$$

The fraction of the cell that is quenched, $F_{q}$, is calculated:

$$
F_{q}=\frac{X_{b}-X_{q f}}{\Delta X}
$$

The top of the momentum cell - the global location of the boundary between adjacent continuity mesh cells - is $X_{b}$ and the code-calculated quench front global location is $X_{q f}$. The difference of these values is the length of the quenched portion, which is divided by the momentum cell height to get the quenched fraction of the heater rods.

\subsubsection{Crossflow De-Entrainment}

The crossflow de-entrainment is modeled by considering flow across tube banks. De-entrainment is calculated for each gap connecting to a channel using a rod-bundle de-entrainment efficiency term, $\eta_{D E}$.

$$
S_{D E}=\frac{1}{2} \eta_{D E}\left|\dot{m}_{e, Z}\right|
$$

The rod bundle de-entrainment efficiency term is user-input. The lateral droplet mass flow rate through the gap, $\dot{m}_{e, Z}$ is used. The de-entrainment rate is calculated for each gap connecting to a channel and a total de-entrainment rate is calculated by summing the individual gap contributions together.

\subsubsection{De-Entrainment in Area Changes}

Droplets will de-entrain if they move through a change in flow area, forming a film on the surface. This effect is most prominent during accident conditions when entrained droplets flow through the upper core tie plate. Droplets will de-entrain when moving through the plate and form a film which will provide the initial liquid for the top quench front. De-entrainment is captured by simply using the ratio of the areas.

$$
S_{D E}=\max \left[0.0, \dot{m}_{e, j-1}\right]\left(1-\frac{A_{j}}{A_{J}}\right)+\max \left[0.0,-\dot{m}_{e, j}\right]\left(1-\frac{A_{j-1}}{A_{J}}\right)
$$

The equation has two components to account for the possibility that entrained flow is moving in the top down direction - one of the components will be zero. The ratio between momentum cell and continuity mesh cell area is taken. Due to the staggered mesh approach, the momentum cell will lie on the top boundary of the continuity mesh cell with the same level index. 


\subsubsection{De-Entrainment at Solid Surfaces}

It is assumed that droplets that move normal to a solid surface are de-entrained on the surface. Furthermore, droplets that flow into a bubbly flow regime are also assumed to be de-entrained. This is handled in $\mathrm{CTF}$ by checking if a mass flow boundary condition was chosen for the momentum cell above or below the continuity

mesh cell. A mass flow boundary condition would imply that there is a solid surface from which the injection is occurring. If a mass flow boundary condition is in the momentum cell above, the entrainment rate is:

$$
S_{D E}=\max \left[0.0, S_{D E, \text { cousins }}, \dot{m}_{e, j-1}\right]
$$

The model of Cousins[51] is used for comparison against the entrained droplet flow rate. The model is in the same form as was used for the top down reflood de-entrainment rate calculation. For a mass flow rate boundary condition below the continuity mesh cell, the negative of the droplet mass flow rate in the momentum cell above is used.

$$
S_{D E}=\max \left[0.0, S_{D E, \text { cousins }},-\dot{m}_{e, j}\right]
$$

Consideration is made for de-entrainment in the wet film on quenched unheated conductors, should they exist. One third of the droplets are de-entrained on a quenched housing, as follows:

$$
S_{D E, h}=\frac{1}{3} X_{q} P_{w, c} \Delta X \frac{\alpha_{e} \rho_{l}}{\alpha_{e}+\alpha_{l}}
$$

The fraction of the conductor which is quenched is $X_{q}$ and the wetted perimeter of the housing is $P_{w, c}$. The quenched housing de-entrainment rate is added to the solid surface de-entrainment rate.

$$
S_{D E}=S_{D E}+S_{D E, h}
$$

\subsubsection{Spacer Grid Droplet Breakup Model}

Dispersed droplets in the flow field can be shattered as they impact spacer grids. Experiments performed by the Central Electricity Generating Board of the United Kingdom[54], the University of New York at Stony Brook[55], and Westinghouse/Carnegie-Mellon University[56] show that this breakup phenomenon can be significant. It is important to capture this phenomenon because the resulting field of smaller droplets evaporates more easily than larger droplets, which reduces vapor superheat and improves rod heat transfer during accident conditions. To capture this phenomenon, droplet breakup due to the spacer grid is determined and the increase in small droplet interfacial area is calculated, which is added to the interfacial area source term in the interfacial area transport equation (see Section 3.4).

The spacer grid droplet breakup model can be found in the GRID subroutine. Calculations are only performed if less than $99 \%$ of the grid height is quenched. The goal of the grid droplet breakup model is to provide the interfacial area transport equation with information on the rate of small droplet creation and 
the small droplet diameter such that the effect on droplet interfacial area can be determined. CTF actually considers two stages of breakup: the breakup of large droplets into small droplets and the breakup of small droplets into micro droplets. In the end, however, the small droplet and micro droplet number fluxes (breakup rate) and diameters are merged into one set of information for a single small droplet field.

The large droplet field breakup is considered first. The number of droplets in the flow field that break on the grid strap are calculated as a function of the grid blockage ratio and the droplet flow rate.

$$
\dot{m}_{d b}=\eta\left(\frac{A_{x, g}}{A_{x}}+\frac{1}{2} \frac{A_{x, s}}{A_{x}}\right) \dot{m}_{e}
$$

The leading factor, $\eta$, is a breakup efficiency factor - it is estimated to be 0.6. The area blockage ratio is the sum of the grid strap blockage ratio and half of the grid spring flow blockage ratio. With the droplet breakup rate, it is necessary to then calculate the new droplet diameter so that the interfacial area source may be calculated. Droplet breakup data taken from Wachters ([57] and [58]) and Takeuchi[59] was used to characterize the Sauter mean diameter of shattered droplets as a function of initial droplet diameter and original droplet Weber number. The correlation of the data can be seen in Figure 5.1.

The correlation for the diameter of the shattered droplets is:

$$
\frac{D_{s d}}{D_{i}}=6.167 W e_{d}^{0.53}
$$

At low droplet Weber numbers, Equation 5.111 will return shattered droplet diameters that are close to the incident droplet diameter. Such droplets, having large diameters, should not be considered in the small droplet field. Therefore, for $W e_{d}<30.9$, the calculated large droplet breakup is not considered to enter the small droplet field. Instead, the code checks for small droplet breakup into micro droplets. For $W e_{d}>250$, all large droplets that shatter (calculated by Equation 5.110) are considered to enter the small droplet field and the small droplet field number flux is calculated accordingly. For $30.9<W e_{d}<250$, a linear interpolation is performed between zero large droplets breaking up into small droplets and the full value calculated by Equation 5.110. The ramping factor that is multiplied by the droplet breakup rate is:

$$
R=\frac{W e_{d}-30.9}{250-30.9}
$$

A similar check is done for the small droplet field breakup. If the small droplet Weber number is less than 30.9, no small droplets are considered to breakup into micro-droplets. If, however, $W e_{d}>30.9$, then the small droplets break on the spacer at the full droplet breakup rate. The logic for this approach is best summarized in a flowchart, shown in Figure 5.2.

In Figure 5.2, nsd is the droplet number flux — it is the number of droplets entering a droplet field per unit time due to shattering from larger droplets. The shattered droplet diameter is represented with the $d s d$ term. The droplet number flux and shattered droplet diameter are initialized to 0 and $1 \cdot 10^{-8}$, respectively.

The large, incident, droplet Weber number is calculated first. If it is smaller than 30.9, then the small droplet number flux stays at zero since any large droplets that do shatter are shattered into large droplets which remain in the large droplet field. Otherwise, the small droplet mass flow rate is calculated by Equation 


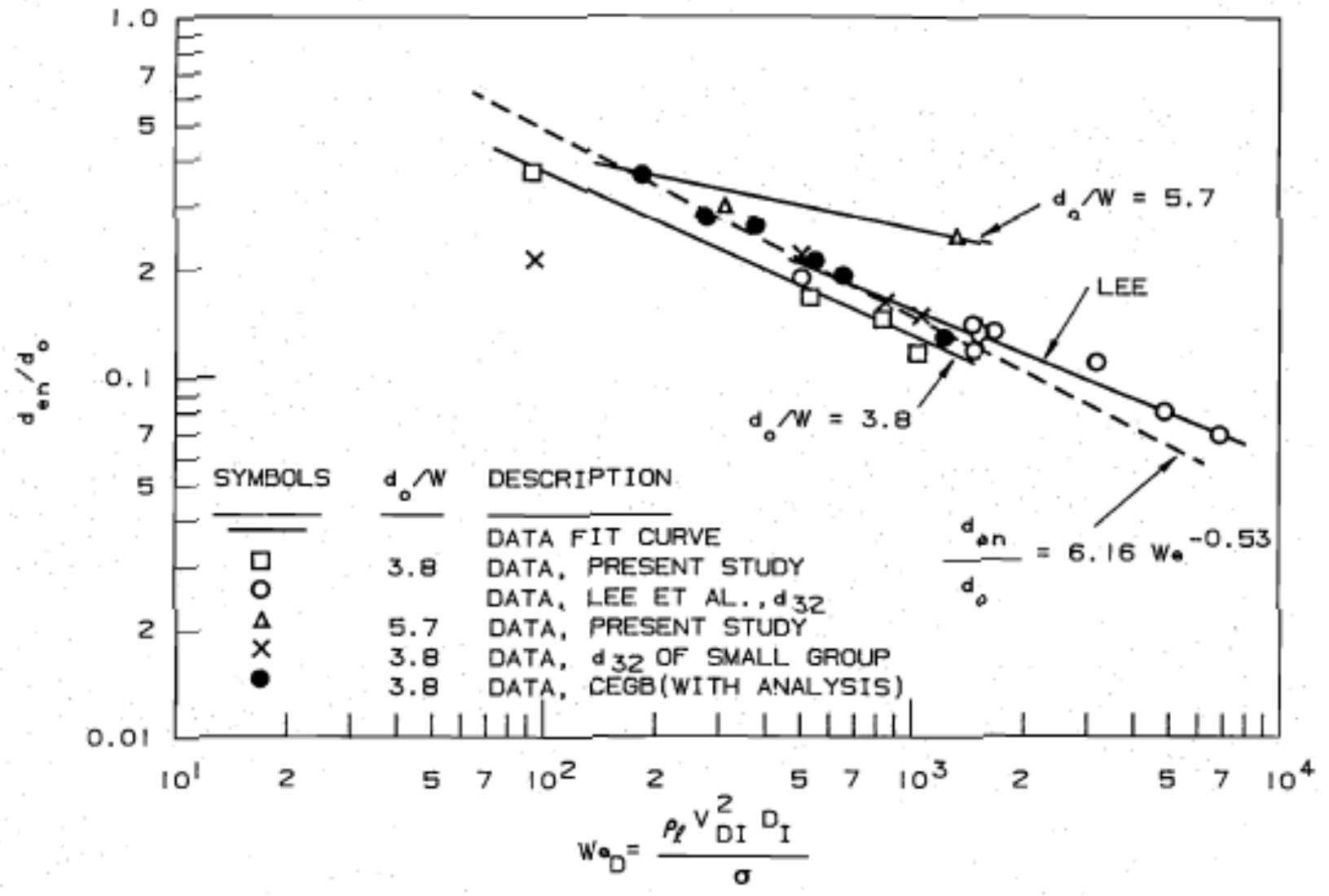

Figure 5.1: Ratio of shattered droplet diameter to incident droplet diameter dependence on droplet Weber number 


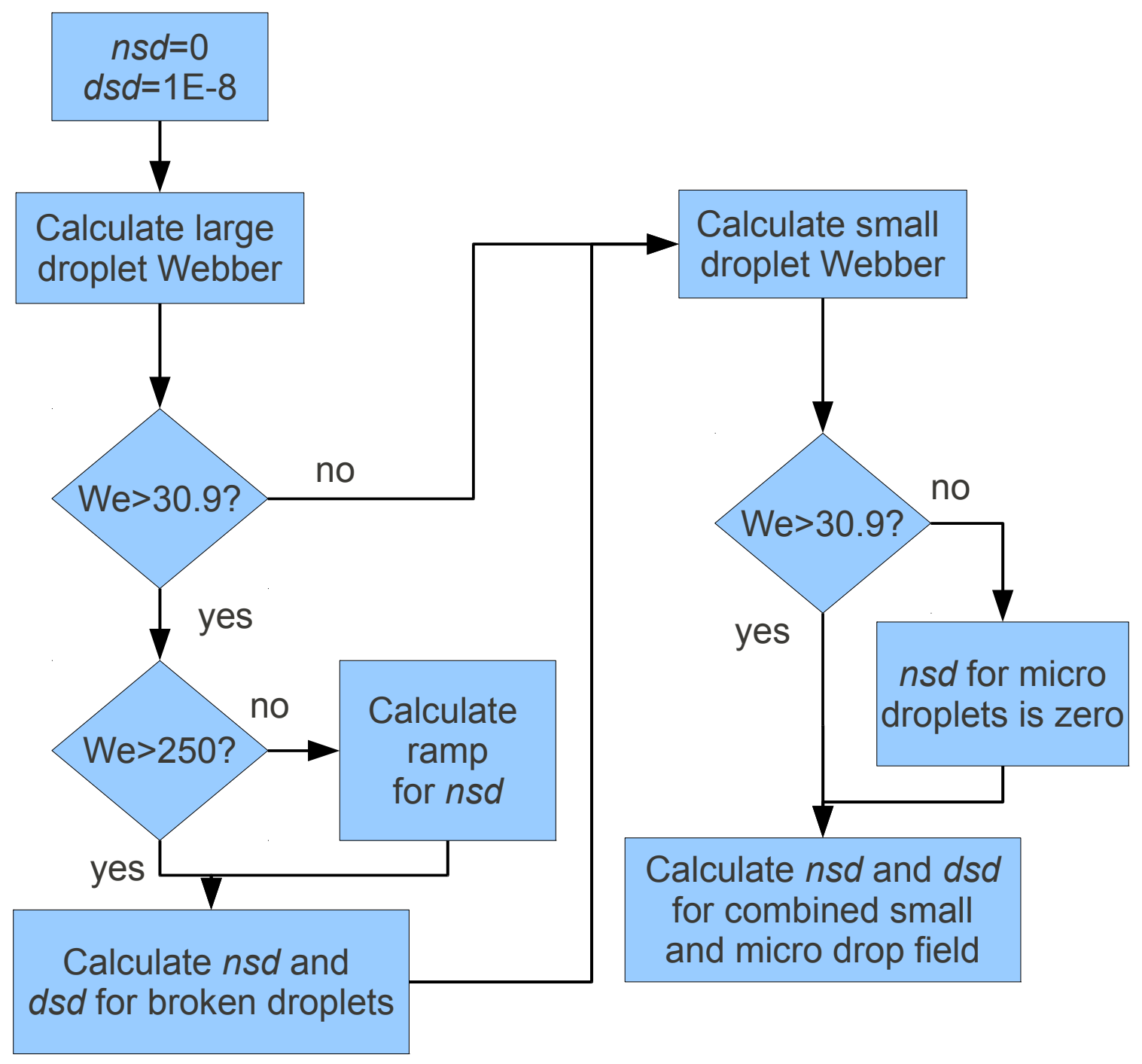

Figure 5.2: Flowchart for the spacer grid droplet breakup model found in the GRID subroutine 
5.110. The droplet number flux is then calculated using the mass flow rate from the large to small droplet field and the mass of a single small droplet.

$$
n s d=\frac{\dot{m}_{d}}{m_{d, \text { single }}}
$$

The single drop mass is calculated from the shattered droplet Sauter mean diameter of a droplet, obtained from Equation 5.111, which is used to calculate the droplet volume. The droplet volume is multiplied by the liquid density to obtain the droplet mass.

$$
n s d=\frac{\dot{m}_{d}}{1 / 6 \pi d_{s d}^{3} \rho_{l}}
$$

For $W e_{d}<250$, a ramping factor is calculated by Equation 5.112 and multiplied by the small droplet number flux to reduce the number of shattered droplets that enter the small droplet field.

This process is repeated for the small droplet field. It is important to note here that the shattered droplet number flux is defined on a per channel basis. Therefore, the shattered droplet number flux includes droplets that were shattered not only by the current grid being analyzed, but also the upstream grid (if one exists) that caused droplet shattering. Even if the large droplet Weber number was smaller than 30.9 and no large droplets shattered on the current grid entered the small droplet field, there may still be small droplets that shatter into micro droplets due to shattering from upstream grids.

After the small to micro droplet shattering rate is determined, the small and micro droplet number flux and diameters are combined into a single set of values. This is done for the shattered droplet number flux by first adding the small and micro droplet field mass flow rates. Second, Equation 5.114 is used to calculate the number flux for the combined small and micro droplet field, but using the Sauter mean diameter of the combined fields.

The Sauter mean diameter of the combined fields is calculated by a mass flow rate averaging process. The small droplet mass flow rate is divided by the small droplet diameter and, likewise, the micro droplet mass flow rate is divided by the micro droplet diameter. These terms are added and divided into the total small droplet and micro droplet mass flow rate to get the Sauter mean diameter of the combined fields. This is shown below.

$$
d_{s d, \text { combined }}=\frac{\dot{m}_{\text {small }}+\dot{m}_{\text {micro }}}{\frac{\dot{m}_{\text {small }}}{d_{\text {small }}}+\frac{\dot{m}_{\text {micro }}}{d_{\text {micro }}}}
$$




\section{Heated and Unheated Conductor Mod- els}

\subsection{Introduction}

Thus far, emphasis has been placed on modeling of the flow field using conservation equations and associated models for conservation equation closure terms. CTF also contains a capability for modeling solid components such as fuel rods, electric heaters, and unheated conductors. This chapter discusses these models.

A flowchart for the general solution of conductor temperature profiles is shown in Figure 6.1- the flow of source code subroutines is presented. Subroutine HEAT is called at the end of PREP3D, which means that the heated/unheated conductor temperature profiles are solved prior to the new time flow field. Subroutine QFRONT locates the quench front (if one exists) and performs re-noding of the rod to make for a more accurate quench front solution. The code loops over all channels and all axial levels and calls BOILING and HCOOL. The BOILING subroutine calculates the CHF point and HCOOL calculates the heat transfer coefficient for the conductor. Subroutine RADIANT calculates radiation heat transfer, if selected by the user and TEMP calculates the temperature profile throughout the conductor.

The models used for tracking the quench front were discussed in Section 6.6.11.3, but the re-meshing of conductors was not discussed - re-meshing is discussed in this chapter. The location of the CHF point and the calculation of the CHF temperature will be discussed in Section 6.5 and, specifically, Sub-section 6.5. Wall convective heat transfer models will be discussed in Section 6.6. Radiative heat transfer models have been discussed in Section 6.6.10. This leaves determination of the heat transfer through the conductor and the resulting temperature profile (subroutine TEMP), which is discussed further in this chapter.

There are two general types of conductor models included in CTF: one for rods and one for generic conductors. The rod models can be used for modeling solid or hollow heater rods and also nuclear fuel rods (models are included for gap conductance, fuel rod deformation, and heat generation accounting for nuclear reactions as well as metal-water reaction in cladding). Rods can be active or passive (i.e. heated or unheated) and they may exceed the CHF temperature and, thus, require the CHF models. Generic conductors, however, are always considered passive and have no internal heat sources. Furthermore, they need not be of cylindrical geometry and it is assumed that they always remain below the CHF temperature and therefore never require the use of the CHF models.

The common feature of the different conductor type models is the conduction equation - it is discussed first 


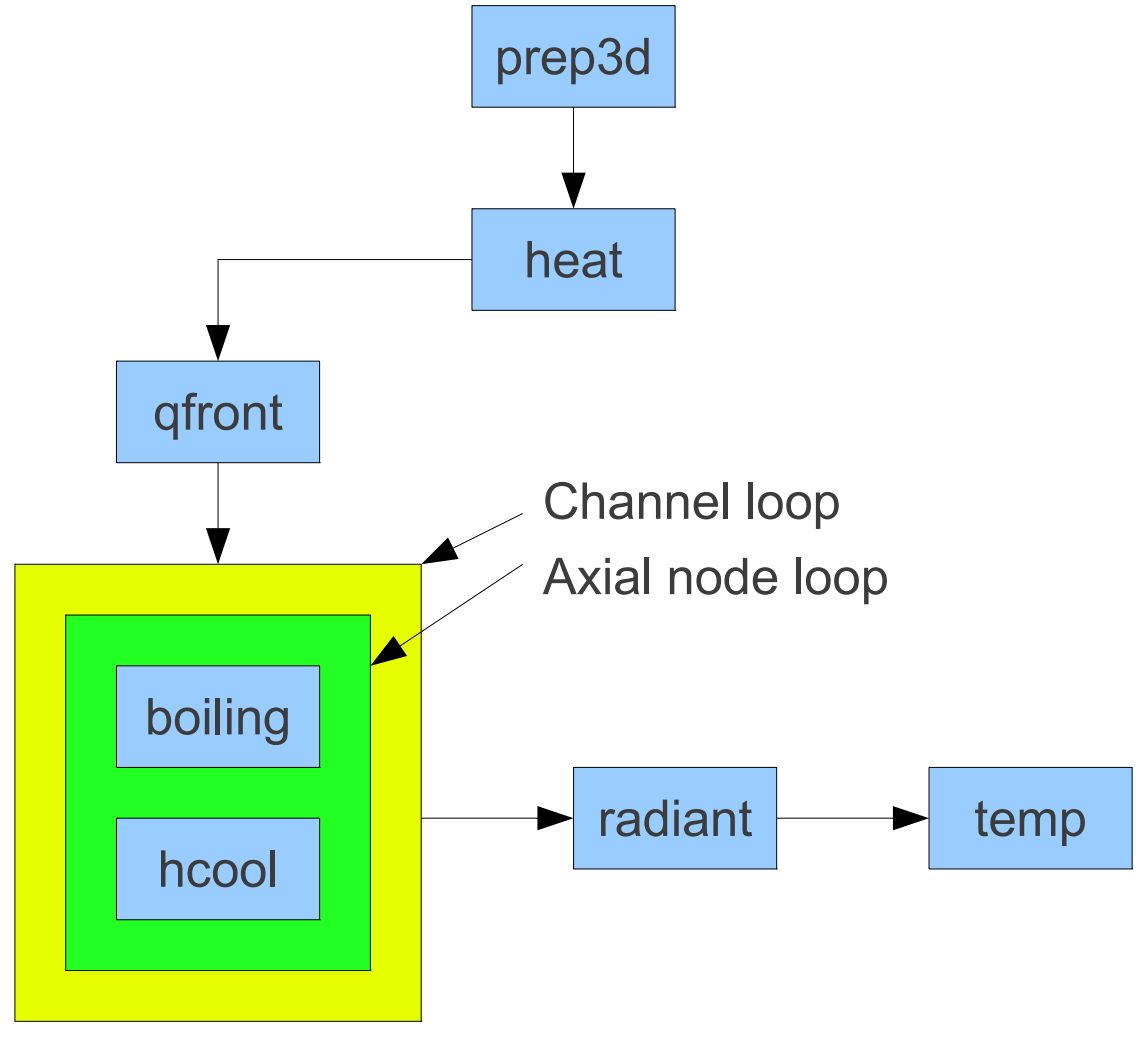

Figure 6.1: Flowchart for heated and unheated conductor temperature solution 
in the following section. After this, the conductor re-meshing package is discussed, which is used to refine or collapse the mesh used for the conductors depending on the location of the quench front. These are the only models that are used for modeling generic conductors or solid or hollow heater rods. Nuclear fuel rods, however, require special considerations which are discussed in the final section, Section 6.4.

\subsection{Conduction Equation}

\subsubsection{Formulation of the General Conduction Equation}

CTF includes capabilities for modeling fuel rods, electric heater tubes, and walls using a finite-difference form of the heat conduction equation which is formulated using the heat balance approach[60]. This approach breaks the conductor into control volumes with nodes being located at the control volume centers or surfaces.

An example of this meshing approach is shown for a cylindrical geometry in Figures 6.2 and 6.3. Figure 6.2 shows both side and top views of a meshed nuclear fuel rod. The rod is divided into 4 axial levels, with $j$ being the index for axial level. Each axial level of the rod will be meshed into both radial and azimuthal segments. Figure 6.3 provides a closer look at the internal meshing of the fuel rod for a single axial level.

The rod is divided into 4 azimuthal segments, labeled using $k$. The orange and green boxes highlight two of the azimuthal segments, $k=1$ and $k=2$. Note that an azimuthal segment will be created for each $\mathrm{rod} /$ channel connection. If a pin-centered subchannel approach were employed, where each rod connects to only one channel, there would be only one azimuthal segment in the rod. Also note that azimuthal segments will be indexed in CTF according to the indexing of channels and rods, so the labeling presented in Figure 6.3 is for example only.

Figure 6.3 additionally gives an example of the radial meshing of the fuel rod. The fuel pellet is represented in red, the gap is white, the clad is blue, and the optional Crud layer is grey. The fuel pellet is divided into three mesh cells. The clad is divided into two regions and the Crud layer is represented using only one region. For a nuclear fuel rod, the radial meshing (number of mesh cells) of the fuel pellet can be controlled by the user via input; however, the number of regions for other areas (gap, clad, and Crud) are controlled by CTF.

Attention is now given to the node locations. Nodes inside the fuel pellet will always be in the center of the mesh cell volume for internal mesh cells. The fuel pellet surface mesh cell, however, will have its node on the surface of the fuel pellet. The two clad mesh cells will have their nodes on the opposite surfaces of the clad. Likewise, the Crud layer will have its node on the fuel rod surface. Shifting the node locations from mesh-cell center to surface allows for CTF to define a temperature at needed locations (i.e. fuel pellet surface, clad inside and outside surfaces, and fuel rod surface).

A non-nuclear cylindrical geometry will be discretized similarly. The primary differences will be that: (1) the user may divide the rod into any number of regions and (2) each region may have any number of nodes. Unheated conductors, or "slabs", have a plate-like geometry. The $i$ index counts nodes across the wall thickness, $j$ in the axial direction, and $k$ indices count along the wall width in the lateral direction. Meshing the geometry using this control volume heat balance approach allows for:

- unequal mesh spacing 


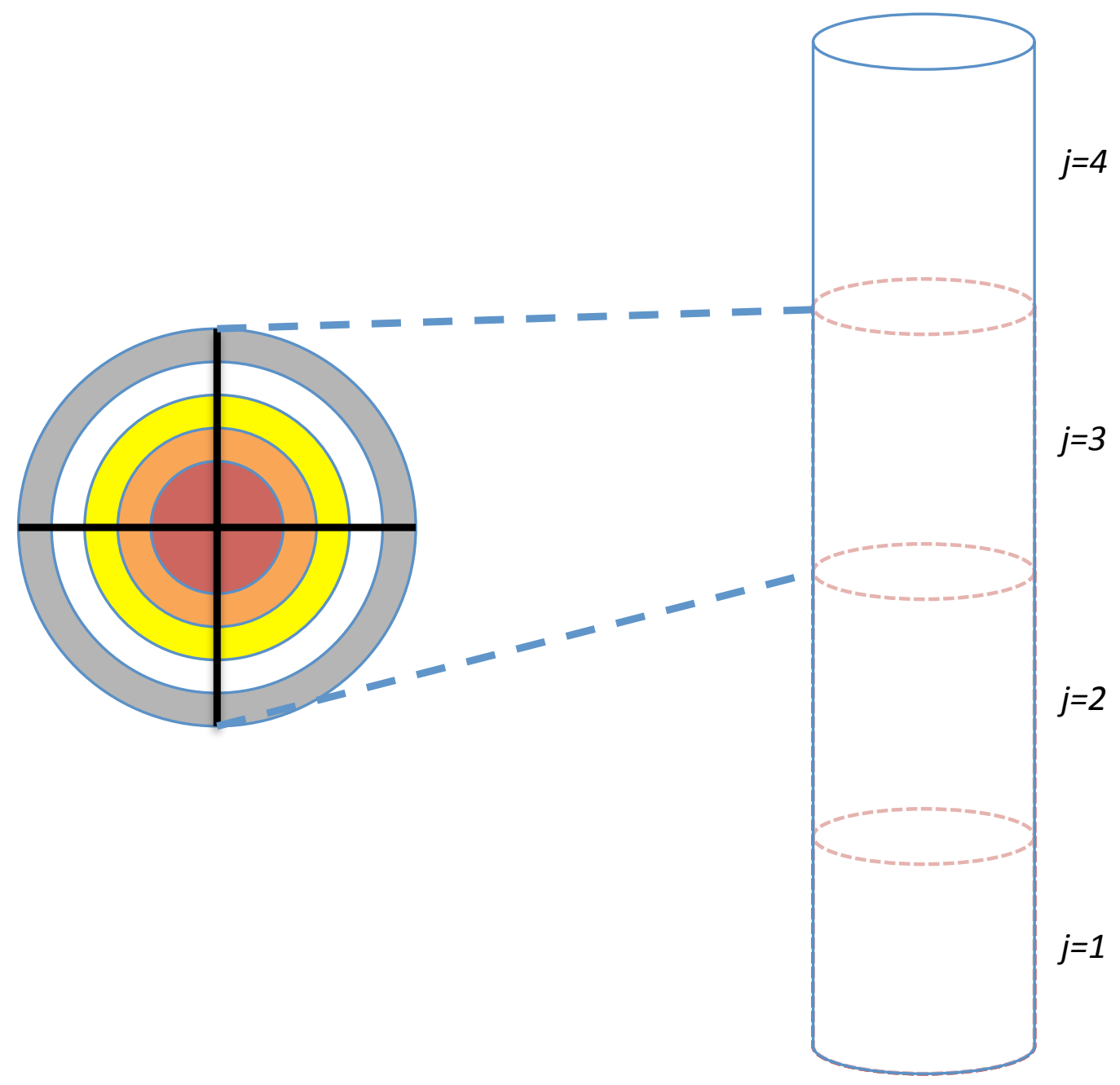

Figure 6.2: General CTF meshing approach for nuclear fuel rod 


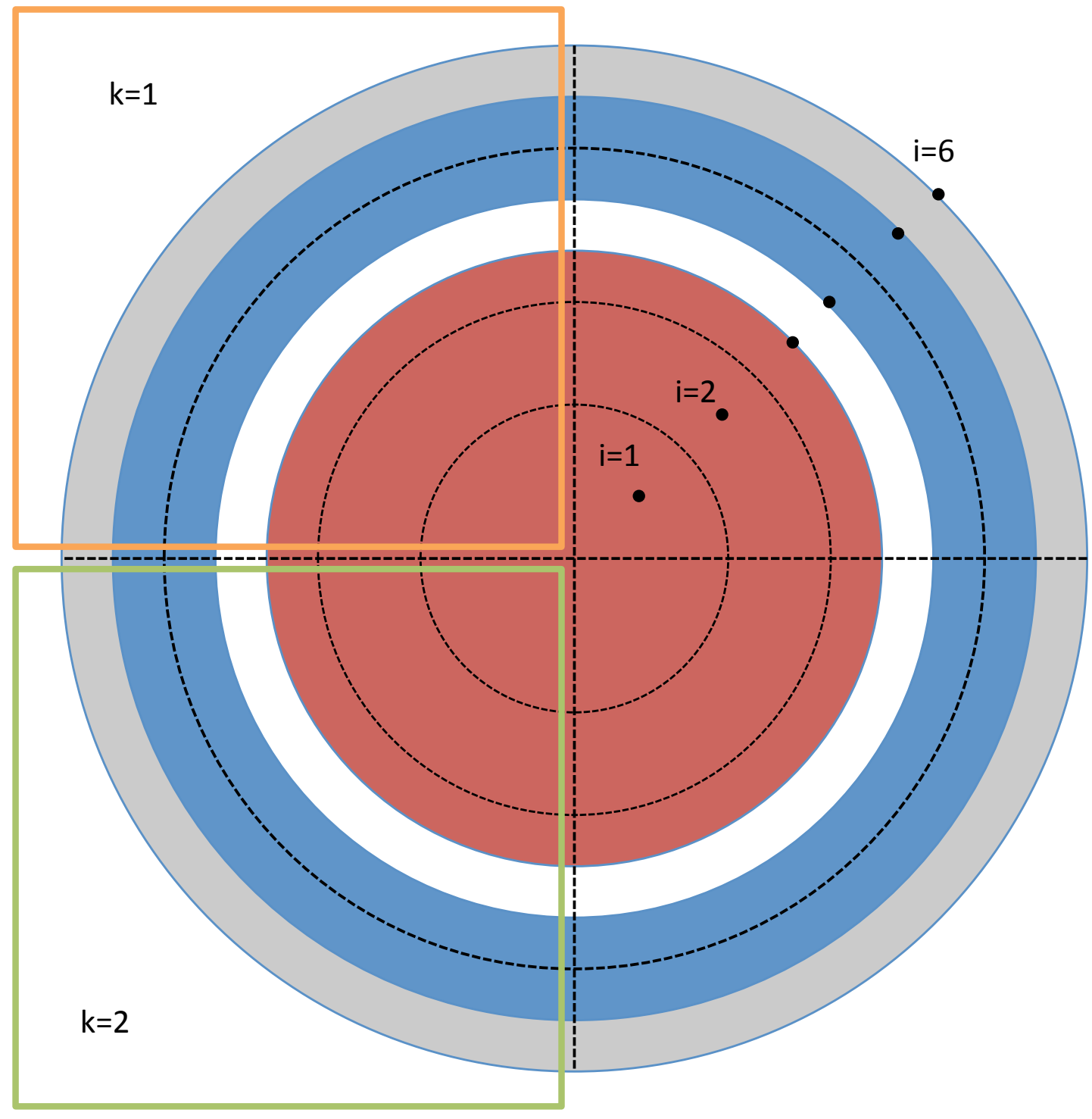

Figure 6.3: A closer look at CTF internal meshing of nuclear fuel rod 
- temperature dependent material properties

- space dependent material properties

- changes in internal resistance to heat transfer (e.g. gaps)

- changes in internal heat generation.

The general conduction equation for a solid mesh cell is shown in Equation 6.1.

$$
\frac{d}{d t} \int_{V} \rho C_{p} V T=\oint_{A} \boldsymbol{n}_{k} Q_{k} d A+\int_{V} Q^{\prime \prime \prime} d V-\oint_{A} Q_{s} d A
$$

The LHS term is the transient term, with $\rho$ being material density, $C_{p}$ being material specific heat, $T$ being the material temperature, and $t$ being the timestep size of the solid conduction equation solution. On the RHS, the first term is a surface integral over the solid mesh cell that accounts for energy conduction in axial, azimuthal, and radial directions. The $\boldsymbol{n}$ is the unit vector orthogonal to the surface, $k$ is the current surface, and $A$ is the surface area of the solid mesh cell. The second term represents volumetric generation of energy due to fission or electrical heating with $Q^{\prime \prime \prime}$ being the volumetric energy generation rate and $V$ being the cell volume.

The third, and final, term of the equation is the convective heat transfer at the cell surface which transports energy from the solid to surrounding fluid or vice versa. Note that only mesh cells exposed to the coolant will have this final term. Furthermore, only mesh cells having some heating source will have the volumetric energy generation term. For a nuclear fuel rod, energy generation can include the heat generation due to fission in the pellet as well as heat generation in the clad due to zirconium-water reaction. Energy generation in solid and hollow electric heater rods comes from electrical heating. Rod heat rates may be dependent on radial and axial location in the rods and are input by user. For unheated conductors, the energy generation term remains absent.

Note that the mass of the control volume (i.e. $\rho V$ ) is always evaluated at the cold-state properties. This is due to the fact that the nodal locations are fixed once they are defined in CTF while, in reality, they may move due to thermal expansion. If $\rho V$ were allowed to change with temperature without allowing the control volume dimensions to also change, a mass imbalance would occur.

The CTF rod solution algorithm works by looping over each rod, then each axial level of the rod, then each azimuthal segment at that level. For a single segment, CTF then loops over each radial node and sets up

the terms that will form the equation for that node. At the end of each radial node loop, the system of equations will be solved by Gaussian elimination. Therefore, the rod conduction solution is implicit in only the radial direction; azimuthal and axial conduction terms are added to the conduction equations explicitly.

In the CTF solution, the thermal conduction term, $Q_{k}$, will be expanded for each surface of the current cell being setup. Each conduction term will take on the form shown in Equation 6.2, where $a$ represents the mesh cell being solved and $b$ represents a connected mesh cell.

$$
Q_{a \rightarrow b}=k_{a b}\left(T_{b}-T_{a}\right)
$$


Both $T_{a}$ and $T_{b}$ will be unknowns if Cells $a$ and $b$ are connected in the radial direction. If either cell is connected in the axial or azimuthal direction, the temperature of that cell will be explicitly given from a previous time step. The average thermal conductivity between Cell $a$ and $b$ will be some combination of the individual thermal conductivities of the two cells, $k_{a}$ and $k_{b}$. This averaged thermal conductivity is defined using the concept of thermal resistances as shown in Equation 6.3, where $R_{a \rightarrow \text { boundary }}$ is the thermal resistance from the node of Cell $a$ to the boundary between Cells $a$ and $b$ and $R_{\text {boundary } \rightarrow b}$ is the thermal resistance from the boundary to the node of Cell $b$.

$$
k_{a b}=\frac{1}{R_{a \rightarrow \text { boundary }}+R_{\text {boundary } \rightarrow b}}
$$

The thermal resistances can be defined for cells connected by a varying surface area (e.g. cylindrical mesh cells connected in the radial direction) or a constant surface area (e.g. rectangular mesh cells or cylindrical mesh cells connected in the axial or azimuthal directions). An example of a system of thermal resistances for a cylindrical geometry is shown in Figure 6.4.

In this example, the fuel rod is broken into 6 mesh cell volumes, each with a node, labeled as N1-N6, that is either at the mesh cell center or surface. There are, as a result, 8 thermal resistances, labeled as R1-R8. Note that cells having a node at their center will have two thermal resistances; one to communicate with each cell boundary. Since the Crud layer has only one node at its outside surface, it requires only one thermal resistance.

The formulation for the above thermal resistances (radial direction) will be that of a cylindrical geometry as shown in Equation 6.4.

$$
R=\frac{\ln \left(\frac{r_{o}}{r_{i}}\right)}{2 \pi k L}
$$

Here, $r_{o}$ and $r_{i}$ are the outside and inside radii of the cylindrical mesh cell, $k$ is the thermal conductivity, and $L$ is the axial height of the cell. Figure 6.5 shows an example of the resistance in the axial direction for a nuclear fuel rod. Since heat transfer in the axial and azimuthal directions is through a constant area, $A$, the resistance is linear and is defined as shown in Equation 6.5.

$$
R=\frac{L}{k A}
$$

The fact that azimuthal and axial conduction and the convective boundary term are all explicit in the solid governing equation means that some controls are needed to keep the solution stable. Additionally, the CTF solid modeling capabilities include some features for capturing behavior of nuclear fuel rods, such as changes in gap conductance due to rod geometry changes, and changes in fuel pellet conductivity due to cracking and sintering. These matters are discussed in the following sections along with the concept of thermal resistances. 


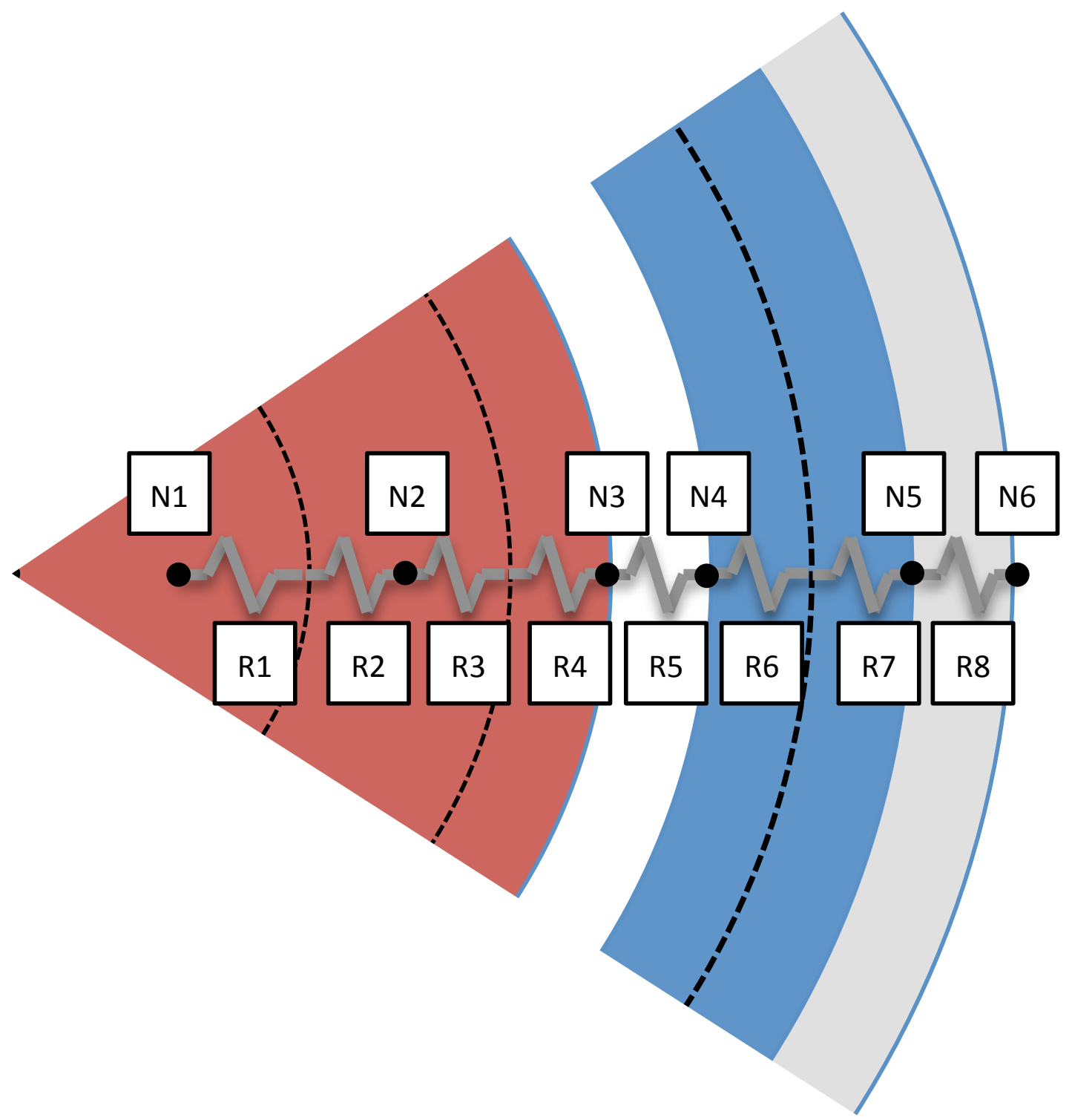

Figure 6.4: CTF model for fuel rods as a thermal resistance network 


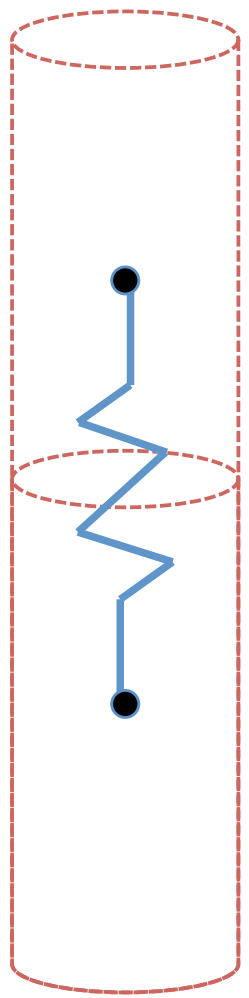

Figure 6.5: Example of resistance in the axial direction for nuclear fuel rod 


\subsubsection{Conductor and Flow Field Boundary}

The conductors modeled by CTF must contact the fluid field at some, if not all, of their surfaces. Any conductor surface that doesn't join a fluid mesh cell either contacts another conductor, transfers heat to some outside environment, or is assigned an adiabatic boundary condition and transfers no heat. The conductors are coupled to the flow field solution by three possible conditions in CTF: convective heat transfer to the fluid, heat transfer due to droplet impingement and vaporization, and radiative heat transfer to the fluid and other bodies around the conductor. A heat flux is calculated for each of these effects and it is added to the conduction equation for surface cells that contact the fluid. If the conductor surface does not exchange heat with the fluid field, CTF has an option for the user to prescribe a heat transfer coefficient and sink temperature for modeling heat loss to some external environment.

The calculation of heat transfer coefficients based on heat transfer regime will be discussed in Section 6.6. The radiative heat transfer modeling has been discussed in Section 6.6.10. It is important here to also discuss the general approach for applying these terms in calculating heat flux and the special consideration that must be made for the nucleate boiling regime. This is discussed in Section 6.2.2.1. The third vector for heat transfer between rod and fluid, droplet impingement heat transfer at flow blockages, is discussed in Section 6.2.2.2.

\subsubsection{Convective Heat Transfer}

Since the fluid may be two-phase, the heat flux is a sum of heat transfer to liquid and vapor phases.

$$
q^{\prime \prime}=H_{l}\left(T_{s}-T_{l}^{n}\right)+H_{v}\left(T_{s}-T_{v}^{n}\right)
$$

Heat transfer coefficients for the liquid and vapor phases are represented by $H_{l}$ and $H_{v}$. The conduction equation solution is performed prior to the flow-field solution for any given timestep. Therefore, the conductor cell temperature, $T_{s}$, is the current time-step surface temperature of the conductor while the liquid and vapor fluid temperatures are obtained from the old timestep (hence the $n$ superscript). Section 6.6 discusses the wall heat transfer models and calculation of the $H_{l}$ and $H_{v}$ heat transfer coefficients for all heat transfer regimes modeled by CTF.

Before Equation 6.6 can be added to the conduction equation, special consideration must be made for the heat flux to the liquid phase. For the case of nucleate boiling, there is a strong dependency between wall surface temperature and heat flux. Therefore, the wall heat transfer and surface temperature are coupled implicitly in a non-iterative manner by considering the linearized derivative of the heat transfer coefficient with respect to wall temperature when calculating the heat flux to be used as the wall boundary condition. The liquid heat flux becomes:

$$
q_{l}^{\prime \prime}=H_{l}\left(T_{s}-T_{l}^{n}\right)+\left(\frac{\partial H_{l}}{\partial T_{s}}\right)\left(T_{s}-T_{s}^{n}\right)\left(T_{s}^{n}-T_{l}^{n}\right)
$$

In this manner, the change in the heat transfer coefficient from the previous timestep is considered in calculating the new surface temperature. This modified heat flux is added to the conduction equation. 


\subsubsection{Droplet Impingement Heat Transfer}

The droplet impingement heat transfer model is for a special scenario where accident conditions cause the localized swelling of fuel cladding. As cladding swells into the flow channel, the cladding of different rods can meet and cause a blockage of the flow. Droplets that are entrained in the vapor flow can impact the swollen cladding. When the droplets impact and then vaporize, the heat transfer between the rods and the coolant is improved. A model has been developed by Kendell and Rohsenow[61] to capture this effect and it has been implemented into CTF. This heat transfer effect is only applied for flow blockages caused by rod swelling in the local vicinity of the blockage; outside of this region, no droplet impingement heat transfer is felt by the rods.

Photographs taken of droplets impinging on an unquenched flat plate, heated above the Leidenfrost point, undergo a process of deformation, flattening, and reformulation[57][58]. The Kendell/Rohsenow model predicts this type of droplet behavior and the resulting evaporation efficiency. The droplets are viewed as cylindrical in shape and as they impact, the radius grows such that the droplet morphs into a large-radius, thin sheet of liquid. The predicted heat transfer efficiency due to droplet evaporation is then used in CTF to calculate the heat transfer between the rod and fluid. The heat transfer due to droplet evaporation is:

$$
Q=G_{e} h_{f g} \epsilon A_{z}
$$

$G_{e}$ is the mass flux of droplets in the blocked flow channel $\left(\mathrm{kg} / \mathrm{m}^{2} \mathrm{~s}\right), h_{f g}$ is the latent heat of vaporization, $\epsilon$ is the droplet evaporation efficiency (provided by the Kendell/Rohsenow model), and $A_{z}$ is the projected area normal to the flow. The user supplies the surface area of the rod portions that constitute the blockage along with the angle of the blockage with respect to the flow direction. This angle is used by CTF to determine the droplet velocity perpendicular to the rod surface. The Kendell/Rohsenow model for droplet heat transfer efficiency is given as follows:

$$
\begin{gathered}
\epsilon=2.6\left(\frac{\rho_{v}}{\rho_{l}}\right)^{1 / 2}\left(\frac{\bar{R}}{D}\right) W e_{p}^{1 / 8}\left[\frac{\beta_{2} k_{v}\left(T_{w}-T_{s}\right)}{h_{f g}\left(\rho_{v} \sigma g_{c} D\right)}\right]^{1 / 2} \\
{\left[0.225 \frac{\mu_{v} h_{f g}}{\beta_{2} k_{v}\left(T_{w}-T_{s}\right)}+1.5\right]^{-1 / 4}}
\end{gathered}
$$

The droplet Weber number, $W e_{p}$, is defined using the initial inertia of a spherical drop perpendicular to the wall.

$$
W e_{p}=\frac{\rho_{l} V_{p}^{2} D}{\sigma g_{c}}
$$

The velocity of the droplet, $V_{p}$, is the perpendicular velocity of the droplet with respect to the rod surface. The Weber number, in CTF, is set to a minimum of 30. The $\bar{R}$ term of Equation 6.9 is the average drop extension radius over the impact period and it is calculated as follows:

$$
\bar{R}=\frac{R_{\max }+0.43 D}{2}
$$


$R_{\max }$ is the maximum extension radius. This term is derived by equating the initial kinetic energy of the drop to the surface tension energy in the drop when it has come to complete rest in its new, flat-sheet form. This leads to the following equality.

$$
\frac{R_{\max }^{2}}{D^{2}}=\frac{2}{3}\left(1+\frac{W e_{p}}{12}\right) \cos ^{2}\left[\frac{1}{3}\left\{\arccos \left(\frac{-1.225}{\left(1+\frac{W e_{p}}{12}\right)^{3 / 2}}\right)\right\}\right]
$$

A special consideration is required here owing to the fact that the droplets are assumed to be a cylindrical geometry in the Kendell/Rohsenow model whereas the droplet Weber number is for a spherical droplet. Before deformation, the surface tension energies of a drop and cylinder are not equal if the volumes are equal and an initial amount of kinetic energy is required to account for this difference. Therefore, Equation 6.12 is only applicable for droplet Weber numbers greater than 1.74. For values less than this, it is assumed that $R_{\max }=0.43 D$, which is then consistent with Equations 6.11 and 6.9.

Finally, the $\beta_{2}$ term in Equation 6.9 is used to account for the nonlinear temperature profile in the vapor film between the droplet and the wall.

$$
\beta_{2}=\frac{1}{1+0.3 \frac{C_{p}\left(T_{w}-T_{s}\right)}{h_{f g}}}
$$

\subsubsection{Explicit Term Stability Criterion}

Since there are explicit terms in the heat conduction equation (if azimuthal or axial conduction are considered), there is also a stability criterion used in CTF. At the beginning of the conduction equation solution, the time step for the conduction equation is either set equal to or less than the flow solution time step. The user is responsible for providing CTF with a ratio that will be used to calculate the conduction equation time step with respect to the flow solution time step.

If axial or azimuthal conduction are modeled, CTF will calculate a maximum allowable timestep size for the heat conduction equation for each cell. The smallest of all the allowable timestep sizes for a single conductor (rod or wall) will be the limiting timestep size for the conduction equation for that particular conductor. For example, the limiting timestep, dtexp, is set equal to the initial heat conduction timestep when analysis starts on a conductor (it is re-initialized every time analysis moves to a new conductor). Then, if axial and azimuthal conduction are modeled, dtexp will be calculated for each cell after the explicit terms (axial and azimuthal conduction into or out of the current cell) are calculated. The stability criterion is basically a reformulation of the conduction equation written only for the axial and azimuthal conduction terms. The axial and azimuthal heat conduction from all adjacent cells is limited to the energy change in the current cell by solving for the time step. Specifically, the criterion is written as:

$$
\Delta t=\frac{\left(M C_{p}\right)_{(i, j, k)}}{K_{(i, j-1, k)}+K_{(i, j+1, k)}+K_{(i, j, k-1)}+K_{(i, j, k+1)}}
$$


This is the maximum allowable time step size for the given cell based on heat conduction from or to the adjacent cells. The limiting timestep for the entire conductor is found by taking the minimum of the $\Delta t$ for all the cells in the conductor. Prior to the implicit solution, the initial heat conduction timestep is compared to the minimum of the maximum allowable timesteps for all the cells. If the heat conduction timestep is greater than the smallest maximum allowable timestep, the heat conduction timestep is reduced by half or more. The reduction in the timestep size is dependent on the degree by which the conduction timestep exceeds the smallest maximum allowable timestep. This is done numerically in CTF by dividing the heat conduction timestep, dht, by the smallest maximum allowable timestep, dtexp. The conduction heat transfer timestep is then divided by one plus the integer portion of this result. Numerically, this is written as follows:

$$
d h t=\frac{d h t}{1+\operatorname{INT}\left(\frac{d h t}{d \operatorname{texp}}\right)}
$$

Here, the $I N T$ function returns the integer portion of the division. Therefore, if $d h t$ is at all greater than $d t e x p$, it will at least be reduced by half. If $d h t$ is twice as large than $d t e x p$, it will be reduced by a third, and so on. Clearly, it is necessary to use integer division so that the resulting number of conduction equation timesteps will fit into a single flow-field time step (i.e. at the end of conduction equation and flow-field equation solutions, the conductor and flow-field will be at the same time in the transient).

\subsection{Conductor Re-Meshing}

In a reactor core, during accident conditions, the special case of core re-wetting may occur. In this case, the liquid coolant that is introduced by safety injection systems re-fills the core and causes a quench front to form. At this quench front, where liquid coolant re-covers the once vapor-blanketed fuel rods and conductors, there are steep gradients in conductor/fluid heat flux and conductor temperatures. The behavior of the entire boiling curve - nucleate boiling, critical heat flux, transition boiling, and film boiling - may be experienced by the conductor/fluid interface in a relatively small locality. If this behavior is all captured by one, large conductor mesh cell as a single, averaged heat flux, the temperature through the conductor will not be correctly resolved. This is not only non-physical, but will also result in stepwise cell-by-cell quenching and will produce flow oscillations that will prevent the correct hydrodynamic solution from being obtained.

To remedy this problem, the capability to re-mesh the conductor in this vicinity has been implemented into CTF. Superimposing a finer mesh into the once coarse mesh of the quench front region allows the conductor temperature profile to be correctly resolved. The heat flux is solved for each refined conductor cell. The heat flux is then integrated over the refined cells to obtain the correct cell-averaged value to be applied to the adjacent fluid continuity mesh cell.

The initial meshing procedure in CTF for conductors is to create an axial conductor mesh cell for each adjacent axial fluid continuity mesh cell. An example is shown in Figure 6.6.

The conductor temperature and heat flux is calculated at the mesh cell center, shown in Figure 6.6 by black dots, just as the fluid pressure, enthalpy, and void fraction is solved at the cell centers. The axial length of the conductor and fluid cells are the same and the axial nodal locations of the conductor and fluid cells match up after initial meshing. The exception is for the top and bottom of the conductor where an extra conductor node is inserted. For the bottom conductor mesh cells, the cell height is $25 \%$ of the adjacent 


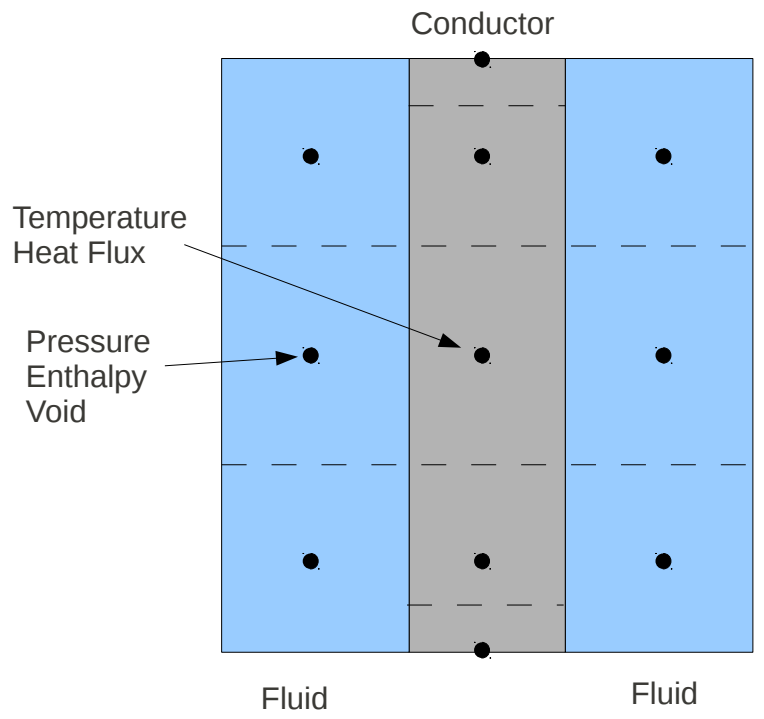

Figure 6.6: Example of how a conductor is meshed based on adjoining fluid mesh

fluid cell height - the cell height of the conductor mesh cell directly above the bottom cell is $75 \%$. In this manner, the top boundary of the second conductor mesh cell still meets with the boundary of the adjacent fluid mesh cell. Also, the second node is still at the same axial location as the adjacent fluid node. The meshing is performed in the same manner for the top conductor mesh cell.

When it is determined that re-meshing is necessary due to later-discussed criteria, a node is simply inserted halfway between pre-existing axial conductor nodes. This is shown in Figure 6.7 for the case of the first and second axial conductor cells which are $25 \%$ and $75 \%$ the height of the adjacent fluid cell, respectively.

When a cell is inserted, it is necessary to assign a temperature to that node. This must be done using an energy balance to ensure that energy is conserved in the conductor. This is shown as follows:

$$
\begin{aligned}
C p_{1}\left(T_{1}-T_{I}\right) \frac{\Delta X}{2}+C p_{2}\left(T_{2}-T_{I}\right) \frac{\Delta X}{2} & =0 \\
T_{I} & =\frac{(C p T)_{1}+(C p T)_{2}}{\left(C p_{1}+C p_{2}\right)}
\end{aligned}
$$

The subscripts 1 and 2 represent the original first and second cell values and the subscript $I$ represents the new inserted cell value. The benefit of the re-meshing procedure in CTF is that, not only does the code watch for zones that must be refined due to heat flux and temperature excursions, but it also watches for zones that can be coarsened when the quench front passes to other regions. If the mesh in a region has been refined, merging criteria will also be evaluated for that region to see when it is acceptable to coalesce the mesh cells back to form a coarser mesh. If the quench front no longer exists in a region that was refined, the 


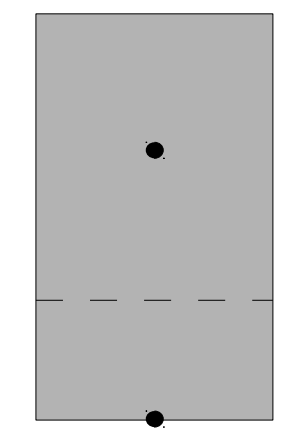

Before Refinement

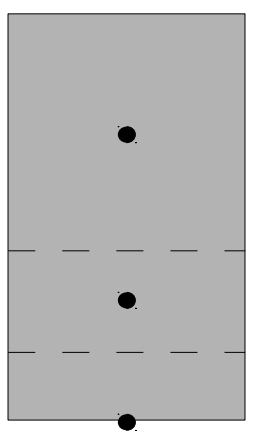

After Refinement

Figure 6.7: Example of conductor mesh cell refinement for the first and second cells of a conductor

mesh will eventually return to the original mesh that existed prior to refinement. This provides a benefit in computational resources.

The QFRONT subroutine is responsible for determining if a conductor cell should be split or if an alreadysplit cell should be coalesced. The QFRONT subroutine is called at the beginning of the HEAT subroutine prior to the determination of the heat transfer regime and associated heat transfer coefficients. QFRONT is called if there are any conductors being modeled by CTF. The QFRONT routine loops over all the rods in the model. For each rod, it then loops over each axial level of fluid cells adjacent the rod. For each axial fluid cell level, the routine loops over each conductor cell that is located within the bounds of the bottom and top of the adjacent fluid cell axial level (recall from Figures 6.6 and 6.7 that the boundaries of fluid cells always meet with conductor cell boundaries and that multiple conductor cells may exist for a single adjacent fluid cell). Finally, for each axial level of conductor cells in the rod, the routine loops over each surface of the rod.

It is within this loop that the routine checks criteria to determine whether to re-mesh or not. In this way, every surface cell of the rod is checked and axial re-meshing is performed when needed. QFRONT will then call the MOVE subroutine to perform the actual re-meshing process if it is deemed necessary for any surface in a given axial level of conductor cells. The criteria used to determine whether to split or merge refined cells are discussed in the following section.

\subsubsection{Re-Meshing Criteria}

The decision on whether a conductor cell should be split, coalesced with its neighbor cells, or left alone is a rather complicated one in CTF. Put most basically, the decision whether to re-mesh or not will be based on:

- Axial temperature gradient

- Local behavior of the heat transfer regimes 
- Pre-quench heat transfer differences in the axial direction

To best describe this procedure in as much detail as possible, it's best to start by presenting a flowchart of the decision process in all of its complexity. Next, we describe each step of the procedure, discussing the meaning of the terms and the condition that is evaluated. The procedure is roughly broken into two sections: assessing whether to coalesce the mesh and assessing whether to split (refine) the mesh. The flowchart blocks have been given unique identification numbers to make referencing them in text easier. Figure 6.8 presents the CTF procedure for deciding what to do with a conductor cell.

Before discussing the criteria, it is first important to make note that, for the case of mesh refinement, we are really interested in two conductor nodes. If certain criteria are not met (e.g. temperature and heat flux differences between those two conductor nodes are too high), the two cells will be split into three by inserting a node between them. When we consider whether to coalesce a cell (i.e. eliminate it), we are interested in three conductor nodes. If certain criteria are met (e.g. temperature differences between the first and third cells are not too high), the second conductor mesh cell may be eliminated and absorbed back into mesh cells 1 and 3 .

Coalescence Criteria Starting with Block 100, the first step is to check whether $\Delta X$, the conductor mesh cell axial height, is less than the user-specified minimum allowable conductor node size, $\Delta X_{\text {min }}$. The mesh cell will not be split any further if this is the case. Likewise, if the total number of axial heat conductor nodes in a rod exceeds the limit of 72 (Block 101), the conductor node will not be further split. Furthermore, the mesh cell will not be further split if the heat transfer regime (imode) is either single-phase vapor or singlephase liquid (Block 103). If any of these three blocks evaluate to true, CTF will check if the mesh cell should be coalesced or left alone.

Block 500 asks the question, is the conductor mesh cell (jr1) communicating with the same fluid cell that it was before re-meshing was performed $\left(j f\right.$ or $\left.j f_{+1}\right)$ ? If it is, then that means that the conductor mesh cell is the same as the original configuration and that no coalescence is needed; the mesh cell is left alone. If not, Block 501 checks if the last axial conductor node in the rod, nhtn, has been reached. If so, the analysis moves on to the next conductor. The current conductor is completed and no further checks are made on whether any conductor mesh cell in the current conductor should be split, merged, or left alone.

Following from this point (Block 502), a mesh cell will be coalesced into the conductor cells above and below if: (1) the heat transfer regime is either subcooled boiling or nucleate boiling and (2) if the resulting temperature difference between axial cells after merging takes place is small enough. For the first point, it's important to note that the imode definition of Block 502 differs from that of Blocks 102, 103, and 300. In Block 502, imode is the minimum of the heat transfer regime indices of conductor mesh cells 1 and 3 as opposed to mesh cells 1 and 2 .

As for Block 600, we define the meaning of the terms present. During this re-meshing process, we are really analyzing three conductor nodes: mesh cell 2 would be the inserted node (if re-meshing previously took place), mesh cell 1 would be the cell below the inserted node, and mesh cell 3 would be the cell above the inserted node. If the inserted node is coalesced, mesh cells 1 and 3 grow and take its place. So $\Delta T_{3}$ is defined in such a way that it captures the amount of temperature change over these three conductor cells. In equation form:

$$
\Delta T_{3}=\left|T_{2}-T_{1}\right|+\left|T_{2}-T_{3}\right|
$$




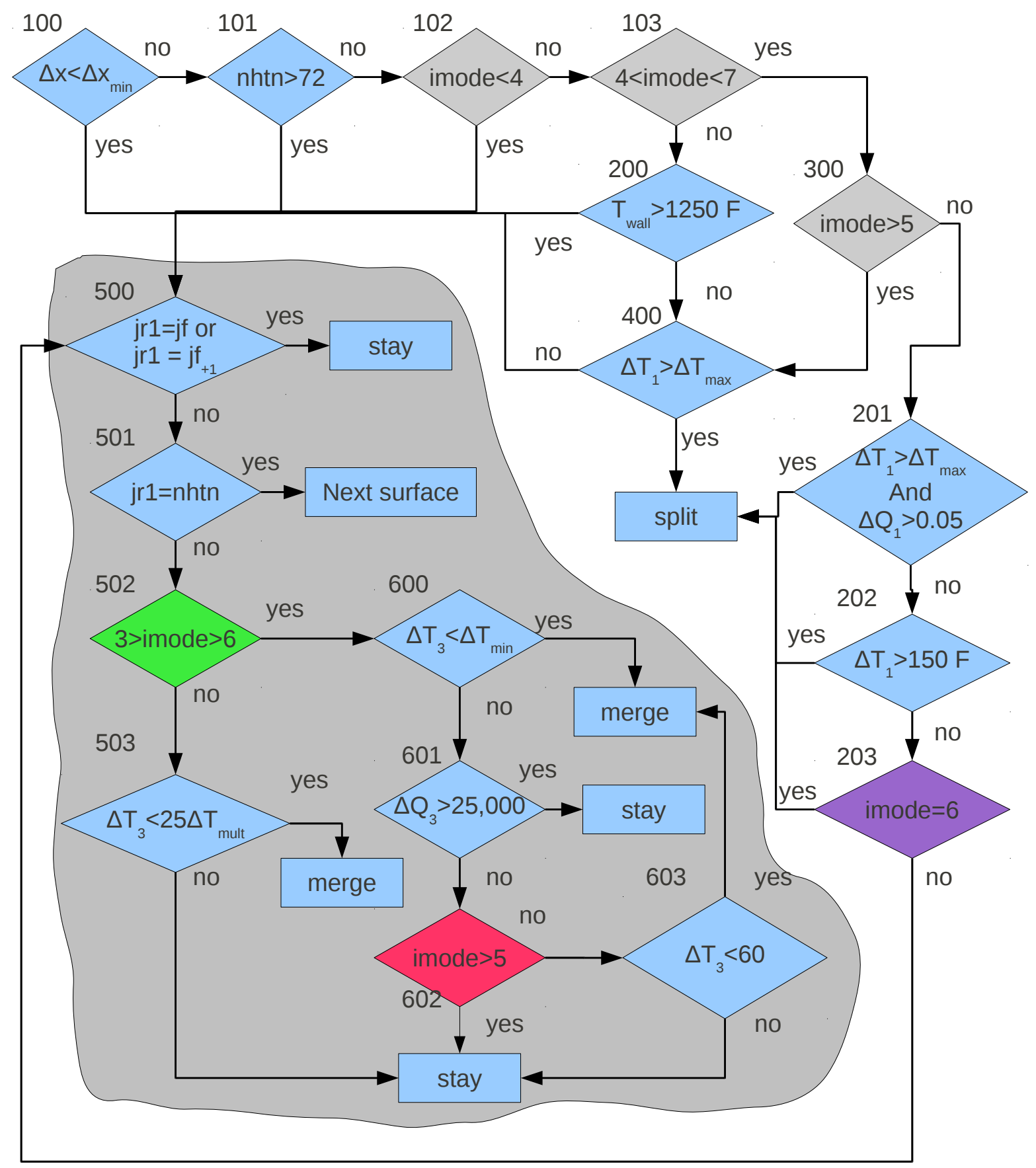

Figure 6.8: CTF procedure for determining whether conductor cells should be split, merged, or left alone 
$\Delta T_{\min }$ is the minimum temperature difference between conductor cells which will allow for their merging. This value is defined as a function of the rod surface temperature factor, $T_{w, \text { chf }}$.

$$
\Delta T_{\min }=\operatorname{dtmult}\left[2+\frac{1}{2} T_{w, \mathrm{chf}}^{2} * 15\right]
$$

dtmult is a multiplication factor that is set to 1 unless we are dealing with the top-most conductor cell that connects to the current fluid cell under analysis. In that case, the multiplier is set to 0.5 in order to cut the minimum temperature difference for coalescence in half. The criterion becomes more stringent because the conductor cells communicate with more than one fluid mesh cell in this case. $T_{w, \text { chf }}$ is a factor between 0 and 1 that captures how close to CHF the surface temperature is (surface temperatures close to the CHF temperature will produce a value near or equal to zero and surface temperatures far from the CHF temperature will produce values near or equal to 1 ). The factor is calculated by choosing the highest surface temperature, $T_{w}$ of the three conductor cells under analysis. Then, the following calculation is performed:

$$
T_{w, \mathrm{chf}}=\max \left(0, \min \left[1, \frac{T_{\mathrm{chf}}-T_{w}}{\max \left(T_{\mathrm{chf}}-T_{f}, 5\right)}\right]\right)
$$

If the surface temperature is close to $\mathrm{CHF}$, the allowable axial temperature difference will, correspondingly, also need to be small. However, for surface temperatures much smaller than CHF temperature, larger axial temperature differences are allowable. Barring a failure of Block 600 to be satisfied, there are still other means by which the inserted cell could be coalesced.

As shown in Block 601, having too large of a difference in heat flux requires the inserted cell to be left alone. If the difference in heat flux, $\Delta Q_{3}$, in the region of the three conductor cells being analyzed is greater than $25,000 \mathrm{BTU} / \mathrm{ft}^{2} \cdot \mathrm{hr}$, the conductor is not coalesced, but left alone. $\Delta Q_{3}$ is defined as $\Delta T_{3}$ was defined in Equation 6.17 in order to capture the changes in heat flux over all three of the conductor cells.

If the heat flux change in the three cells is small enough, Block 602 will be evaluated. If the heat transfer regime is either film boiling or dispersed flow boiling, it is necessary to keep the inserted node. Note again, that the definition of imode in Block 602 differs from all the other imode definitions in that it is the maximum of the indices of all three conductor nodes. So any of three conductor nodes may be in the transition or film boiling regime for the inserted cell to be left alone. Otherwise, there is one last criterion to check in Block 603. If the temperature change across the three conductor cells is less than $60^{\circ} \mathrm{F}$, the inserted cell can be coalesced, but if not, it must be left alone.

There is one remaining section of this portion of the flowchart that was neglected. Block 502 evaluated the heat transfer regime. If the heat transfer regime was either nucleate boiling or saturated boiling, evaluation of Block 600 commenced. However, if the regime isn't nucleate boiling or saturated boiling, CTF checks if the temperature difference in the three conductor cells is less than $25^{\circ} \mathrm{F}$ (Block 503). If this is true, then the inserted cell can be coalesced and if not, it must be left alone. This criterion is tightened to $12.4^{\circ} \mathrm{F}$ if the conductor cells communicate with more than one fluid mesh cell.

This portion of the flowchart discussed to this point covers all the criteria evaluated to determine if it is possible to coalesce the inserted cell. It is outlined in the grey section of Figure 6.8. 
Splitting Criteria To cover the remainder of the flowchart, we direct our attention to Block 103, having just established that the heat transfer regime is not single-phase vapor or liquid. At this point, imode was defined as the minimum of the heat transfer regime numbers for conductor mesh cells 1 and 2 . So if Block 103 evaluates to false, both conductor cells are experiencing some sort of film boiling. CTF checks if the surface temperature of both mesh cells 1 and 2 exceed $1250^{\circ} \mathrm{F}$. If they do, the inserted cell should be considered for coalescence. If one of these wall temperatures are less than this limit, the temperature difference between cells 1 and 2 are compared to the maximum allowable temperature difference. If this value is exceeded, then another cell must be inserted between the two in order to further refine the mesh. Otherwise, the inserted cell may be considered for coalescence. The maximum allowable temperature difference between cells is calculated as follows:

$$
\begin{aligned}
& \Delta T_{\max }=25^{\circ} \mathrm{F}+\text { dtwall } * 150^{\circ} \mathrm{F}, \text { where } \\
& d \text { twall }=\frac{1}{2}\left(1+\min \left[1, \max \left(0, \frac{T_{w}-T_{\min }}{1250^{\circ} \mathrm{F}-T_{\min }}\right)\right]\right)
\end{aligned}
$$

What this ends up boiling down to is that the higher the surface temperature in the film boiling regime, the larger the maximum allowable temperature difference will be.

Returning to Block 103, if the heat transfer regime is nucleate boiling or transition boiling, Block 300 is evaluated. If the heat transfer regime is transition boiling in one of the conductor cells, then the conductor cell temperature difference will be compared to the maximum allowable temperature difference (previously defined). Otherwise, the conductor cell temperature difference will still be compared to the maximum allowable temperature difference, but the heat flux difference between the two cells will also be limited. If the heat flux difference is greater than $50,000 \mathrm{BTU} / \mathrm{ft}^{2} \cdot \mathrm{hr}$, the mesh will be further refined.

Otherwise Block 202 is evaluated, where the mesh cell 1 and 2 temperature difference is compared to an upper limit of $150^{\circ} \mathrm{F}$. If that doesn't result in a mesh cell refinement, then a final criterion is checked. Block 203 re-defines the imode term to represent the heat transfer regime of the other conductor mesh cell. In other words, Block 203 asks if both conductor mesh cells are experiencing transition boiling. If they are, the mesh is refined and, if not, the mesh can be considered for coalescence.

\subsection{Nuclear Fuel Rod Models}

\subsubsection{Introduction}

It's important to predict the fuel centerline temperature in the reactor core accurately in terms of safe operation of the reactor core and its integrity below the fuel material melting point. CTF includes a package of special models for simulating the heat transfer behavior in nuclear fuel rods. The nuclear fuel rod model is suited specifically for $\mathrm{UO}_{2} / \mathrm{MOX}$ fuel and Zircaloy cladding. Fill gases composed of helium, xenon, argon, krypton, hydrogen, and nitrogen are accommodated. Material properties, like conductivity and specific heat, of the aforementioned metals and gases were taken from the MATPRO-11 handbook[62].

For a nuclear fuel rod with ceramic fuel pellets encased in a Zircaloy cladding, three regions are considered 
to be modeled:

1. The metal cladding with the Zircaloy cladding properties (Section 6.2)

2. The gap between the cladding and the fuel pellet. Thin gap approximation such that convection heat transfer can be neglected, therefore, conduction through the fill gas and radiative heat transfer between the pellet and cladding are modeled (Sections 6.4.3.1 and 6.4.3.3).

3. The fuel pellet. Under thermal treatments, the fuel pellets can expand/contract and may lead possible contacts between the pellet and cladding (Section 6.4.3.2). The change in fuel pellet dimensions has an effect on the gap size, attendantly, the gap heat transfer (Section 6.4.2).

A flowchart of the TEMP subroutine is given in Figure 6.9 with other subroutines called in. Firstly, conductivity and specific heat of the fuel pellet and cladding are determined by CTF before any subroutine calls. The fuel pellet conductivity is modified to account for cracking, if the fuel degradation model is chosen by the user and if cracking has occurred. After this, the GAPHTC array is called to obtain the gap heat transfer coefficient, accounting for radiative and conduction heat transfer. This subroutine calls the DEFORM subroutine to determine the new gap thickness and pellet and clad stresses. The GTHCON subroutine is called to calculate the actual gas conductivity, then, the heat transfer coefficient for the gap region by the GAPHTC subroutine. Following, the QOXIDE subroutine is called to account for the heat addition to cladding due to Zirconium/water reaction. Lastly, the GAUSS subroutine solves the set of conduction equations for the radial control volumes at a given axial location using Gaussian elimination and backwards substitution.

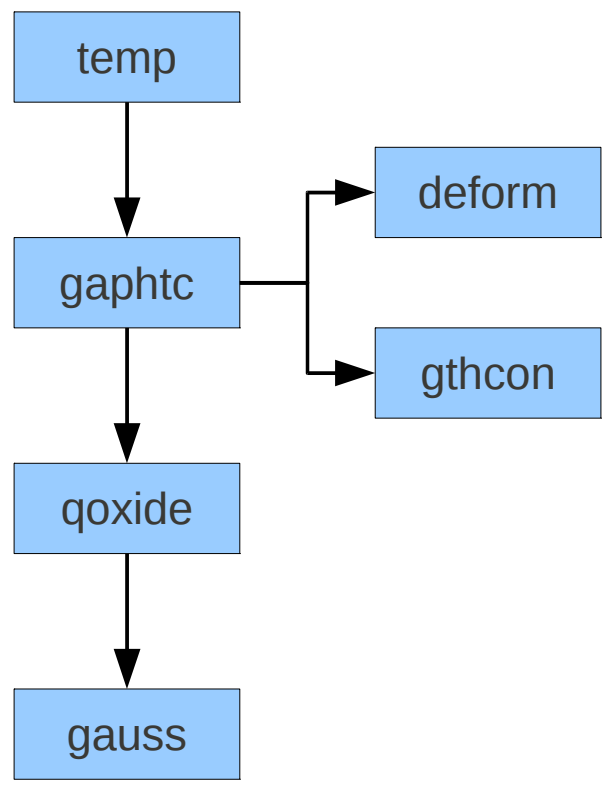

Figure 6.9: Flowchart of subroutine calls in the TEMP routine 
The following sections discuss this modeling process in detail. Determination of the gap thickness accounting for thermal expansion and pellet cracking is achieved with the fuel deformation model (Section 6.4.2). After this, the dynamic gap conductance model is discussed. There are three components of gap conductance: conduction through the gas (Section 6.4.3.1), radiative heat transfer (Section 6.4.3.3), and conduction through the pellet/clad interface (if contact exists) (Section 6.4.3.2). Finally, it has been mentioned that CTF also models heat generation in the cladding due to possible Zirconium-water reaction (Section 6.4.4).

\subsubsection{Fuel Deformation Model}

The fuel deformation model is encountered after the conductivity and specific heat material properties are defined for the pellet and cladding. Temperature driven expansion/contraction drives fuel rod deformation. Thermal expansion can directly cause fuel pellet and cladding geometry changes. Thermal expansion of the fuel pellet could also indirectly cause cladding deformation through mechanical stresses if the pellet and cladding come in contact (i.e. the expanding pellet could push on the cladding). The fuel pellet may also crack due to thermal expansion, which will cause relocation of the fuel inside the rod. This will, in turn, cause a change in fuel pellet conductivity as well as fuel pellet geometry. The fuel deformation model is used in two different places in CTF: the cracking effect on fuel pellet conductivity is considered in the TEMP subroutine, and the cracking and thermal/mechanical expansion effects on gap thickness are considered in the DEFORM subroutine. These are discussed in the following two sections.

Fuel Pellet Cracking and Sintering Effect on Conductivity When the fuel pellet expands and cracks, the conductivity is reduced due to the lower conductivity rod fill gas replacing the fuel pellet material in the cracks. An example of a cracked fuel pellet is shown in Figure 6.10.

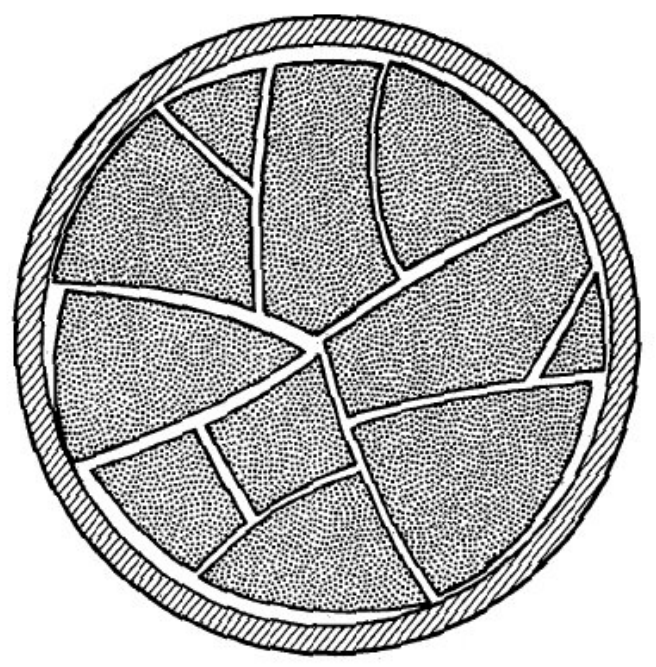

Figure 6.10: Illustration of fuel pellet cracking

The method for accounting for fuel thermal conductivity degradation was taken from the FRACAS-I me- 
chanics model[63]. The conductivity is changed by multiplication by a conductivity factor, $R$.

$$
K_{\text {eff }}=R K_{\text {lab }}
$$

The new, cracked fuel thermal conductivity, $K_{\text {eff }}$, is obtained by multiplying the conductivity factor by the uncracked fuel thermal conductivity, $K_{\text {lab }}$. The conductivity factor is a function of the gas conductivity and the volume available for cracking, which is given by

$$
R=1-0.3 \mathrm{~m}^{-1} C_{\mathrm{rel}}\left[1-\frac{k_{g}}{k_{\mathrm{lab}}}\right]
$$

The term $C_{\text {rel }}$ represents the volume that is available for cracking. It is the initial gap thickness, $\delta_{0}$, divided by $0.8 \cdot 10^{-4}$. Since cracked fuel will never fully solidify when compressed, $C_{\text {rel }}$ will never be less than 0.25 . The gas mixture conductivity, $k_{\mathrm{g}}$, is determined in the GTHCON subroutine and is discussed in Section 6.4.3.1. The uncracked fuel pellet conductivity, $k_{\text {lab }}$, is obtained from the $\mathrm{UO}_{2} / \mathrm{MOX}$ thermal conductivity correlation (see Section 9.1).

Crack healing is assumed to occur as the fuel temperature approaches the sintering temperature for $\mathrm{UO}_{2}$. The conductivity factor, $R$, is set to 1 when the fuel temperature exceeds $4304^{\circ} \mathrm{F}$, which is nine-tenths of the $\mathrm{UO}_{2}$ sintering temperature.

Fuel Pellet and Cladding Expansion Effects on Gap Thickness CTF accounts for thermal expansion of the fuel pellet and thermal/mechanical expansion of the fuel rod cladding in order to dynamically model the fuel rod gap thickness. Fuel pellet changes due to burnup-dependent quantities, such as swelling and densification, are not modeled. For an open gap (i.e. no pellet/clad contact), mechanical stress may be imposed on the cladding due to an imbalance between rod fill gas pressure and system operating pressure. For a closed gap, where the pellet and clad are in contact, expansion of the fuel pellet may impose a mechanical stress on the cladding. Other stresses and strains due to bending forces and creep deformation are ignored.

The phenomena affecting the gap thickness include: pellet thermal expansion $\left(\Delta r_{t h}\right)_{f u e l}$, clad thermal expansion $\left(\Delta r_{t h}\right)_{c l a d}$, fuel relocation due to cracking $\left(\Delta r_{r e l}\right)_{f u e l}$ and clad elastic expansion $\left(\Delta r_{e l}\right)_{c l a d}$. Each of these phenomena are further described. The components of the dynamic gap thickness, $\delta$, can be summarized into a single equation as:

$$
\delta=\delta_{0}-\left(\Delta r_{\text {th }}\right)_{\text {fuel }}+\left(\Delta r_{\text {th }}\right)_{\text {clad }}+\left(\Delta r_{\text {el }}\right)_{\text {clad }}-\left(\Delta r_{\text {rel }}\right)_{\text {fuel }}
$$

Fuel and Clad Thermal Expansion The fuel pellet radial and axial thermal expansion are calculated using the temperature-dependent fuel pellet thermal strain given by Equations 6.24 and 6.25. The strain is multiplied by the radial thickness of the control volume to calculate radial expansion $\left(\Delta r_{\text {th }}\right)_{f u e l}$ and by the axial height of the control volume to calculate axial expansion $\left(\Delta l_{t h}\right)_{f u e l}$. For the axial expansion, the temperature used is the average temperature for the axial level under analysis, obtained by volume averaging of the radial control volume temperatures. Thermal expansion is calculated for each control volume and then the total pellet expansion is calculated simply by summing the expansion of all control volumes in the pellet. 
In the following equations, NFUEL and NDX represent the total number of radial nodes in the fuel and total number of axial nodes in the fuel, respectively.

$$
\begin{gathered}
\left(\Delta r_{\mathrm{th}}\right)_{\text {fuel }}=\Sigma_{i=1}^{\mathrm{NFUEL}} \epsilon_{\mathrm{UO}_{2}}\left(T_{i j}\right) \Delta r_{i} \\
\left(\Delta l_{\mathrm{th}}\right)_{\text {fuel }}=\Sigma_{j=1}^{\mathrm{NDX}} \epsilon_{\mathrm{UO}_{2}}\left(\bar{T}_{j}\right) \Delta X_{j}
\end{gathered}
$$

The cladding expansion is similarly calculated; however, separate strain correlations exist for the axial and radial directions for Zircaloy (Section 9.2). Furthermore, only one control volume is used in the radial direction for the cladding, so radial expansion only needs to multiply by the clad mean radius, $\bar{r}$.

$$
\left(\Delta r_{\text {th }}\right)_{\text {clad }}=\epsilon_{r}\left(\bar{T}_{j}\right) \bar{r}
$$

The averaged cladding temperature at the given axial level is used. Axial expansion of the clad considers all axial nodes, NDX, in the clad.

$$
\left(\Delta l_{\mathrm{th}}\right)_{\mathrm{clad}}=\Sigma_{j=1}^{\mathrm{NDX}} \epsilon_{Z}\left(T_{j}\right) \Delta X_{j}
$$

Fuel Pellet Relocation The fuel pellet geometry changes due to thermal expansion. The fuel pellet may also crack, causing it to creep further into the pellet/clad gap and even contact the clad. The relocation model to account for this cracking-induced movement of the fuel pellet is the FRACAS-I mechanics model[63]. The amount of pellet relocation in an open gap was expressed as a function of cold-state rod geometry by Coleman[64].

$$
\left(\Delta r_{\text {rel }}\right)_{\text {fuel }}=\delta_{0}-0.005 r_{f}
$$

Dimensions are in $\mathrm{ft}, \delta_{0}$ is the as-fabricated fuel/clad gap size, and $r_{f}$ is the cold-state radius of the fuel pellet. $\Delta r$ is the amount by which the fuel pellet radius changes, which will remain constant so long as the gap remains open (i.e. no pellet/clad contact). The gap is considered to be closed when the gap thickness decreases to less than 1.8 (or user provided value for factor_gapcriterion) times the sum of the clad and pellet surface roughness. If the gap is closed, fuel relocation is modified to be large enough to keep the gap closed - fuel relocation will not cause mechanical expansion of the cladding. This is written in CTF as:

$$
\left(\Delta r_{\text {rel }}\right)_{\text {fuel }}=\max \left[0, \delta-1.8\left(\epsilon_{\text {fuel }}+\epsilon_{\text {clad }}\right)+\left(\Delta r_{\text {rel }}\right)_{\text {fuel }}\right]
$$

Here, $\epsilon_{\text {fuel }}$ and $\epsilon_{\text {clad }}$ represent the pellet and clad surface roughness, in $\mathrm{ft}$, respectively. The expression ensures that the amount of pellet relocation due to cracking is no bigger than the gap thickness. 
Cladding Elastic Deformation The cause of cladding elastic deformation depends on the state of the gap. If the gap is open, cladding stresses will be caused by a differential in fill gas pressure and reactor system pressure. If the gap is closed, cladding stresses will be caused by fuel pellet thermal expansion. For both cases, the cladding is assumed sufficiently thin that stress, strain, and temperature can be considered uniform throughout the cladding thickness.

The concept, for either an open or closed gap, is to calculate the clad stress and, using Hook's Law, obtain the radial displacement of the cladding. The hoop stress $\sigma_{\theta}$ and axial stress $\sigma_{Z}$ that exist in the cladding - both are functions of the cladding inside and outside pressure - are defined as:

$$
\begin{gathered}
\sigma_{\theta}=\frac{r_{i} P_{i}-r_{o} P_{o}}{t_{c}} \\
\sigma_{Z}=\frac{\pi r_{i}^{2} P_{i}-\pi r_{o}^{2} P_{o}}{\pi\left(r_{o}^{2}-r_{i}^{2}\right)}
\end{gathered}
$$

The $i$ and $o$ subscripts represent the inner and outer faces of the cladding and the cladding thickness, $t_{c}$, accounts for changes in cladding dimensions due to expansion. With the hoop and axial stresses calculated, it's possible to use Hook's Law to convert these values to cladding displacement using Young's modulus and the shear modulus (see Section 9.2).

$$
\begin{gathered}
\epsilon_{\theta}=\frac{\Delta r}{\bar{r}}=\frac{1}{E}\left(\sigma_{\theta}-\nu \sigma_{Z}\right) \\
\epsilon_{Z}=\frac{\Delta l}{l}=\frac{1}{E}\left(\sigma_{Z}-\nu \sigma_{\theta}\right)
\end{gathered}
$$

The radial deformation of the cladding can simply be calculated by multiplying the mean cladding radius, $\bar{r}$, by hoop strain.

$$
\left(\Delta r_{\mathrm{el}}\right)_{\mathrm{clad}}=\epsilon_{\theta} \bar{r}
$$

The axial deformation is calculated by summing the axial expansion of each control volume in the axial direction.

$$
\left(\Delta l_{\mathrm{el}}\right)_{\mathrm{clad}}=\Sigma_{j=1}^{\mathrm{NDX}} \epsilon_{Z} \Delta X_{j}
$$

The distinction between cladding deformation caused by an open gap and a closed gap lies in the cladding interior pressure used to calculate the cladding stresses in Equations 6.30 and 6.31. For an open gap, the fill gas pressure, $P_{g}$, is substituted for $P_{i}$. The fill gas pressure is from the previous time step since fill gas pressure is calculated at the end of the deformation model. It is calculated using a static lumped pressure model similar to those in FRAP and GAPCON. The pressure is assumed uniform throughout the fuel pin with constant fission gas inventory. 


$$
P_{g}=\frac{M R}{\frac{V_{p}}{T_{p}}+\Sigma_{j=1}^{N D X} \pi \Delta X_{j}\left[\frac{\left(r_{c i}^{2}-r_{f o}^{2}\right)}{T_{g}}+\frac{r_{v}^{2}}{T_{v}}+\frac{\left(r_{f o}^{2}-r_{f}^{2}\right)}{T_{F}}\right]_{j}}
$$

Terms are defined as follows:

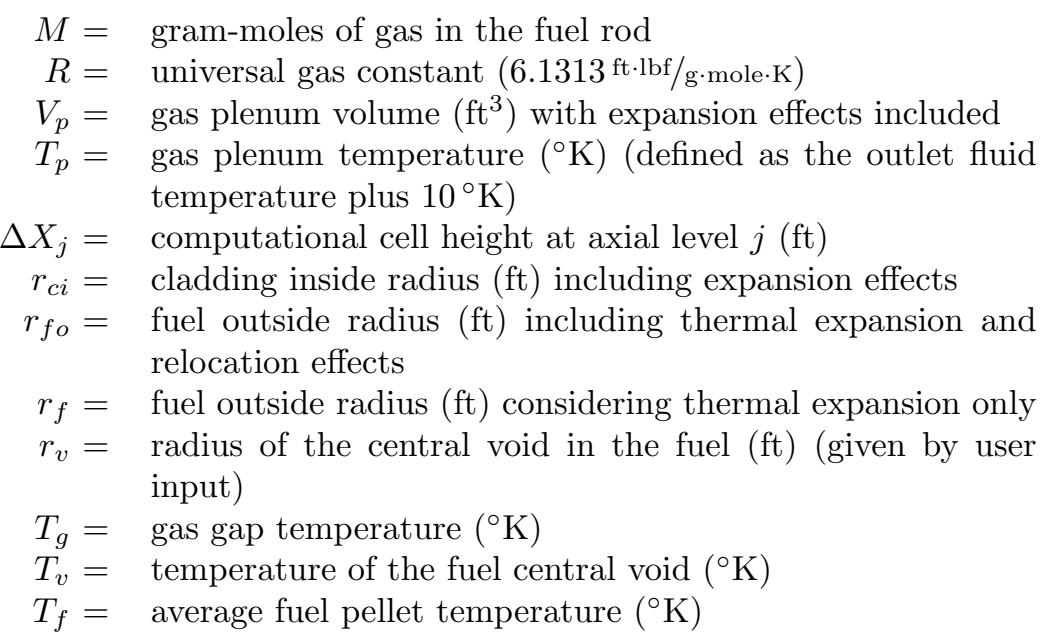

For the closed gap scenario, the interface pressure, $P_{\text {int }}$, is used as the inner clad surface pressure in Equations 6.30 and 6.31. The interface pressure is caused by radial displacement of the fuel due to thermal expansion which, in turn, causes radial displacement of the cladding. The cladding radial displacement is a combination of fuel rod thermal expansion, relocation, and cladding thermal expansion. Cladding radial displacement is defined as:

$$
\Delta r_{\text {clad }}=1.8\left(\epsilon_{\text {fuel }}+\epsilon_{\text {clad }}\right)-\delta_{0}+\left(\Delta r_{\text {th }}\right)_{\text {fuel }}-\left(\Delta r_{\text {th }}\right)_{\text {clad }}+\left(\Delta r_{\text {rel }}\right)_{\text {fuel }}
$$

The first term on the RHS defines the closed gap width accounting for the surface roughness of the cladding and fuel. Followed by this term are the cold-state gap width, fuel thermal expansion, cladding thermal expansion, and fuel relocation due to cracking. This amount of cladding displacement can be substituted into the hoop strain equation (Equation 6.32) which is the function of the hoop stress $\sigma_{\theta}$ and axial stress $\sigma_{Z}$. The stress terms are functions of the interior pressure, $P_{i}$ which is actually the interface pressure, $P_{\text {int }}$. Consequently, a single equation can be formed for interface pressure as:

$$
P_{\text {int }}=\frac{\Delta r_{\text {clad }} E t_{c}\left(r_{o}^{2}-r_{i}^{2}\right)}{\bar{r}\left[r_{i}\left(r_{o}^{2}-r_{i}^{2}\right)-r_{i}^{2} t_{c} \nu\right]}+P_{o} \frac{r_{o}\left(r_{o}^{2}-r_{i}^{2}\right)-r_{o}^{2} t_{c} \nu}{r_{i}\left(r_{o}^{2}-r_{i}^{2}\right)-r_{i}^{2} t_{c} \nu}
$$

Note that cladding dimensions do not take into account any previously calculated elastic and thermal expansion. 


\subsubsection{Dynamic Gap Conductance Model}

The gap conductance (or total conductance across the gap) is defined in the dynamic gap conductance model as

$$
H_{\text {gap }}=H_{\text {gas }}+H_{\text {contact }}+H_{\text {rad }}
$$

in which the conduction in the gap is composed of three components; conduction through the fill gas $H_{g a s}$, solid-solid contact conductance $H_{\text {solid }}$, and radiative heat transfer in the gap $H_{\text {rad }}$. It is assumed that the gap between the fuel rod and the cladding is thin enough such that there is no convection heat transfer caused by the gas, therefore, conduction heat transfer will be dominant heat transfer.

\subsubsection{Conduction Through the Fill Gas in the Gap}

Conduction heat transfer through the fill gas will exist whether the gap is open or closed due to fuel expansion, but the calculation of heat conduction in the fill gas in a closed gap is slightly different than for that of an open gap. The model used for calculating gap fill gas heat conduction was taken from GAPCON-2, which was based on a linear regression analysis of Ross-Stoude data by Lanning and Hann[65]. For either case, the heat conduction is defined as the gas mixture conductivity divided by the gap thickness.

$$
H_{\text {gas }}=\frac{k_{\text {gas }}}{\delta_{\text {gas }}}
$$

The gap thickness, however, takes account for the fact that the temperature gradient in the fill gas is not a linear one. Instead, there is a jump in temperature near the pellet and clad surface due to incomplete thermal mixing of the gas molecules in that localized region. This concept is illustrated in Figure 6.11. To account for this discontinuity, a temperature jump distance is added to the physical gap thickness. Accounting for the temperature jump distances, $g_{1}$ and $g_{2}$, the heat conduction is defined as:

$$
H_{\mathrm{gas}}=\frac{k_{\mathrm{gas}}}{t_{g}+1.845\left(g_{1}+g_{2}\right)}
$$

where the gas conductivity is given in units of BTU/hr.ft.F and the physical gap thickness, $t_{g}$, and jump distance units are in $\mathrm{ft}$.

For a closed gap, there are still regions of fill gas where conduction takes place due to the surface roughness of the pellet and clad. A gap is considered closed when the gap thickness is less than 1.8 times the sum of the surface roughnesses. The gap thickness for a closed gap is taken as half of this value times a modification factor that takes into account the contact pressure between cladding and gap, which was previously defined in Section 6.4.2. The jump distances in the gap are still considered. Equation 6.40, for a closed gap, is written:

$$
H_{\text {gas }}=\frac{k_{\text {gas }}}{1.8\left(C\left(R_{1}+R_{2}\right)+\left(g_{1}+g_{2}\right)\right)-4.2 \cdot 10^{-7}}
$$

The surface roughness of fuel and clad are given by $R_{1}$ and $R_{2}$ in $\mathrm{ft}$. For numerical stability, a lower limit of $1 \cdot 10^{-6}$ is imposed for the denominator. The dimensionless modification factor that account for interface pressure, $P_{\text {int }}$, is given as $C$ in the above equation. It is defined as:

$$
C=1.98 \exp -8.8 \cdot 10^{-5} P_{\text {int }}
$$




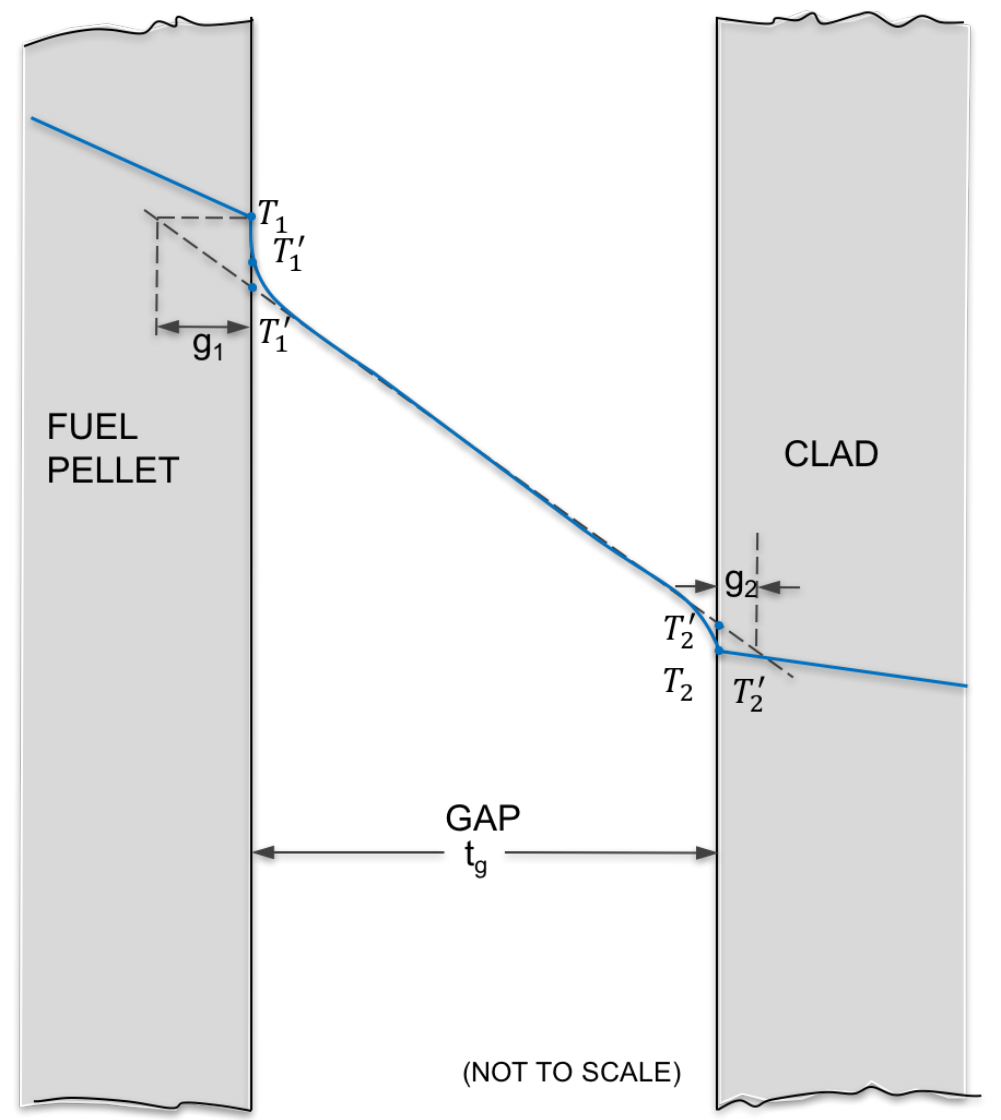

Figure 6.11: Illustration of the temperature jump near fuel pellet and clad surfaces in a fuel rod gap 
For both the open and closed gap models, it is necessary to define the gap gas conductivity and the surface temperature jump distances, which appear in the above heat conduction equations.

Fill Gas Thermal Conductivity The thermal conductivity for a mixture of gases is calculated considering the individual gas conductivities (see Section 9.3) as well as their molecular weights and mole fractions. The gas mixture thermal conductivity (BTU/hr-ft-F) is

$$
\begin{aligned}
k_{\mathrm{gas}} & =\Sigma_{i=1}^{N} \frac{k_{i}}{1+\sum_{\substack{j=1 \\
i \neq j}}^{N} \Psi_{i j} \frac{X_{j}}{X_{i}}} \\
\Psi_{i j} & =\phi_{i j}\left(1+2.41 \frac{\left(M_{i}-M_{j}\right)\left(M_{i}-0.142 M_{j}\right)}{\left(M_{i}+M_{j}\right)^{2}}\right) \\
\phi_{i j} & =\frac{\left[1+\left(\frac{k_{i}}{k_{j}}\right)^{1 / 2}\left(\frac{M_{i}}{M_{j}}\right)^{1 / 4}\right]^{2}}{2^{3 / 2}\left(1+\frac{M_{i}}{M_{j}}\right)^{1 / 2}}
\end{aligned}
$$

where the summations are performed over all gases in the mixture, where $N$ is the total number of gases (maximum of 6 ). $X$ and $M$ are the mole fraction and molecular weights of gases, respectively.

Temperature Jump Distance The temperature jump distance is calculated from the GAPCON-2 modification of the Lloyd model[66], and used in CTF as:

$$
\left(g_{1}+g_{2}\right)=2.0358 \cdot 10^{-5} \frac{k_{\mathrm{gas}} \sqrt{\bar{T}_{\mathrm{gas}}}}{P_{\mathrm{gas}} \Sigma_{j=1}^{N} \frac{a_{j} f_{f}}{\sqrt{M_{j}}}}
$$

where $k_{\text {gas }}$ is fill gas thermal conductivity $(\mathrm{BTU} / \mathrm{hr}-\mathrm{ft}-\mathrm{F}), \bar{T}_{\text {gas }}$ is mean gas gap temperature $\left({ }^{\circ} \mathrm{K}\right)$ and $P_{\text {gas }}$ is the fill gas pressure (psia). $f_{j}, a_{j}$ and $M_{j}$ are the mole fraction, the accommodation coefficient and the molecular weight of gas $\mathrm{j}$, respectively.

The summation in the denominator is performed over all individual fill gases in the rod (total of 6 ). There is a temperature dependent accommodation factor for each gas. Ullman[67] made measurements for helium and xenon on $\mathrm{UO}_{2}$ and curve fits were made to these data for use in GAPCON-2. These curve fits were implemented into CTF, and are given below:

$$
\begin{aligned}
& a_{\mathrm{He}}=0.425-2.3 \cdot 10^{-4} T_{\circ} K \\
& a_{\mathrm{Xe}}=0.749-2.5 \cdot 10^{-4} T_{\circ} K
\end{aligned}
$$

and the linear interpolation is simply applied for the other gases that have molecular weights between those of helium and xenon as:

$$
a_{j}=\frac{a_{\mathrm{Xe}}-a_{\mathrm{He}}}{128} M_{j}+\left(a_{\mathrm{He}}-4 \frac{a_{\mathrm{Xe}}-a_{\mathrm{He}}}{128}\right)
$$


This was found to correlate with the data of Thomas[68] with reasonable accuracy.

\subsubsection{Conduction in the Gap due to Pellet/Clad Contact}

The solid contact conduction term will only be nonzero if the clad and pellet are found to be in contact. When contact exists, the Mikic/Todreas model[69][70] is used to predict associated heat transfer.

$$
H_{\text {solid }}=\frac{5 k_{m}}{\sqrt{R_{f}^{2}+R_{c}^{2}}}\left(\frac{P_{\mathrm{int}}}{H_{Z}}\right)^{n}\left(\frac{R_{f}}{\lambda_{f}}\right)
$$

The interface pressure, $P_{\text {int }}$, was discussed as part of the fuel deformation model (Section 6.4.2). The Meyer hardness of Zircaloy, $H_{Z}$, is defined as a temperature dependent correlation in Section 9.2. The roughness parameters, $R_{f}$ and $R_{c}$, should be entered in units of inches. The conductivity at the interface is a function of fuel and cladding conductivities, defined as:

$$
k_{m}=\frac{2 k_{f} k_{c}}{k_{f}+k_{c}}
$$

The nondimensionalized interface pressure, $P_{\text {int }} / H_{Z}$, has an exponent, $n$, associated with it. The exponent is selected based on the value of the nondimensionalized interface pressure as:

$$
n=\left\{\begin{array}{lll}
0.5 & \text { for } & P_{\text {int }} / H_{Z}<0.0001 \\
1.0 & \text { for } & P_{\text {int }} / H_{Z}>0.01
\end{array}\right.
$$

and for values of $0.001<P_{\mathrm{int}} / H_{Z}<0.01$, the entire expression, $\left(P_{\mathrm{int}} / H_{Z}\right)^{n}$, is held to a constant of 0.01 .

The final expression of Equation 6.48 is the dimensionless ratio of mean fuel surface roughness to fuel wave length, $R_{f} / \lambda_{f}$. This expression is estimated in GAPCON-2 by the equation:

$$
\frac{R_{f}}{\lambda_{f}}=\exp \left[0.5285 \ln \left(R_{f}-5.738\right)\right]
$$

It's important to note, here, that the fuel surface roughness in the expression must be entered in microinches.

\subsubsection{Radiative Heat Transfer in the Gap}

The radiation heat transfer in the gap is defined as the radiation heat flux divided by the gap temperature gradient.

$$
H_{\mathrm{rad}}=\frac{q_{r}^{\prime \prime}}{T_{f}-T_{c}}
$$

Subscripts $f$ and $c$ stand for "fuel" and "cladding". The radiant heat flux is determined from the StefanBoltzmann equation. 


$$
q_{r}^{\prime \prime}=\sigma_{S B} \frac{T_{f}^{4}-T_{c}^{4}}{\frac{1}{\epsilon_{f}}+\frac{A_{f}}{A_{c}}\left(\frac{1}{\epsilon_{c}-1}\right)}
$$

Temperature is provided in units of Rankine. The Stefan-Boltzmann constant, $\sigma_{S B}$, is $1.714 \cdot 10^{-9} \mathrm{BTU} / \mathrm{hr}^{-\mathrm{ft}^{2}} \cdot \mathrm{R}^{4}$. Uranium dioxide and Zircaloy emissivities are given in Sections 9.1 and 9.2.

\subsubsection{Metal/Water Reaction}

For nuclear fuel rods with a zirconium-based cladding, there can be an exothermic reaction between the zirconium and water or steam at high temperatures.

$$
2 \mathrm{H}_{2} \mathrm{O}+\mathrm{Zr} \rightarrow 2 \mathrm{H}_{2}+\mathrm{ZrO}_{2}+140500 \mathrm{cal} / \mathrm{mole} \mathrm{Zr}
$$

According to work done by Cathcart and Pawel[71], the oxidation rate of zirconium at temperatures above $1000^{\circ} \mathrm{C}$ follows a parabolic rate law.

$$
W \frac{d W}{d t}=A \exp \left(\frac{-B}{R T}\right)
$$

The terms are:

$$
\begin{aligned}
W & =\text { total oxygen consumed }\left(\mathrm{kg} / \mathrm{m}^{2}\right) \\
A & =16.8 \mathrm{~kg}^{2} / \mathrm{m}^{4} \mathrm{~s} \\
B & =\text { activation energy, } 39940 \mathrm{cal} / \mathrm{gm} \cdot \mathrm{mole} \\
R & =\text { gas constant, } 1.987 \mathrm{cal} / \mathrm{gm} \cdot \mathrm{mole} \cdot{ }^{\circ} \mathrm{K} \\
T & =\text { temperature }\left({ }^{\circ} \mathrm{K}\right)
\end{aligned}
$$

In CTF, we are interested in two things: the thickness of the oxide layer and the heat generation in the cladding due to oxidation. Equation 6.55 tells us of the amount of oxygen consumed by the cladding in units of mass per time. By dividing this equation by the density of zirconium, we can obtain the change in the oxide layer thickness over time.

$$
\tau \frac{d \tau}{d t}=\frac{A}{\rho^{2}} \exp \left(\frac{-B}{R T}\right)
$$

The oxide layer thickness is expressed here as $\tau$. To determine the change in oxide layer thickness over a single time step, we integrate this equation over the time step size and from the old time step oxide layer thickness to the new time oxide layer thickness. 


$$
\int_{\tau_{0}}^{\tau} \tau d \tau=\frac{A}{\rho^{2}} \exp \left(\frac{-B}{R T}\right) \int_{0}^{\Delta t} d t
$$

This leads to:

$$
\begin{aligned}
\frac{1}{2}\left(\tau^{2}-\tau_{0}^{2}\right) & =\frac{A}{\rho^{2}} \exp \left(\frac{-B}{R T}\right) \Delta t \\
\tau^{2} & =\frac{2 A}{\rho^{2}} \exp \left(\frac{-B}{R T}\right) \Delta t+\tau_{0}^{2}
\end{aligned}
$$

The oxide layer radius is defined as the distance from the fuel centerline to the inner surface of the oxide layer in CTF, so this thickness is subtracted from the outer radius of the fuel rod to obtain the new oxide layer radius. In CTF, $R$ is absorbed into $B$ in the exp function, leading to a coefficient of $20070{ }^{\circ} \mathrm{K}$. Using a zirconium density of $691.8 \mathrm{~kg} / \mathrm{m}^{3}$ for the operating temperature and pressure leads to a leading coefficient of $7.02 \cdot 10^{-5} \mathrm{~m}^{2} / \mathrm{s}$. The energy generated in the cladding is the second item of interest. This is calculated by multiplying the energy caused by oxidation by the amount of zirconium oxidized in a timestep.

$$
q_{o}=Q_{c} \frac{V}{\Delta t}
$$

The energy generation, $q_{o}$, is a rate term in units of $\mathrm{J} / \mathrm{s}$. The energy released by oxidation, $Q_{c}$, is 4.044 . $10^{9} \mathrm{~J} / \mathrm{m}^{3}$. Expanding out the volume term, $V$, leads to:

$$
q_{o}=Q_{c} \frac{\pi\left(r_{\text {oxide }, \text { old }}^{2}-r_{\text {oxide,new }}^{2}\right) \Delta X}{\Delta t}
$$

The $\Delta X$ term is the axial height of the conductor mesh cell.

\subsection{Departure from Nucleate Boiling (DNB)}

DNB is the point at the top of the boiling curve, shown in Figure 6.12. It is the point where vapor blanketing of the heated surface begins to decrease heat transfer from the surface to fluid with increasing temperature. Operating the rod in post-CHF heat transfer regimes can lead to high temperatures and subsequent rod failure.

The calculation of DNB is required to properly assign the heat transfer regime in CTF. The determination of the heat transfer regime is made by comparing the local heat flux to the CHF. CHF is the heat flux at which DNB occurs. In practice, CTF actually determines a temperature at which $\mathrm{CHF}$ occurs $\left(T_{\text {chf }}\right)$ and then compares the local rod surface temperature to that value; if the local temperature is above $T_{\text {chf }}$, the heat transfer regime changes to a post-CHF heat transfer regime. Otherwise, the heat transfer regime will be pre-CHF. 


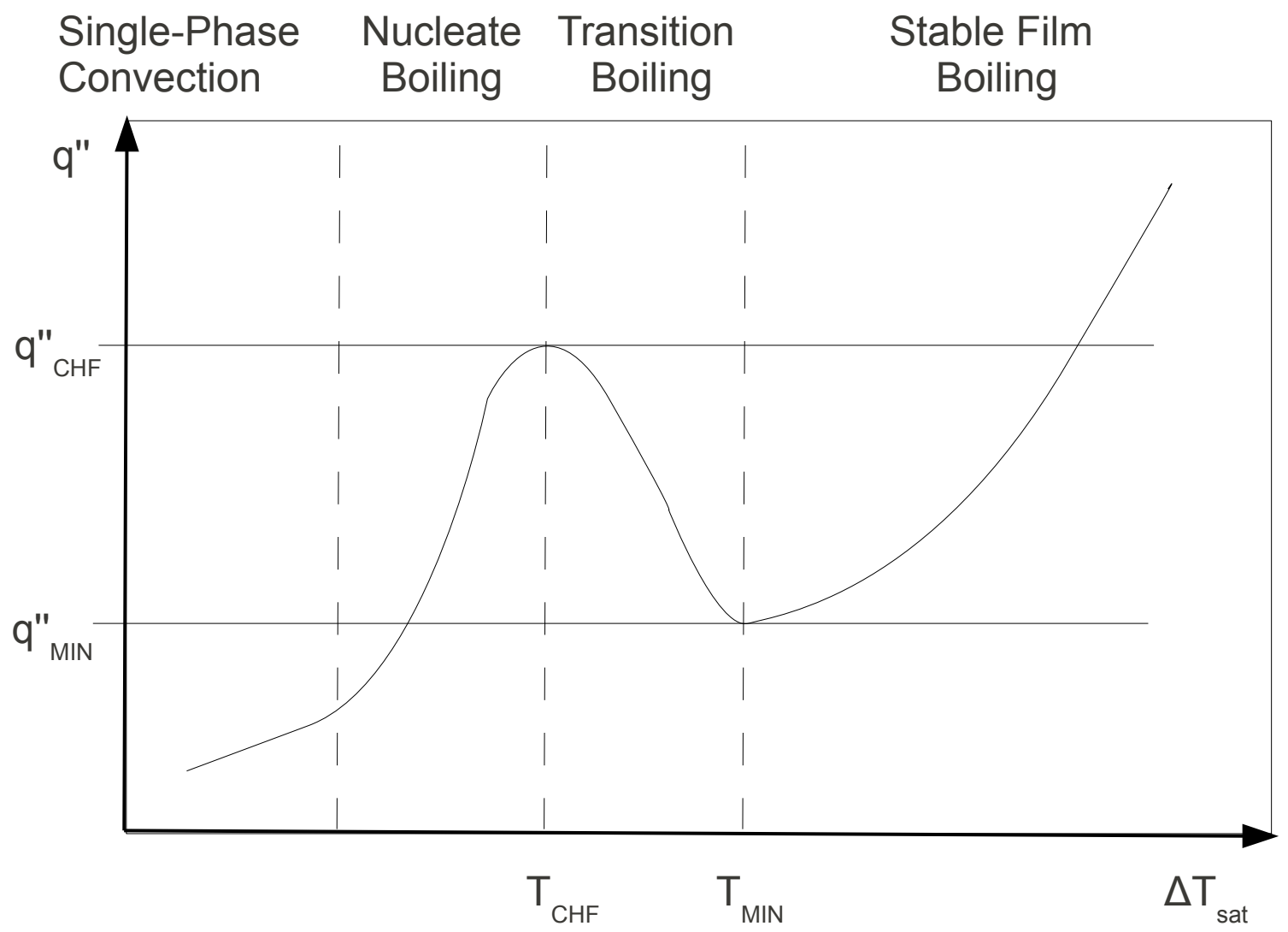

Figure 6.12: Boiling Curve 
Calculating the CHF is the first step in this process. The local value of CHF is calculated using one of many available models in $\mathrm{CTF}$. After $\mathrm{CHF}$ is calculated, $T_{\text {chf }}$ is determined using an iterative process that is described in Section 6.5.6.

\subsubsection{No Model}

It is possible to use no model. In this case, the code will skip the CHF calculation and assume that the heat transfer regime is always pre-CHF. This feature should only be used when the user is certain CHF will not be encountered. If the user is sure of this, some savings can be made in computational time.

\subsubsection{Biasi Model}

An overview of the CHF calculation algorithm for the Biasi model is shown in Figure 6.13. The standard model consists of considering three possible CHF scenarios:

\section{Pool boiling \\ 2. Forced convective boiling \\ 3. Annular film dryout}

If the mesh cell was labeled as having a hot wall in the INTFR subroutine, then CHF is calculated for the annular film dryout region. If the mesh cell does not contain a hot wall, the void fraction is checked because the boiling regime could still be in annular film dryout. If $\alpha_{v}>0.9$, the annular film dryout CHF is calculated and if $\alpha_{v}>0.99$, the annular film dryout CHF is multiplied by a ramping factor to reduce CHF down to $20 \%$ of its calculated value as $\alpha_{l}$ approaches 0.005 .

If $\alpha_{v}$ was less than 0.9, then CHF is calculated for the forced-convection boiling regime. If the mass flux is less than $30 \mathrm{~g} / \mathrm{cm}^{2} \cdot \mathrm{s}$, then the CHF is ramped to CHF for the pool boiling scenario - the maximum of either the forced-convection CHF or the annular film dryout CHF multiplied by a ramping factor to account for low liquid void is chosen.

Whether forced-convection or pool boiling is selected, a check is made for void over 0.85 and, if so, the CHF is ramped towards annular film dryout CHF as shown in Equation 6.61. The heat flux, $q^{\prime \prime}$ in Equation 6.61 could be either pool boiling CHF or forced-convection CHF depending on the flow conditions.

$$
f\left(q_{\mathrm{AF}}^{\prime \prime}, q^{\prime \prime}\right)=20\left[q^{\prime \prime}\left(\alpha_{l}-0.1\right)+q_{\mathrm{AF}}^{\prime \prime}\left(0.15-\alpha_{l}\right)\right]
$$

For any of the three scenarios, the CHF is ramped towards the single phase vapor heat transfer coefficient times a $\Delta T$ of $50^{\circ} \mathrm{F}$ if $\alpha_{v}$ is greater than 0.99 . The ramp is as follows:

$$
R_{2}=\min \left[1.0,100\left(1-\alpha_{v}\right)\right]
$$




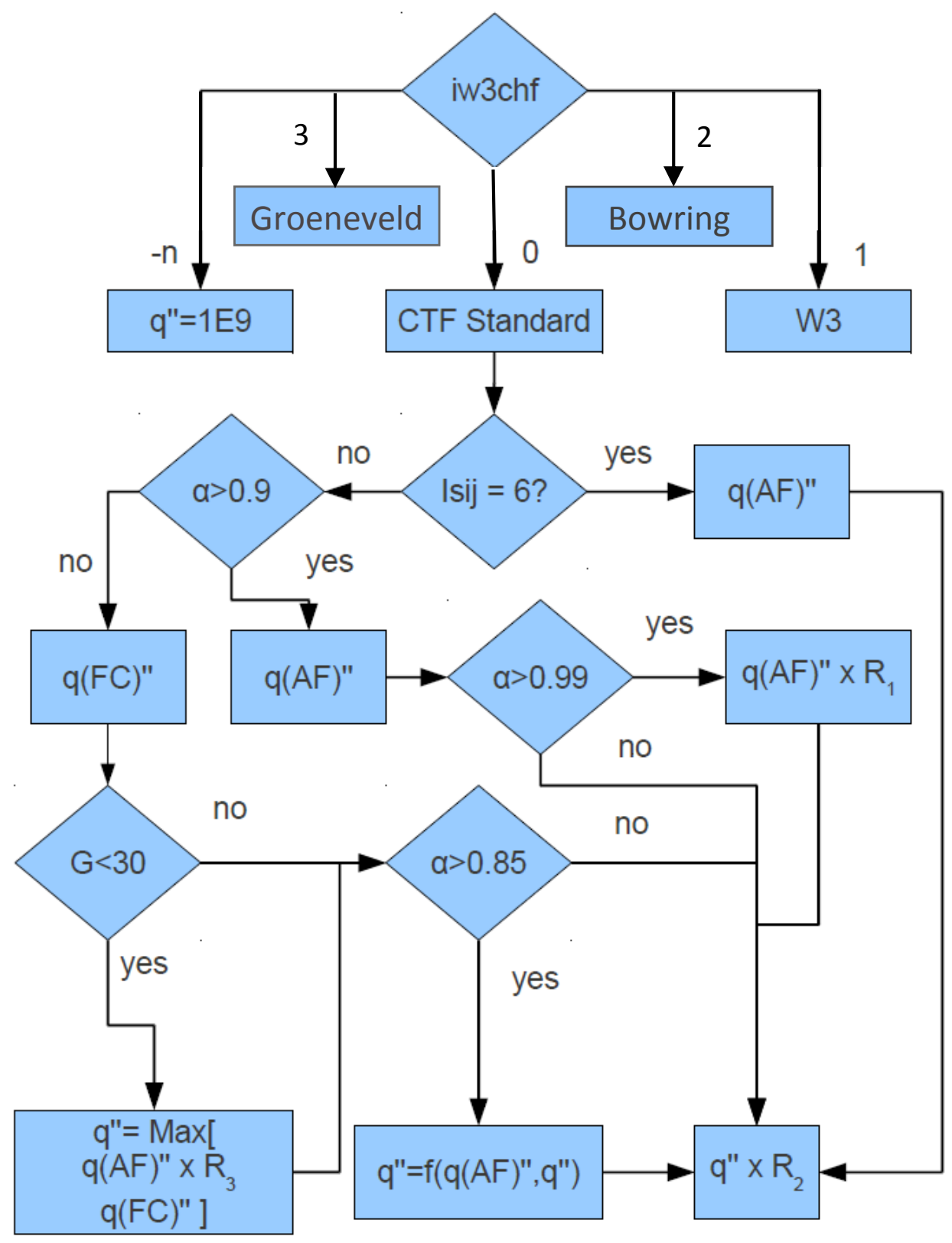

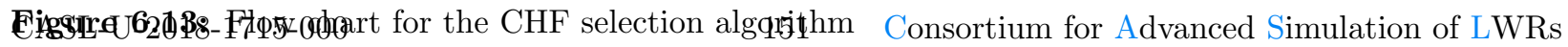


With the overview of the CHF selection algorithm complete, we point our attention to defining the actual CHF models employed.

Pool Boiling The pool boiling critical heat flux is given by modification of the Zuber et al. equation [72], as recommended by Bjornard and Griffith [73]. The Zuber equation is shown in Equation 6.63.

$$
q_{c h f}^{\prime \prime}=0.9\left(1-\alpha_{v}\right) \frac{\pi}{24} H_{f g} \rho_{g}^{0.5}\left[g_{c} g \sigma\left(\rho_{f}-\rho_{g}\right)\right]^{0.25}
$$

The constant recommended by Zuber, $0.9 \pi / 24$, is not used in CTF. The data presented by Zuber showed a range in the value of the leading constant going from 0.10 to 0.24 (compared to the recommended value of 0.1178 ). For CTF, the average of this range was taken (0.17) and it was multiplied by 0.9 , which is a multiplication factor for vertical rod geometry recommended by Lienhard and Dhir [74]. This leads to a leading coefficient of 0.15 .

$$
q_{\mathrm{chf}}^{\prime \prime}=0.15\left(1-\alpha_{v}\right) H_{\mathrm{fg}} \rho_{g}^{0.5}\left[g_{c} g \sigma\left(\rho_{f}-\rho_{g}\right)\right]^{0.25} R_{3}
$$

The ramping factor, $R_{3}$, ranges between 0.2 and 1.0, with the magnitude increasing as liquid void decreases. It is defined as:

$$
R_{3}=\max \left[0.2,\left(1-\alpha_{v}\right)\right]
$$

Equation 6.64 is compared against the critical heat flux calculated with the forced convective boiling model, which is discussed in the following section. The maximum of Equation 6.64 and 6.68 is used for the pool boiling critical heat flux.

Forced Convection Boiling The forced convection critical heat flux is given by the Biasi correlation. CTF uses the modified form of the Biasi correlation given by Sardh and Becker [75], which differs slightly from the original formulation given by Biasi [76]. The correlation consists of equations for low-quality and high-quality flow.

$$
\begin{aligned}
& q_{\mathrm{b} 1}^{\prime \prime}=8,761,800 \cdot G^{-1 / 6}\left[1.468 F(P) G^{-1 / 6}-x\right] D_{h}^{-n} \\
& q_{\mathrm{b} 2}^{\prime \prime}=47,701,900 \cdot H(P)(1-x) D_{h}^{-n} G^{-0.6}
\end{aligned}
$$

Where,

$q^{\prime \prime}=$ critical heat flux $\left(\mathrm{BTU} / \mathrm{hr} \cdot \mathrm{ft}^{2}\right)$

$G=\operatorname{mass}$ flux $\left(\mathrm{g} / \mathrm{cm}^{2} \cdot \mathrm{sec}\right)$ 
$n= \begin{cases}0.6 & \text { if } D_{h}<1.0 \mathrm{~cm} \\ 0.4 & \text { if } D_{h}>1.0 \mathrm{~cm}\end{cases}$

The functions, $F(P)$ and $H(P)$, are functions of pressure in bars and are calculated as follows:

$$
\begin{aligned}
& F(P)=0.7249+0.099 P \exp (-0.032 P) \\
& H(P)=-1.159+0.149 P \exp (-0.019 P)+8.99 P\left(10+P^{2}\right)^{-1}
\end{aligned}
$$

The critical heat flux is then calculated as the maximum of $q_{\mathrm{b} 1}^{\prime \prime}, q_{\mathrm{b} 2}^{\prime \prime}$, or a fixed value of $90,000 \mathrm{BTU} / \mathrm{hr} \cdot \mathrm{ft}^{2}$.

$$
q_{\mathrm{chf}, \mathrm{fc}}^{\prime \prime}=\max \left\{\begin{array}{c}
q_{\mathrm{b} 1}^{\prime \prime} \\
q_{\mathrm{b} 2}^{\prime \prime} \\
90,000^{\mathrm{BTU} / \mathrm{hr}_{\mathrm{ft}}{ }^{2}}
\end{array}\right.
$$

Annular Film Dryout In this region, DNB occurs due to the film drying out, which is a complex function of several variables. Though critical heat flux in the annular film dryout region is not limited by correlation, the critical heat flux is calculated using the Zuber equation, which was also used for pool boiling. The form used for the annular film dryout region is shown in Equation 6.69.

$$
q_{\mathrm{chf}}^{\prime \prime}=0.15\left(1-\alpha_{v}\right) H_{f g} \rho_{g}^{0.5}\left[g_{c} g \sigma\left(\rho_{f}-\rho_{g}\right)\right]^{0.25}
$$

If the mesh cell is not in a hot wall flow regime and the void fraction is greater than 0.99, Equation 6.69 will be multiplied by a ramping factor to reduce CHF as liquid disappears.

$$
R_{1}=\max \left[0.2,200\left(\alpha_{l}-0.005\right)\right]
$$

\subsubsection{W-3 Correlation}

The W-3 correlation, developed by Tong ([77],[78]) is the most widely used correlation for evaluating DNB in PWRs [79]. The critical heat flux is determined by use of the following equation:

$$
\begin{aligned}
& \frac{q_{\mathrm{w} 3, \mathrm{D}_{\text {heated }}, u}^{\prime \prime}}{10^{6}}=\{(2.022-0.0004302 p)+(0.1722-0.0000984 p) \\
&\left.\exp \left[(18.177-0.004129 p) x_{e}\right]\right\}\left[\left(0.1484-1.596 x_{e}+0.1729 x_{e}\left|x_{e}\right|\right) \frac{G}{10^{6}}+\right. \\
&1.037]\left(1.157-0.869 x_{e}\right)\left[0.2664+0.8357 \exp \left(-3.151 D_{\text {heated }}\right)\right] \\
& {\left[0.8258+0.000794\left(h_{f}-h_{\text {in }}\right)\right] }
\end{aligned}
$$


Where,

$$
\begin{aligned}
& q_{\mathrm{w} 3, \mathrm{D}_{\text {heated }}, u}^{\prime \prime}=\text { critical heat flux based on heated diameter }\left[\mathrm{BTU} /{\left.\mathrm{hr} \cdot \mathrm{ft}^{2}\right]}^{2}\right. \\
& p=\text { pressure [psia] } \\
& x_{e}=\text { local steam thermodynamic quality } \\
& D_{\text {heated }}=\text { heated hydraulic diameter }[\text { in }]= \\
& h_{\text {in }}=\text { inlet enthalpy [BTU/lbm] } \\
& G=\operatorname{mass} \text { flux }\left[\mathrm{lbm} / \mathrm{hr} \cdot \mathrm{ft}^{2}\right]
\end{aligned}
$$

The W-3 correlation is applicable to the following range of operating conditions:

$$
\begin{aligned}
p & =800 \text { to } 2300 \mathrm{psia} \\
\frac{G}{10^{6}} & =1.0 \text { to } 5.0 \mathrm{lbm} /{\mathrm{hr} \cdot \mathrm{ft}^{2}}^{2} \\
D_{\text {heated }} & =0.2 \text { to } 0.7 \mathrm{in} \\
x_{e} & =-0.15 \text { to } 0.15 \\
L & =10 \text { to } 144 \mathrm{in}
\end{aligned}
$$

Note that the original form of the W-3 correlation developed by Tong used the hydraulic diameter in place of the heated hydraulic diameter in Equation 6.71. However, Tong developed a cold wall factor to account for the effect of unheated surfaces. Consider two separate heated channels with the same hydraulic conditions (including the same wetted perimeter) and the same total heat added per unit length; if one of these channels is connected only to heated surfaces but the other channel is connected to a combination of heated and unheated surfaces, the latter channel is expected to have a lower CHF value because there is less total liquid flow adjacent to the heated surfaces and therefore less ability to remove heat from the wall.

The cold wall factor provided by Tong is given by:

$$
\begin{aligned}
\frac{q_{\mathrm{w} 3, \text { cold wall, }}^{\prime \prime}}{q_{\mathrm{w} 3, \mathrm{D}_{\text {heated }}, \mathrm{u}}^{\prime \prime}}= & 1.0-\mathrm{Ru}\left[13.76-1.372 e^{1.78 x_{e}}\right. \\
& -4.732\left(\frac{G}{10^{6}}\right)^{-0.0535}-0.0619\left(\frac{p}{10^{3}}\right)^{0.14} \\
& \left.-8.509\left(D_{\text {heated }}\right)^{0.107}\right]
\end{aligned}
$$

where:

$$
R u=1-\frac{D_{h}}{D_{\text {heated }}}
$$

In CTF, the cold wall factor calculation is always enabled when using the W-3 correlation, and this factor is used in conjunction with the form of Equation 6.71 using the heated hydraulic diameter, as prescribed by Tong. 
Equation 6.72 gives the CHF for uniform axial heat flux profiles; however, the W-3 model also includes a factor for handling non-uniform axial heat flux profiles, known as the Tong F-factor. The factor is applied as shown below in order to provide a CHF for non-uniformly heated rods.

$$
q_{\mathrm{chf}, \text { cold wall,nu }}^{\prime \prime}=\frac{q_{\mathrm{w} 3, \text { cold wall,u }}^{\prime \prime}}{F}
$$

The uniform CHF, calculated by Equation 6.71, is represented by $q_{\mathrm{w} 3, \mathrm{u}}^{\prime \prime}$ and the CHF for non-uniformly heated rods is represented by $q_{\mathrm{w} 3, \mathrm{nu}}^{\prime \prime}$. The Tong F-factor is meant to capture the memory effect of the flow in CHF conditions. The boundary layer energy and bubble content affects the occurrence of CHF in both PWRs and boiling water reactor (BWR)s. In the PWR, the boundary layer carries bubbles that result in a local peak of void near the wall, with a thin liquid film coating the wall. As energy and heat flux increases, the film may evaporate, which triggers CHF. In the BWR, the flow regime is typically annular, with a vapor core in the channel center and a liquid film coating the wall. Again, the film may dry out as heat flux increases and liquid subcooling decreases, resulting in CHF that depends on the local quality, mass flux, and pressure. In addition to the local effect, for BWRs the power shape and heated length affect CHF incipience as well due to the hydrodynamic interaction between the vapor core, liquid film, and wall boiling along the length of the channel. The CHF occurrence, therefore, is dependent on the upstream conditions that dictate local energy and void content of the flow as well as phase interactions at high quality.

Tong used a theoretical basis to develop the F-Factor by analyzing a control volume over the boundary layer. His assessment takes upstream heating into account, resulting in an integration from the axial location where boiling starts to the current location being analyzed. This relationship is shown in Equation 6.74.

$$
F=\frac{C \int_{0}^{l} q^{\prime \prime}\left(z^{\prime}\right) \exp \left(\left[-C *\left(l-z^{\prime}\right)\right] d z^{\prime}\right.}{q^{\prime \prime} * l[1-\exp (-C * l)]}
$$

Note that the lower bound of the definite integration, 0, is referring to the onset of nucleate boiling (ONB) location. The $l$ refers to the current axial location where CHF is being calculated. The Tong factor calculation has been implemented into CTF using a semi-analytical approach. This is done by solving the integral analytically for each rod level, assuming the heat flux is constant over that axial level. This is consistent with the control volume approach of CTF; all rod properties are constant for a discrete axial level. The analytical solutions are summed for each level from 0 to $l$ to form an approximation of the total integral. In equation form, this looks as follows:

$$
\frac{\sum_{j=J_{\mathrm{ONB}}}^{J_{l}} q^{\prime \prime}(j)\left[\exp \left(-C\left(z\left(J_{l}\right)-z(j+1 / 2)\right)\right)-\exp \left(-C\left(z\left(J_{l}\right)-z(j-1 / 2)\right)\right)\right]}{q^{\prime \prime}\left(J_{l}\right)\left(1-\exp \left(-C z\left(J_{l}\right)\right)\right)}
$$

The $j$ term is a counter over the discrete axial levels in the integration. $J_{\mathrm{ONB}}$ is the axial level where ONB has occured and $J_{l}$ is the current axial level being analyzed in the code. The heat flux, $q^{\prime \prime}(j)$, is constant for each axial level, $j$. The term in square brackets is the analytical solution of the definite integral for the given level, $j$. We substitute the axial location at the top, $z(j+1 / 2)$, and bottom, $z(j-1 / 2)$, of the axial level, $j$. Other terms include the axial location at the center of the current cell, $z\left(J_{l}\right)$, and the heat flux at the current axial level, $q^{\prime \prime}\left(J_{l}\right)$. 
The $C$ term is a factor that accounts for flow quality and mass flux (defined in Equation 6.76).

$$
C=0.15 \frac{\left(1.0-x_{\mathrm{DNB}}\right)^{4} .31}{\left(G / 10^{6}\right)^{0.478}}
$$

$G$ is the local mass flux near the rod surface, which is in units of $1 \mathrm{bm} / \mathrm{hr}^{-\mathrm{ft}^{2}}$ and $x$ is the equilibrium quality adjacent the surface. The $C$ factor has units of in ${ }^{-1}$, which is converted to $\mathrm{ft}^{-1}$ before being used in Equation 6.74. Note that, in a subcooled flow, $C$ will be large and $F$ will, in turn, be close to 1.0, which means the local CHF will be determined by the local CHF predicted by the W-3 correlation. In flows with a high quality content, $C$ will be small, $F$ large, and local CHF will be determined largely by upstream flow effects.

Note that the Tong F factor is calculated for each rod surface and level. This is different from the W-3 calculated CHF value given by Equation 6.71, which is calculated for each fluid cell in the mesh. The Tong $\mathrm{F}$ factor is then applied to the adjacent fluid-cell CHF, so that a CHF value is obtained for each rod surface and level. Also note that the Tong F-factor is not calculated for surfaces where boiling is not occurring; the F-factor is simply assigned a value of 1.0 in this case. Therefore, rods with no boiling along the axial length will be assigned an F-factor of 1.0 everywhere and will simply retain the uniform CHF value obtained from Equation 6.71. The Tong F-factor is calculated automatically when the W-3 CHF correlation is selected; it is skipped when the $\mathrm{W}-3$ correlation is not selected.

\subsubsection{Bowring Correlation}

Developed by R.W. Bowring [80], the Bowring CHF correlation was developed for circular ducts under a wide range of pressures and mass-fluxes. His correlation is based on experimental data from uniformly heated tubes. With a known subcooling, pressure, mass flux and tube length, Bowring would increase the heating rate to each tube until DNB is achieved at the exit. The Bowring correlation is then applicable to the following range of operating conditions:

$$
\begin{aligned}
p & =34 \text { to } 2,750 \mathrm{psi} \\
G & =0.1 \text { to } 13.7 \mathrm{Mlb} /{\mathrm{hr} \cdot \mathrm{ft}^{2}}^{2} \\
D_{h} & =0.08 \text { to } 1.76 \mathrm{in} \\
L & =6.0 \text { to } 144.0 \mathrm{in}
\end{aligned}
$$

In his original paper, R.W. Bowring presents the equation for the CHF in the form:

$$
q_{\mathrm{chf}}^{\prime \prime}=\frac{A-B \Delta h_{\text {sub }, i n}}{C+L}
$$

Where $\Delta h_{s u b, i n}$ is the subcooling at the inlet, and $L$ is the length at which CHF occurs. The remaining portions of the correlation can be described as follows using traditional British units: 


$$
\begin{gathered}
A=\frac{2.317\left(h_{f g} B\right) F_{1}}{1+3.092 F_{2} D_{h}^{1 / 2} G} \\
B=\frac{D G}{4} \\
C=\frac{104.4 F_{3} D G}{1+0.347 F_{4} G^{n}} \\
n=2.0-0.5 p_{R} \\
p_{R}=0.001 p
\end{gathered}
$$

And the pressure dependent constants are:

For $p_{R}<1$ psi,

$$
\begin{aligned}
& F_{1}=\frac{p_{R}^{18.942} \exp \left[20.89\left(1-p_{R}\right)\right]+0.917}{1.917} \\
& F_{2}=\frac{1.309 F_{1}}{p_{R}^{1.316} \exp \left[2.444\left(1-p_{R}\right)\right]+0.309} \\
& F_{3}=\frac{p_{R}^{17.023} \exp \left[16.658\left(1-p_{R}\right)\right]+0.667}{1.667} \\
& F_{4}=F_{3} p_{R}^{1.649}
\end{aligned}
$$

For $p_{R}>1$ psi,

$$
\begin{aligned}
& F_{1}=p_{R}^{-0.368} \exp \left[0.648\left(1-p_{R}\right)\right] \\
& F_{2}=\frac{F_{1}}{p_{R}^{-0.448} \exp \left[0.245\left(1-p_{R}\right)\right]} \\
& F_{3}=p_{R}^{0.219} \\
& F_{4}=F_{3} p_{R}^{1.649}
\end{aligned}
$$

The form given in Equation 6.77 is restrictive in that it requires knowledge of where the CHF occurs. A modified form can be obtained though by first algebraically manipulating Equation 6.77: 


$$
q_{\mathrm{chf}}^{\prime \prime}=\frac{A-B\left(q_{\mathrm{chf}}^{\prime \prime} \frac{L}{B}-\Delta h_{\text {sub }, \text { in }}\right)}{C}
$$

Because Bowring's experiment was performed for uniformly heated tubes, an expression for the equilibrium quality can be developed from an energy balance:

$$
x_{e}(z)=q_{o}^{\prime \prime} \frac{z}{B h_{f g}}-\frac{\Delta h_{s u b, i n}}{h_{f g}}
$$

Where $q_{o}^{\prime \prime}$ is the local heat flux at the axial location $z$. The critical equilibrium quality corresponding to the CHF can then be developed as follows:

$$
x_{e, c r i t} h_{f g}=q_{\mathrm{chf}}^{\prime \prime} \frac{L}{B}-\Delta h_{s u b, i n}
$$

Substituting Equation 6.87 into Equation 6.85 and treating the critical equilibrium quality as the local equilibrium quality yields the final result for the modified Bowring CHF:

$$
q_{\mathrm{chf}}^{\prime \prime}=\frac{A-B h_{f g} x_{e}}{C}
$$

The above expression is independent of the CHF location, and yields the critical heat flux corresponding to any local equilibrium quality. This form is encoded into CTF for the Bowring correlation.

\subsubsection{Groeneveld Look-up Tables}

Developed by D. C. Groeneveld et al [81] from available experimental data in $8 \mathrm{~mm}$ tubes, the Groeneveld CHF look-up tables are simply a collection of tabulated values for the CHF as a function of equilibrium quality, pressure and mass flux. The resulting Groeneveld CHF can be expressed as follows:

$$
q_{\mathrm{chf}}^{\prime \prime}=q_{\mathrm{LUT}}^{\prime \prime} \prod_{i=1}^{8} K_{i}
$$

Where $q_{\mathrm{LUT}}^{\prime \prime}$ is the interpolated CHF from the look-up tables. The Groeneveld look-up tables are applicable in the following range of operating conditions:

$$
\begin{aligned}
p & =0.1 \text { to } 21 \mathrm{MPa} \\
G & =0.0 \text { to } 8,000 \mathrm{~kg} / \mathrm{s} \cdot \mathrm{m}^{2} \\
x_{e} & =-0.5 \text { to } 0.9 \\
D_{h} & =0.003 \text { to } 0.025 \mathrm{~m}
\end{aligned}
$$


The look-up tables are stored in CTF as a $15 \times 21 \times 23$ array in pressure, mass-flux and equilibrium quality. The interpolation scheme begins by reducing the large array to a $2 \times 2 \times 2$ array that bounds the current state point. This resulting $2 \times 2 \times 2$ array is then linearly interpolated in pressure, then in mass-flux, and finally in equilibrium quality, resulting in a final interpolated value for $q_{\text {LUT }}^{\prime \prime}$. This can be seen in detail in the CTF Validation and Verification Manual [82].

In the case that CTF calculates a parameter that is out-of-bounds in the Groeneveld look-up tables, the value will be reset to the boundary. For instance, if the value $x_{e}=-0.7$ was passed to the Groeneveld function in CTF, it would be reset to $x_{e}=-0.5$ (the lowest boundary) and the calculation would proceed. This makes the encoded Groeneveld function in CTF particularly difficult to break.

The Groeneveld look-up table values are accompanied by eight available form or "K" factors [83] [84] which adjust the interpolated CHF value for various bundle or subchannel effects. The first available K-factor scales the look-up table CHF as determined from $8 \mathrm{~mm}$ tubes to a generic tube (or hydraulic) diameter:

$$
K_{1}=\left\{\begin{array}{lll}
\left(\frac{0.008}{D_{h}}\right)^{1 / 2} & \text { for } & 0.003<D_{h}<0.025 \mathrm{~m} \\
0.57 & \text { for } & D_{h} \geq 0.025 \mathrm{~m}
\end{array}\right.
$$

The second K-factor scales bundle effects for subchannel considerations. This K-factor would be applied to a bundle averaged solution in order to reconstruct subchannel effects:

$$
\begin{aligned}
K_{2} & =\min \left[1,\left(\frac{1}{2}+\frac{2 \delta}{D_{\text {rod }}}\right) \exp \left(-\frac{x_{e}^{1 / 3}}{2}\right)\right] \\
\delta & =\text { Pitch }-D_{\text {rod }}
\end{aligned}
$$

The next applied K-factor describes the perturbation in the CHF from enhanced mixing due to spacer grids:

$$
\begin{aligned}
K_{3} & =1+A \exp -\frac{0.1 L_{s p}}{D_{h}} \\
A & =1.5\left(k_{\text {loss }}\right)^{0.5}\left(\frac{G}{1000}\right)^{0.2}
\end{aligned}
$$

Where $L_{s p}$ is the distance upstream from the spacer grid, and $k_{\text {loss }}$ is the spacer grid loss coefficient. The so called "heated length" K-factor is described as follows, for $L / D_{h}>5$ :

$$
\begin{aligned}
K_{4} & =\exp \left[\left(\frac{D_{h}}{L}\right) \exp \left(2 \alpha_{H E M}\right)\right] \\
\alpha_{H E M} & =\frac{x_{e} \rho_{f}}{x_{e} \rho_{f}+\left(1-x_{e}\right) \rho_{g}}
\end{aligned}
$$


Where $\alpha_{H E M}$ is the void fraction as defined in the homogeneous equilibrium model. The value is initialized as zero in CTF, and only calculated for non-negative $x_{e}$. Finally, the axial heat flux distribution K-factor scales the CHF for non-uniform heating:

$$
K_{5}=\left\{\begin{array}{lll}
1.0 & \text { for } \quad x_{e} \leq 0 \\
q^{\prime \prime}(z) / q_{B L A}^{\prime \prime} & \text { for } \quad x_{e}>0
\end{array}\right.
$$

Where $q_{B L A}^{\prime \prime}$ is the boiling length averaged heat flux. This final $\mathrm{K}$-factor is similar in purpose to the W-3 correlation's Tong factor. Unlike factors $K_{1}$ through $K_{4}$, which are fluid cell properties, the $K_{5}$ factor is a rod-surface dependent property.

The next K-factor accompanying the Groeneveld look-up table scales the effect of the local radial power for radially non-uniform bundle power:

$$
K_{6}=\left\{\begin{array}{lll}
1.0 & \text { for } & x_{e} \leq 0 \\
q^{\prime \prime}(z)_{\text {avg }} / q^{\prime \prime}(z)_{\max } & \text { for } & x_{e}>0
\end{array}\right.
$$

Similar to $K_{2}$, this $\mathrm{K}$-factor would be used to reconstruct local pin power effects from a bundle averaged calculation. The seventh K-factor shows the effect on CHF from varying flow orientation, specifically due to cross-flow:

$$
\begin{aligned}
& K_{7}=1-\exp \left[-\left(\frac{T_{1}}{3}\right)^{0.5}\right] \\
& T_{1}=\left(\frac{1-x_{e}}{1-\alpha}\right)^{2} \frac{f_{L} G^{2}}{g D_{h} \rho_{f}\left(\rho_{f}-\rho_{g}\right) \alpha^{0.5}}
\end{aligned}
$$

Where $f_{L}$ is the friction factor of the channel, and $\alpha$ is the void fraction. The final $\mathrm{K}$-factor determines the effect of low vertical flow or reflood on the CHF:

$$
\begin{aligned}
& \left\{\begin{array}{ccc}
K_{8}=1.0 & \text { for } & G<-400 \mathrm{~kg} / \mathrm{s} \cdot \mathrm{m}^{2} \\
\text { Linear interpolation between } q_{L U T}^{\prime \prime} & & \\
\text { from }|G| \text { and value predicted from } & & \\
q_{L U T}^{\prime \prime}=q_{L U T, G=0, x_{e}=0}^{\prime \prime}\left(1-\alpha_{H E M}\right) C_{1} & \text { for } & -400 \leq G<0 \mathrm{~kg} / \mathrm{s} \cdot \mathrm{m}^{2}
\end{array}\right. \\
& C_{1}=\left\{\begin{array}{cccc}
1.0 & \text { for } & \alpha_{H E M}<0.8 \\
\frac{0.8+0.2 \rho_{f} / \rho_{g}}{\alpha_{H E M}+\left(1-\alpha_{H E M}\right) \rho_{f} / \rho_{g}} & \text { for } & \alpha_{H E M}>0.8
\end{array}\right.
\end{aligned}
$$

Where $G=0, x_{e}=0$ refers to a pool boiling situation.

Currently, only $K_{1}, K_{4}$ and $K_{5}$ are encoded into CTF. $K_{2}$ is not currently considered as individual subchannel geometry is already being accounted for [84]. Likewise, $K_{6}$ and $K_{7}$ are not considered because cross-flow 
and non-uniform radial heating is already being accounted for. The spacer grid k-factor, $K_{3}$, is not being used because the Yao, Hochreiter, and Leech correlation [85] is already accounting for enhanced mixing and heat transfer upstream of spacer grids. The $K_{8}$ factor is also not considered at this time.

\subsubsection{Calculation of $T_{\text {chf }}$}

The CHF temperature is calculated iteratively using the previously-calculated CHF heat flux. The algorithm is summarized visually in the flowchart in Figure 6.14. The first step is setting minimum and maximum boundaries for $T_{\text {chf }}$. These minimum and maximum boundaries are $0.01^{\circ} \mathrm{F}$ over the liquid saturation temperature for the minimum boundary and the maximum of $75^{\circ} \mathrm{F}$ over the saturation temperature or the critical temperature of water $\left(705.3^{\circ} \mathrm{F}\right)$ for the maximum boundary.

The code then checks to see if the calculated CHF exceeds either of the boundaries. If yes, the CHF and $T_{\text {chf }}$ values are set to the boundary that is exceeded. If not, the iterative process to find the actual $T_{\text {chf }}$ begins. The process is to take the midpoint between $T_{\min }$ and $T_{\max }$, use that to calculate heat flux at that temperature using the nucleate boiling correlation (i.e., Chen[86] or Thom[87]), and then compare that heat flux to the critical heat flux that was previously calculated. If $q_{\mathrm{chf}}^{\prime \prime}$ is less than $q_{\mathrm{nb}}^{\prime \prime}$ (the nucleate boiling heat flux), the new minimum temperature becomes the current $T_{\text {chf }}$ temperature. If $q_{\text {chf }}^{\prime \prime}$ is larger than $q_{\mathrm{nb}}^{\prime \prime}$, the new maximum temperature becomes $T_{\text {chf }}$.

This effectively cuts the range of temperatures in half and the next iteration commences using the new midpoint temperature. This process continues until $q_{\mathrm{nb}}^{\prime \prime}$ matches $q_{\mathrm{chf}}^{\prime \prime}$ to within a relative error of 0.01 . The temperature that led to the correct $q_{\mathrm{nb}}^{\prime \prime}$ value is returned as the temperature at CHF.

An example of this process is shown in Figure 6.15.

\subsection{Heat Transfer Models}

\subsubsection{Introduction}

Aside from wall drag and form losses, solid components in the flow field may also contribute in another way to the formulation of the flow field conservation equations - energy transfer from heated objects. At this point in time, it is unimportant whether energy is coming from electrically heated rods or actual nuclear fuel rods. Instead, we are only interested in the heat transfer coefficient $(h)$ of the liquid-solid interface and the temperatures of the solid surface $\left(T_{w}\right)$ and the liquid in contact with the solid surface $\left(T_{F}\right)$. As seen in Equation 6.98, this information allows for the calculation of the heat input into the liquid or, numerically speaking, into the continuity mesh cell neighboring the heated solid surface.

$$
q^{\prime \prime}=h\left(T_{w}-T_{F}\right)
$$

The solid surface temperature, $T_{w}$, will be available from the conduction equation solution. The fluid temperature, $T_{F}$, will be available from the conservation equation solution (discussed in Chapter 2). The calculation of the heat transfer coefficient is discussed in this section. Sections 6.6.3 through 6.6.9 discuss 


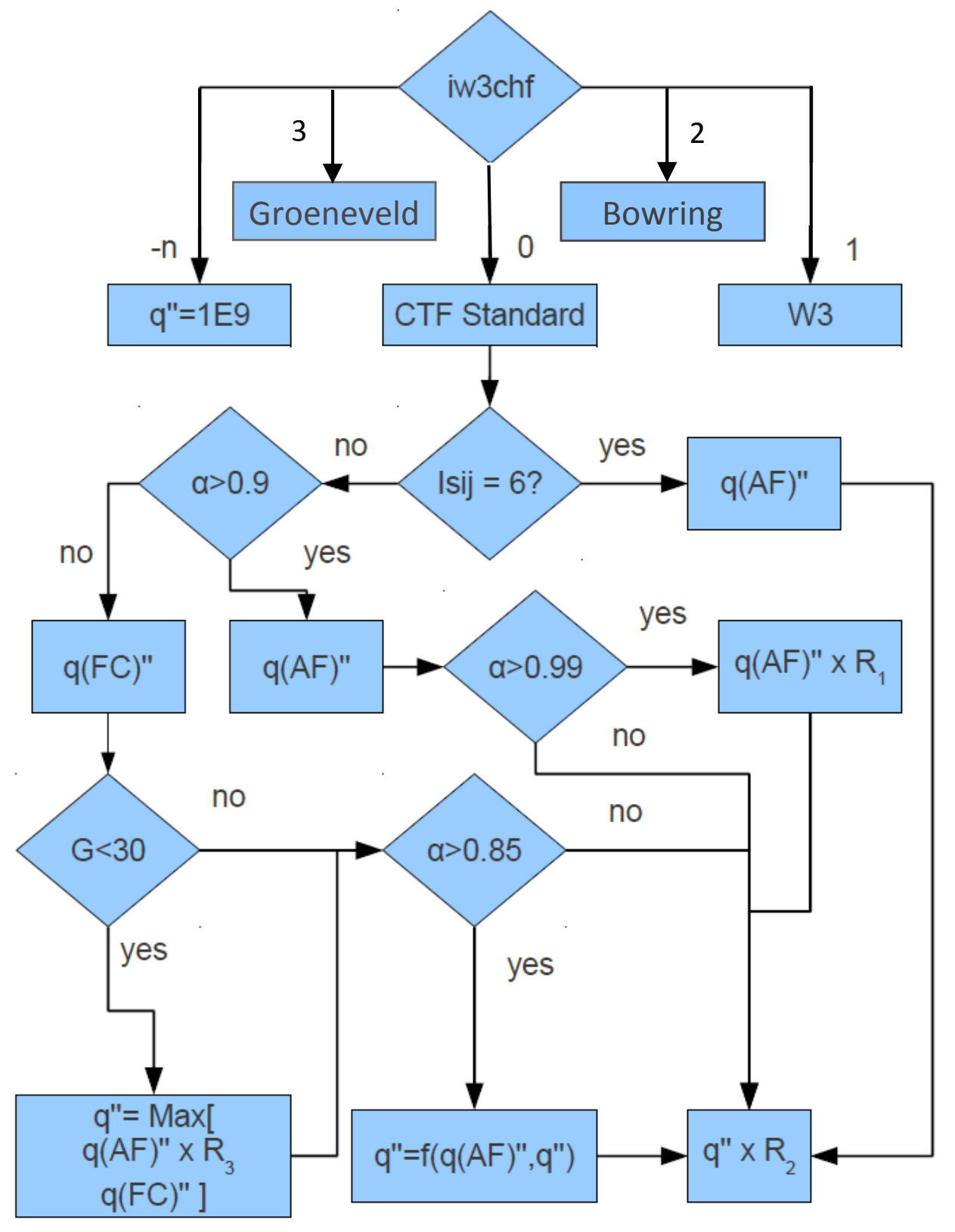




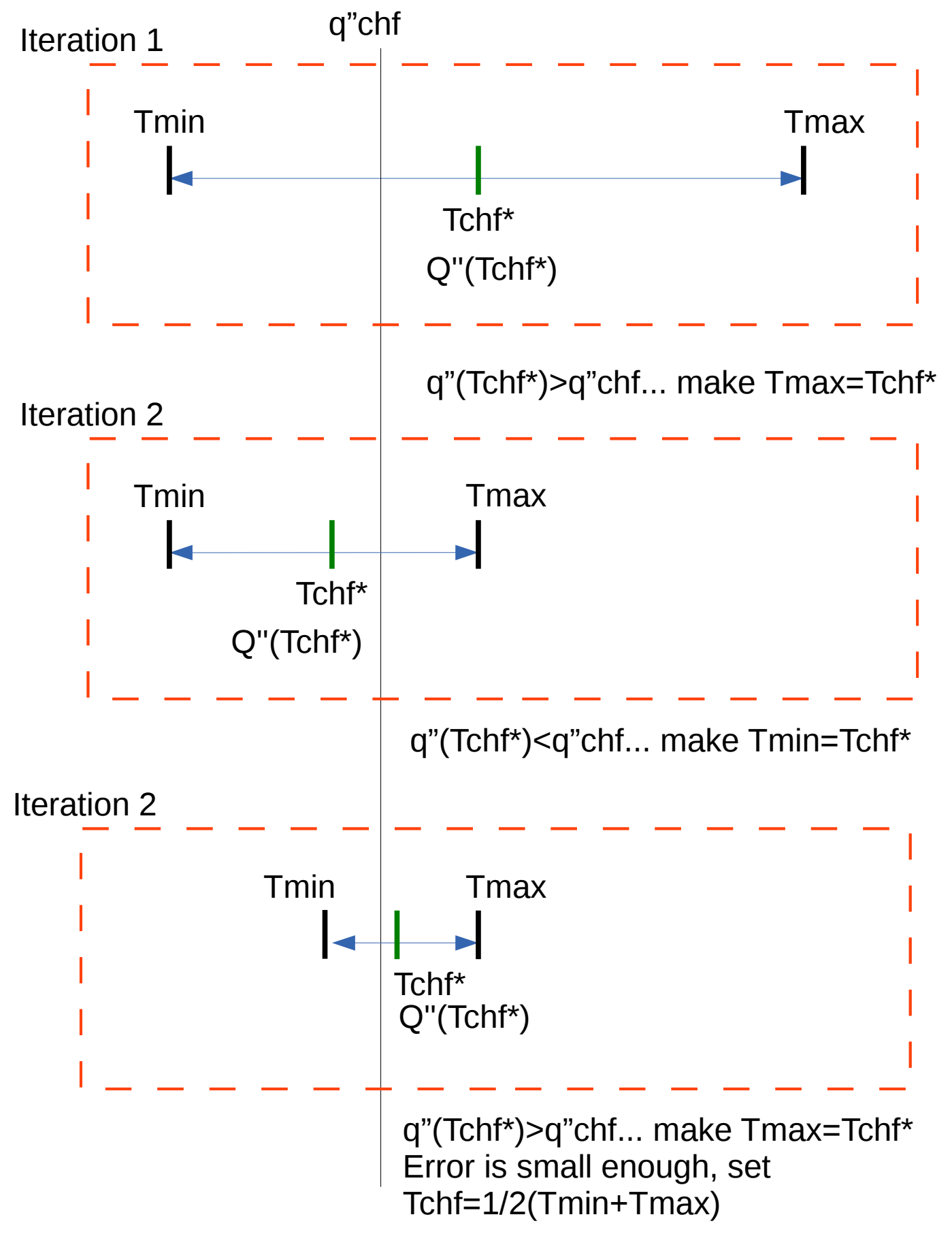

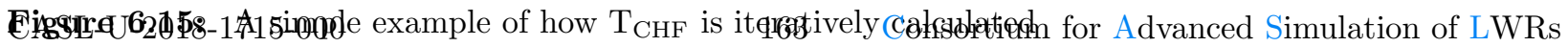


the modeling of convective heat transfer for all heat transfer regimes modeled by CTF. Section 6.6 .10 covers radiative heat transfer and Section 6.6.11 covers grid heat transfer and the grid re-wet model.

The heat transfer coefficient is highly dependent on the behavior of the flow and is calculated from correlation. Different models are used for each heat transfer "region". Heat transfer regions are depicted in Figure 6.12, which presents the boiling curve. Moving from left to right in the figure, heat flux begins to increase dramatically when a certain temperature difference between the heated surface and adjacent fluid is reached. This corresponds to the beginning of boiling and the increased heat removal that accompanies the phenomenon. Heat removal continues to rise with increased wall temperature until the vapor phase begins to blanket the heated surface and prevent further increase in heat removal - an occurrence known as DNB or CHF.

From this point, increased temperature causes growth of the vapor blanket and further reduction in the heat flux. The heat flux bottoms out at the minimum film boiling point $\left(T_{\min }\right)$ where the heated surface is completely blanketed in vapor and the liquid phase is suspended above that blanket. From this point, further increasing the surface temperature causes increased radiative heat transfer through the vapor film, which resumes the increase in heat flux with respect to surface temperature.

CTF recognizes the following heat transfer regimes:

1. Single-phase liquid convection (SPL)

2. Single-phase vapor convection (SPV)

3. Subcooled nucleate boiling (SCB)

4. Saturated nucleate boiling (NB)

5. Transition boiling (TRAN)

6. Inverted annular film boiling (IAFB)

7. Dispersed droplet film boiling (IADF)

8. Dispersed droplet deposition heat transfer (DFFB)

$\mathrm{CTF}$ selects the appropriate heat transfer regime after evaluating several criteria. If the heated surface temperature is less than $0.1^{\circ} \mathrm{F}$, a pre-CHF heat transfer regime will be selected (i.e. single-phase liquid convection, subcooled nucleate boiling, or saturated nucleate boiling). Note that the single-phase vapor convection regime will be selected if the void fraction is above 0.999 , regardless of the heated surface temperature (see Section 6.6.3).

On the other hand, if the heated surface temperature is greater than or equal to $0.1^{\circ} \mathrm{F}$ above the critical heat flux temperature, then one of the post-CHF heat transfer regimes will be selected (i.e. transition boiling, inverted annular film boiling, dispersed droplet film boiling, or dispersed droplet deposition heat transfer). A further distinction is made by selecting the transition boiling regime if the heated wall temperature is less than the minimum film boiling temperature and one of the other post-CHF regimes if the heated wall temperature is larger than the minimum film boiling temperature. An overview of the heat transfer region selection algorithm in CTF is depicted in Figure 6.16 


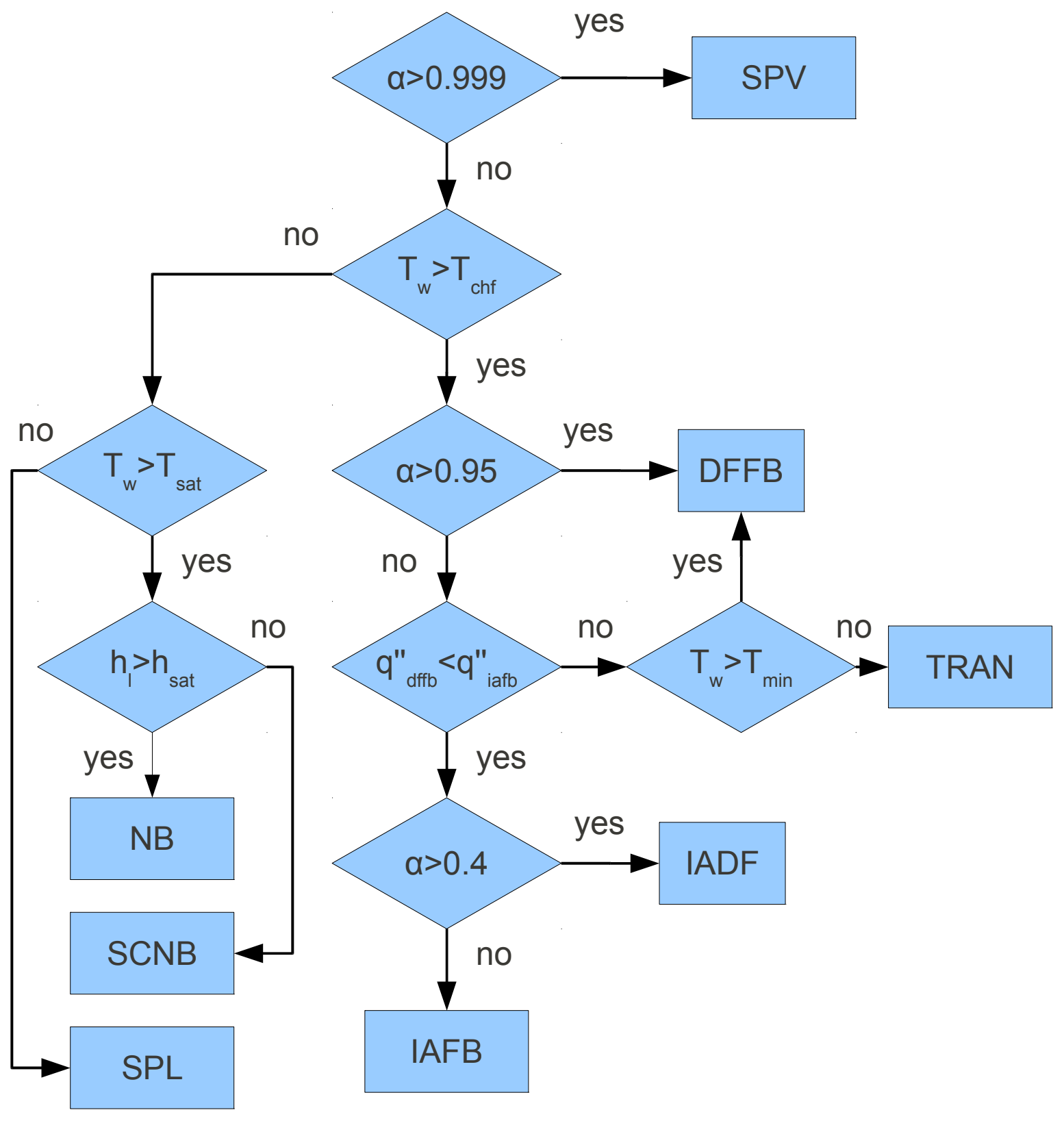

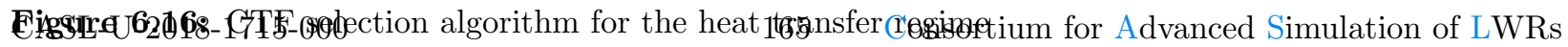


Prior to determining the heat transfer regime in the aforementioned manner, it is necessary to first define the necessary criteria: the CHF temperature, $T_{\text {chf }}$, and the minimum film boiling temperature $T_{\min }$. The definitions of these criteria are now discussed.

\subsubsection{Minimum Film Boiling Temperature}

The minimum film boiling temperature is the temperature at which a stable film forms on the heated surface. It separates the transition boiling region, where liquid intermittently contacts the heated surface, and the film boiling region, where the surface is too hot to ever allow liquid to contact. For unheated conductors, this temperature is set to a constant value of $900^{\circ} \mathrm{F}$. For heated structures, the minimum film boiling temperature is set to a minimum value of $900^{\circ} \mathrm{F}$ for void fractions less than 0.8 and to a minimum value of $700^{\circ} \mathrm{F}$ for void fractions equal to or greater than 0.8. The minimum film boiling temperature may be much higher, though, and is evaluated using two different methods.

First, $T_{\min }$ is calculated assuming it equals the wall temperature that results in an instantaneous contact temperature equal to the homogeneous nucleation temperature. The homogeneous nucleation temperature for water is a function of pressure.

$$
T_{\text {hn }}=705.44-4.722 \times 10^{-2}\left(P_{\text {crit }}-P\right)+2.3907 \times 10^{-5}\left(P_{\text {crit }}-P\right)^{2}-5.8193 \times 10^{-9}\left(P_{\text {crit }}-P\right)^{3}
$$

The critical pressure of water is $3203.6 \mathrm{psi}$. The homogeneous nucleation temperature is given in units of Fahrenheit. The effect of wall surface thermal properties are included by using a contact temperature correction.

$$
T_{\min , \mathrm{hn}}=T_{\mathrm{hn}}+\left(T_{\mathrm{hn}}-T_{l}\right)\left[\frac{\left(k_{p} C_{p}\right)_{l}}{\left(k_{p} C_{p}\right)_{w}}\right]^{1 / 2}
$$

The second method is by using Henry's modification of the Berenson correlation [88].

$$
T_{\text {min,henry }}=T_{B}+0.42\left(T_{B}-T_{l}\right)\left\{\left[\frac{\left(k_{p} C_{p}\right)_{l}}{\left(k_{p} C_{p}\right)_{w}}\right]^{1 / 2}\left[\frac{H_{\mathrm{fg}}}{C_{\mathrm{pw}}\left(T_{B}-T_{\mathrm{sat}}\right)}\right]\right\}^{0.6}
$$

Where,

$$
T_{B}=T_{\mathrm{sat}}+0.127 \frac{\rho_{v} H_{\mathrm{fg}}}{k_{v}}\left[\frac{g\left(\rho_{f}-\rho_{g}\right)}{\left(\rho_{f}+\rho_{g}\right)}\right]^{2 / 3}\left[\frac{g_{c} \sigma}{g\left(\rho_{f}-\rho_{g}\right)}\right]^{1 / 2}\left[\frac{\mu_{v}}{g\left(\rho_{f}-\rho_{g}\right)}\right]^{1 / 3}
$$

To summarize, the minimum film boiling temperature for heated surfaces is selected using the following criteria: 


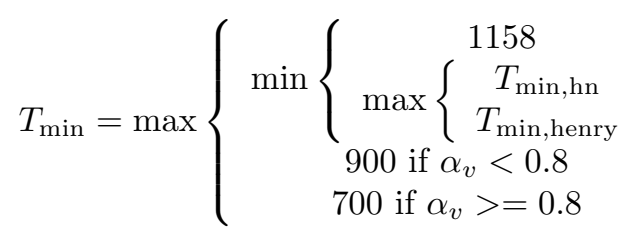

\subsubsection{Wall Heat Transfer Closure Term}

Closure terms are passed to the energy equations - wall heat transfer to the liquid phase is passed to the liquid energy equation and wall heat transfer to the vapor phase is passed to the vapor energy equation. The two closure terms are calculated as:

$$
\begin{gathered}
Q_{w v}=h_{v} A_{w}\left(T_{w}-T_{v}\right) \\
Q_{w l}=h_{l} A_{w}\left(T_{w}-T_{l}\right)
\end{gathered}
$$

The $h_{l}$ and $h_{v}$ terms are calculated from the liquid and vapor boiling regime heat transfer coefficients to ensure numerical stability. Additionally, the $h_{l}$ term is actually comprised of two different components: sensible heat transfer between the wall and liquid and latent heat transfer resulting from vaporization of the liquid.

First, the $h_{v}$ term is calculated from the vapor heat transfer coefficient, $h_{w v}$, as follows:

$$
h_{v}=\left[F_{v} h_{w v}\right]^{0.2}\left[\max \left\{\begin{array}{c}
h_{v}^{n} \\
1.0
\end{array}\right\}^{0.8}\right]
$$

$h_{v}^{n}$ is the old time step vapor heat transfer coefficient. The new time vapor heat transfer coefficient is weighted to the old time value to ensure a slower transition and numerical stability. $h_{w v}$ is the wall-to-vapor heat transfer coefficient, which is the boiling regime dependent heat transfer coefficient (calculation of which is further discussed later in this section). $F_{v}$ is a damping factor which diminishes the vapor heat transfer coefficient if the vapor phase is depleted. It is calculated as follows:

$$
F_{v}=\left\{\begin{array}{cl}
1.0 & \text { if } \alpha_{v}>=0.05 \\
\max \left\{\begin{array}{cl}
\frac{\alpha_{v}-0.01}{0.04} \\
0.0
\end{array}\right. & \text { if } \alpha_{v}<0.05
\end{array}\right.
$$

Second, the liquid heat transfer coefficient also multiplies by a damping factor and is weighted to the old time value.

$$
h_{l}^{*}=\left[F_{l}\left(h_{w l}+h_{w b}\right)\right]^{0.2}\left[\max \left\{\begin{array}{l}
h_{l}^{n} \\
0.1
\end{array}\right\}\right]^{0.8}
$$


The two components of the liquid heat transfer coefficient, $h_{w l}$ and $h_{w b}$, are summed together in Equation 6.107. The damping factor, $F_{l}$, is defined as follows:

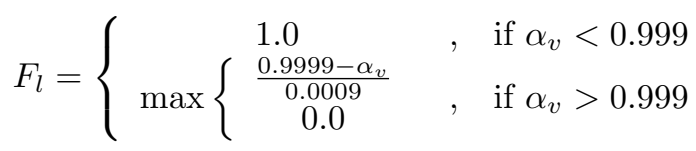

The sensible and latent heat transfer coefficients, $h_{w l}$ and $h_{w b}$, of Equation 6.107 are boiling regime dependent and are further discussed in the following sub-sections. The $h_{l}^{*}$ term of Equation 6.107 has the asterisk subscript because it is further modified to aid in numerical stability.

In the event of nucleate boiling, the heat flux is highly dependent on surface temperature, $T_{w}$. During the conduction equation solution, the surface temperature is highly dependent on the heat flux. Explicitly using the old time step heat flux in calculating the new time step wall temperature can lead to an oscillation in the calculation of $T_{w}$ throughout the transient. Therefore, when in the nucleate boiling regime, the wall temperature and heat flux calculations are implicitly coupled. Such that the solution is non-iterative, this is done by using the change of the heat transfer coefficient with respect to $T_{w}$ when calculating $h_{l}$.

$$
h_{l}=h_{l}^{*}+\left(\frac{d h}{d T_{w}}\right)\left(T_{w}-T_{w}^{n}\right)
$$

The linearized derivative of heat transfer coefficient with respect to wall temperature is calculated as:

$$
\frac{d h}{d T_{w}}=\max \left\{\operatorname { m i n } \left\{\begin{array}{c}
\frac{d h_{\mathrm{nb}}}{d T_{w}} \\
\frac{h_{l}^{*}}{\left(T_{w}-T_{l}\right)} \\
0.0
\end{array}\right.\right.
$$

$d h_{\mathrm{nb}} / d T$ is defined using the subcooled boiling/nucleate boiling correlation and is further discussed in section 6.6.6. With the $h_{l}$ and $h_{v}$ terms completely defined, the heat flux to each phase can be calculated.

The above information can be used in determining the ratio of latent heat transfer to total heat transfer. This ratio will be later useful for determining the vapor generation caused by subcooled boiling. This ratio is simply defined as:

$$
F_{\Gamma}=\frac{h_{w b}\left(T_{w}-T_{\mathrm{sat}}\right)}{h_{w l}\left(T_{w}-T_{l}\right)+h_{w b}\left(T_{w}-T_{\mathrm{sat}}\right)}
$$

Multiplying this ratio by the total liquid heat transfer rate yields the heat transfer due to subcooled boiling.

$$
Q_{b}^{*}=F_{\Gamma} h_{l} A_{w}\left(T_{w}-T_{l}\right)
$$

This term is also damped for numerical stability. 


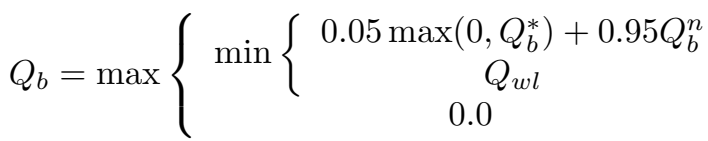

Recall that $Q_{w l}$ was defined in Equation 6.104 .

\subsubsection{Single-Phase Vapor Convection}

The flow is assumed to be in the single-phase vapor regime when void fraction is greater than 0.999 . In that case, three correlations are used to determine the heat transfer coefficient. These are the Dittus-Boelter correlation [89], the Wong and Hochreiter correlation for turbulent forced convection [90], and a constant Nusselt number value for laminar forced convection. To make for a seamless transition between the heat transfer coefficients predicted by different models, the maximum of the three correlations is selected if $\mathrm{CTF}$ determines that the boiling regime is single-phase vapor.

$$
h_{w v}=\max \left\{\begin{array}{c}
h_{w v, \mathrm{DB}} \\
h_{w v, \mathrm{WH}} \\
h_{w v, \mathrm{lam}}
\end{array}\right.
$$

First, the simplest model of the three is discussed. The heat transfer coefficient is determined assuming a constant Nusselt number of 10 .

$$
h_{w v, \operatorname{lam}}=10 \frac{k_{v}}{D_{h}}
$$

$k_{v}$ is the conductivity of vapor and $D_{h}$ is the hydraulic diameter of the channel.

The Dittus-Boelter correlation was originally developed for turbulent flow in smooth tubes inside of automobile radiators, but has found use for many turbulent flow modeling scenarios. The heat transfer coefficient is calculated as follows:

$$
h_{w v, \mathrm{DB}}=0.023 \frac{k_{v}}{D_{h}} \operatorname{Re}^{0.8} \operatorname{Pr}^{n}
$$

For heating, $n=0.4$ and for cooling, $n=0.3$. The fluid properties are evaluated at the mean film temperature.

The Wong and Hochreiter correlation was developed by applying a linear regression fit to experimental data obtained from rod bundle heat transfer tests performed on a 17x17 array of rods. The correlation is as follows:

$$
h_{w v, \mathrm{WH}}=0.07907 \frac{k_{v}}{D_{h}} \operatorname{Re}^{0.6774} \operatorname{Pr}_{v}^{0.333}
$$


Grid impact on heat transfer, if grids are present in the flow field, is modeled by multiplying the heat transfer coefficient by a grid effect factor, as follows:

$$
h_{w v}=F_{\text {grid }} h_{w v}
$$

\subsubsection{Single-Phase Liquid Heat Transfer}

The flow is in the single-phase liquid heat transfer regime if the liquid void fraction, $\alpha_{l}$, is greater than 0.001 and if no other heat transfer regimes are determined to exist. Single-phase liquid heat transfer coefficients are calculated for the case of laminar flow using a model recommended by Sparrow et al. [91] (Equation 6.119) and for the case of turbulent flow using Dittus-Boelter [89] (Equation 6.116, with liquid properties used instead of vapor properties). The maximum of these two calculations is taken as the liquid single-phase heat transfer coefficient.

$$
h_{w l, \operatorname{lam}}=7.86 \frac{k_{l}}{D_{h}}
$$

If liquid void fraction is below 0.001, the flow will be considered deficient of liquid and the single-phase vapor heat transfer coefficient, as calculated in Section 6.6.3, will be used and $h_{w l}$ will be set to zero.

\subsubsection{Subcooled Nucleate Boiling}

The subcooled nucleate boiling regime is encountered when small bubbles begin to form on the heated surface. Liquid near the heated wall must be at least slightly superheated for vapor to form; however, in the early stages of subcooled nucleate boiling, the bulk fluid temperature will be lower than the saturation temperature. Since the bulk fluid temperature isn't high enough to support the existence of void, bubbles that form at the wall will generally condense soon after they form which results in the existence of a very small void fraction.

In the later stages of subcooled nucleate boiling, as the bulk temperature increases, bubbles may grow and detach from the wall where they then later condense in the liquid. The bulk fluid temperature is still too low to support the bubbles' existence; however, this later region of subcooled nucleate boiling can have significant amounts of void in the flow.

Concerning heat transfer in this two-phase regime, there are three processes which will affect heat transfer from the wall to the liquid:

1. Forced convection to the liquid

2. Vapor generation at the wall

3. Condensation of bubbles at the wall 
CTF calculates the heat transfer coefficient in the subcooled nucleate boiling region using one of two correlations: the Chen correlation[86] or the Thom correlation[87]. The user is responsible for choosing the correlation they would like to use (see the User's Manual[5]). The convective and boiling heat transfer components are both considered in the original Chen and Thom papers. The condensation heat transfer effect was not considered in the references, but is instead added in after the fact in the code-it will be discussed after the two separate models.

Chen Correlation The Chen correlation calculates separately the heat transfer coefficients due to forced convection and vapor generation and adds them together. This may be summarized in equation form as:

$$
h_{\mathrm{chen}}=h_{\mathrm{fc}}+h_{\mathrm{nb}}
$$

Where the subscripts "fc" and "nb" stand for "forced convection" and "nucleate boiling", respectively. The nucleate boiling term is calculated using a Forster-Zuber type of pool boiling equation [92].

The forced convection heat transfer coefficient is calculated by means of a modified Dittus-Boelter type correlation, presented by Chen as follows:

$$
h_{\mathrm{fc}}=0.023 F_{\text {chen }}\left(\frac{k_{l}}{D_{h}}\right) \operatorname{Re}_{l}^{0.8} \operatorname{Pr}^{0.4}
$$

The $F_{\text {chen }}$ factor is used to account for the difference between two-phase and single-phase Reynolds number. Therefore, it will be equal to 1 in the subcooled boiling heat transfer regime and the Dittus-Boelter equation will remain un-modified. The $F_{\text {chen }}$ factor will be discussed later in the section on saturated boiling heat transfer (Section 6.6.6) where it will vary from 1. Additionally, the subcooled liquid properties will be used to determine the Reynolds number, Prandtl number, and liquid conductivity in the Dittus-Boelter equation.

The second component to the Chen model (Equation 6.120) is the nucleate boiling heat transfer coefficient. This is modeled by a Forster-Zuber [92] type of pool boiling equation. However, modification is made to account for the fact that the correlation is modeling forced convection boiling instead of pool boiling. The difference stems from the difference in boundary layer size between pool boiling and convective boiling. It can be shown that the product of growth rate and bubble radius is constant for a given superheat. The fluid temperature varies across the boundary layer, but because the pool boiling boundary layer is large compared to the bubble diameter, the superheat around the bubble can be assumed uniform. This is not true of the temperature around bubbles in forced convective boiling where the boundary layer is small and temperature gradients are steep. Therefore, Chen presented a suppression factor, $S_{\text {chen }}$ to account for this effect in the nucleate boiling heat transfer coefficient term. The nucleate boiling heat transfer correlation is as follows:

$$
h_{\mathrm{nb}}=0.00122 S_{\text {chen }}\left(\frac{k_{f}^{0.79} C p_{f}^{0.45} \rho_{f}^{0.49} g_{c}^{0.25}}{\sigma^{0.5} \mu_{f}^{0.29} H_{\mathrm{fg}}^{0.24} \rho_{g}^{0.24}}\right)\left(T_{w}-T_{f}\right)^{0.24}\left(P\left(T_{w}\right)-P\left(T_{\text {sat }}\right)\right)^{0.75}
$$

The boiling suppression factor, $S_{\text {chen }}$ is a function of the two-phase Reynolds number. 


$$
S_{\text {chen }}=\left\{\begin{array}{cl}
{\left[1+0.12 R e_{2 \Phi}^{1.14}\right]^{-1}} & \text { if } R e_{2 \Phi}<32.5 \\
{\left[1+0.42 R e_{2 \Phi}^{0.78}\right]^{-1}} & \text { if } 32.5<R e_{2 \Phi}<50.9 \\
0.1 & R e_{2 \Phi}>50.9
\end{array}\right.
$$

The two-phase Reynolds number is calculated using the Chen Reynolds number factor; however, recall that $F_{\text {chen }}$ will be 1 for the subcooled nucleate boiling region.

$$
R e_{2 \Phi}=\left(10^{-4}\right) R e_{l} F_{\text {chen }}^{1.25}
$$

Returning to the calculation of the nucleate boiling heat transfer coefficient (Equation 6.122), the terms $P\left(T_{w}\right)$ and $P\left(T_{\text {sat }}\right)$ represent the pressure at the wall temperature and the pressure at the saturation temperature. The difference between these pressures is approximated as follows:

$$
\begin{aligned}
\left(P\left(T_{w}\right)-P\left(T_{\mathrm{sat}}\right)\right) & =\left[\frac{5.4042 H_{\mathrm{fg}}}{\nu_{\mathrm{fg}}\left(T_{\mathrm{sat}}+460\right)}\right]\left(T_{w}-T_{\mathrm{sat}}\right)^{A}, \text { where } \\
A & =\frac{1.0306}{\left(\log _{10} P\right)^{0.017}}+\frac{0.0020632}{\left(\log _{10} P\right)^{1.087}} \max \left\{0.0,\left(T_{w}-T_{\mathrm{sat}}\right)-5.0\right\}
\end{aligned}
$$

Before completing the calculation of the necessary heat transfer coefficients, recall that during the overall discussion of the wall heat transfer closure term in Section 6.6.2 we made mention of the fact that, for the nucleate boiling regions, the heated surface temperature, $T_{w}$, and the wall heat transfer term were implicitly coupled by using the linearized derivative of heat transfer coefficient with respect to surface temperature. The derivative of heat transfer coefficient with respect to $T_{w}$ is calculated from the nucleate boiling heat transfer coefficient, presented in Equation 6.122.

$$
\begin{aligned}
\frac{d h_{\text {chen }}}{d T_{w}}= & {[0.24+0.75\{\text { chen } 1+\text { chen } 2\}] \frac{h_{\mathrm{nb}}}{T_{w}-T_{\mathrm{sat}}}+} \\
& \left\{\begin{array}{cc}
\frac{0.001547}{\left(\log _{10} P\right)^{1.087}} h_{\mathrm{chen}} \ln \left(T_{w}-T_{\mathrm{sat}}\right) & \text { if }\left(T_{w}-T_{\mathrm{sat}}\right)>5 \\
0.0 & \text { if }\left(T_{w}-T_{\mathrm{sat}}\right)<5
\end{array},\right. \text { where } \\
\text { chen } 1= & \frac{1.0306}{\left(\log _{10} P\right)^{0.017}} \text { and } \\
\text { chen2 }= & \frac{0.0020632}{\left(\log _{10} P\right)^{1.087}} \max \left\{0.0,\left(T_{w}-T_{\mathrm{sat}}\right)-5.0\right\}
\end{aligned}
$$

Thom Correlation Unlike Chen, the Thom correlation assumes that forced convection is negligible compared to nucleate boiling in this heat transfer regime. The boiling heat transfer coefficient was calculated using experimental data leading to forced convection and subcooled nucleate boiling in the test facility. With measured rod-surface temperatures, rod heat fluxes, and fluid temperatures, it was possible to develop a relationship between heat flux and rod surface temperature superheat $\left(\Delta T_{\text {sat }}=T_{\text {wall }}-T_{\text {sat }}\right)$. By considering the noted dependence of $\Delta T_{\text {sat }}$ on pressure, pressure was also included in the correlation. The Thom correlation, for heat flux due to subcooled boiling was derived as follows: 


$$
q_{\mathrm{nb}}^{\prime \prime}=\frac{\exp \left(\frac{2 P}{1260}\right)}{72^{2}} \Delta T_{\mathrm{sat}}^{2}
$$

The derivative of the heat transfer coefficient with respect to rod surface temperature must also be determined for Thom, as it is for Chen, in order to do the implicit coupling between the rod heat flux to the fluid and rod temperature. This was calculated using a numerical approximation with a step size of $1 \mathrm{~F}$ as follows:

$$
\frac{d H T C}{d T_{\mathrm{s}}}=\frac{q_{\mathrm{nb}}^{\prime \prime}\left(\Delta T_{\mathrm{sat}}+1\right)}{\max \left(1 \cdot 10^{-6}, \Delta T\right)+1}-\frac{q_{\mathrm{nb}}^{\prime \prime}\left(\Delta T_{\mathrm{sat}}\right)}{\max \left(1 \cdot 10^{-6}, \Delta T\right)}
$$

The temperature difference in the denominators will be $T_{\text {wall }}-T_{\text {liq }}$ for subcooled nucleated boiling.

Near-Wall Condensation Heat Transfer The near-wall condensation heat transfer is the last important term in the subcooled nucleate boiling regime. One of the subcooled nucleate boiling correlations will provide the overall heat transfer from the wall to the fluid, but in the subcooled boiling region, not all generated vapor will stay as vapor - much will inevitably condense back into liquid since the temperature of the bulk fluid is not high enough to support the existence of vapor. In reality, this is returning energy to the liquid phase through latent heat transfer. The condensation heat flux is needed to determine the ratio of latent to sensible heat transfer from the wall to the fluid.

To calculate the condensation heat flux, we use the correlation of Hancox-Nicoll[93], which gives the heat flux at the point where bubbles generated in the near-wall region collapse and re-condense back to liquid.

The condensation heat flux, $q_{\text {cond }}^{\prime \prime}$, is thus equal to the heat flux into the liquid considering the condensation of all near-wall bubbles, $q_{\mathrm{hn}}^{\prime \prime}$, by using the Hancox-Nicoll correlation, as follows:

$$
q_{\mathrm{cond}}^{\prime \prime}=q_{\mathrm{hn}}^{\prime \prime}=0.4\left(\frac{C p_{f} \mu_{f}}{D_{h}}\right)\left(\frac{G_{l} D_{h}}{\mu_{f}}\right)^{0.662}\left(T_{\mathrm{sat}}-T_{l}\right)
$$

Calculation of Heat Transfer Coefficient Terms With the three main heat transfer phenomenon discussed and defined, it is now possible to calculate the three heat transfer coefficients- $h_{w l}, h_{w b}$, and $h_{w v}$ - that will be needed in the CTF coupling between rods and coolant.

First, the wall heat transfer to vapor will be set to zero in CTF unless the void fraction is larger than 0.999. If $\alpha_{v}$ is greater than 0.999 then the vapor heat transfer coefficient is calculated as in Section 6.6.3 and the liquid heat transfer coefficients are set to zero.

For the latent and sensible heat transfer coefficients to the liquid, the fraction of heat that results in net vapor generation must be calculated. The heat flux predicted by Hancox-Nicoll is the total heat dissipation ability in the subcooled boiling region, which may be in excess of the actual wall heat flux. The single-phase heat flux (calculated from Dittus-Boelter) is subtracted from this term to get the excess heat removal ability in the subcooled boiling region. Then, this term is subtracted from the nucleate boiling heat flux predicted by the subcooled boiling model. This represents the heat flux that results in vapor genreation in the subcooled boiling region. This is divided by the total heat flux to determine the vapor generation fraction. 


$$
F_{\text {gam }}^{*}=\frac{h_{\mathrm{nb}}\left(T_{w}-T_{\mathrm{sat}}\right)-q_{\mathrm{cond}}^{\prime \prime}}{h_{\mathrm{nb}}\left(T_{w}-T_{\mathrm{sat}}\right)}
$$

The vapor that does escape the vapor layer near the wall will be replaced with subcooled liquid from the bulk. The wall layer will heat up this subcooled liquid before it can be boiled, so this will reduce further the ability of vapor generation. A model was proposed by Rouhani to capture this effect.

$$
\epsilon_{\Gamma}=\frac{h_{\mathrm{fg}}}{h_{\mathrm{fg}}+\left(h_{f}-h_{l}\right) \frac{\rho_{f}}{\rho_{g}}}
$$

This term is multiplied by Equation 6.130 if the heat transfer regime is subcooled boiling. In bulk boiling, the heat flux to vapor is left unperturbed because the bulk coolant is now saturated.

This leads us to the calculation of the latent heat transfer to the liquid. We simply multiply the total boiling heat transfer term by the fraction of heat transfer that results in stable vapor production.

$$
q_{w b, \mathrm{scb}}^{\prime \prime}=F_{\mathrm{gam}} q_{\mathrm{nb}}^{\prime \prime}
$$

Since we are concerned with the heat transfer coefficient, we divide through by $\left(T_{w}-T_{\text {sat }}\right)$.

$$
h_{w b, \mathrm{scb}}=F_{\mathrm{gam}} h_{\mathrm{nb}}
$$

The energy that condenses out of the vapor phase is added to the sensible heat transfer term alongside the single-phase heat transfer term.

$$
q_{w l, \mathrm{scb}}^{\prime \prime}=q_{\mathrm{fc}}^{\prime \prime}+\left(1-F_{\mathrm{gam}}\right) q_{\mathrm{nb}}^{\prime \prime}
$$

Once again, we divide this through by the temperature difference to obtain the heat transfer coefficient - this time the temperature difference is $T_{w}-T_{l}$.

$$
h_{w l, \mathrm{scb}}=h_{\mathrm{fc}}+\left(1-F_{\mathrm{gam}}\right) h_{\mathrm{nb}} \frac{T_{w}-T_{\mathrm{sat}}}{T_{w}-T_{l}}
$$

\subsubsection{Saturated Nucleate Boiling}

In the saturated nucleate boiling regime, the bulk fluid has reached the saturation temperature. The heat transfer mechanisms still include forced convection and nucleate boiling, but the near-wall condensation term is no longer of issue like it was in the subcooled nucleate boiling region.

The correlations from the subcooled boiling regime are extended and used in the saturated boiling regime as well. The Chen correlation was actually derived from saturated boiling data while Thom was developed 
for subcooled nucleate boiling data, though it is common to extend Thom's use to saturated boiling[94]. Since Chen was developed from low-pressure data (i.e., 1000 psi compared to the pressure of 750-2000 psi for Thom), it has been observed to under-perform Thom for test conditions that reflect those of prototypical PWRs[94].

Chen Correlation The forced convection component is revisited. The Dittus-Boelter type equation is still used (re-stated here for convenience).

$$
h_{\mathrm{fc}}=0.023 F_{\text {chen }}\left(\frac{k_{f}}{D_{h}}\right) R e_{l}^{0.8} \operatorname{Pr}^{0.4}
$$

However, the conductivity, Reynolds number, and Prandtl number are calculated with consideration that a two-phase fluid exists instead of a single phase one. Additionally, the forced convection term, $F_{\text {chen }}$, is no longer restricted to a value of 1.0 as it was in the case of subcooled nucleate boiling.

Note that the conductivity is that of a saturated liquid. Accounting for the fact that the fluid is two-phase, $R e_{l}$ is calculated as:

$$
R e_{l}=\frac{G_{l} D_{h}}{\mu_{f}}
$$

The $F_{\text {chen }}$ factor is a function of the Martinelli factor, $\chi_{T T}$.

$$
F_{\text {chen }}=\left\{\begin{array}{cc}
1.0 & \text { if } \chi_{T T}^{-1}<0.1 \\
2.34\left(\chi_{T T}^{-1}+0.213\right)^{0.736} & \text { if } \chi_{T T}^{-1}>0.1
\end{array}\right.
$$

The Martinelli parameter is the square root of the ratio of liquid friction pressure drop to vapor friction pressure drop.

$$
\chi^{2}=\frac{\left(\frac{d P}{d Z}\right)_{\text {fric }}^{l}}{\left(\frac{d P}{d Z}\right)_{\text {fric }}^{v}}
$$

Equation 6.138 can be re-written in terms of the phase viscosities and densities and quality. The inverse of the Martinelli parameter becomes:

$$
\chi_{T T}^{-1}=\left(\frac{x}{1-x}\right)^{0.9}\left(\frac{\rho_{f}}{\rho_{g}}\right)^{0.5}\left(\frac{\mu_{g}}{\mu_{f}}\right)^{0.1}
$$

In the code, this value is limited to a maximum value of 100 . 


$$
\chi_{T T}^{-1}=\min \left[\chi_{T T}^{-1}, 100\right]
$$

The quality, $x$, is calculated as:

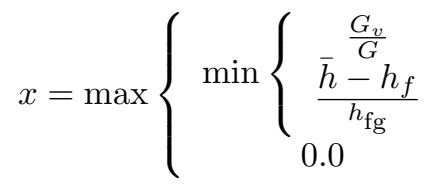

The mixture enthalpy, $\bar{h}$, is a density weighted enthalpy. It is calculated as follows:

$$
\bar{h}=\frac{\alpha_{v} \rho_{v} h_{v}+\left(\alpha_{l}+\alpha_{e}\right) \rho_{l} h_{l}}{\alpha_{v} \rho_{v}+\left(\alpha_{l}+\alpha_{e}\right) \rho_{l}}
$$

The boiling heat transfer coefficient is still calculated using the modified Foster-Zuber model that was shown in Equation 6.122. However, the two-phase Reynolds number is calculated differently - it is multiplied by the $F_{\text {chen }}$ factor to account for the increase caused by boiling.

$$
R e_{2 \Phi}=10^{-4} R e_{l} F_{\text {chen }}^{1.25}
$$

This is used in the calculation of the boiling suppression factor, $S_{\text {chen }}$, in Equation 6.123.

Finally, the derivative of the heat transfer coefficient with respect to temperature is calculated as it was for the subcooled nucleate boiling regime.

Thom Correlation The Thom correlation is completely unmodified from its form in the subcooled nucleate boiling regime with Equation 6.127.

Like for the subcooled boiling region, $d H T C / d T$ is calculated; however, the denominator of the numerical approximation, $\Delta T$, will be defined as $T_{\text {wall }}-T_{\mathrm{f}}$ (the wall temperature minus the liquid saturation temperature).

Calculation of Heat Transfer Coefficient Terms Calculation of the heat transfer coefficient terms is simplified without the need for consideration of the near-wall condensation term. The latent heat transfer coefficient sums the forced convection and nucleate boiling terms.

$$
h_{w b}=h_{\mathrm{fc}}+h_{\mathrm{nb}} F_{B}
$$

Note that, a ramp, $F_{B}$, is added in the code to ensure a smooth decrease in the boiling term as dryout occurs. 


$$
F_{B}=\min \left\{\begin{array}{c}
416.7\left(\alpha_{l}-0.0001\right) \\
1.0
\end{array}\right.
$$

The sensible heat transfer coefficient, $h_{w b}$, is calculated as it would be for single-phase liquid-by choosing the maximum of the heat transfer coefficients predicted by the laminar flow model (Equation 6.119) or by the turbulent, Dittus-Boelter model (Equation 6.116).

The heat transfer coefficient to vapor, $h_{w v}$, is calculated as it was for the subcooled nucleate boiling region. It is zero unless void is above 0.999 , in which case it is calculated as it was for single-phase vapor in Section 6.6 .3

\subsubsection{Transition Boiling}

The transition boiling heat flux is considered to be a combination of four different heat transfer effects. There is convective heat transfer to the single-phase vapor, boiling heat transfer to the liquid, radiation heat transfer to the liquid, and radiation heat transfer to the vapor. This is summarized in equation form:

$$
q_{t b}^{\prime \prime}=q_{w v}^{\prime \prime}+q_{w b}^{\prime \prime}+q_{r w v}^{\prime \prime}+q_{r w l}^{\prime \prime}
$$

The single-phase vapor convection, $q_{w v}^{\prime \prime}$, is calculated as was described previously in Section 6.6.3. Any heat transfer to liquid in contact with the heated surface results in boiling of the liquid - the fraction of liquid that contacts the surface experiences a heat flux equal to the critical heat flux, calculated in Section 6.5.

$$
q_{w b}^{\prime \prime}=F_{w} q_{\mathrm{chf}}^{\prime \prime} * R_{\mathrm{dryout}}
$$

The fraction of liquid in contact with the wall was given by Bjornard and Griffith [73] as a function of wall temperature, minimum film boiling temperature, and critical heat flux temperature. The wall fraction correlation provided good agreement with data given by Groenveld and Fung[95] and McCreery et al. [96].

$$
F_{w}=\left(\frac{T_{w}-T_{\min }}{T_{\mathrm{chf}}-T_{\min }}\right)^{2}
$$

The ramp, $R_{\text {dryout }}$, was added to diminish the boiling heat flux as the liquid phase is depleted. It is defined as follows:

$$
R_{\text {dryout }}=\min \left\{\begin{array}{c}
1.0 \\
\max \left\{\begin{array}{c}
0.0 \\
400\left(\alpha_{l}-0.0025\right)
\end{array}\right.
\end{array}\right.
$$

The boiling heat transfer coefficient, is then calculated as: 


$$
h_{w b}=F_{\mathrm{scb}} \frac{q_{w b}^{\prime \prime}}{T_{w}-T_{f}}
$$

Where $F_{\mathrm{scb}}$ is the subcooled boiling modification factor, which is defined in Equation 6.151 (repeated below for convenience). The subcooled boiling modification factor is a ramp that prevents sharp discontinuities in vapor generation as the liquid subcooling becomes small.

$$
F_{\mathrm{scb}}=\min \left\{\operatorname { m a x } \left\{\begin{array}{c}
1.0 \\
\frac{h_{\mathrm{fg}}}{h_{\mathrm{fg}}+\left(h_{f}-h_{l}\right) \frac{\rho_{f}}{\rho_{g}}} \\
0.01
\end{array}\right.\right.
$$

This ramp may prevent a portion of liquid from turning to vapor in the code. The heat flux entering the liquid is conserved by adding the portion that doesn't turn to vapor to the liquid heat transfer coefficient.

$$
h_{w l}=h_{w l}+\left(1-F_{\mathrm{scb}}\right) \frac{q_{w b}^{\prime \prime}}{\max \left(1, T_{w}-T_{l}\right)}
$$

The radiative heat transfer to liquid and vapor is discussed separately in Section 6.6.10 due to its importance to all post-CHF heat transfer regimes. The heat transfer coefficients resulting from radiative heat transfer are simply added to the respective phase heat transfer coefficients already calculated.

$$
\begin{aligned}
& h_{w v}=h_{w v}+h_{r w v}, \text { and } \\
& h_{w l}=h_{w l}+h_{r w l}
\end{aligned}
$$

\subsubsection{Inverted Annular Film Boiling}

The inverted annular film boiling heat transfer coefficients are calculated if void is between 0.4 and 0.95 and if the inverted annular film boiling heat flux is greater than the dispersed flow film boiling heat flux. Therefore, the dispersed flow film boiling is calculated first in the CTF source in order to compare to the inverted annular film boiling heat flux.

The inverted annular film boiling heat flux is calculated with use of the modified Bromley correlation [97].

$$
h_{\text {brom }}=0.62\left(\frac{D_{h}}{\lambda}\right)^{0.172}\left[\frac{k_{g}^{3} \rho_{g}\left(\rho_{f}-\rho_{g}\right) H_{\mathrm{fg}} g}{D_{h} \mu_{g}\left(T_{w}-T_{\mathrm{sat}}\right)}\right]^{1 / 4}
$$

The critical wavelength, $\lambda$, is give as: 


$$
\lambda=2 \pi \sqrt{\frac{g_{c} \sigma}{g\left(\rho_{f}-\rho_{g}\right)}}
$$

The heat flux in the inverted annular film boiling regime can then be obtained by multiplying the heat transfer coefficient by the wall superheat.

$$
q_{\mathrm{brom}}^{\prime \prime}=h_{\mathrm{brom}}\left(T_{w}-T_{\mathrm{sat}}\right)
$$

The inverted annular film boiling heat flux needs to be compared to the dispersed flow film boiling heat flux. The maximum one will define the heat transfer regime - if $q_{d f f b}^{\prime \prime}$ (plus heat flux caused by droplets impinging on the heated surface) is larger, the regime will be dispersed flow film boiling and if $q_{\text {iafb }}^{\prime \prime}$ (plus heat flux caused by liquid boiling on the surface) is larger, the regime will be inverted annular film boiling.

If it is found that the inverted annular film boiling heat flux is larger than the dispersed flow film boiling heat flux, the inverted annular film boiling heat flux calculations continue. First, because the Bromley correlation, Equation 6.154, was obtained from experimental observation, it includes all of the heat transfer effects; however, we need to separate those effects for the calculation of the individual heat transfer coefficient terms. Therefore, we begin by subtracting off the vapor heat transfer terms (both forced convection and radiation heat transfer).

$$
q_{w l}^{\prime \prime}=q_{\mathrm{brom}}^{\prime \prime}-h_{\mathrm{spv}}\left(T_{w}-T_{v}\right)-h_{w v r}\left(T_{w}-T_{v}\right)
$$

To prevent the liquid heat flux from being negative, the maximum of $q_{w l}^{\prime \prime}$ or 0.0 is chosen. The heat flux is then partitioned into liquid and vapor components. If the vapor is not superheated, then the liquid heat flux, $q_{\text {liq }}^{\prime \prime}$, is equal to $q_{w l}^{\prime \prime}$ from Equation 6.157 and the vapor heat flux, $q_{\text {vap }}^{\prime \prime}$ is zero. For the event that the vapor is superheated, the effects vapor void fraction and amount of superheat are taken into account using a multiplier.

$$
\begin{aligned}
& q_{l}^{\prime \prime}=\left(1-F_{i a f b}\right) q_{w l}^{\prime \prime} \\
& q_{v}^{\prime \prime}=F_{i a f b} q_{w l}^{\prime \prime}
\end{aligned}
$$

Where,

$$
F_{i a f b}=1.11 \alpha_{v} \sqrt{\frac{T_{w}-T_{v}}{T_{w}-T_{\mathrm{sat}}}}
$$

If the void is below 0.4 , then the regime is determined to be completely inverted annular film boiling. The boiling heat transfer coefficient is calculated from the liquid heat flux calculated above and the modified annular film boiling heat flux. 


$$
h_{w b}=\frac{q_{\mathrm{liq}}^{\prime \prime}+q_{t b}^{\prime \prime}}{T_{w}-T_{f}}
$$

$q_{t b}^{\prime \prime}$ is the modified annular film boiling heat flux. To derive it, we start from Equation 6.69, which was presented in the section on CHF (Section 6.5). The modified Zuber equation is restated here for convenience:

$$
q_{\mathrm{chf}}^{\prime \prime}=0.15\left(1-\alpha_{v}\right) H_{\mathrm{fg}} \rho_{g}^{0.5}\left[g_{c} g \sigma\left(\rho_{f}-\rho_{g}\right)\right]^{0.25}
$$

Since only a very small portion of the liquid will be in contact with the heated surface, the modified Zuber equation is multiplied by the liquid void fraction, $\alpha_{l}$, and by a liquid contact effectiveness multiplier, $\epsilon_{\mathrm{wet}}$, which was defined by Ganic and Rohsenow [98].

$$
\epsilon_{\mathrm{wet}}=\exp \left[1-\left(\frac{T_{w}}{T_{f}}\right)^{2}\right]
$$

Thus, $q_{t b}^{\prime \prime}$ of Equation 6.160 is:

$$
q_{t b}^{\prime \prime}=q_{\mathrm{chf}}^{\prime \prime} \alpha_{l} \epsilon_{\mathrm{wet}}
$$

The vapor heat transfer coefficient is calculated as the sum of the single-phase vapor heat transfer coefficient (derived in Section 6.6.3), the radiation heat transfer coefficient to vapor (derived in Section 6.6.10), and the vapor heat flux obtained from the Bromley correlation.

$$
h_{w v}=h_{\mathrm{spv}}+h_{r w v}+\frac{q_{\mathrm{vap}}^{\prime \prime}}{T_{w}-T_{v}}
$$

The liquid heat transfer coefficient, $h_{w l}$, only includes the effect of wall to droplet radiative heat transfer, which is discussed in Section 6.6.10.

If the void fraction was found to be above 0.4 , the heat flux is ramped between that of inverted annular film boiling heat flux and that of dispersed flow film boiling heat flux. First, the ramp is defined:

$$
R_{i a d f}=\frac{0.95-\alpha_{v}}{0.5}
$$

Note that at a void fraction of 0.4 , the ramp will be slightly larger than 1 and as it grows to 0.95 , the ramp will shrink to zero. The boiling heat transfer coefficient, $h_{w b}$, is calculated as the maximum of either the dispersed flow film boiling heat transfer that results from droplets impinging on the heated surface or the annular film boiling heat flux of the inverted annular film boiling region. The former, $q_{d e, d f f b}^{\prime \prime}$ or deentrainment boiling heat flux, is calculated in Section 6.6.9 on dispersed flow film boiling. The latter, $q_{t b}^{\prime \prime}$, is presented in Equation 6.162. The boiling heat transfer coefficient is calculated as follows: 


$$
h_{w b}^{*}=\max \left[\frac{q_{d e, d f f b}^{\prime \prime}}{T_{w}-T_{f}}, R_{i a d f} \frac{q_{t b}^{\prime \prime}}{T_{w}-T_{\text {sat }}}\right]
$$

The liquid portion of the Bromley correlation is then added to the result of this equation.

$$
h_{w b}=h_{w b}^{*}+R_{\text {iadf }} \frac{q_{\text {liq }}^{\prime \prime}}{T_{w}-T_{\text {sat }}}
$$

The vapor heat transfer coefficient is calculated as the sum of the single-phase vapor heat transfer coefficient (derived in Section 6.6.3), the radiation heat transfer coefficient to vapor (derived in Section 6.6.10), and the vapor heat flux obtained from the Bromley correlation.

$$
h_{w v}=h_{\mathrm{spv}}+h_{r w v}+R_{i a d f} \frac{q_{\mathrm{vap}}^{\prime \prime}}{T_{w}-T_{v}}
$$

Similar to before, the liquid heat transfer coefficient, $h_{w l}$, only includes effects of liquid radiative heat transfer, which is calculated in Section 6.6.10.

\subsubsection{Dispersed Flow Film Boiling}

The dispersed flow film boiling heat transfer regime will be selected when the wall temperature is above the CHF temperature and the minimum film boiling temperature and when the void fraction is above 0.95 . The dispersed flow film boiling heat transfer regime can also be selected if void is below 0.95 if the dispersed flow film boiling heat flux is greater than the inverted annular film boiling heat flux. As was discussed in Section 6.6.8, the heat flux will be linearly interpolated between the inverted annular film boiling and dispersed flow film boiling regimes if the void is between 0.4 and 0.95 .

The dispersed flow film boiling heat transfer term is a combination of convective heat transfer to vapor, radiative heat transfer to vapor, radiative heat transfer to liquid (droplets), and boiling heat transfer occurring from the collision of droplets with the heated rods. The vapor convective heat transfer term was calculated in Section 6.6.3 and the radiative heat transfer terms are calculated in Section 6.6.10. The boiling heat transfer term is calculated as a maximum of the CHF term and a droplet de-entrainment heat flux term.

$$
q_{b}^{\prime \prime}=\max \left[q_{\mathrm{chf}}^{\prime \prime}, q_{d e}^{\prime \prime}\right]
$$

The de-entrainment boiling heat flux is calculated using a de-entrainment coefficient which is multiplied by total droplet mass flux and the latent heat of vaporization.

$$
q_{d e}^{\prime \prime}=h_{\mathrm{fg}} C_{d e} G_{e}
$$

Both axial and transverse components of velocity are included in the droplet mass flux term, $G_{e}$, which has units of $\mathrm{lbm} / \mathrm{hr}_{\mathrm{ft}}{ }^{2}$. Like for the inverted annular film boiling heat transfer, the boiling heat transfer coefficient 
is calculated considering that only a fraction of droplets contact the heated surface due to the high wall superheat. The same wall-wetting fraction that was used for inverted annular film boiling is used here, repeated below:

$$
\epsilon_{\mathrm{wet}}=\exp \left[1-\left(\frac{T_{w}}{T_{f}}\right)^{2}\right]
$$

The boiling heat transfer coefficient is then calculated as follows:

$$
h_{w b}=\frac{q_{d e}^{\prime \prime} \epsilon_{\mathrm{wet}}}{T_{w}-T_{\mathrm{sat}}}
$$

\subsubsection{Radiative Heat Transfer}

The radiative heat transfer models used in CTF were developed by Sun, Gonzalez, and Tien [99]. The basis is that the wall, liquid, vapor, and droplets can all be treated as single nodes between which radiative heat transfer takes place so long as the flow is assumed to be optically thin. The heat transfer between bodies can be modeled using a gray body factor, $F$, in conjunction with the temperature gradient between bodies ( $T_{w}$ is the wall temperature and $T_{b}$ is the temperature of the body that radiative heat transfer flows to).

$$
q_{r}^{\prime \prime}=F\left(T_{w}^{4}-T_{b}^{4}\right)
$$

The heat transfer coefficients for the radiative heat transfer can then be defined. Since the temperature, emissivity, and surface area differs for different bodies (i.e. drops, liquid core, vapor), the different fields will have different gray body factors and temperatures.

$$
\begin{aligned}
& h_{r w v}=F_{w v} \frac{T_{w}^{4}-T_{v}^{4}}{T_{w}-T_{v}} \\
& h_{r w e}=F_{w e} \frac{T_{w}^{4}-T_{\mathrm{sat}}^{4}}{T_{w}-T_{l}} \\
& h_{r w l}=F_{w l} \frac{T_{w}^{4}-T_{\mathrm{sat}}^{4}}{T_{w}-T_{l}}
\end{aligned}
$$

The gray body factors are defined as a function of the Stefan-Boltzman constant, $\sigma_{s b}$, the emissivity of the surfaces of interest, and the surface areas of the bodies of interest. Variables, $R_{1}, R_{2}$, and $R_{3}$ are introduced to simplify the upcoming presentation of the gray body factors. The variables are later defined. The gray body factors are calculated as follows: 


$$
\begin{aligned}
& F_{w v}=\frac{\sigma_{s b}}{R_{1}\left(1+\frac{R_{3}}{R_{1}}+\frac{R_{3}}{R_{2}}\right)} \\
& F_{w e}=\min \left\{\begin{array}{c}
\frac{\sigma_{s b}}{R_{2}\left(1+\frac{R_{3}}{R_{1}}+\frac{R_{3}}{R_{2}}\right)} \\
F_{w l}
\end{array}\right. \\
& F_{w l}=\frac{\sigma_{s b}}{\frac{1}{\epsilon_{l}}+\frac{A_{w}}{A_{l}}\left(\frac{1}{\epsilon_{w}}-1\right)}
\end{aligned}
$$

The Stefan-Boltzman constant is $1.713 \times 10^{-9} \mathrm{BTU} / \mathrm{hr} \cdot \mathrm{ft}^{2} \cdot{ }^{\circ} \mathrm{R}^{4}$. A simplification is made for the liquid gray body factor. The only instance of radiation from the wall to the liquid is when inverted annular film flow exists - in this case, radiative heat transfer takes place between the wall and the column of water in the sub-channel center. For this case, the liquid emissivity, $\epsilon_{l}$, is assumed to be 1.0 , the wall emissivity, $\epsilon_{w}$, is assumed to be 0.7 , and the vapor void is assumed to be 0.65 . The ratio of the wall to liquid surface area can be reduced to a function of liquid void fraction if we consider concentric cylinder geometry:

$$
\frac{A_{w}}{A_{l}}=\frac{D_{h}}{D_{l}}=\frac{1}{\sqrt{\alpha_{l}}}
$$

Making these substitutions, the liquid grey body factor becomes a constant of $7.71 \times 10^{-10}$. To define the vapor and droplet grey body factors, we define the $R$ variables:

$$
\begin{aligned}
& R_{1}=\frac{1-\epsilon_{v}}{\epsilon_{v}\left(1-\epsilon_{v} \epsilon_{e}\right)} \\
& R_{2}=\frac{1-\epsilon_{e}}{\epsilon_{e}\left(1-\epsilon_{v} \epsilon_{e}\right)} \\
& R_{3}=\frac{1}{1-\epsilon_{v} \epsilon_{e}}+\frac{1-\epsilon_{w}}{\epsilon_{w}}
\end{aligned}
$$

The droplet and vapor emissivities are a function of the beam length and droplet and vapor absorption coefficients.

$$
\begin{aligned}
& \epsilon_{e}=1-\exp \left(-0.85 \alpha_{e a} L_{b}\right) \\
& \epsilon_{v}=1-\exp \left(-0.85 \alpha_{v a} L_{b}\right)
\end{aligned}
$$

The beam length is assumed equal to the hydraulic diameter, $D_{h}$. The droplet absorption coefficient is a function of droplet surface area and absorption efficiency, $P s i_{d}$. 


$$
\alpha_{e a}=\Psi N_{d} \frac{\pi D_{d}^{2}}{4}
$$

The droplet diameter is $D_{d}$ and the number of droplets is $N_{d}$. The absorption efficiency, $\Psi_{d}$, has a value of 0.74 for drops having diameters between 0.004 and 0.08 in. The number of droplets can be expressed as a function of void fraction.

$$
N_{d}=\frac{6\left(1-\alpha_{v}\right)}{\pi D_{d}^{3}}
$$

The droplet absorption coefficient can then be reduced:

$$
\alpha_{e a}=1.11 \frac{\left(1-\alpha_{v}\right)}{D_{d}}
$$

The vapor absorption coefficient is a function of absolute fluid pressure, $P$, and vapor temperature.

$$
\alpha_{v a}=\frac{P}{14.7}\left[5.6\left(\frac{1000}{T_{v}+460}\right)^{2}-0.3\left(\frac{1000}{T_{v}+460}\right)^{4}\right]
$$

The droplet and vapor emissivities are constrained to values between 0.001 and 0.75 .

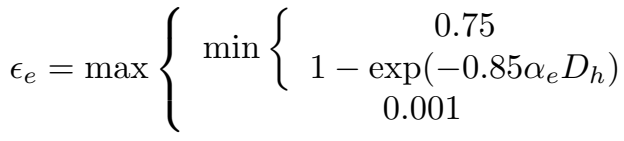

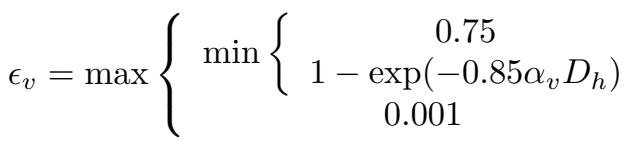

\subsubsection{Grid Re-Wet Model}

During accident conditions, where there is high vapor content in the flow and rod cooling is poor, the presence of spacer grids can lead to improved fuel rod cooling. The reason being that, since grids have no internal heat source, they will be the first to quench-impinging droplet will be likely to form a film. The presence of a film on the grids will lead to grid cooling as well as de-superheating of the vapor that flows past the grids. The de-superheated vapor will improve fuel rod cooling downstream. Furthermore, radiative heat transfer from the rods to the grids and radiative heat transfer from the vapor to the grids will be increased.

When grids are quenched, there will be a dry region and a wet region. The heat transfer to the grid will depend on whether the region is wet or dry. Heat balances are performed for each region of the grid, which 
are described in the following sections. The grid quench front model is also described, which will depend on the evaporation rate of the grid film as well as the availability of water from impinging droplets. The grid quench front calculations are performed after the heat balances - the initial grid quench front location is supplied by user input.

\subsubsection{Dry Region Heat Balance}

The heat balance in the dry region includes the radiative heat transfer to the grid from the fuel rods and vapor, the convective heat flux from the grid to the vapor, and the heat flux from the grid due to droplets impinging on its surface. This is summarized in equation form.

$$
\rho_{g} C_{p, g} \frac{\partial T}{\partial t}=\frac{P_{g}}{A_{c}}\left(q_{\mathrm{rad}}^{\prime \prime}-q_{\mathrm{conv}}^{\prime \prime}-q_{\mathrm{dcht}}^{\prime \prime}\right)
$$

This equation is solved for the change in grid temperature from the previous time step. The specific heat, $C_{p, g}$, and the density, $\rho_{g}$, are the material properties of the grid. The wetted perimeter and cross-sectional area of the grid strap are characterized by $P_{g}$ and $A_{c}$, respectively. The cross-sectional area of the grid strap is defined in terms of the grid strap thickness, $t_{g}$, by the following expression:

$$
A_{c}=\frac{1}{2} t_{g} P_{g}
$$

Equation 6.182 can be reduced:

$$
\Delta T_{g}=\Delta t \frac{q_{\mathrm{rad}}^{\prime \prime}-q_{\mathrm{conv}}^{\prime \prime}-q_{\mathrm{dcht}}^{\prime \prime}}{\frac{1}{2} t_{g} \rho_{\text {grid }} C_{p, \text { grid }}}
$$

This leaves the three heat flux terms to be calculated.

Radiative Heat Flux For simplicity, it is assumed that the rod is completely enclosed by the grid, as shown in Figure 6.17. The rod diameter is given by $D_{1}$ and the grid diameter (physically, the grid pitch) is given by $D_{2}$.

For determining the radiant heat flux to the grid, the model of Figure 6.17 is broken up into a representative heat transfer resistance network, which includes heat transfer from rod to vapor, rod to grid, and vapor to grid. It is shown in Figure 6.18.

The resistance network has terms $-B_{1}$ and $B_{2}$-representing the black body radiosity of the rod and grid respectively. The heat flux to the rod can be expressed as:

$$
q_{1}^{\prime \prime}=\frac{B_{1}-\sigma T_{1}^{4}}{A_{1} R_{11}}
$$




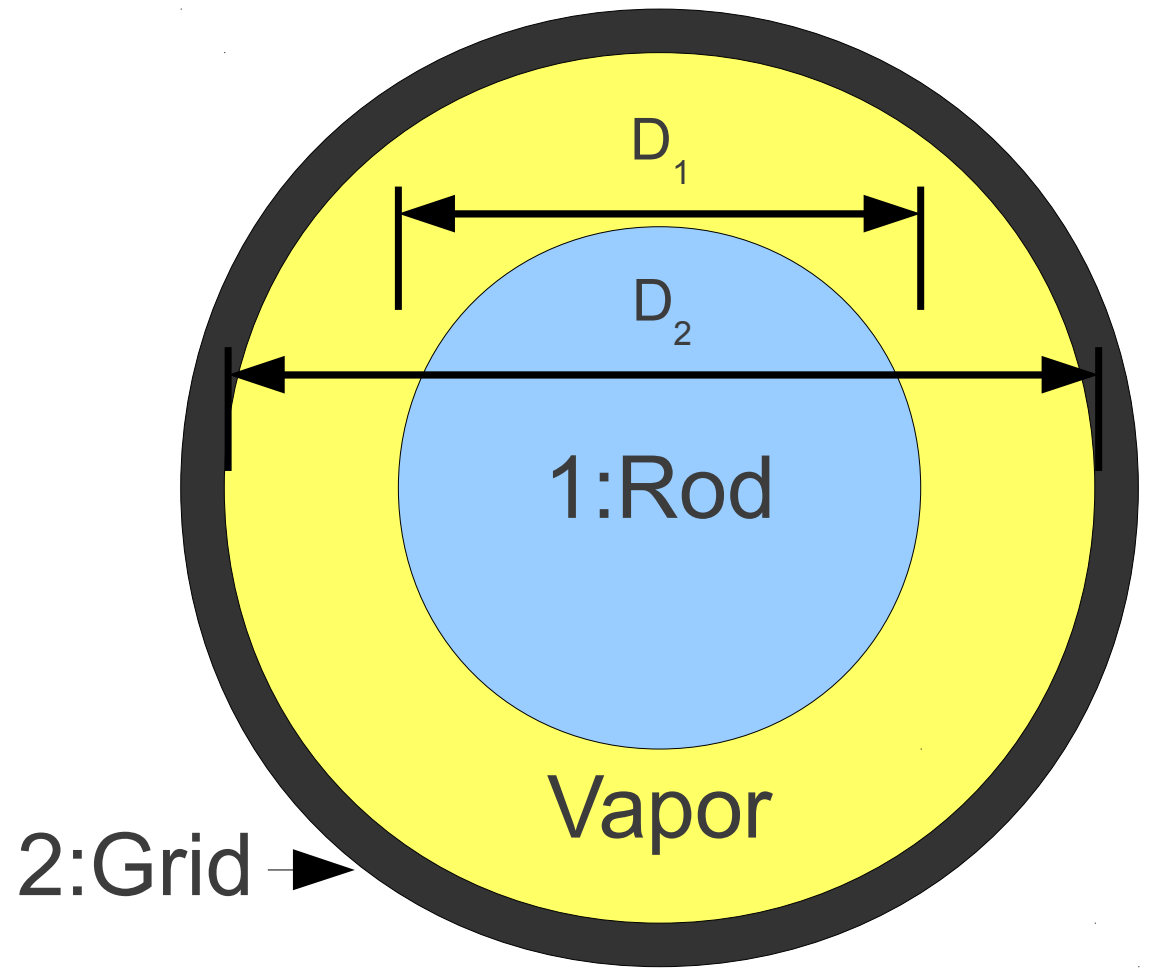

Figure 6.17: Model of rod and grid for calculating radiative heat transfer to grid

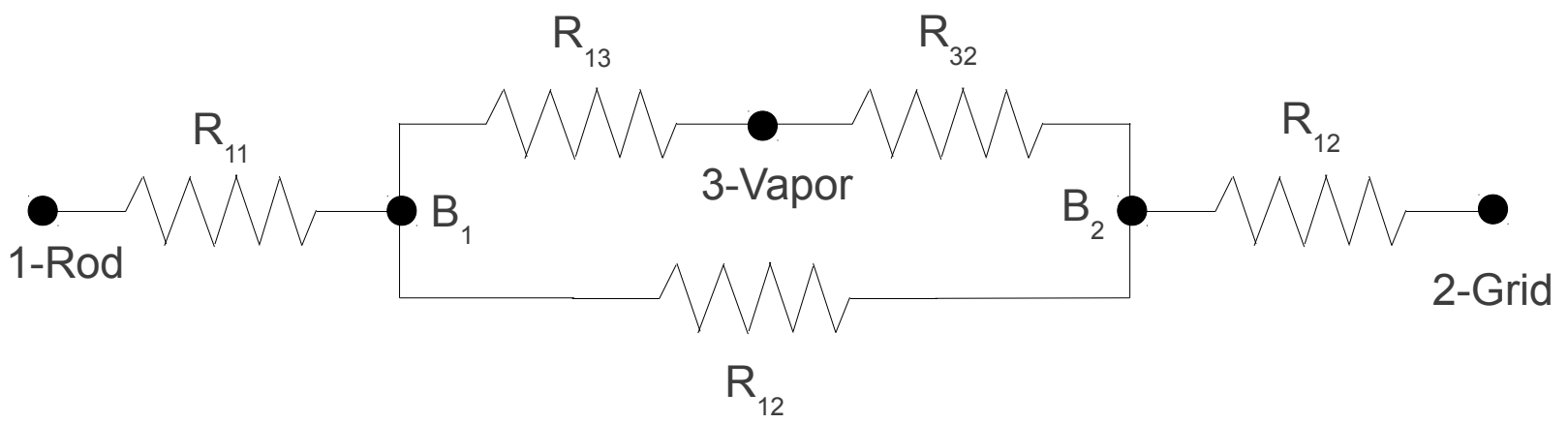

Figure 6.18: Grid-to-rod radiative heat transfer resistance network 
The Stefan-Boltzmann constant - $\sigma$-is $0.1714 \cdot 10^{-8} \mathrm{BTU} / \mathrm{hr}-\mathrm{ft}^{2} \cdot \mathrm{R}^{4}$. The surface area of the rod is given by $A_{1}$. The radiative heat transfer to the rod is also expressed in terms of the radiative heat transfer contributions from the grid and vapor.

$$
q_{1}^{\prime \prime}=\frac{B_{1}-B_{2}}{A_{2} R_{21}}+\frac{\sigma T_{3}^{4}-B_{2}}{A_{2} R_{23}}
$$

The radiative heat transfer to the grid can similarly be defined as:

$$
q_{2}^{\prime \prime}=\frac{B_{2}-\sigma T_{2}^{4}}{A_{2} R_{22}}=\frac{B_{1}-B_{2}}{A_{2} R_{21}}+\frac{\sigma T_{3}^{4}-B_{2}}{A_{2} R_{23}}
$$

To solve for the radiative heat transfer to the rod and the grid, it is necessary to use Equations 6.185 and 6.186 to solve for the black body radiosity of the grid in terms of black body radiosity of the rod. This expression can then be substituted into Equation 6.187 in order to solve for the rod black body radiosity. The grid spacer black body radiosity is first obtained by manipulating Equations 6.185 and 6.186.

$$
B_{2}=\left[\frac{B_{1}-\sigma T_{1}^{4}}{A_{1} R_{11}}-\frac{\sigma T_{3}^{4}-B_{1}}{A_{1} R_{13}}\right] A_{1} R_{12}+B_{1}
$$

This expression is substituted into Equation 6.187, which is solved for $B_{1}$. This leads to a sizeable expression that is reduced using four coefficients.

$$
B_{1}=\frac{C_{1} \sigma T_{1}^{4}+C_{2} \sigma T_{2}^{4}+C_{3} \sigma T_{3}^{4}}{C_{4}}
$$

The coefficients are:

$$
\begin{aligned}
& C_{1}=D\left(A_{1} R_{12}\right)\left(A_{1} R_{13}\right) \\
& C_{2}=\left(A_{1} R_{11}\right)\left(A_{1} R_{13}\right)\left(A_{2} R_{21}\right)\left(A_{2} R_{23}\right) \\
& C_{3}=\left(A_{1} R_{11}\right)\left[\left(A_{1} R_{13}\right)\left(A_{2} R_{21}\right)\left(A_{2} R_{22}\right)+\left(A_{1} R_{12}\right) D\right] \\
& C_{4}=\left(A_{1} R_{11}\right)\left(A_{1} R_{13}\right)\left(A_{2} R_{21}\right)\left[\left(A_{2} R_{23}+A_{2} R_{22}\right)\right]+\left(A_{1} R_{12}\right)\left[\left(A_{1} R_{13}\right) D+\left(A_{1} R_{11}\right) D\right] \\
& D=\left(A_{2} R_{21}\right)\left(A_{2} R_{23}\right)+\left(A_{2} R_{22}\right)\left(A_{2} R_{23}+A_{2} R_{21}\right)
\end{aligned}
$$

The rod black body radiosity is a function of grid, rod, and vapor temperature, surface area, and radiative heat transfer resistance and can be solved. This expression can be substituted into Equation 6.188 to define the grid black body radiosity as well. The black body radiosities can then be substituted back into Equations 6.185 and 6.187 to obtain the rod and grid radiative heat fluxes, respectively. But first, it is necessary to define the radiative heat transfer resistances, which are functions of surface emissivity and surface area. 


$$
\begin{aligned}
& R_{11}=\frac{1-\epsilon_{1}}{A_{1} \epsilon_{1}} \\
& R_{12}=\frac{1}{A_{1} F_{12}\left(1-\epsilon_{3}\right)} \\
& R_{22}=\frac{1-\epsilon_{2}}{A_{2} \epsilon_{2}} \\
& R_{21}=R_{12} \\
& R_{13}=\frac{1}{A_{1} \epsilon_{3}} \\
& R_{23}=\frac{1}{A_{2} \epsilon_{3}}
\end{aligned}
$$

The resistances are in units of $\mathrm{ft}^{-2}$. The emissivity of the rod and grid $-\epsilon_{1}$ and $\epsilon_{2}$-are both assumed to be 0.9. The emissivity of the vapor is calculated using the Plank mean absorption coefficient as reported by Sparrow $[100]$.

$$
\epsilon_{3}=1.0-\exp \left[-A_{v} P L_{m}\right]
$$

The pressure is represented by $P$ and the mean beam length by $L_{m}$. The mean beam length is a constant value of $6.9083 \cdot 10^{-3} \mathrm{ft}$. The vapor absorption coefficient (units of $(\mathrm{psi}-\mathrm{ft})^{-1}$ ) is defined using the Plank mean absorption coefficient:

$$
A_{v}=2.146 \exp \left[-0.344523221-\left(2.96092004 \cdot 10^{-3}-0.444073925 \cdot 10^{-6} T_{v}\right) T_{v}\right]
$$

Also necessary for the calculation of the heat transfer resistance is the rod-to-grid view factor $-F_{12}-$ which is the fraction of the radiation leaving the rod that is intercepted by the grid. Due to the modeling of the grid shown in Figure 6.17, the rod-to-grid view factor will be 1. Other view factors are provided below:

$$
\begin{aligned}
& F_{11}=0 \\
& F_{12}=1 \\
& F_{21}=\frac{D_{1}}{D_{2}} \\
& F_{22}=1-\frac{D_{1}}{D_{2}}
\end{aligned}
$$

Equation 6.187 is solved to determine radiative heat transfer to the grid, which is later substituted into Equation 6.184 as the first heat transfer term. 
Grid Convective Heat Transfer The heat transfer from the grid is defined as follows:

$$
q_{\text {conv }}^{\prime \prime}=h_{\text {rod }}\left(\tilde{T}_{g}-T_{v}\right)
$$

The heat transfer coefficient used is the same heat transfer coefficient that was calculated for the rod-tovapor in the HEAT subroutine. The grid temperature in the dry region, $T_{g}$, and the vapor temperature, $T_{v}$, are used. The grid is located at the top of the continuity mesh cell, so the upper-most fine-mesh node heat transfer coefficient is used. The old time step grid temperature is used explicitly.

Droplet Impingement Heat Transfer There is a final heat transfer term which results from droplets impacting the dry portion of the grid. As the droplets vaporize, they remove heat from the grid. The heat transfer due to de-entrainment is assumed equal to the de-entrainment rate times the latent heat of vaporization and an efficiency factor that defines the number of droplets that evaporate.

$$
q_{\mathrm{dcht}}^{\prime \prime}=S_{d e} h_{\mathrm{fg}} \eta
$$

Droplets are considered to impact the grid due to turbulent interchange causing lateral migration of grids. Therefore, a lateral deposition rate must be calculated. This is done using a coefficient times the droplet axial mass flux.

$$
S_{d e}=C_{d e} G_{e}
$$

The deposition coefficient is defined by Paik[101].

$$
C_{d e}=0.102 \sqrt{\frac{\mu_{v} f}{D_{h} \sigma \rho_{l}}}
$$

The droplet evaporation factor, $\eta$, is defined using the old time grid temperature and liquid saturation temperature (absolute values in units of Rankine).

$$
\eta=\exp \left[1-\left(\frac{T_{g}}{T_{f}}\right)^{2}\right]
$$

This final heat flux calculated by Equation 6.196 is substituted into the dry region heat balance-Equation 6.184 - and the dry region grid temperature is calculated.

\subsubsection{Wet Region Heat Balance}

The wet region heat fluxes are calculated similar to the dry region, but for a different purpose than determining the grid temperature. The grid temperature is assumed to be equal to the saturation temperature 
in the wet region since the grid is quenched. The purpose of the heat flux calculations is to determine the evaporation rate of the film that quenches the grid. This information is compared to the droplet deposition rate to determine whether the quench front will advance or recede. Droplet impingement in the wet region does not cause heat transfer from the grid, but instead causes the quenching film to grow.

The heat balance is calculated using the radiative and convective components:

$$
q=\left(q_{\mathrm{conv}, \mathrm{w}}^{\prime \prime}+q_{\mathrm{radw}}^{\prime \prime}\right) P_{w} L_{\mathrm{qf}}
$$

The product of the wetted perimeter, $P_{w}$, and the length of the quenched portion of the grid, $L_{\mathrm{qf}}$, gives the surface area of the wetted region of the grid.

Radiative Heat Transfer The wet region radiative heat transfer from the fuel rods is similar to the radiative heat transfer calculation done in the dry region. The black body radiosity of the rod is first repeated:

$$
B_{1}=\frac{C_{1} \sigma T_{1}^{4}+C_{2} \sigma T_{2}^{4}+C_{3} \sigma T_{3}^{4}}{C_{4}}
$$

Since the grid temperature is equal to the saturation temperature, the black body radiosity is modified as follows:

$$
B_{1, w}=\frac{C_{1} \sigma T_{1}^{4}+C_{2} \sigma T_{f}^{4}+C_{3} \sigma T_{3}^{4}}{C_{4}}
$$

The emissivity of the liquid film is assumed equal to the grid emissivity calculated for the dry region. The radiative heat transfer is calculated as follows:

$$
q_{\mathrm{radw}}^{\prime \prime}=\frac{B_{2}-\sigma T_{f}^{4}}{A_{2} R_{22}}
$$

Convective Heat Transfer The convective heat flux is defined as:

$$
q_{\mathrm{conv}, \mathrm{w}}^{\prime \prime}=h_{w}\left(T_{v}-T_{f}\right)
$$

The convective heat transfer coefficient is calculated in INTFR. The Nusselt number is calculated as follows:

$$
\begin{aligned}
& N u=\max \left[10,0.0797 \operatorname{Re}_{v}^{0.6774} \operatorname{Pr}_{v}^{0.333}\right], \quad \text { if } R e_{v}<=25,200 \\
& N u=0.023 \operatorname{Re}_{v}^{0.8} \operatorname{Pr}_{v}^{0.4}, \quad \text { if } R e_{v}>25,200
\end{aligned}
$$


The convective heat transfer coefficient is calculated from the film conductivity, the wetted grid surface area, and a two-phase enhancement factor.

$$
h_{w}=T \frac{k_{l}}{D_{h}} N u A
$$

The two phase enhancement factor is calculated as follows:

$$
T=\min \left[10, \sqrt{1+\frac{0.75 \alpha_{e} D_{h} C_{d}}{D_{d} f} \min \left(1, \frac{\left(u_{v}-u_{e}\right)}{u_{v}^{2}}\right.}\right]
$$

$C_{d}$ is the droplet drag coefficient and $D_{d}$ is the droplet diameter. The friction coefficient is calculated as follows:

$$
f=\frac{0.158}{\max \left[100, R e_{v}\right] .25}
$$

\subsubsection{Grid Quench Front Model}

The grid quench front model tracks the fraction of the grid that is wetted. The wet region heat balance from the previous section is used to determine whether the quench front grows or recedes. This is done by comparing the evaporation rate (due to radiative and convective heat transfer into the grid film) with the droplet deposition heat transfer rate. The droplet deposition rate is the droplet flow rate times the blockage area times a de-entrainment efficiency factor.

$$
\dot{m}_{d e}=G_{e}\left(A_{g}+A_{s}\right) \eta
$$

The droplet mass flux is given by $G_{e}$ and the grid and spring cross-sectional areas - $A_{g}$ and $A_{s}$ - are summed to obtain the total grid blockage area. The final term, $\eta$, is used to account for the fact that all of the droplets deposited on the grid will not be available for film advancement. This is due to the fact that some of the film will be blown off of the grid due to sputtering at the quench front. This factor is similar to the de-entrainment factor used in the dry grid region for droplet impingement heat transfer.

$$
\eta=\max \left[0.025, \exp \left(1-\left(\frac{T_{g}}{T_{f}}\right)^{2}\right)\right]
$$

The heat transfer due to droplet deposition is:

$$
q_{\mathrm{dcht}}=\dot{m}_{\mathrm{de}} h_{\mathrm{fg}}
$$


This value is compared to the evaporation heat transfer calculated for the wet region in the previous section. If the difference between the de-entrainment heat transfer and evaporation heat transfer is positive, there is more droplet deposition than what is evaporating away. Conversely, a negative value will lead to more evaporation than deposition and the quench front will recede. The quench front velocity is calculated to determine how the quench front progresses on the grid. For a net deposition of droplets in the film (advancement of the quench front), the quench front velocity is calculated using the two-region analytical conduction solution of Yamanouchi[102]. The quench front velocity is limited by conduction from the dry to wet regions of the grid.

$$
V_{q}=\left[\frac{\rho_{g} C_{p, g}}{2}\left(\frac{\delta}{h_{w} k_{g}}\right)^{1 / 2}\left\{\left(1+2 \frac{T_{g}-T_{w}}{T_{w}-T_{f}}\right)^{2}-1\right\}^{1 / 2}\right]^{-1}
$$

The terms are:

$$
\begin{array}{ll}
\rho_{g} & \text { Grid density } \\
C_{p, g} & \text { Grid specific heat } \\
k_{g} & \text { Grid conductivity } \\
\delta & \text { Grid strap half thickness } \\
h_{w} & \text { Heat transfer coefficient of the wet region } \\
T_{w} & \text { Minimum film boiling temperature }\left(T_{f}+234^{\circ} \mathrm{F}\right)
\end{array}
$$

This velocity is limited using the wet region heat transfer. The heat transfer at the quench front should be at its maximum. The heat transfer coefficient is limited using the critical heat flux defined by Zuber [72], given by Equation 6.63 .

$$
h_{w}=\frac{q_{\mathrm{chf}}^{\prime \prime}}{T_{w}-T_{f}}
$$

A second limit is placed on the quench front velocity to account for the fact that a limited amount of water is available for advancing the quench front. The net de-entrainment rate is:

$$
\dot{m}_{\mathrm{QF}, \mathrm{net}}=\dot{m}_{d e}-\frac{\left(q_{\mathrm{radw}}^{\prime \prime}+q_{\mathrm{conv}}^{\prime \prime}\right) P_{g} L_{g}}{h_{\mathrm{fg}}}
$$

The energy removed at the quench front cannot exceed the liquid availability times the latent heat of vaporization. The energy removed by quenching is a function of the velocity of the quench front. The limit can be imposed as follows:

$$
\dot{m}_{\mathrm{QF}, \text { net }} h_{\mathrm{fg}}>=\left(\rho C_{p} A_{x}\right)_{g} V_{q f}\left(T_{g}-T_{f}\right)
$$

This limits the quench front velocity to: 


$$
V_{q f}<=\frac{\dot{m}_{\mathrm{QF}, \text { net }} h_{\mathrm{fg}}}{\left(\rho C_{p} A_{x}\right)_{g}\left(T_{g}-T_{f}\right)}
$$

If this limit is substituted into Equation 6.211, the Yamanouchi quench front velocity equation, the maximum value of the wet region heat transfer coefficient can be determined.

$$
h_{w}<=\left(\frac{\delta}{4 k_{g}}\right)\left(\frac{\dot{m}_{\mathrm{QF}, \text { net }} h_{\mathrm{fg}}}{P_{g} \delta\left(T_{g}-T_{f}\right)}\right)^{2}\left[\left(\frac{2\left(T_{g}-T_{w}\right)}{T_{w}-T_{f}}+1\right)^{2}-1\right]
$$

The wet region heat transfer is limited to the values calculated by Equations 6.212 and 6.216. The wet region heat transfer is further limited to a maximum value of $1 \cdot 10^{-6} \mathrm{BTU} / \mathrm{hr} \cdot \mathrm{ft}^{2} \mathrm{~F}$.

If the net deposition rate is a negative value, and the quench front is receding, the quench front velocity is calculated in a more straightforward manner.

$$
V_{q f}=\frac{\dot{m}_{\mathrm{QF}, \mathrm{net}} h_{\mathrm{fg}}}{\left(\rho C_{p} A_{x}\right)_{g}\left(T_{g}-T_{f}\right)}
$$

The quench front location is calculated at each time step using the time step size and velocity of the quench front.

$$
L_{\mathrm{qf}}=\tilde{L}_{\mathrm{qf}}+V_{q f} \Delta t
$$




\section{Spacer Grid Models}

\subsection{Grid Heat Transfer Enhancement}

Spacer grids are used in nuclear reactors to maintain the rod-bundle geometry through the normal operation of the reactor. The presence of these grids is known to impact the flow due to their non-negligible thickness that causes acceleration/deceleration of the flow, creation of secondary flows, and disruption of the boundary layer. One of the most important effects of the grid spacers to be able to model is the effect that these flow redistributions have on rod-to-fluid heat transfer.

Yao, Hochreiter, and Leech [85] have studied this effect for egg-crate and honeycomb-style spacer grids and proposed correlations for the effect of the grids on the Nusselt number. Their work focused on heat transfer effects both upstream and downstream of the spacer for both mixing and non-mixing vane grid styles. Noting the analogy between pressure-drop effects and heat-transfer effects, and using experimental heat-transfer enhancement data from a collection of grid styles and different flow conditions, the authors have proposed the following exponentially decaying correlation for heat-transfer enhancement effects experienced downstream of the grid:

$$
\frac{N u}{N u_{0}}=1+5.55 \epsilon^{2} \exp \left[-0.13 \frac{x}{D}\right]
$$

The heat transfer enhancement is a function of $\epsilon$, which is the blockage ratio of the grid $\left(A_{\text {blocked }} / A_{\text {bare }}\right)$ as well as the downstream, non-dimensional distance from the end of the spacer grid $(x / D)$. The nondimensional distance from the end of the spacer grid is calculated by dividing the absolute distance from the end of the grid by the hydraulic diameter of the test section (bare region). This correlation applies to single-phase flow (liquid or vapor) for non-mixing vane grid designs.

The authors have additionally suggested a means for capturing the swirling effect of vanes on the flow when mixing-vane grid styles are to be modeled. The presence of mixing vanes has been observed to cause swirling of the fluid, leading to increased local velocities and fluid mixing which, in turn, leads to increased heat transfer. The authors proposed a simple model taking into account the fraction of the projected area of the vanes as seen by the flow, $A$, the angle of the vanes, $\Phi$, and again, the downstream distance from the end of the grid, $x / D$. 


$$
\frac{N u}{N u_{0}}=\left[1+A^{2} \tan ^{2}(\Phi) \exp [-0.034 x / D]\right]^{0.4}
$$

Since data from real mixing vane grids were not available, the authors used in-tube gas-flow experimental data to develop the correlation. Because of this, the mixing-vane component of the correlation is not typically utilized.

Regardless, for maximum user-flexibility, the model has been implemented into CTF in its full form, but with the coefficients replaced with user-input variables, $a, b, c$, and $d$, as shown in Equation 7.3.

$$
\frac{N u}{N u_{0}}=\left[1+a \epsilon^{2} \exp \left[b \frac{x}{D}\right]\right]\left[1+A^{2} \tan ^{2}(\Phi) \exp \left[c \frac{x}{D}\right]\right]^{d}
$$

In this way, the user is free to set $d$ to 0.0 , thus disabling the mixing-vane component of the correlation.

The authors also determined the upstream heat transfer effects of the grid to be linearly dependent on distance from the grid; however, that contribution of the grid to heat transfer has not been included in CTF. In activating the model, the user has the option to turn the model on for only single-phase vapor, or both single-phase liquid and single phase-vapor. If used for both phases, and two-phase conditions persist, an equal heat transfer enhancement factor will be multiplied by the rod heat transfer coefficient of each of the phases. 


\section{Fluid Properties}

CTF is capable of calculating thermal conductivity, specific heat, viscosity, surface tension, and enthalpy for water. Three conditions of water may exist that require separate attention: subcooled liquid, superheated vapor, and saturated mixtures of both phases. These cases are separately covered in the following three sections.

\subsection{Original Water Properties}

CTF's original steam tables, utilized when IPROPS $=0$, are a mixture of several sources. They are the default option when running CTF. Their complete origins are somewhat unknown, but most property evaluations have been traced to existing documentation. This uncertainty prompted the inclusion of newer, more documented steam tables which could be verified by sources.

\subsubsection{Saturated Liquid and Vapor}

Saturated liquid and vapor enthalpies are calculated as a function of pressure based on expressions developed for EPRI [103]. Other state properties, including density, saturation temperature, thermal conductivity, viscosity, specific heat, and surface tension are interpolated from lookup tables that are indexed by the saturated liquid enthalpy. Both the correlations used for determining saturation enthalpies as well as the other state property lookup tables provide close agreement with the ASME Steam Tables [104] and the NBS/NRC Steam Tables [105].

Saturation enthalpies are calculated in BTU/lbm as a function of pressure in psia. Liquid and vapor saturation enthalpies are calculated in the SAT subroutine. The polynomial expression for saturated liquid enthalpy is:

$$
\begin{aligned}
& H_{f}\left(P_{\text {sat }}\right)=\Sigma_{n=1}^{9} A_{n}\left[\ln \left(P_{\text {sat }}\right)\right]^{n-1}, \text { for } P_{\text {sat }}<2529.9 \text { psia } \\
& H_{f}\left(P_{\text {sat }}\right)=\Sigma_{n=1}^{9} A_{n}\left[\left(3208.2-P_{\text {sat }}\right)^{0.41}\right]^{n-1}, \quad \text { for } 2529.9 \mathrm{psia}<=P_{\text {sat }}<3208.0 \mathrm{psia}
\end{aligned}
$$

The constants for the polynomial expansion, $A_{n}$, are given in Table 2. 
Table 2: Polynomial expansion constant for the saturated liquid enthalpy correlation

\begin{tabular}{|l|r|r|r|}
\hline & \multicolumn{3}{|c|}{ Pressure } \\
$A_{n}$ & $0.1<=P<898.7$ & $898.7<=P<2529.9$ & $2529.9<=P<3208$ \\
\hline & & & \\
1 & $0.6970887859 \cdot 10^{+2}$ & $0.8408618802 \cdot 10^{+6}$ & $0.9060030436 \cdot 10^{+3}$ \\
2 & $0.3337529994 \cdot 10^{+2}$ & $0.3637413208 \cdot 10^{+6}$ & $0.1426813520 \cdot 10^{+2}$ \\
3 & $0.2318240735 \cdot 10^{+1}$ & $-0.4634506669 \cdot 10^{+6}$ & $0.1522233257 \cdot 10^{+1}$ \\
4 & $0.1840599513 \cdot 10^{+0}$ & $0.1130306339 \cdot 10^{+6}$ & $-0.6973992961 \cdot 10^{+0}$ \\
5 & $-0.5245502294 \cdot 10^{-2}$ & $-0.4350217298 \cdot 10^{+3}$ & $0.1743091663 \cdot 10^{+0}$ \\
6 & $0.2878007027 \cdot 10^{-2}$ & $-0.3898988188 \cdot 10^{+4}$ & $-0.2319717696 \cdot 10^{-1}$ \\
7 & $0.1753652324 \cdot 10^{-2}$ & $0.6697399434 \cdot 10^{+3}$ & $0.1694019149 \cdot 10^{-2}$ \\
8 & $-0.4334859620 \cdot 10^{-3}$ & $-0.4730726377 \cdot 10^{+2}$ & $-0.6454771710 \cdot 10^{-4}$ \\
9 & $0.3325699282 \cdot 10^{-4}$ & $0.1265125057 \cdot 10^{+1}$ & $0.1003003098 \cdot 10^{-5}$ \\
\hline
\end{tabular}

The polynomial expression for vapor saturated enthalpy is given as follows:

$$
\begin{aligned}
& H_{g}\left(P_{\text {sat }}\right)=\Sigma_{n=1}^{5} B_{n}\left[\ln \left(P_{\text {sat }}\right)\right]^{n-1}+\Sigma_{n=6}^{9} B_{n}\left[\ln \left(P_{\text {sat }}\right)\right]^{n+3}, \text { for } 0.1 \text { psia }<=P_{\text {sat }}<1467.6 \text { psia } \\
& H_{g}\left(P_{\text {sat }}\right)=\Sigma_{n=1}^{9} B_{n}\left[\ln \left(P_{\text {sat }}\right)\right]^{n-1}, \text { for } 1467.6 \mathrm{psia}<=P_{\text {sat }}<2586.0 \text { psia } \\
& H_{g}\left(P_{\text {sat }}\right)=\Sigma_{n=1}^{9} B_{n}\left[\left(3208.2-P_{\text {sat }}\right)^{0.41}\right]^{n-1}, \text { for } 2586.0 \text { psia }<=P_{\text {sat }}<3208.0 \text { psia }
\end{aligned}
$$

The polynomial expansion constants, $B_{n}$, are provided in Table 3 .

Table 3: Polynomial expansion constant for the saturated vapor enthalpy correlation

\begin{tabular}{|c|r|r|r|}
\hline & \multicolumn{3}{|c|}{ Pressure } \\
$B_{n}$ & $0.1<=P<1467.6$ & $1467.6<=P<2586.0$ & $2586.0<=P<3208$ \\
\hline & $0.1105836875 \cdot 10^{+4}$ & $0.5918671729 \cdot 10^{+6}$ & $0.9059978254 \cdot 10^{+3}$ \\
2 & $0.1436943768 \cdot 10^{+2}$ & $-0.2559433320 \cdot 10^{+6}$ & $0.5561957539 \cdot 10^{+1}$ \\
3 & $0.8018288621 \cdot 10^{+0}$ & $0.3032474387 \cdot 10^{+5}$ & $0.3434189609 \cdot 10^{+1}$ \\
4 & $0.1617232913 \cdot 10^{-1}$ & $0.4109051958 \cdot 10^{+1}$ & $-0.6406390628 \cdot 10^{+0}$ \\
5 & $-0.1501147505 \cdot 10^{-2}$ & $0.3475066877 \cdot 10^{+0}$ & $0.5918579484 \cdot 10^{-1}$ \\
6 & $-0.1237675562 \cdot 10^{-4}$ & $-0.3026047262 \cdot 10^{+0}$ & $-0.2725378570 \cdot 10^{-2}$ \\
7 & $0.3004773304 \cdot 10^{-5}$ & $-0.1022018012 \cdot 10^{+2}$ & $0.5006336938 \cdot 10^{-4}$ \\
8 & $-0.2062390734 \cdot 10^{-6}$ & $0.1591215116 \cdot 10^{+1}$ & 0.0 \\
9 & 0.0 & $-0.6768383759 \cdot 10^{-1}$ & 0.0 \\
\hline
\end{tabular}

The lookup table for other state properties include 90 entries of each property. Property data are shown in Table 4. 


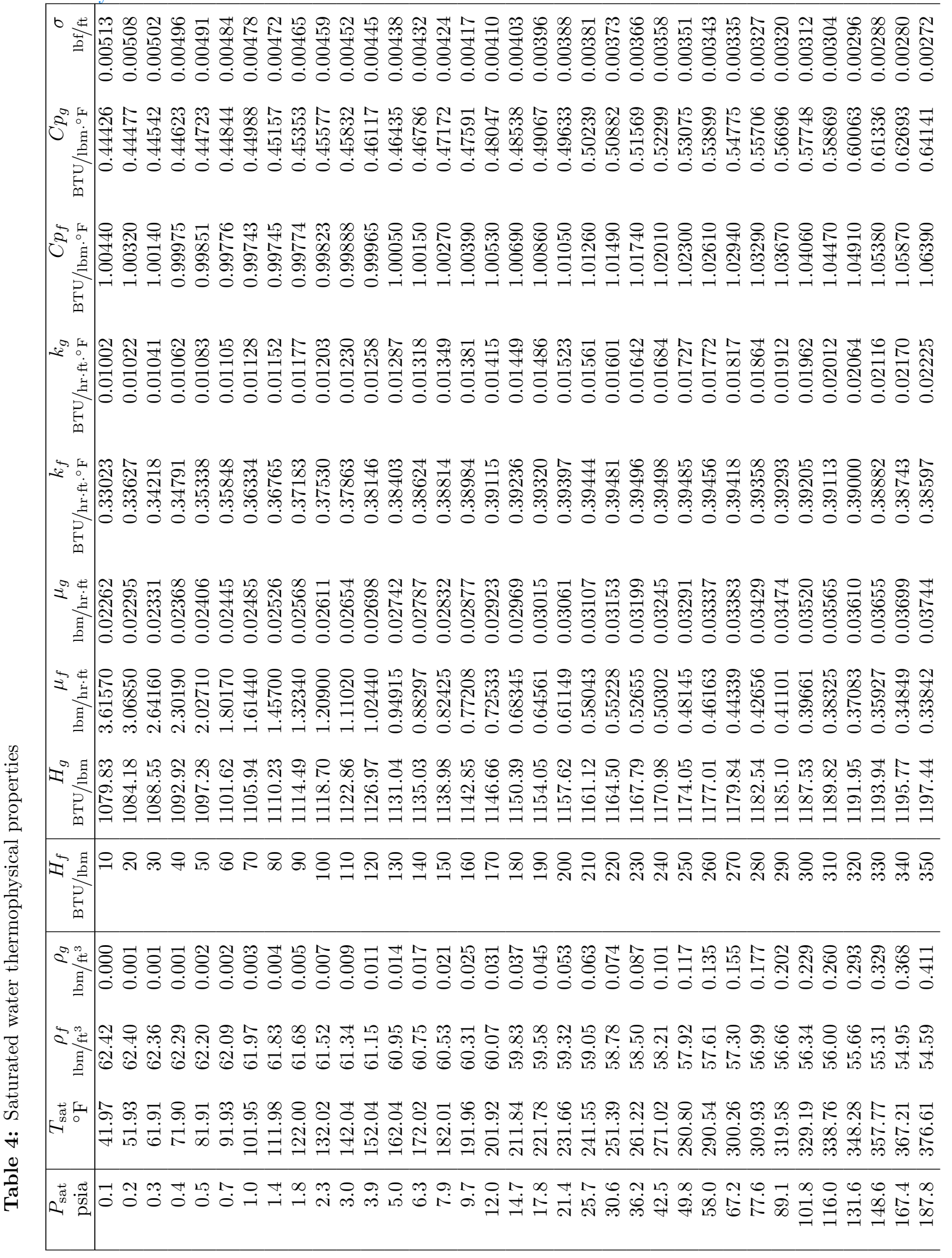




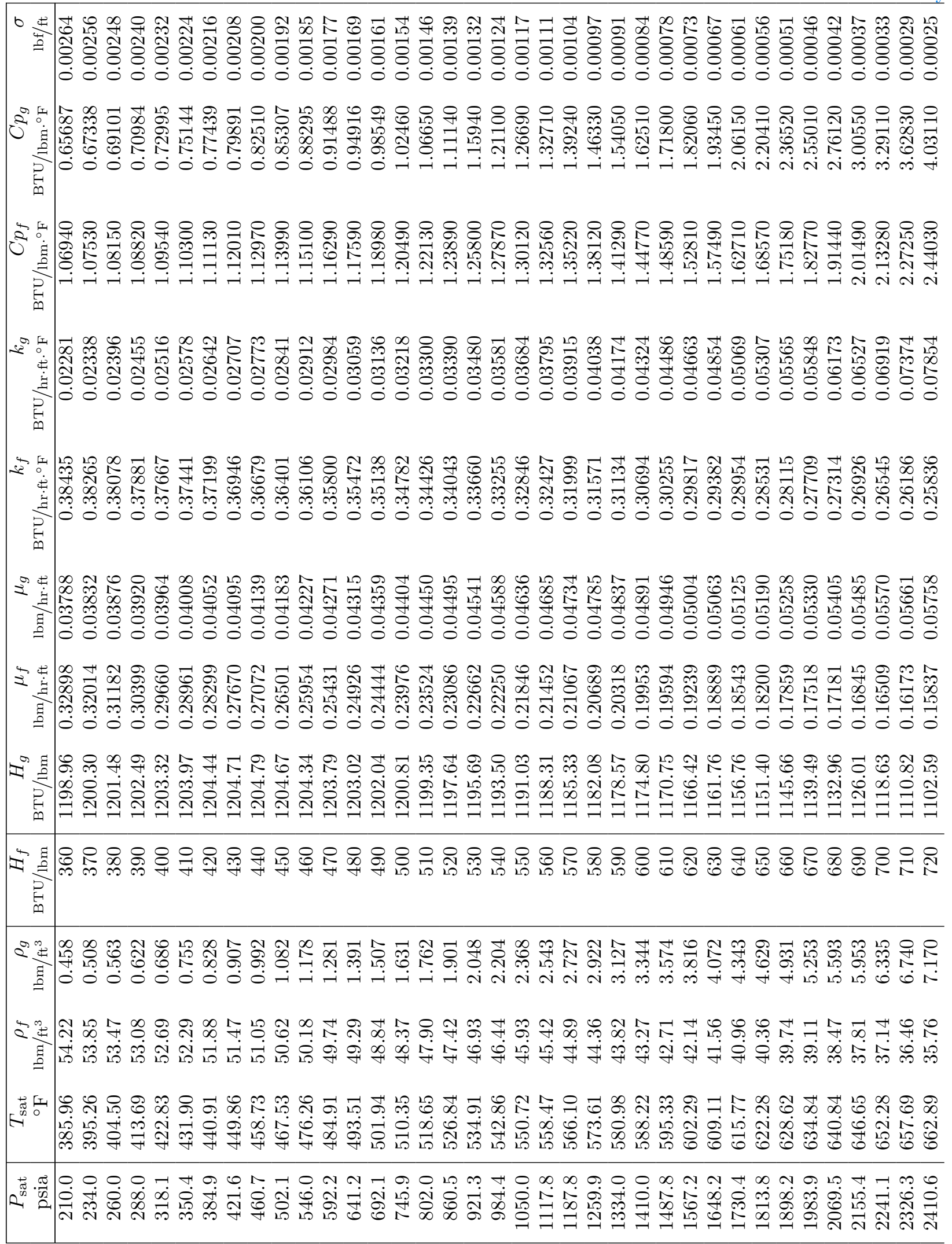




\begin{tabular}{|c|c|}
\hline & \\
\hline b & 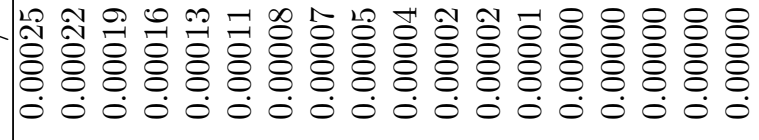 \\
\hline $2^{2}$ & 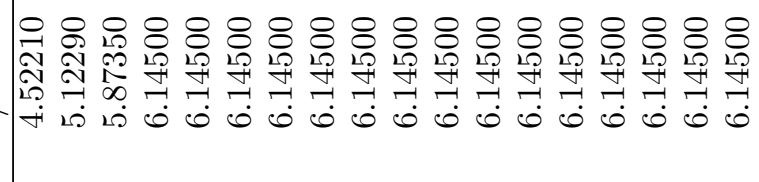 \\
\hline & 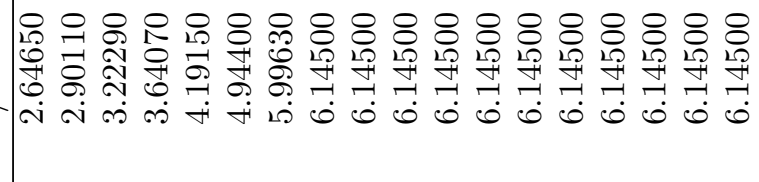 \\
\hline & 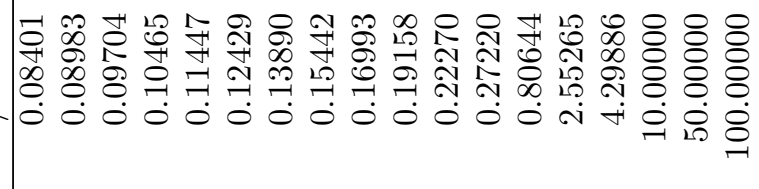 \\
\hline 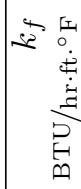 & 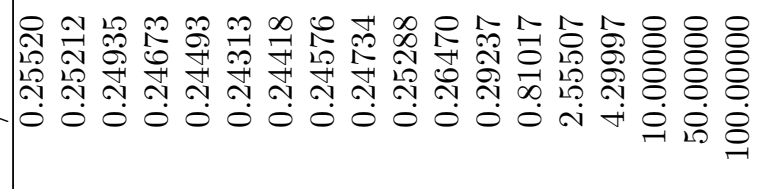 \\
\hline 2 & 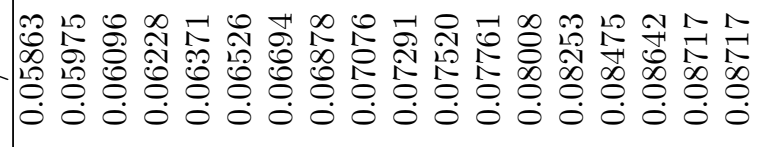 \\
\hline & 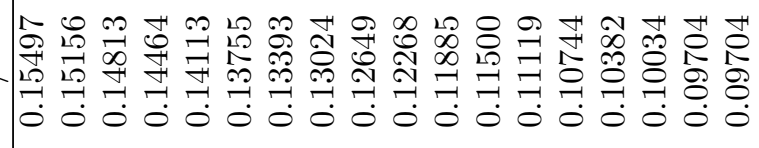 \\
\hline 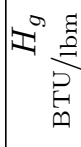 & 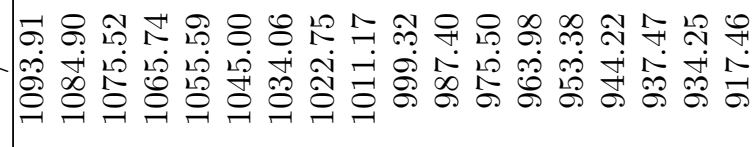 \\
\hline 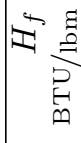 & 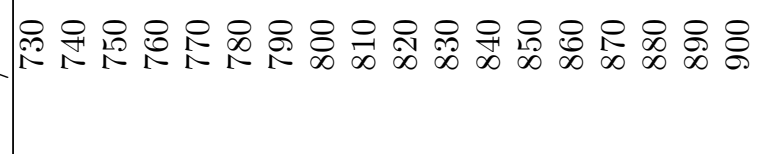 \\
\hline Q⿻日禸 & 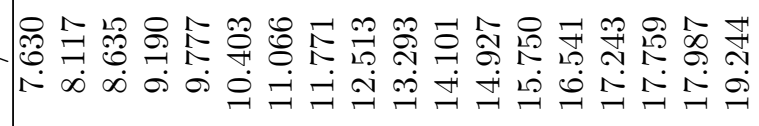 \\
\hline Q & 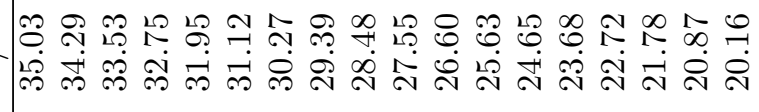 \\
\hline 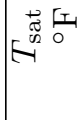 & 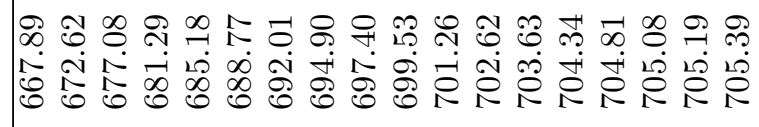 \\
\hline & $\vec{\infty} \ddot{\infty} \vec{\infty} \infty$ \\
\hline
\end{tabular}




\subsubsection{Superheated Vapor}

\subsubsection{Enthalpy}

Enthalpy is calculated in the HGAS subroutine. It is calculated as a function of pressure and temperature using the expression developed by Keenan and Keys [106]. Pressure is provided in atmospheres and Equation 8.3 gives enthalpy in units of $\mathrm{J} / \mathrm{g}$.

$$
H_{v}=0.43\left[0.10129\left(F_{0} P+\frac{F_{1}}{2} P^{2}+\frac{F_{3}}{4} P^{4}+\frac{F_{12}}{13} P^{13}\right)+F^{1}\right]
$$

The $F$ coefficients are defined as:

$$
F_{k}=\frac{\partial}{\partial \tau}\left(B_{k} \tau\right), \text { for } k=0,1,3,12
$$

where: $\tau=$ the inverse of the temperature $\left({ }^{\circ} \mathrm{K}\right), 1 / T$

$B_{k}=$ coefficients

The $B_{k}$ coefficients are defined as:

$$
\begin{aligned}
B_{0} & =1.89-2641.62 \tau \cdot 10^{80870 \tau^{2}} \\
B_{1} & =B_{0}^{2}\left(82.546 \tau^{2}-1.6246 \cdot 10^{5} \tau^{3}\right) \\
B_{3} & =B_{0}^{4}\left(0.21828 \tau^{3}-1.2697 \cdot 10^{5} \tau^{5}\right) \\
B_{12} & =-B_{0}^{13}\left(3.635 \cdot 10^{-4} \tau^{12}-6.768 \cdot 10^{64} \tau^{36}\right)
\end{aligned}
$$

The $F^{1}$ term of Equation 8.3 is defined as:

$$
F^{1}=2502.36+\int_{273.16}^{T}\left(1.472+0.00075566 T+\frac{47.8365}{T}\right) d T
$$

\subsubsection{Density}

Superheated vapor density is calculated in HGAS. The specific volume is first calculated.

$$
\nu=4.55504 \frac{T}{P}+B_{0}+\left[\left(B_{0} \tau\right)^{2} G_{1} P+\left(B_{0} \tau\right)^{4} G_{2} P^{3}-\left(B_{0} \tau\right)^{13} G_{3} P^{12}\right] T
$$

where: $T=$ temperature $\left({ }^{\circ} \mathrm{K}\right)$

$P=$ pressure $(\mathrm{atm})$

$B_{0}=$ coefficient is defined as it was for enthalpy in Equation 8.5

$\tau=$ the inverse of the temperature $\left({ }^{\circ} \mathrm{K}\right), 1 / T$ 
The $G$ terms are defined as follows:

$$
\begin{aligned}
& G_{1}=82.546 \tau-1.6246 \cdot 10^{5} \tau^{2} \\
& G_{2}=0.21828-1.2697 \cdot 10^{5} \tau^{2} \\
& G_{3}=3.635 \cdot 10^{-4}-6.768 \cdot 10^{64} \tau^{24}
\end{aligned}
$$

The density is calculated as follows:

$$
\rho=\frac{62.428}{\nu}
$$

where: $\nu=$ specific volume $\left(\mathrm{cm}^{\wedge} 3 / \mathrm{g}\right)$

$\rho=\operatorname{density}\left(\mathrm{lbm} / \mathrm{ft}^{\wedge} 3\right)$

\subsubsection{Temperature}

Superheated vapor temperature is calculated in the TGAS subroutine. It is calculated directly from enthalpy and pressure by the method outlined by Wagner and Kruse [107] in the Industrial Standard IAPWS-IF97. One of three possible correlations is used to calculated temperature depending on what region the pressure falls in. The pressure regions are Regions $2 \mathrm{a}, 2 \mathrm{~b}$, and 2c. The division between Regions $2 \mathrm{a}$ and $2 \mathrm{~b}$ is $4 \mathrm{MPa}$. The division between Regions $2 \mathrm{~b}$ and $2 \mathrm{c}$ is calculated by the following equation:

$$
P_{2 \mathrm{~b} \text { threshold }}=10\left(905.843-0.679558 h_{\text {norm }}+0.000128090 h_{\text {norm }}^{2}\right)
$$

where: $h_{\text {norm }}=$ normalized enthalpy to $2000 \mathrm{~kJ} / \mathrm{kg}$ prior to inputting it into the above equation.

For Region 2a, the temperature is then calculated as follows:

$$
T=\Sigma_{i=1}^{34} n_{1}(i) P_{\text {norm }}^{l_{1}(i)}\left(h_{\text {norm }}-2.1\right)^{j_{1}(i)}
$$

where: $h_{\text {norm }}=$ normalized enthalpy to $2000 \mathrm{~kJ} / \mathrm{kg}$ prior to inputting it into the above equation

$P_{\text {norm }}=$ normalized pressure to 10 bar

$n_{1}, l_{1}, j_{1}=$ the coefficients are given in Table 5

The Region $2 \mathrm{~b}$ temperature calculation is performed as follows:

$$
T=\Sigma_{i=1}^{38} n_{2}(i)\left(P_{\text {norm }}-2\right)^{l_{2}(i)}\left(h_{\text {norm }}-2.6\right)^{j_{2}(i)}
$$

where: $n_{2}, l_{2}, j_{2}=$ the coefficients are given in Table 6 
The Region 2c temperature calculation is performed as follows:

$$
T=\Sigma_{i=1}^{23} n_{3}(i)\left(P_{\text {norm }}+25\right)^{l_{3}(i)}\left(h_{\text {norm }}-1.8\right)^{j_{3}(i)}
$$

where: $n_{3}, l_{3}, j_{3}=$ the coefficients are given in Table 7

Table 5: Coefficients for the temperature calculation in Region 2a

\begin{tabular}{|r|r|r|}
\hline$n_{1}$ & $l_{1}$ & $j_{1}$ \\
\hline $0.108990 \cdot 10^{+04}$ & 0 & 0 \\
$0.849517 \cdot 10^{+03}$ & 0 & 1 \\
$-0.107817 \cdot 10^{+03}$ & 0 & 2 \\
$0.331537 \cdot 10^{+02}$ & 0 & 3 \\
$-0.742320 \cdot 10^{+01}$ & 0 & 7 \\
$0.117650 \cdot 10^{+02}$ & 0 & 20 \\
$0.184457 \cdot 10^{+01}$ & 1 & 0 \\
$-0.417927 \cdot 10^{+01}$ & 1 & 1 \\
$0.624782 \cdot 10^{+01}$ & 1 & 2 \\
$-0.173446 \cdot 10^{+02}$ & 1 & 3 \\
$-0.200582 \cdot 10^{+03}$ & 1 & 7 \\
$0.271961 \cdot 10^{+03}$ & 1 & 9 \\
$-0.455113 \cdot 10^{+03}$ & 1 & 11 \\
$0.309197 \cdot 10^{+04}$ & 1 & 18 \\
$0.252266 \cdot 10^{+06}$ & 1 & 44 \\
$-0.617074 \cdot 10^{-02}$ & 2 & 0 \\
$-0.310780 \cdot 10^{+00}$ & 2 & 2 \\
$0.116709 \cdot 10^{+02}$ & 2 & 7 \\
$0.128128 \cdot 10^{+09}$ & 2 & 36 \\
$-0.985549 \cdot 10^{+09}$ & 2 & 38 \\
$0.282245 \cdot 10^{+10}$ & 2 & 40 \\
$-0.359490 \cdot 10^{+10}$ & 2 & 42 \\
$0.172273 \cdot 10^{+10}$ & 2 & 44 \\
$-0.135513 \cdot 10^{+05}$ & 3 & 24 \\
$0.128487 \cdot 10^{+08}$ & 3 & 44 \\
$0.138657 \cdot 10^{+01}$ & 4 & 12 \\
$0.235988 \cdot 10^{+06}$ & 4 & 32 \\
$-0.131052 \cdot 10^{+08}$ & 4 & 44 \\
$0.739998 \cdot 10^{+04}$ & 5 & 32 \\
$-0.551967 \cdot 10^{+06}$ & 5 & 36 \\
$0.371541 \cdot 10^{+07}$ & 5 & 42 \\
$0.191277 \cdot 10^{+05}$ & 6 & 34 \\
$-0.415352 \cdot 10^{+06}$ & 6 & 44 \\
$-0.624599 \cdot 10^{+02}$ & 7 & 28 \\
\hline
\end{tabular}


Table 6: Coefficients for the temperature calculation in Region $2 \mathrm{~b}$

\begin{tabular}{|r|r|r|}
\hline$n_{2}$ & $l_{2}$ & $j_{2}$ \\
\hline & & \\
$0.148950 \cdot 10^{+04}$ & 0 & 0 \\
$0.743078 \cdot 10^{+03}$ & 0 & 1 \\
$-0.977083 \cdot 10^{+02}$ & 0 & 2 \\
$0.247425 \cdot 10^{+01}$ & 0 & 12 \\
$-0.632813 \cdot 10^{+00}$ & 0 & 18 \\
$0.113860 \cdot 10^{+01}$ & 0 & 24 \\
$-0.478119 \cdot 10^{+00}$ & 0 & 28 \\
$0.852081 \cdot 10^{-02}$ & 0 & 40 \\
$0.937471 \cdot 10^{+00}$ & 1 & 0 \\
$0.335931 \cdot 10^{+01}$ & 1 & 2 \\
$0.338094 \cdot 10^{+01}$ & 1 & 6 \\
$0.168445 \cdot 10^{+00}$ & 1 & 12 \\
$0.738757 \cdot 10^{+00}$ & 1 & 18 \\
$-0.471287 \cdot 10^{+00}$ & 1 & 24 \\
$0.150203 \cdot 10^{+00}$ & 1 & 28 \\
$-0.217641 \cdot 10^{-02}$ & 1 & 40 \\
$-0.218108 \cdot 10^{-01}$ & 2 & 2 \\
$-0.108298 \cdot 10^{+00}$ & 2 & 8 \\
$-0.463333 \cdot 10^{-01}$ & 2 & 18 \\
$0.712804 \cdot 10^{-04}$ & 2 & 40 \\
$0.110328 \cdot 10^{-03}$ & 3 & 1 \\
$0.189552 \cdot 10^{-03}$ & 3 & 2 \\
$0.308915 \cdot 10^{-02}$ & 3 & 12 \\
$0.135555 \cdot 10^{-02}$ & 3 & 24 \\
$0.286402 \cdot 10^{-06}$ & 4 & 2 \\
$-0.107799 \cdot 10^{-04}$ & 4 & 12 \\
$-0.764627 \cdot 10^{-04}$ & 4 & 18 \\
$0.140524 \cdot 10^{-04}$ & 4 & 24 \\
$-0.310838 \cdot 10^{-04}$ & 4 & 28 \\
$-0.103027 \cdot 10^{-05}$ & 4 & 40 \\
$0.282173 \cdot 10^{-06}$ & 5 & 18 \\
$0.127049 \cdot 10^{-05}$ & 5 & 24 \\
$0.738034 \cdot 10^{-07}$ & 5 & 40 \\
$-0.110301 \cdot 10^{-07}$ & 6 & 28 \\
$-0.814564 \cdot 10^{-13}$ & 7 & 2 \\
$-0.251805 \cdot 10^{-10}$ & 7 & 28 \\
$-0.175652 \cdot 10^{-17}$ & 9 & 1 \\
$0.869342 \cdot 10^{-14}$ & 9 & 40 \\
\hline
\end{tabular}


Table 7: Coefficients for the temperature calculation in Region 2c

\begin{tabular}{|r|r|r|}
\hline$n_{3}$ & $l_{3}$ & \multicolumn{1}{|c|}{$j_{3}$} \\
\hline$-0.323684 \cdot 10^{+13}$ & -7 & 0 \\
$0.732634 \cdot 10^{+13}$ & -7 & 4 \\
$0.358251 \cdot 10^{+12}$ & -6 & 0 \\
$-0.583401 \cdot 10^{+12}$ & -6 & 2 \\
$-0.107831 \cdot 10^{+11}$ & -5 & 0 \\
$0.208255 \cdot 10^{+11}$ & -5 & 2 \\
$0.610748 \cdot 10^{+06}$ & -2 & 0 \\
$0.859777 \cdot 10^{+06}$ & -2 & 1 \\
$-0.257457 \cdot 10^{+05}$ & -1 & 0 \\
$0.310811 \cdot 10^{+05}$ & -1 & 2 \\
$0.120823 \cdot 10^{+04}$ & 0 & 0 \\
$0.482198 \cdot 10^{+03}$ & 0 & 1 \\
$0.379660 \cdot 10^{+01}$ & 1 & 4 \\
$-0.108430 \cdot 10^{+02}$ & 1 & 8 \\
$-0.453642 \cdot 10^{-01}$ & 2 & 4 \\
$0.145591 \cdot 10^{-12}$ & 6 & 0 \\
$0.112616 \cdot 10^{-11}$ & 6 & 1 \\
$-0.178050 \cdot 10^{-10}$ & 6 & 4 \\
$0.123246 \cdot 10^{-06}$ & 6 & 10 \\
$-0.116069 \cdot 10^{-05}$ & 6 & 12 \\
$0.278464 \cdot 10^{-04}$ & 6 & 16 \\
$-0.592700 \cdot 10^{-03}$ & 6 & 20 \\
$0.129186 \cdot 10^{-02}$ & 6 & 22 \\
\hline
\end{tabular}




\subsubsection{Specific Heat}

The specific heat is calculated as a function of the superheated vapor temperature and pressure. It is calculated by the method outlined by Wagner and Kruse [107] in the Industrial Standard IAPWS-IF97. Pressure is normalized to 10 bar. Temperature is first normalized by $1 / 540 \mathrm{~K}$, as follows:

$$
T_{\text {norm }}=\frac{540}{T}
$$

where: $T=$ temperature $\left({ }^{\circ} \mathrm{K}\right)$

The specific heat is calculated in units of $\mathrm{kJ} / \mathrm{kg}^{\circ} \mathrm{K}$ as follows:

$$
C p=-R_{\mathrm{H}_{2} \mathrm{O}} T_{\text {norm }}^{2}\left(\gamma_{0}+\gamma_{r}\right)
$$

where: $R_{\mathrm{H}_{2} \mathrm{O}}=0.461526$, the specific gas constant for water

$\gamma_{0}$ is defined as follows:

$$
\gamma_{0}=\Sigma_{i=1}^{9} n_{0}(i) j_{0}(i)\left(j_{0}(i)-1\right) T_{\text {norm }}^{j_{0}(i)-2}
$$

where: $n_{0}, j_{0}=$ terms are given in Table 8

$\gamma_{r}$ is defined as follows:

$$
\gamma_{r}=\sum_{i=1}^{43} n r(i) P_{\text {norm }}^{i r(i)} j r(i)(j r(i)-1)\left(T_{\text {norm }}-0.5\right)^{j r(i)-2}
$$

where: $n r, i r, j r=$ coefficient terms are given in Table 9

\subsubsection{Thermal Conductivity}

The thermal conductivity of vapor is calculated in the TRANSP subroutine as a function of temperature and density. It is calculated using equations given in the ASME Steam Tables [104]. The expression for thermal conductivity is given as:

$$
k_{v}=k_{1}+\left[103.51+0.4198 T-2.771 \cdot 10^{-5} T^{2}\right] \rho_{v}+2.1482 \cdot 10^{14} \frac{\rho_{v}^{2}}{T^{4.2}}
$$

with

$$
k_{1}=17.6+5.87 \cdot 10^{-2} T+1.04 \cdot 10^{-4} T^{2}-4.51 \cdot 10^{-8} T^{3}
$$


Table 8: Coefficients for the calculation of $\gamma_{0}$

\begin{tabular}{|r|r|}
\hline$n_{0}$ & $j_{0}$ \\
\hline-9.6927686500217 & 0 \\
10.086655968018 & 1 \\
-0.0056087911283020 & -5 \\
0.071452738081455 & -4 \\
-0.40710498223928 & -3 \\
1.4240819171444 & -2 \\
-4.3839511319450 & -1 \\
-0.28408632460772 & 2 \\
0.021268463753307 & 3 \\
\hline
\end{tabular}

where: $k_{v}, k_{1}=$ thermal conductivity $(\mathrm{W} / \mathrm{m}-\mathrm{K})$

$\rho_{v} \quad=\operatorname{density}\left(\mathrm{g} / \mathrm{cm}^{3}\right)$

$\mathrm{T}=$ temperature $\left({ }^{\circ} \mathrm{C}\right)$

\subsubsection{Viscosity}

Viscosity, like thermal conductivity is determined in the TRANSP subroutine using equations given by the ASME Steam Tables[104].

$$
\mu_{v}=\left\{\begin{array}{l}
\mu_{1}-\rho(1858-5.9 T), \text { if } T<340{ }^{\circ} \mathrm{C} \\
\mu_{1}+353 \rho+676.5 \rho^{2}+102.1 \rho^{3}, \text { if } T>365^{\circ} \mathrm{C}
\end{array}\right.
$$

with

$$
\mu_{1}=0.407 T+80.4
$$

where: $\rho_{v}=\operatorname{density}\left(\mathrm{g} / \mathrm{cm}^{3}\right)$

$$
T=\text { temperature }\left({ }^{\circ} \mathrm{C}\right)
$$

For temperatures between $340^{\circ} \mathrm{C}$ and $365^{\circ} \mathrm{C}$, the viscosity is linearly interpolated between values given by the two components of Equation 8.25. Viscosity is given in units of micropoise by Equation 8.25.

\subsubsection{Subcooled Liquid}

Subcooled liquid properties are found as a function of liquid enthalpy using the saturated liquid properties that have been given in Table 4. Dependence on pressure is neglected since it is very small for subcooled liquids. Density is the only term not obtained from Table 4. Specific volume is obtained using the following equation:

$$
\nu_{l}=\exp \left[\Sigma_{i=1}^{5}\left(\Sigma_{j=1}^{3} C_{C X i j} P^{j-1}\right) H_{l}^{i-1}\right]
$$


Table 9: Coefficients for the calculation of $\gamma_{r}$

\begin{tabular}{|c|c|c|}
\hline$n r$ & $j r$ & $i r$ \\
\hline$-0.17731742473213 \cdot 10^{-02}$ & 0 & 1 \\
\hline$-0.17834862292358 \cdot 10^{-01}$ & 1 & 1 \\
\hline$-0.45996013696365 \cdot 10^{-01}$ & 2 & 1 \\
\hline$-0.57581259083432 \cdot 10^{-01}$ & 3 & 1 \\
\hline$-0.50325278727930 \cdot 10^{-01}$ & 6 & 1 \\
\hline$-0.330326416702 \quad 03 \cdot 10^{-04}$ & 1 & 2 \\
\hline$-0.189489875163 \quad 15 \cdot 10^{-03}$ & 2 & 2 \\
\hline$-0.39392777243355 \cdot 10^{-02}$ & 4 & 2 \\
\hline$-0.43797295650573 \cdot 10^{-01}$ & 7 & 2 \\
\hline$-0.26674547914087 \cdot 10^{-04}$ & 36 & 2 \\
\hline $0.20481737692309 \cdot 10^{-07}$ & 0 & 3 \\
\hline $0.43870667284435 \cdot 10^{-06}$ & 1 & 3 \\
\hline$-0.32276677238570 \cdot 10^{-04}$ & 3 & 3 \\
\hline$-0.15033924542148 \cdot 10^{-02}$ & 6 & 3 \\
\hline$-0.40668253562649 \cdot 10^{-01}$ & 35 & 3 \\
\hline$-0.78847309559367 \cdot 10^{-09}$ & 1 & 4 \\
\hline $0.12790717852285 \cdot 10^{-07}$ & 2 & 4 \\
\hline $0.48225372718507 \cdot 10^{-06}$ & 3 & 4 \\
\hline $0.22922076337661 \cdot 10^{-05}$ & 7 & 5 \\
\hline$-0.16714766451061 \cdot 10^{-10}$ & 3 & 6 \\
\hline$-0.21171472321355 \cdot 10^{-02}$ & 16 & 6 \\
\hline$-0.23895741934104 \cdot 10^{+02}$ & 35 & 6 \\
\hline$-0.59059564324270 \cdot 10^{-17}$ & 0 & 7 \\
\hline$-0.12621808899101 \cdot 10^{-05}$ & 11 & 7 \\
\hline$-0.38946842435739 \cdot 10^{-01}$ & 25 & 7 \\
\hline $0.11256211360459 \cdot 10^{-10}$ & 8 & 8 \\
\hline$-0.82311340897998 \cdot 10^{+01}$ & 36 & 8 \\
\hline $0.19809712802088 \cdot 10^{-07}$ & 13 & 9 \\
\hline $0.10406965210174 \cdot 10^{-18}$ & 4 & 10 \\
\hline$-0.10234747095929 \cdot 10^{-12}$ & 10 & 10 \\
\hline$-0.10018179379511 \cdot 10^{-08}$ & 14 & 10 \\
\hline$-0.80882908646985 \cdot 10^{-10}$ & 29 & 16 \\
\hline $0.10683031879409 \cdot 10^{+00}$ & 50 & 16 \\
\hline$-0.33662250574171 \cdot 10^{+00}$ & 57 & 18 \\
\hline $0.89185845355421 \cdot 10^{-24}$ & 20 & 20 \\
\hline $0.30629316876232 \cdot 10^{-12}$ & 35 & 20 \\
\hline$-0.42002467698208 \cdot 10^{-05}$ & 48 & 20 \\
\hline$-0.59056029685639 \cdot 10^{-25}$ & 21 & 21 \\
\hline $0.37826947613457 \cdot 10^{-05}$ & 53 & 22 \\
\hline$-0.12768608934681 \cdot 10^{-14}$ & 39 & 23 \\
\hline $0.73087610595061 \cdot 10^{-28}$ & 26 & 24 \\
\hline $0.55414715350778 \cdot 10^{-16}$ & 40 & 24 \\
\hline$-0.943697072412 \quad 10 \cdot 10^{-06}$ & 58 & 24 \\
\hline
\end{tabular}


where: $C_{C X i j}=$ the coefficient based on the indices $i$ and $j$ from Table 10

The Prandtl number for subcooled water is calculated as follows:

$$
P r_{l}=\frac{\mu_{f} C p_{f}}{k_{f}}
$$

Table 10: Subcooled water density constants, $C_{C X i j}$

\begin{tabular}{|l|ccc|}
\hline$i=$ & 1 & 2 & 3 \\
\hline & & & \\
$j=1$ & $-0.413450 \cdot 10^{1}$ & $0.13252 \cdot 10^{-4}$ & $0.15812 \cdot 10^{-5}$ \\
$j=2$ & $-0.59428 \cdot 10^{-5}$ & $0.63377 \cdot 10^{-7}$ & $-0.39974 \cdot 10^{-9}$ \\
$j=3$ & $0.15681 \cdot 10^{-8}$ & $-0.40711 \cdot 10^{-10}$ & $0.25401 \cdot 10^{-12}$ \\
\hline$i=$ & 4 & 5 & \\
\hline & & & \\
$j=1$ & $-0.21959 \cdot 10^{-8}$ & $0.21683 \cdot 10^{-11}$ & \\
$j=2$ & $0.69391 \cdot 10^{-12}$ & $-0.36159 \cdot 10^{-15}$ & \\
$j=3$ & $-0.52372 \cdot 10^{-15}$ & $0.32503 \cdot 10^{-18}$ & \\
\hline
\end{tabular}

\subsection{IAPWS Water Properties}

The International Association for the Properties of Water and Steam (IAPWS) defines a consistent set of correlations to calculate the properties of water and steam that has been integrated into the code as an alternative to the above calculations. The correlations in IAPWS-IF97 [108] break up the fluid domain into several distinct regions with pressures reaching up to $100 \mathrm{MPa}$ and temperatures up to $1073 \mathrm{~K}$ for specific enthalpy, specific volume, and specific heat capacity.

Region 1 can be considered as a subcooled liquid. Region 2 can be considered as a superheated vapor. Region 3 can be considered as a supercritcal fluid. Region 5 can be considered as an extreme superheated vapor addon. The correlations for enthalpy, specific heat, and specific volume for regions 1, 2, and 5 are based off of the derivatives for the correlation of the dimensionless Gibbs free energy as a function of dimensionless pressure and temperature. Region 3 instead calculates these values based off of the derivatives for the correlations of the dimensionless Helmholtz free energy as a function of dimensionless density and temperature. The IAPWS correlations for thermal conductivity [109], dynamic viscosity [110], and surface tension [111] are not based on dimensionless free energy.

\subsection{Molten salt properties}

Thermophysical properties for $\mathrm{LiF}-\mathrm{BeF}_{2}$ (FLiBe) liquid salt are available in CTF. The properties are based on a mixture of 67 molar percent $\mathrm{LiF}$ and 33 molar percent $\mathrm{BeF}_{2}$. The implemented correlations are shown in Table 11. 


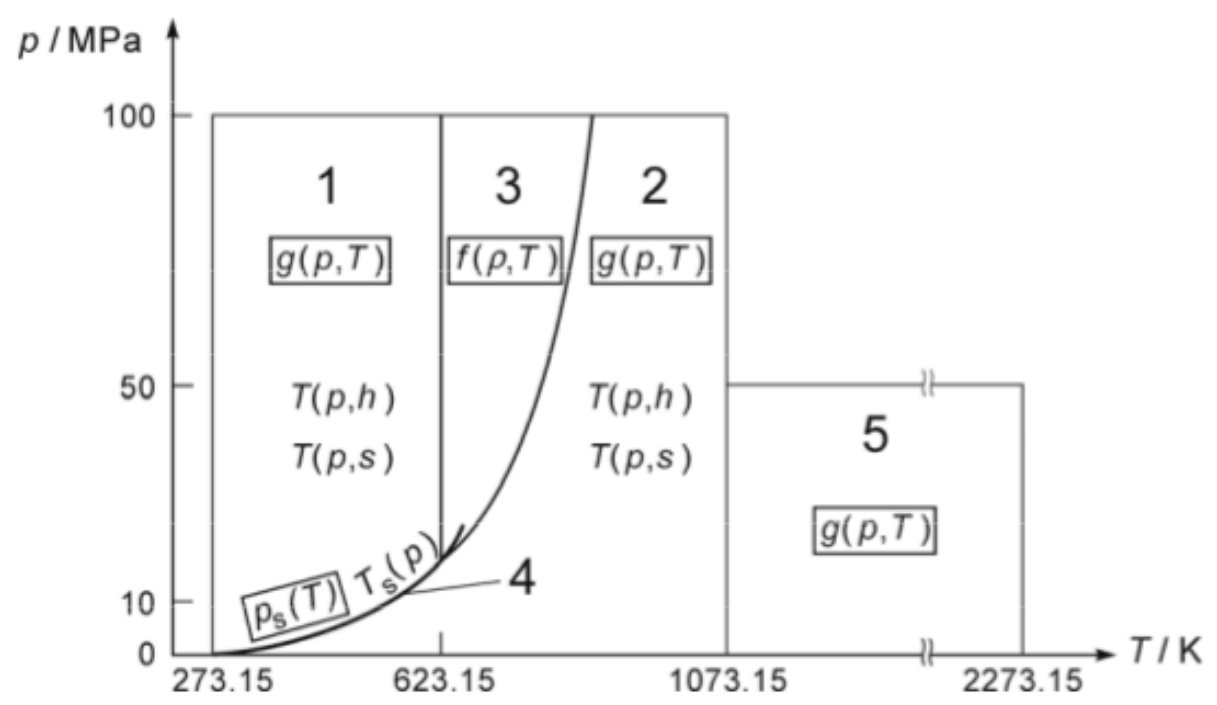

Figure 8.1: IAPWS-IF97 Fluid Regions

The liquid enthalpy relationship is defined using the assumption that the enthalpy is equal to zero at the melting temperature:

$$
h=c_{p}\left(T-T_{m e l t}\right)
$$

Standard convention would dictate that the internal energy, not the enthalpy, be set to zero at the melting temperature. The enthalpy at the melting temperature would then be calculated using the general formula:

$$
h=u+p / \rho
$$

Eq. 8.29 effectively ignores the contribution of the $p / \rho$ term to avoid introducing a pressure dependence into the enthalpy-temperature relationship, which (like the other correlations given in Table 11) is otherwise independent of pressure. The effect of this simplification is negligible, especially at the relatively low pressures typical of molten salt applications. Furthermore, only the change in enthalpy along a flow channel is of real interest, so the selection of a reference enthalpy value is only a matter of convention.

Note that CTF requires property values to be available for both the liquid and vapor phases of the working fluid; therefore, dummy values were used for the "vapor" phase of the molten salt fluid. However, the molten salt properties are intended for use only in the subcooled liquid regime, as the vapor properties are not physically realistic.

Table 11: FLiBe Salt Thermophysical Properties [112]

\begin{tabular}{|l|c|l|c|}
\hline Quantity & Equation & Units & Uncertainty \\
\hline Melting Point $\left(T_{\text {melt }}\right)$ & 731.15 & $T_{\text {melt }}$ in $\left[{ }^{\circ} \mathrm{K}\right]$ & unknown \\
\hline
\end{tabular}




\begin{tabular}{|l|c|l|c|} 
Boiling Point $\left(T_{\text {boil }}\right)$ & 1673.15 & $T_{\text {boil }}$ in $\left[{ }^{\circ} \mathrm{K}\right]$ & unknown \\
Density $(\rho)$ & $-0.4884 T+2413$. & $T$ in $\left[{ }^{\circ} \mathrm{K}\right]$ & $\pm 0.05 \%$ \\
& & $\rho$ in $\left[\mathrm{kg} / \mathrm{m}^{3}\right]$ & $\pm 0.05 \%$ \\
Dynamic Viscosity $(\mu)$ & $\left(1.16 \cdot 10^{-4}\right) e^{3755 . / T}$ & $T$ in $\left[{ }^{\circ} \mathrm{K}\right]$ & $\pm 20 \%$ \\
& $\mu$ in $[\mathrm{Pa}-\mathrm{s}]$ & $\pm 20 \%$ \\
Thermal Conductivity $(k)$ & $0.0005 T+0.63$ & $T$ in $\left[{ }^{\circ} \mathrm{K}\right]$ & $\pm 15 \%$ \\
& & $k$ in $[\mathrm{W} / \mathrm{m}-\mathrm{K}]$ & $\pm 15 \%$ \\
Specific Heat $\left(c_{p}\right)$ & 2.416 & $c_{p}$ in $[\mathrm{kJ} / \mathrm{kg}-\mathrm{K}]$ & $\pm 2 \%$ \\
\hline
\end{tabular}




\section{Solid Properites}

The primary purpose of this chapter is to provide a complete list of thermophysical solid properties available to CTF. Section 9.1 provides properties of LWR oxide fuels, Section 9.2 provides properties of fuel clad materials, and Section 9.3 provides properties used for fuel rod fill gases. The material properties correlations for the nuclear fuel rod are mostly included from recent versions of MATPRO nuclear material library handbook.

\subsection{Fuel Pellet Material Properties}

The properties are temperature dependent, so they were implemented into CTF as state functions, with a conversion from SI to English units made. The user may model other fuel types using materials besides $\mathrm{UO}_{2}$ and Zircaloy by giving CTF the thermal conductivity and specific heat as a function of material temperature in lookup tables.

\subsubsection{Fuel Emissivity}

The $\mathrm{UO}_{2}$ emissivity $\epsilon_{U O_{2}}$, used for calculating the radiative heat transfer between clad and gap. The spectral emissivity from [113], is given by

$$
\epsilon_{U O_{2}}=0.78557+1.5263 \cdot 10^{-5} \mathrm{~T}
$$

where: $T$ = fuel temperature $\left({ }^{\circ} \mathrm{K}\right)$

Figure 9.1 shows the spectral emissivity of $\mathrm{UO}_{2}$ fuel as a function of temperature with the hemispherical spectral data of Held and Wilder (1969) [114], the emissivity data of Cabannes (1967) [115], and Claudson (1958) [116].

\subsubsection{Fuel Specific Heat Capacity}

The specific heat capacity is needed for temperature calculations in transient calculations due to the calculated stored energy/enthalpy being calculated via the specific heat capacity. The specific heat of $\mathrm{UO}_{2}$ from 


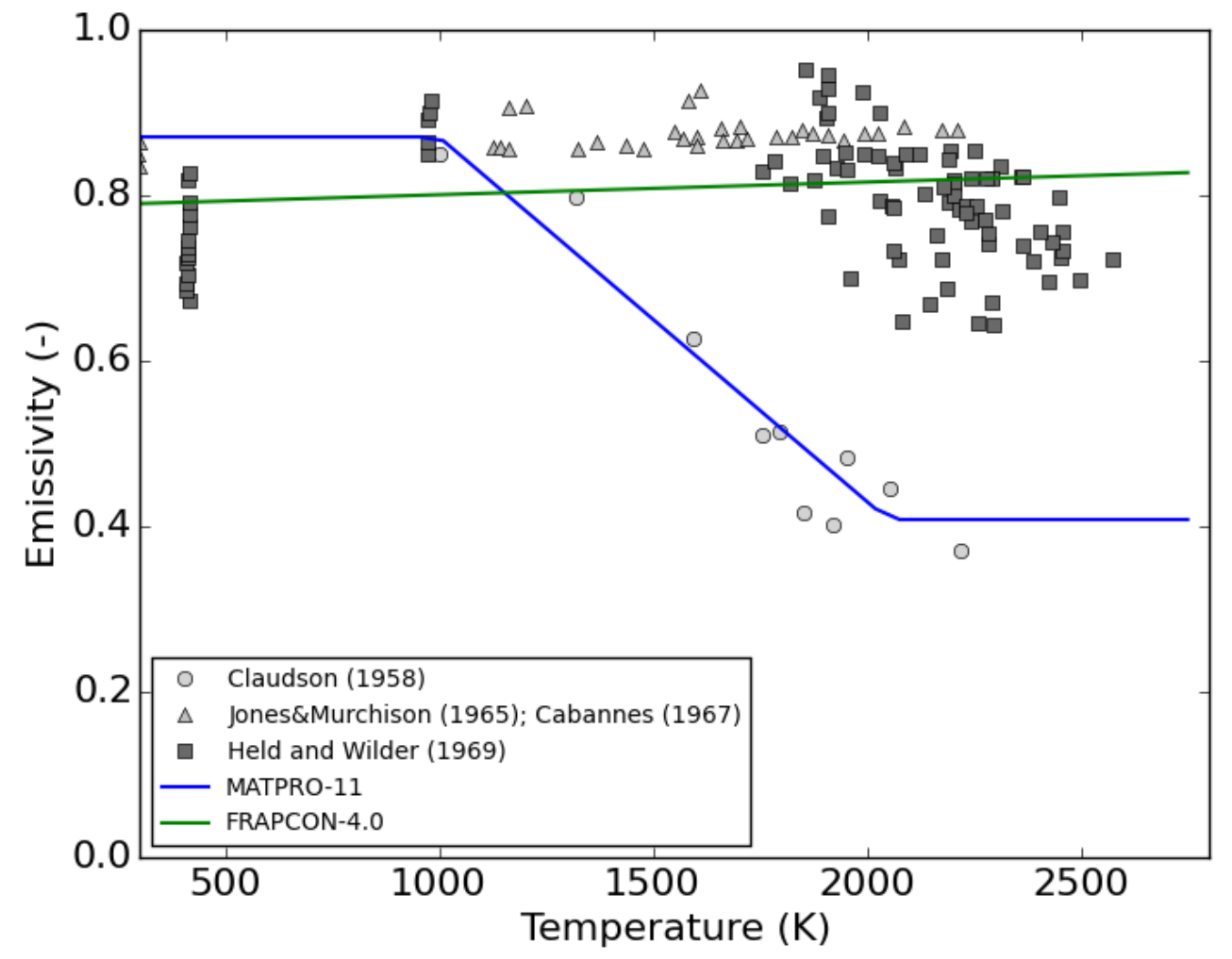

Figure 9.1: $\mathrm{UO}_{2}$ fuel emissivity. 
MATPRO-11 [117] is calculated as:

$$
C p=\left\{\frac{K_{1} \theta^{2} \exp (\theta / T)}{T^{2}(\exp (\theta / T)-1)^{2}}+K_{2} T+\frac{O M}{2} \frac{K_{3} E_{D}}{R T^{2}} \exp \left(\frac{-E_{D}}{R T}\right)\right\}
$$

where: $C p=$ specific heat capacity of $\mathrm{UO}_{2}$ fuel $\left(\mathrm{J} / \mathrm{kg}^{-}{ }^{\circ} \mathrm{K}\right)$

$T=$ the temperature ${ }^{\circ} \mathrm{K}$

$O M=$ the oxygen to metal ratio, which is taken to be 2

$R=8.3143 \mathrm{~J} / \mathrm{mol}-\mathrm{K}$

$\theta \quad=$ the Einstein temperature

Table 12: Empirical coefficients in Equation 9.2

\begin{tabular}{|c|c|c|c|c|c|}
\hline $\begin{array}{c}\text { Fuel } \\
\text { type }\end{array}$ & $\begin{array}{c}K_{1} \\
\left(\mathrm{~J} / \mathrm{kg}^{-}{ }^{\circ} \mathrm{K}\right)\end{array}$ & $\begin{array}{c}K_{2} \\
\left(\mathrm{~J} / \mathrm{kg}^{\circ} \mathrm{K}\right)\end{array}$ & $\begin{array}{c}K_{3} \\
\left(\mathrm{~J} / \mathrm{kg}^{\circ} \mathrm{K}\right)\end{array}$ & $\begin{array}{c}\theta \\
\left({ }^{\circ} \mathrm{K}\right)\end{array}$ & $\begin{array}{c}E_{D} \\
(\mathrm{~J} / \mathrm{mol})\end{array}$ \\
\hline $\mathrm{UO}_{2}$ & 296.7 & $2.43 \cdot 10^{-2}$ & $8.745 \cdot 10^{7}$ & 535.285 & $1.577 \cdot 10^{5}$ \\
\hline
\end{tabular}

Figure 9.2 shows the fuel specific heat of $\mathrm{UO}_{2}$ fuel as a function of temperature.

\subsubsection{Fuel Thermal Conductivity}

\section{Uranium Dioxide}

The cold-state density of $\mathrm{UO}_{2}$ density is $684.86 \mathrm{lbm} / \mathrm{ft}^{3}$. The thermal conductivity of $\mathrm{UO}_{2}$ is calculated in three different ways as:

- MATPRO correlation [118], with an expected operating range of $500-3000^{\circ} \mathrm{K}$, is only function of temperature. The thermal conductivity is given in units of BTU/hr-ft-F by:

$$
\begin{aligned}
K_{U O_{2}} & =C\left\{\max \left(\frac{2335}{464+T_{{ }^{\circ} C}}, 1.1038\right)\right. \\
& \left.+7.027 \cdot 10^{-3} \exp \left(1.867 \cdot 10^{-3} \cdot T_{{ }^{\circ} C}\right)\right\} \\
C & =\frac{1-\beta \cdot(1-T D)}{1-0.05 \cdot \beta} \\
\beta & =2.58-\left(5.8 \cdot 10^{-4}\right) T^{\circ}{ }_{C}
\end{aligned}
$$

where: $T_{C}=$ the temperature $\left({ }^{\circ} \mathrm{C}\right)$

$T D=$ the theoretical density $(-)$

$C, \beta=$ the empirical coefficients

This model is set to CTF's default fuel thermal conductivity model. Figure 9.3 shows the thermal conductivity of $\mathrm{UO}_{2}$ as a function of temperature. 


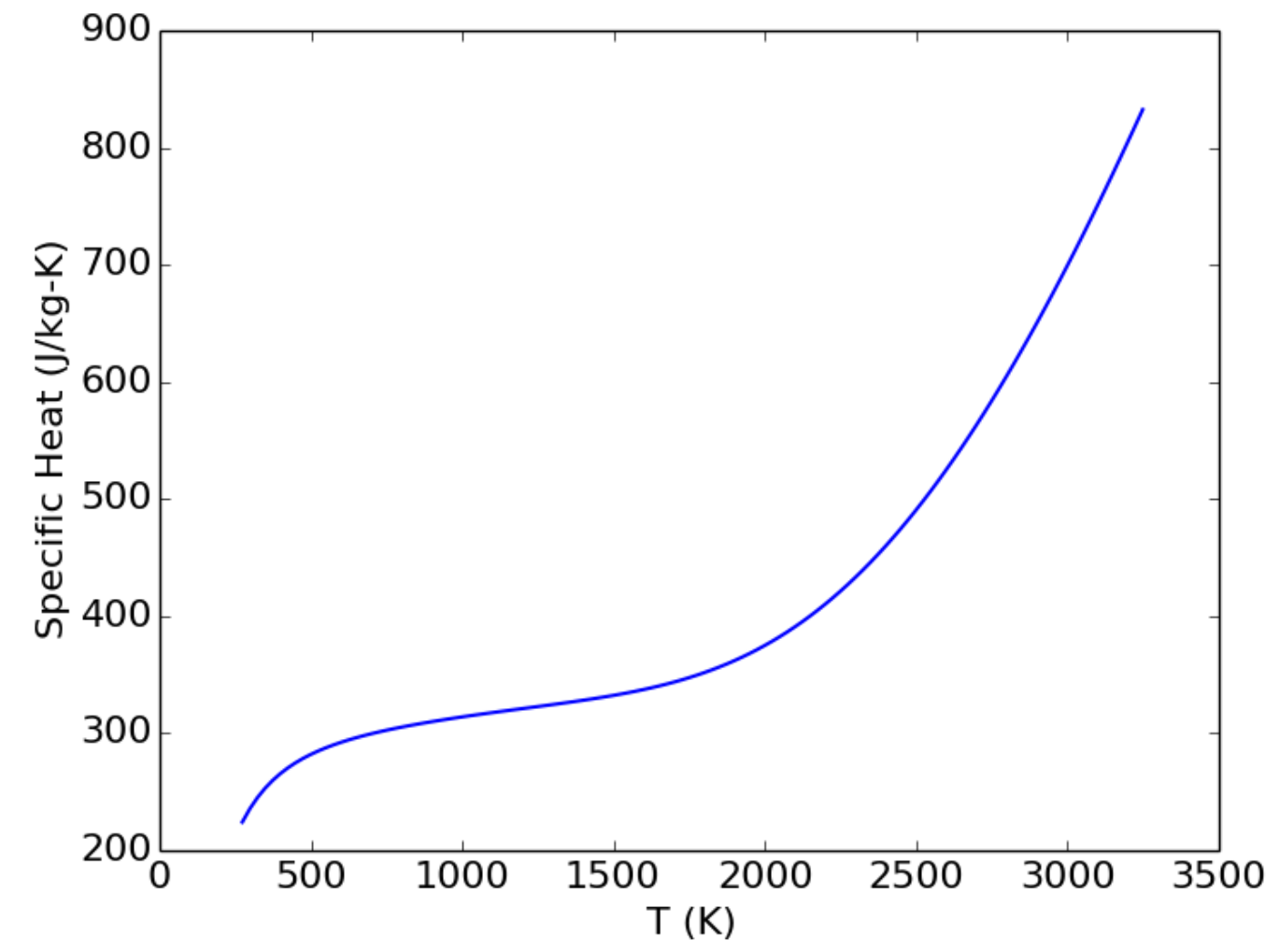

Figure 9.2: $\quad \mathrm{UO}_{2}$ fuel specific heat. 


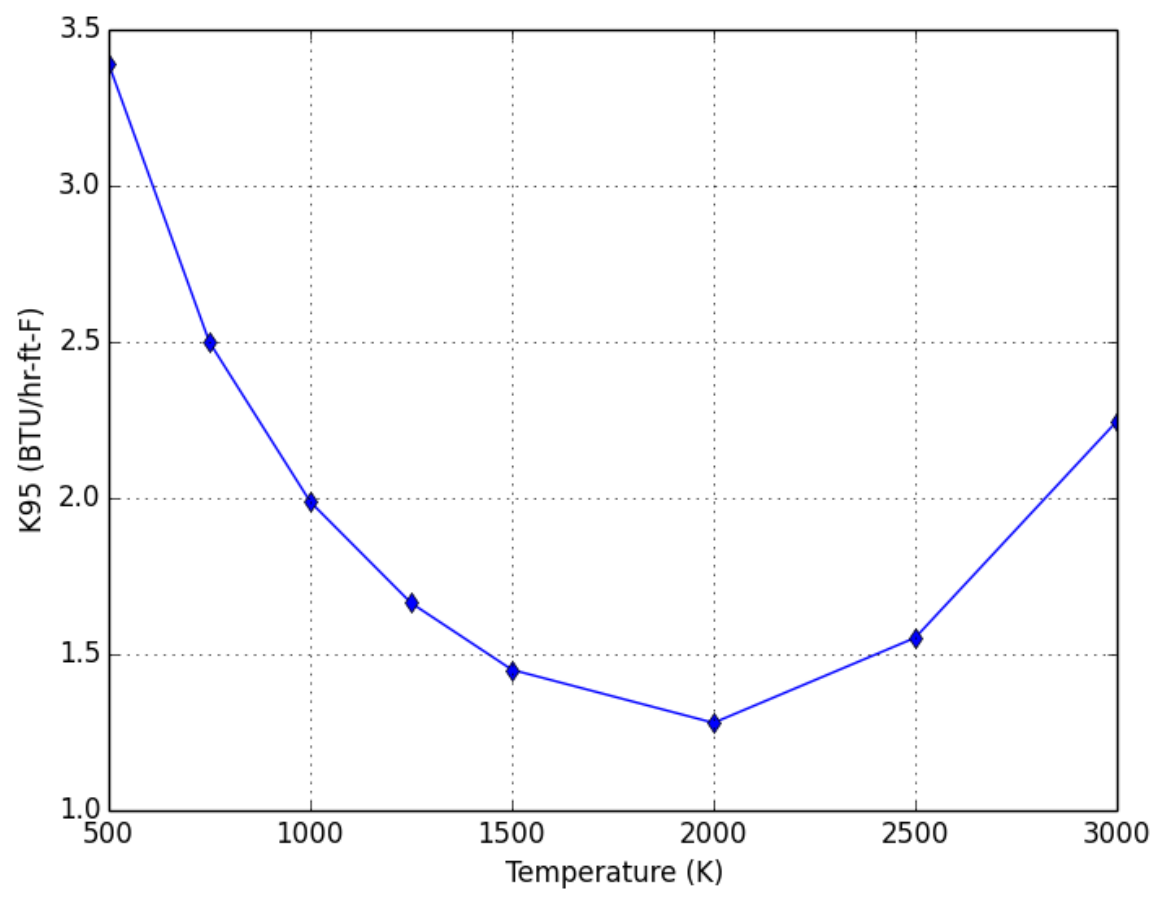

Figure 9.3: $\quad$ MATPRO-11 model for the thermal conductivity of $95 \% \mathrm{TD} \mathrm{UO}_{2}$. 
- Modified NFI correlation [119], the thermal conductivity (W/m-K) for the unirradiated $95 \%$ TD $\mathrm{UO}_{2}$ fuel is represented as the summation of a lattice vibration (phonon) term $K_{\text {phonon }}$ and electronic (electron hole pair effect) term $K_{\text {electronic }}$.

$$
\begin{aligned}
K_{U O_{2}, 95} & =K_{\text {phonon }}+K_{\text {electronic }} \\
K_{\text {phonon }} & =1 /\left(0.0452+0.000246 T_{K}+0.00187 B u+1.1599\right. \text { Gad } \\
& \left.+[1.0-0.9 \exp (-0.04 B u)] 0.038 h B u^{0.28}\right) \\
K_{\text {electronic }} & =\frac{3.50 \cdot 10^{9}}{T_{K}^{2}} \exp \left(-\frac{16361}{T_{K}}\right) \\
h & =1 /\left\{1.0+396.0 \exp \left(-\frac{6380.0}{T_{K}}\right)\right\}
\end{aligned}
$$

where: $T_{K}=$ the temperature $\left({ }^{\circ} \mathrm{K}\right)$

$B u=$ the burnup in $\mathrm{MWD} / \mathrm{kgU}$

$G a d=$ is the gadolinia content in wt.\%

$h=$ the empirical function

The model is valid over

$$
\begin{aligned}
300 & \leq T(K) \leq 3000 \\
0 & \leq B u \leq 62 \mathrm{MWd} / \mathrm{kgU} \\
0.92 & \leq T D \leq 0.97 \\
0 & \leq \text { Gad } \leq 10 \mathrm{wt} . \%
\end{aligned}
$$

Figure 9.4 shows the thermal conductivity of $95 \% \mathrm{TD} \mathrm{UO}_{2}$ as a function of temperature, burnup and gadolinia content.

- Halden correlation [120], the thermal conductivity (W/m-K) for the unirradiated $95 \% \mathrm{TD} \mathrm{UO}_{2}$ fuel is estimated as:

$$
\begin{aligned}
K_{U O_{2}, 95} & =K_{\text {phonon }}+K_{\text {electronic }} \\
K_{\text {phonon }} & =1 /\left(0.1148+1.1599 \text { Gad }+1.1599 f_{x}+4 \cdot 10^{-3} B_{U O_{2}}\right. \\
& \left.+2.475 \cdot 10^{-4}\left[1.0-0.00333 B u_{U O_{2}}\right] T_{o}\right) \\
K_{\text {electronic }} & =1.32 \cdot 10^{-2} \exp \left(0.00188 T_{C}\right) \\
T_{o} & =\min \left(1650.0, T_{C}\right)
\end{aligned}
$$

where: $T_{C} \quad=$ the temperature $\left({ }^{\circ} \mathrm{C}\right)$

$B u \quad=$ the burnup in $\mathrm{MWD} / \mathrm{kgU}$

$B u_{U O_{2}}=\mathrm{Bu} / 1.1366$, the burnup in $\mathrm{MWd} / \mathrm{kgU}$

$O M=$ oxygen-to-metal ratio (i.e., represents the deviation from stoichiometry, 2)

Gad = is the gadolinia content in wt.\%

$h \quad=$ the empirical function 


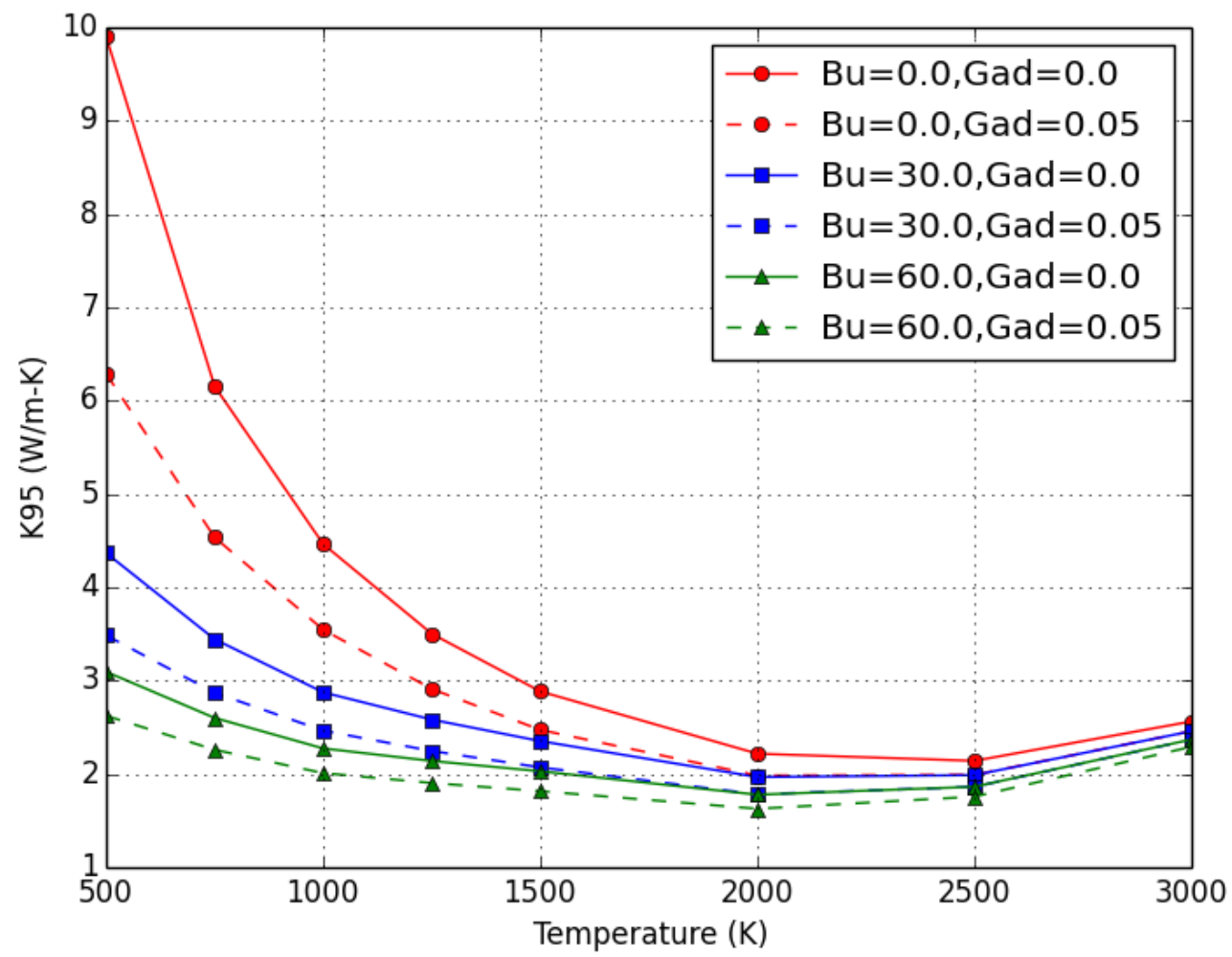

Figure 9.4: Modified NFI model for the thermal conductivity of $95 \% \mathrm{TD} \mathrm{UO}_{2}$. 
The model is valid over

$$
\begin{aligned}
300 & \leq T(K) \leq 3000 \\
0 & \leq B u \leq 62 \mathrm{MWd} / \mathrm{kgU} \\
0.92 & \leq T D \leq 0.97 \\
0 & \leq \text { Gad } \leq 10 \mathrm{wt} . \%
\end{aligned}
$$

Figure 9.5 shows the thermal conductivity of $95 \% \mathrm{TD} \mathrm{UO}_{2}$ as a function of temperature, burnup and the gadolinia content.

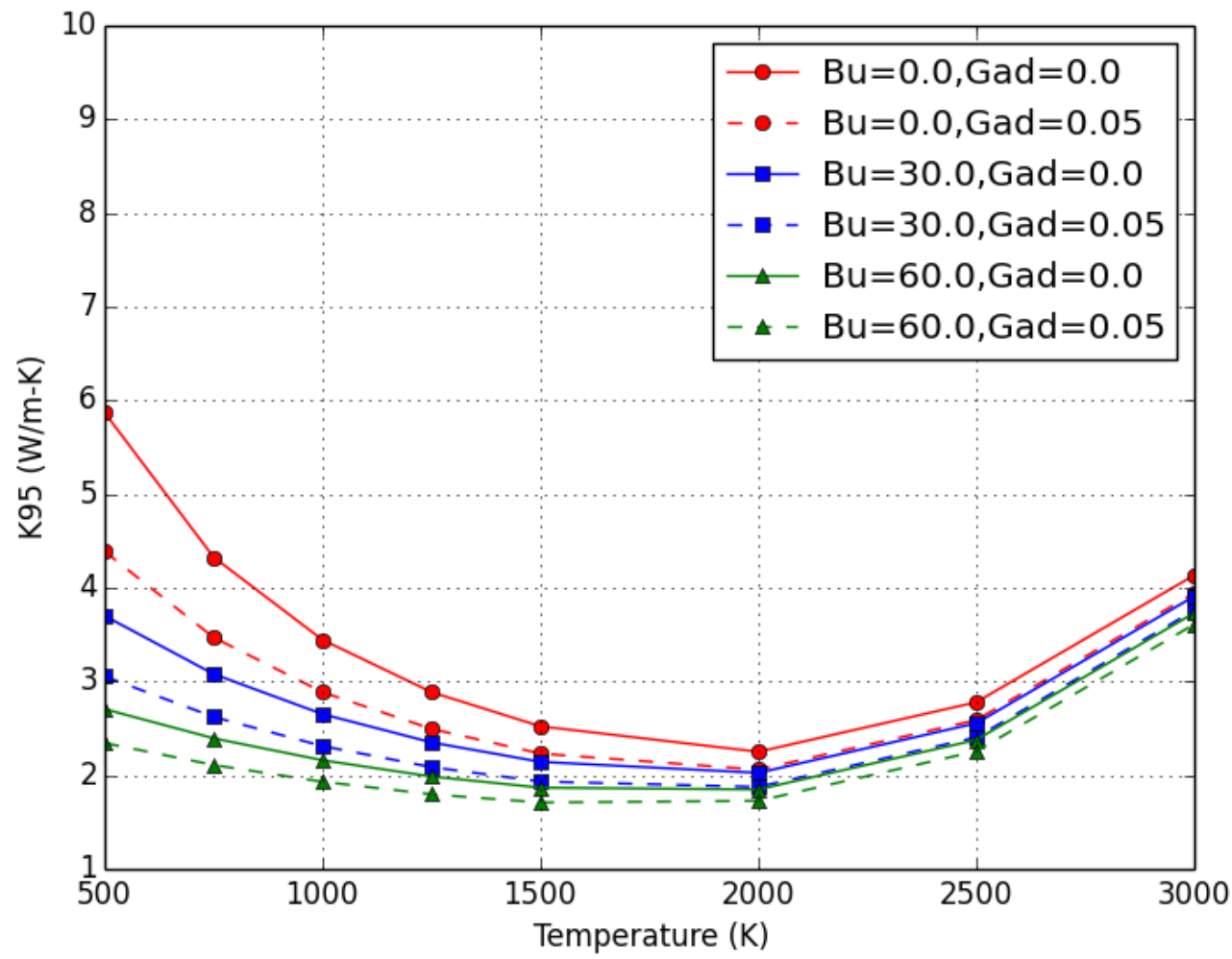

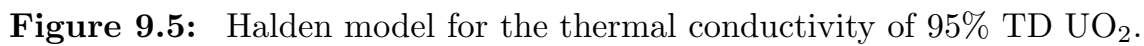

The thermal conductivity model is adjusted for as fabricated fuel density using Lucuta's recommendation for spherical-shaped pores [121, 122, 120].

$$
K_{U O_{2}}=K_{U O_{2}, 95}\left(\frac{1.0789 T D}{1.0+0.5(1.0-T D)}\right)
$$




\section{MOX}

The thermal conductivity is represented as the summation of a lattice vibration (phonon) term $K_{\text {phonon }}$ and an electronic (electron hole pair effect) term $K_{\text {electronic }}$. For unirradiated $95 \%$ TD MOX fuel, the thermal conductivity $K_{M O X, 95}$ is calculated by using three models, which are:

- The Duriez/Modified NFI correlation is developed combining the Duriez stoichiometry-dependent correlation and modified NFI correlation that includes the burnup degradation effects. For $95 \%$ TD MOX fuel, the thermal conductivity is given in units of $\mathrm{W} / \mathrm{m}-\mathrm{K}$ by

$$
\begin{aligned}
K_{M O X, 95} & =K_{\text {phonon }}+K_{\text {electronic }} \\
K_{\text {phonon }} & =1 /\left(a_{O M}+c_{O M} T_{K}+0.00187 B u+1.1599 G a d\right. \\
& \left.+[1.0-0.9 \exp (-0.04 B u)] 0.038 h B u^{0.28}\right) \\
K_{\text {electronic }} & =\frac{1.50 \cdot 10^{9}}{T_{K}^{2}} \exp \left(-\frac{13520}{T_{K}}\right) \\
h & =1 /\left\{1.0+396.0 \exp \left(\frac{-6380.0}{T_{K}}\right)\right\} \\
a_{O M} & =2.85 O M+0.035 \\
c_{O M} & =(2.86-7.15 O M) 1.0 \cdot 10^{-4}
\end{aligned}
$$

where: $a_{O M}, b_{O M}, h=$ the empirical coefficients

$O M \quad=$ oxygen-to-metal ratio

$\mathrm{Bu} \quad=$ the burnup $(\mathrm{MWD} / \mathrm{kgU})$

$T_{K} \quad=$ the temperature in $\left({ }^{\circ} \mathrm{K}\right)$

Gad $\quad=$ the gadolinia content (wt.\%)

Figure 9.6 shows the thermal conductivity of $95 \%$ TD MOX as a function of temperature, burnup and the gadolinia content.

- Halden correlation [120], the thermal conductivity for $95 \%$ TD MOX fuel is calculated as given in Equation 9.5 except $K_{\text {phonon }}$ term that is multiplied by 0.92 to account for reduction in thermal conductivity due to the presence of mixed oxides and the $K_{\text {electronic }}$ term remains unchanged.

$$
K_{M O X, 95}=0.92 K_{\text {phonon }}+K_{\text {electronic }}
$$

The model is valid over

$$
\begin{aligned}
300 & \leq T(K) \leq 3000 \\
0 & \leq B u \leq 62 \mathrm{MWd} / \mathrm{kgU} \\
0.92 & \leq T D \leq 0.97 \\
0 & \leq \text { plutonia content } \leq 7 w t . \% \\
& \quad \text { plutonia particle size }<2 \mu \mathrm{m}
\end{aligned}
$$

Figure 9.7 shows the thermal conductivity of $95 \%$ TD MOX as a function of temperature, burnup and the gadolinia content. 


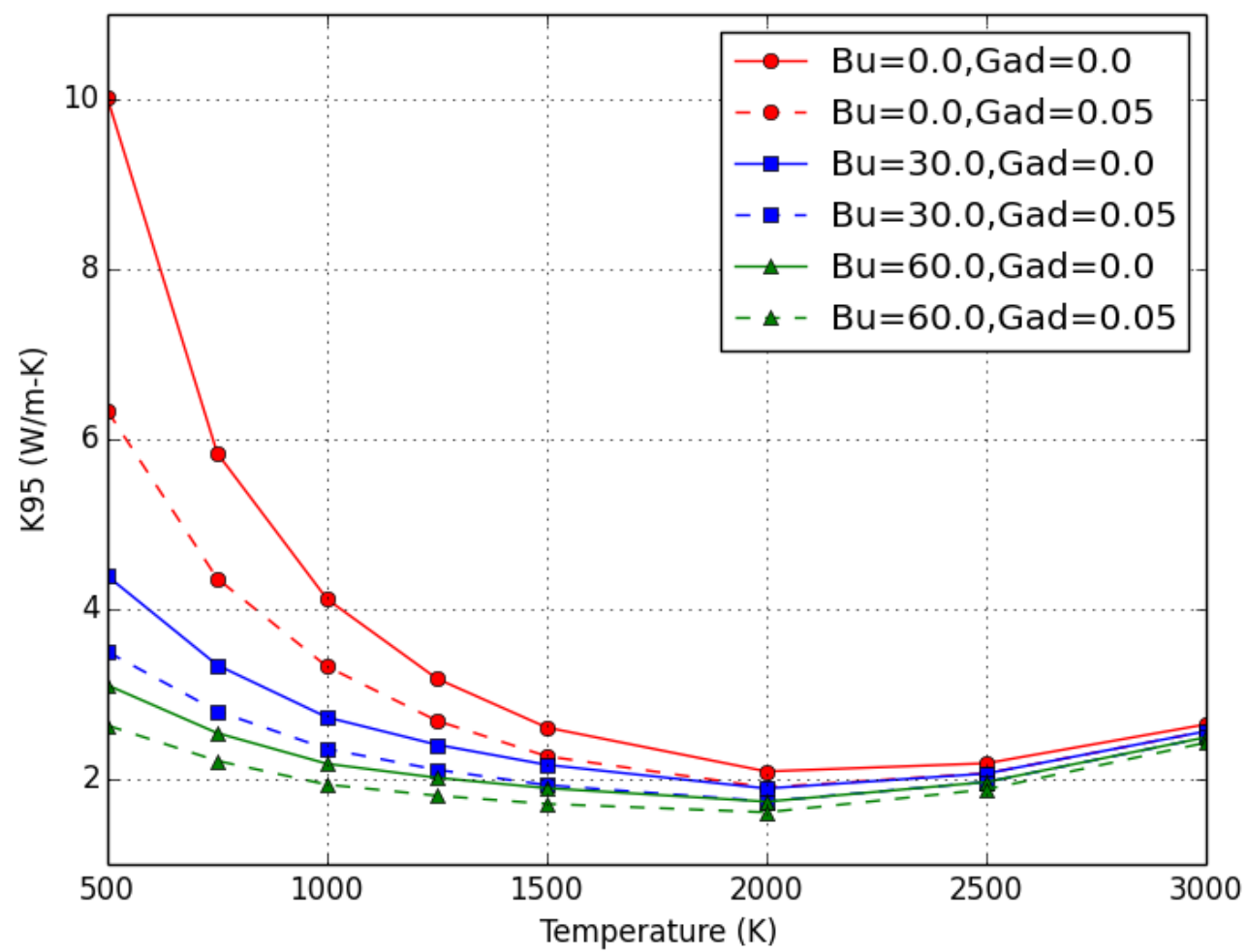

Figure 9.6: Duriez/Modified NFI model for the thermal conductivity of 95\% TD MOX. 


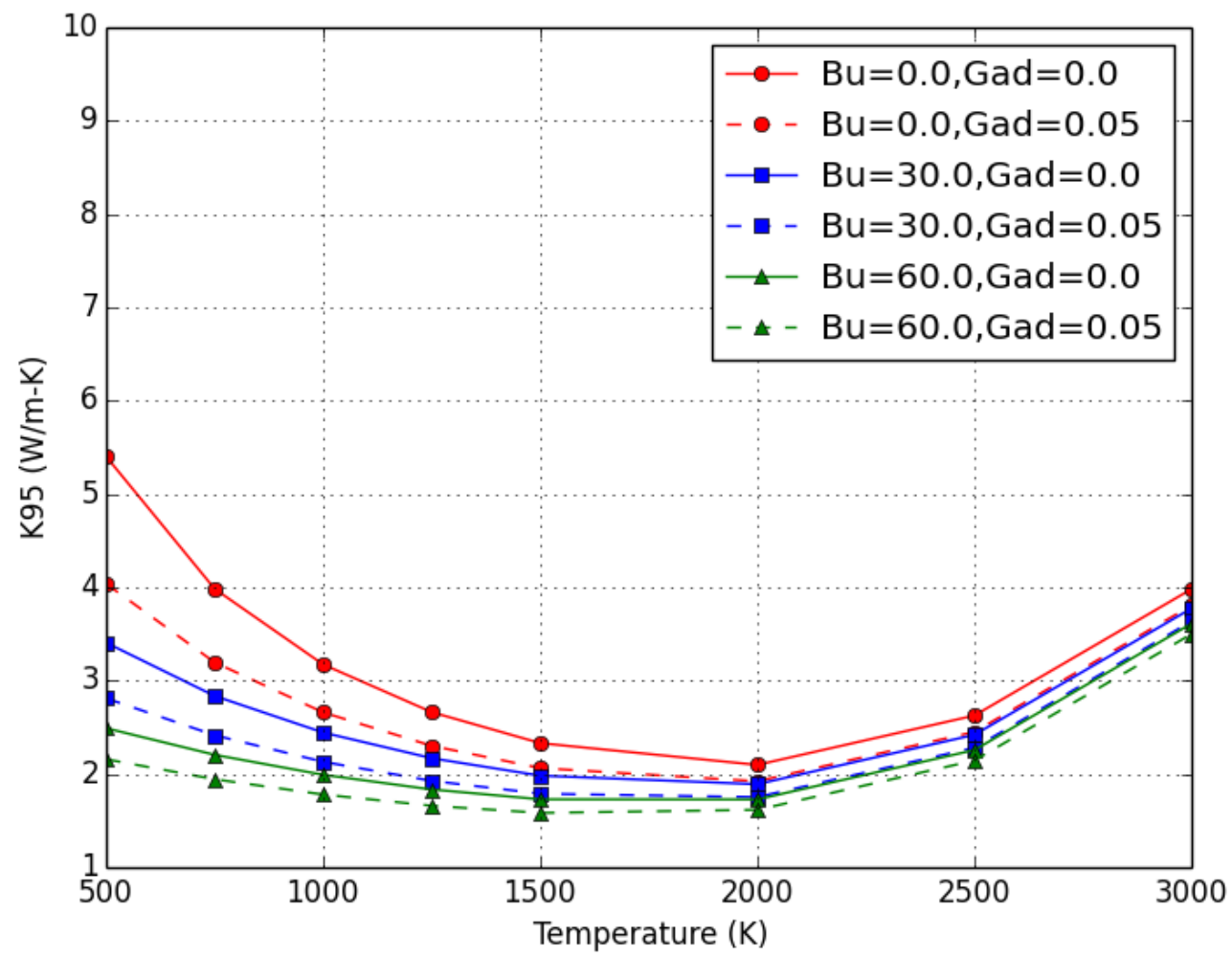

Figure 9.7: Halden model for the thermal conductivity of 95\% TD MOX. 
- Amaya correlation [123], provides a dependency on plutonium concentration by applying corrections for the $\mathrm{UO}_{2}$ thermal conductivity. CTF uses MATPRO model to compute unirradiated $\mathrm{UO}_{2}$ thermal conductivity. The MOX thermal conductivity $(\mathrm{W} / \mathrm{m}-\mathrm{K})$ is given by:

$$
\begin{aligned}
K_{M O X, 95}= & \sqrt{\frac{K_{U O_{2}, 95}}{D_{0, P u} \exp \left(D_{1, P u} T\right) y}} \cdot \\
& \arctan \left(\sqrt{D_{0, P u} \exp \left(D_{1, P u} T\right) y K_{U O_{2}, 95}}\right)
\end{aligned}
$$

where: $K_{U O_{2}, 95}=$ the unirradiated $\mathrm{UO}_{2}$ thermal conductivity $(\mathrm{W} / \mathrm{m}-\mathrm{K})$

$$
\begin{array}{ll}
T & =\text { the temperature }\left({ }^{\circ} \mathrm{K}\right) \\
y & =\text { the plutonium concentration (wt.\%) } \\
D_{0, P u}, D_{1, P u} & =\text { the empirical coefficients }
\end{array}
$$

The empirical coefficients;

$$
\begin{aligned}
& D_{0, P u}=0.209 \mathrm{~m}-\mathrm{W} / \mathrm{K} \\
& D_{1, P u}=1.09 \cdot 10^{-3} K^{-1}
\end{aligned}
$$

CTF uses MATPRO model for the thermal conductivity of $95 \% \mathrm{TD} \mathrm{UO}_{2}$. This model will be adapted to use any $\mathrm{UO}_{2}$ thermal conductivity in CTF. The MATPRO model is set for simplicity. Figure 9.8 shows the thermal conductivity of $95 \%$ TD MOX as a function of temperature, burnup and the gadolinia content.

The thermal conductivity of $100 \% T D$ is obtained by multiplying by the density correction factor to be representative of the material of interest. The thermal conductivity of MOX fuel is obtained in a similar way of $\mathrm{UO}_{2}$ as:

$$
K_{M O X}=K_{M O X, 95}\left(\frac{1.0789 T D}{1.0+0.5(1.0-T D)}\right)
$$

\subsubsection{Fuel Melting Temperature}

The fuel melting temperature is used to calculate the temperature of the liquid phase (solidus) and the last solid phase (liquidus) of $\mathrm{UO}_{2}$ and MOX fuels as a function of burnup, gadolina content and plutonia content $[117,113]$.

$$
\begin{aligned}
T_{\text {sldus }} & =3113.15-5.41395 X_{P u}+7.46839 \cdot 10^{-3} X_{P u}^{2} \\
T_{\text {liqdus }} & =3113.15-3.21860 X_{P u}+1.448518 \cdot 10^{-2} X_{P u}^{2}
\end{aligned}
$$

where: $T=$ fuel local temperature $\left({ }^{\circ} \mathrm{K}\right)$

$X_{P u}=$ the content of plutonia [wt.\%] 


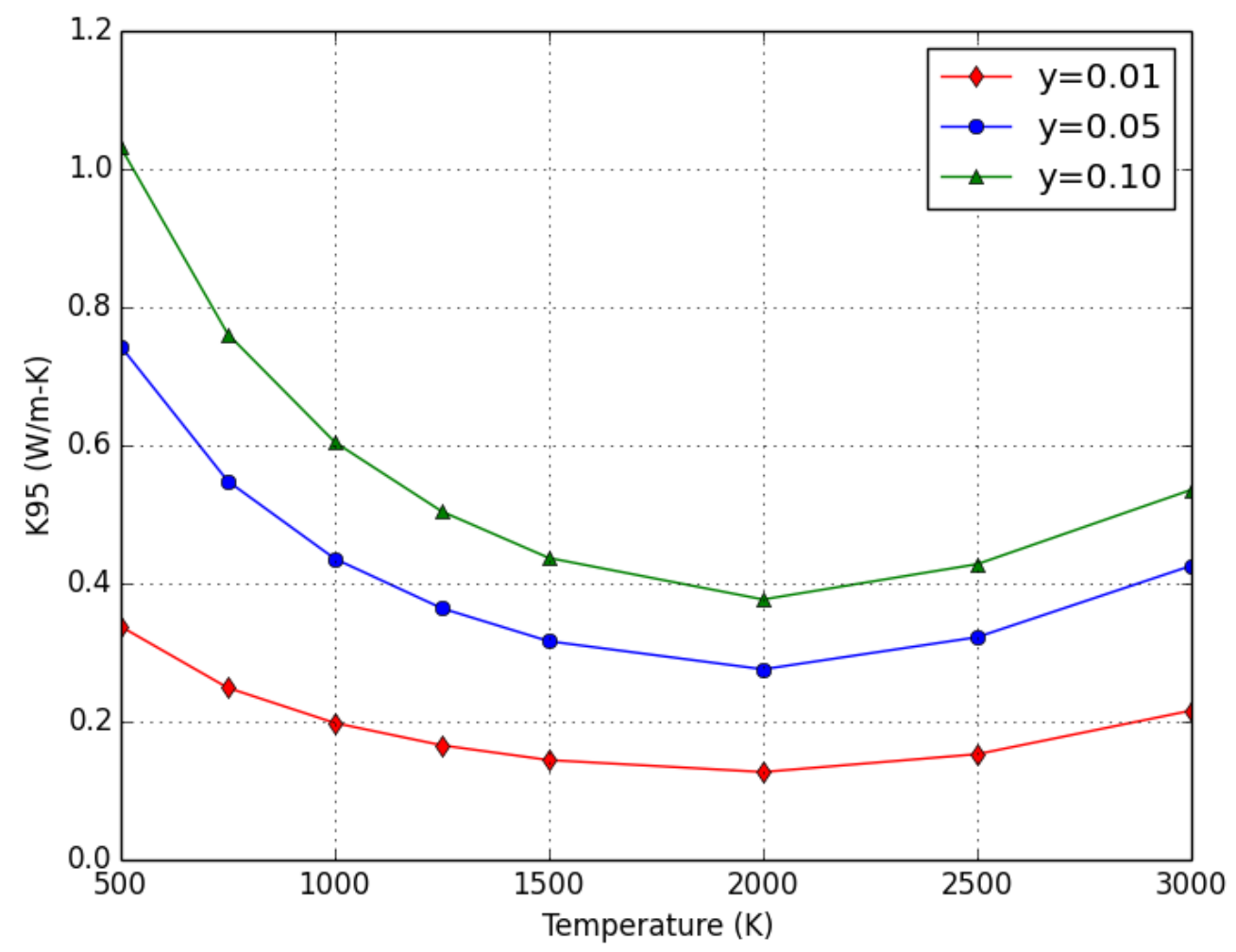

Figure 9.8: Amaya model for the thermal conductivity of 95\% TD MOX. 
The melting temperature is calculated as:

- for $X_{P u}=0$ :

$$
T_{m}=3113.15-5.0 \cdot 10^{-4} B u
$$

- for $X_{P u} \neq 0$ :

$$
\begin{aligned}
T_{m} & =T_{\text {sldus }}-5.0 \cdot 10^{-4} B u \\
\Delta T_{m} & =T_{\text {liqdus }}-T_{\text {sldus }}-5.0 \cdot 10^{-4} B u
\end{aligned}
$$

- for Gd-doped urania:

$$
T_{m}=3115-4.8 X_{G d_{2} O_{3}}
$$

where: $B u=$ fuel burnup $[\mathrm{MWd} / \mathrm{MTU}]$

$X_{G_{2} \mathrm{O}_{3}}=$ the concentration of gadolinia [wt.\%]

$T_{\text {sldus }}=$ solidus temperature $[\mathrm{K}]$

$T_{\text {liqdus }}=$ liquidus temperature $[\mathrm{K}]$

$T_{m} \quad=$ fuel melting temperature $[\mathrm{K}]$

$\Delta T_{m} \quad=$ temperature range between the solidus and liquidus $[\mathrm{K}]$

Figure 9.9 shows the solidus and liquidus boundaries of the melting temperature of $\mathrm{UO}_{2}$ and $\mathrm{MOX}$ as well as the calculated fuel melting temperature.

\subsubsection{Fuel Thermal Expansion}

The FTHEXP subroutine to calculate the fuel thermal expansion is used in CTF [117, 113]. Linear strain caused by fuel thermal expansion (equal to zero at $300 \mathrm{~K}$ ) is given in a generic form of

$$
\left(\frac{\Delta L}{L}\right)=K_{1} T-K_{2}+K_{3} \exp \left(-E_{D} / k T\right)
$$

- During melting, an expansion equal to a linear strain of 0.043 occurs.

- For the fuel is partially molten:

$$
\left(\frac{\Delta L}{L}\right)=\left(\frac{\Delta L}{L}\right)_{T_{m}}+0.043 \cdot R_{\text {molten }}
$$

- For the entirely molten fuel:

$$
\left(\frac{\Delta L}{L}\right)=\left(\frac{\Delta L}{L}\right)_{T_{m}}+0.043+3.5 \cdot 10^{-5}\left[T-\left(T_{m}+\Delta T_{m}\right)\right]
$$



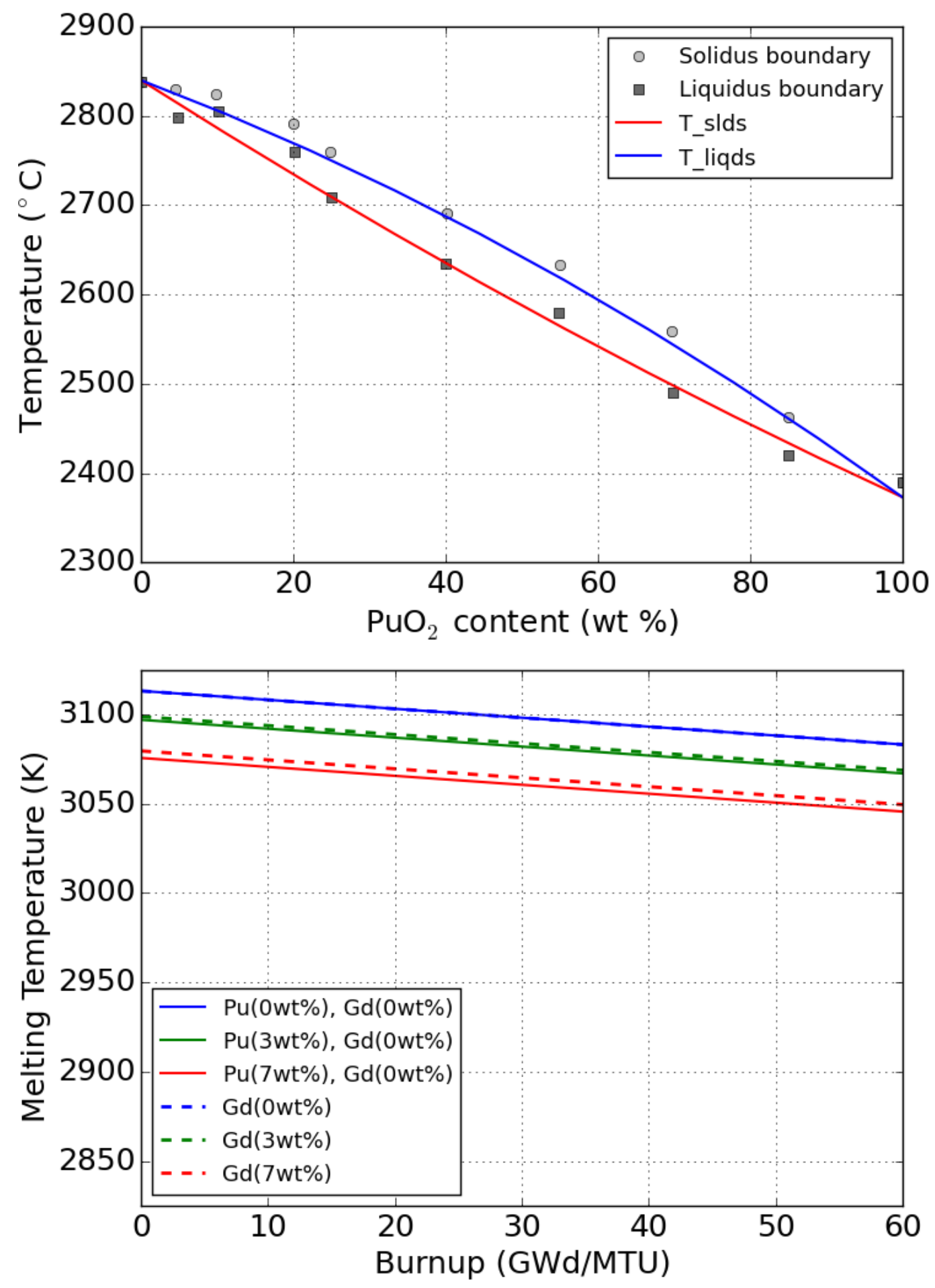

Consortium for Advanced Simulation of LWRs $\quad 226$

Figure 9.9: Solidus and liquidus boundaries of the melting temperature (top) and melting temperature (bottom). 
where: $(\Delta L / L)_{T_{m}}=$ thermal expansion of solid fuel with $T=T_{m}[-]$

$E_{D} \quad=$ energy of formation of a defect $[\mathrm{J}]$

$k \quad=$ Boltzmann's constant $\left[1.38 \cdot 10^{-23} \mathrm{~J} / \mathrm{K}\right]$

$R_{\text {molten }}=$ fraction of the molten fuel [-] (solid 0.0; molten 1.0)

$T \quad=$ temperature $[\mathrm{K}]$

$T_{m} \quad=$ fuel melting temperature $[\mathrm{K}]$

The empirical constants for $\mathrm{UO}_{2}$ and $\mathrm{PuO}_{2}$ to be used in Equation 9.16 are tabulated in Table 13.

Table 13: The empirical constants for $\mathrm{UO}_{2}$ and $\mathrm{PuO}_{2}$ (See Equation 9.16)

\begin{tabular}{|l|c|c|c|}
\hline Constant & $\begin{array}{c}\text { MATPRO } \\
\mathbf{U O}_{2}\end{array}$ & $\begin{array}{c}\text { FRAP } \\
\mathbf{U O}_{2}\end{array}$ & $\mathbf{P u O}_{2}$ \\
\hline$K_{1}\left[\mathrm{~K}^{-1}\right]$ & $1.0 \cdot 10^{-5}$ & $9.80 \cdot 10^{-6}$ & $9.0 \cdot 10^{-6}$ \\
$K_{2}[-]$ & $3.0 \cdot 10^{-3}$ & $2.61 \cdot 10^{-3}$ & $2.7 \cdot 10^{-3}$ \\
$K_{3}[-]$ & $4.0 \cdot 10^{-2}$ & $3.16 \cdot 10^{-1}$ & $7.0 \cdot 10^{-2}$ \\
$E_{D}[\mathrm{~J}]$ & $6.9 \cdot 10^{-20}$ & $1.32 \cdot 10^{-19}$ & $7.0 \cdot 10^{-20}$ \\
\hline
\end{tabular}

Figure 9.10 and 9.11 show the linear strain caused by the thermal expansion of $\mathrm{UO}_{2}$ and $\mathrm{PuO}_{2}$ fuels, respectively, as a function of fuel temperature.

\subsection{Cladding Material Properties}

\subsubsection{Cladding Thermal Conductivity}

The cold-state density of Zircaloy is $409 \mathrm{lbm} / \mathrm{ft}^{3}$. The thermal conductivity of $\mathrm{Zr} 2 / 4$ is calculated using model from MATPRO-11 [117] as follows:

$$
K_{Z}=7.51+0.0209 T-1.45 \cdot 10^{-5} T^{2}+7.67 \cdot 10^{-9} T^{3}
$$

where: $K_{Z}=$ thermal conductivity $(\mathrm{W} / \mathrm{m}-\mathrm{K})$

$T$ = fuel temperature $\left({ }^{\circ} \mathrm{K}\right)$

Thermal conductivity of zircaloy, $K_{Z}$ (W/m-K) from FRAPCON [113] is given as:

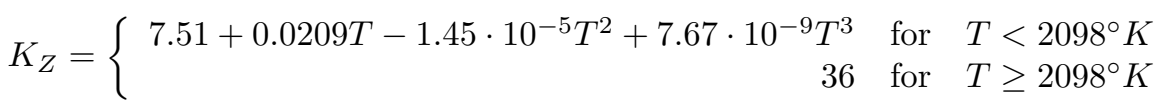

Thermal conductivity of zircaloy, $K_{Z}(\mathrm{~W} / \mathrm{m}-\mathrm{K})$ from IAEA report [124] is given as: 


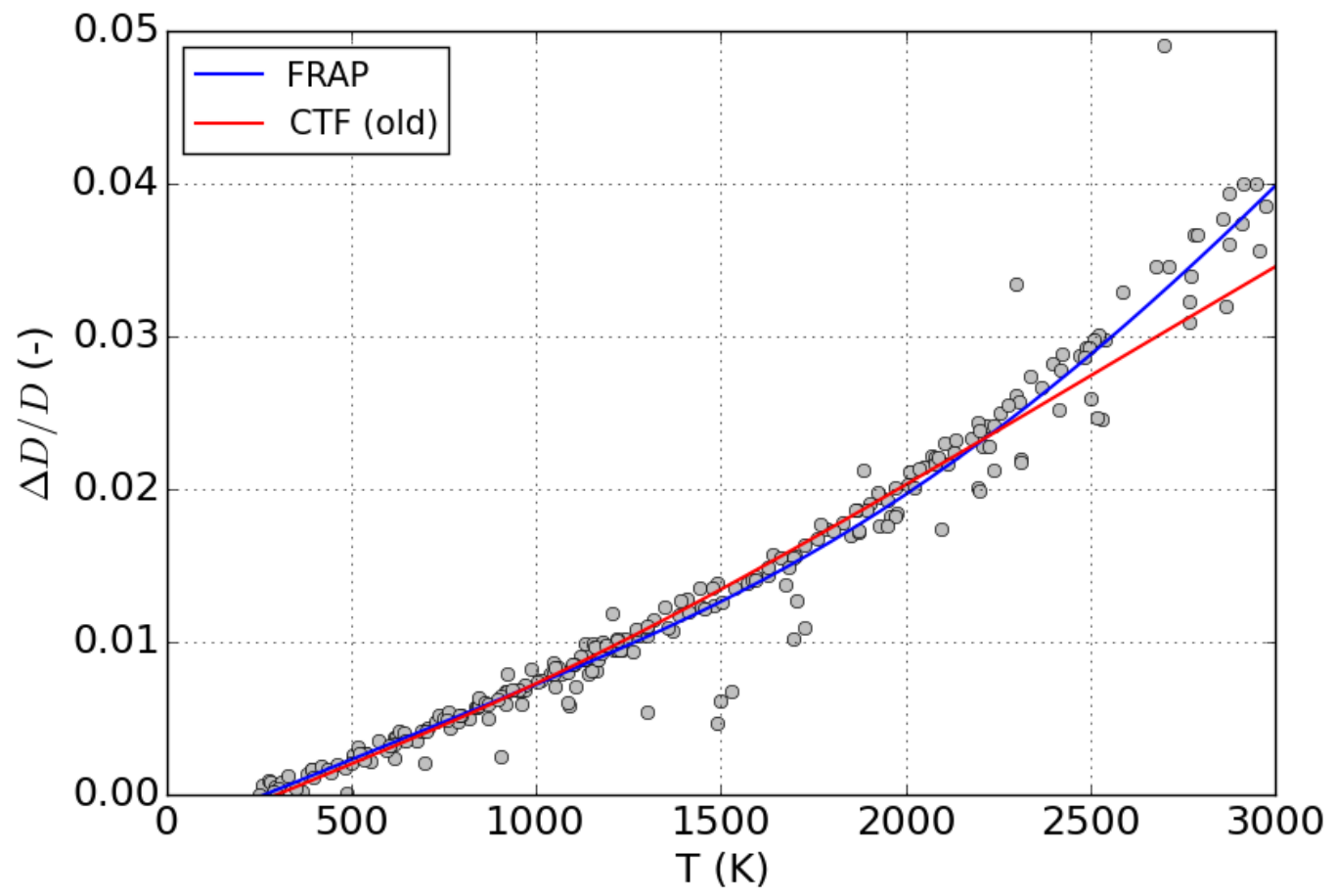

Figure 9.10: $\mathrm{UO}_{2}$ fuel thermal expansion. 


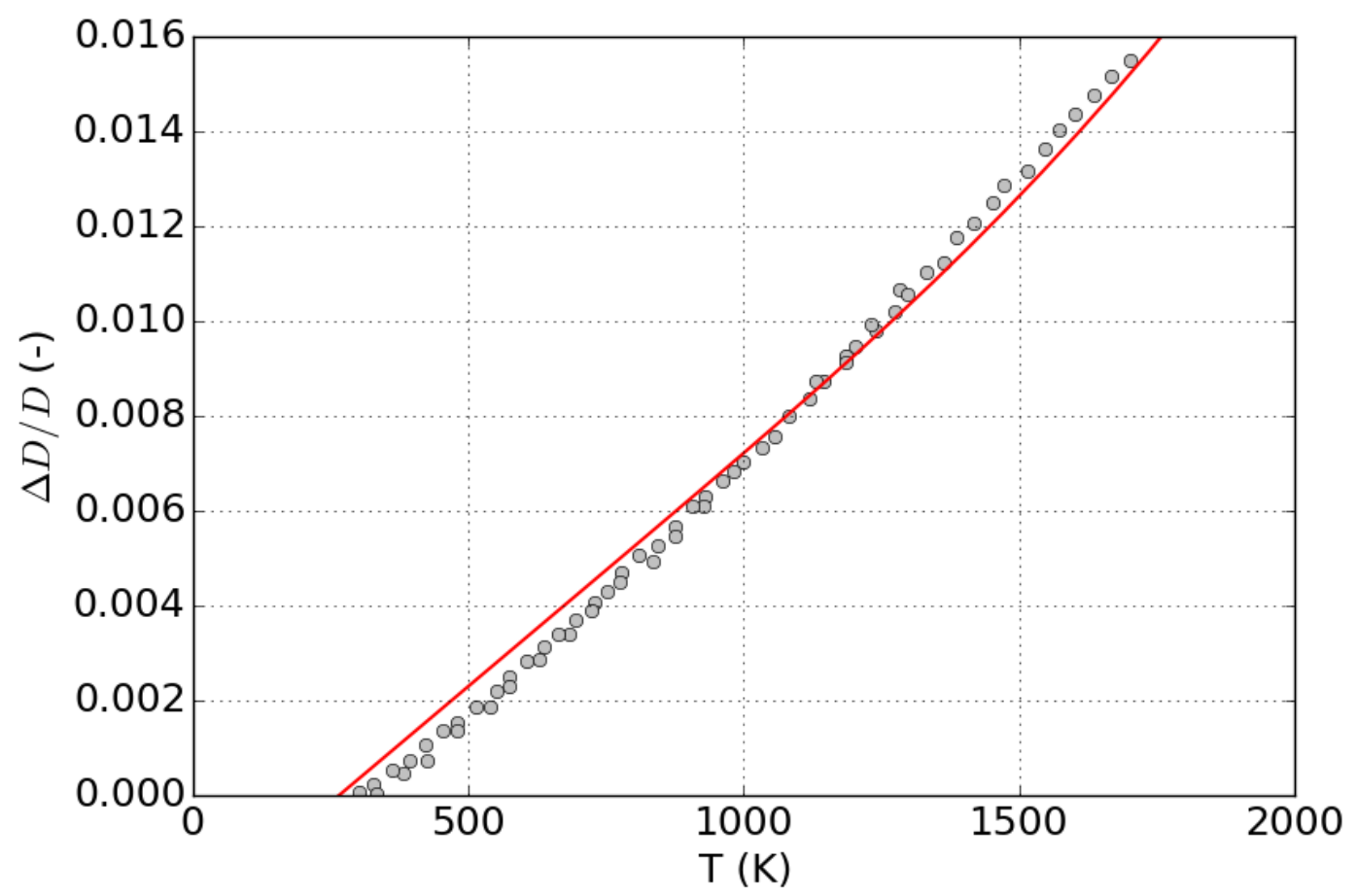

Figure 9.11: $\mathrm{PuO}_{2}$ fuel thermal expansion. 


$$
K_{Z}=12.767-5.4348 \cdot 10^{-4} T+8.9818 \cdot 10^{-6} T^{2}
$$

Add table for experimental data here !!

Figure 9.12 shows the thermal conductivity of zircaloy as a function of temperature.

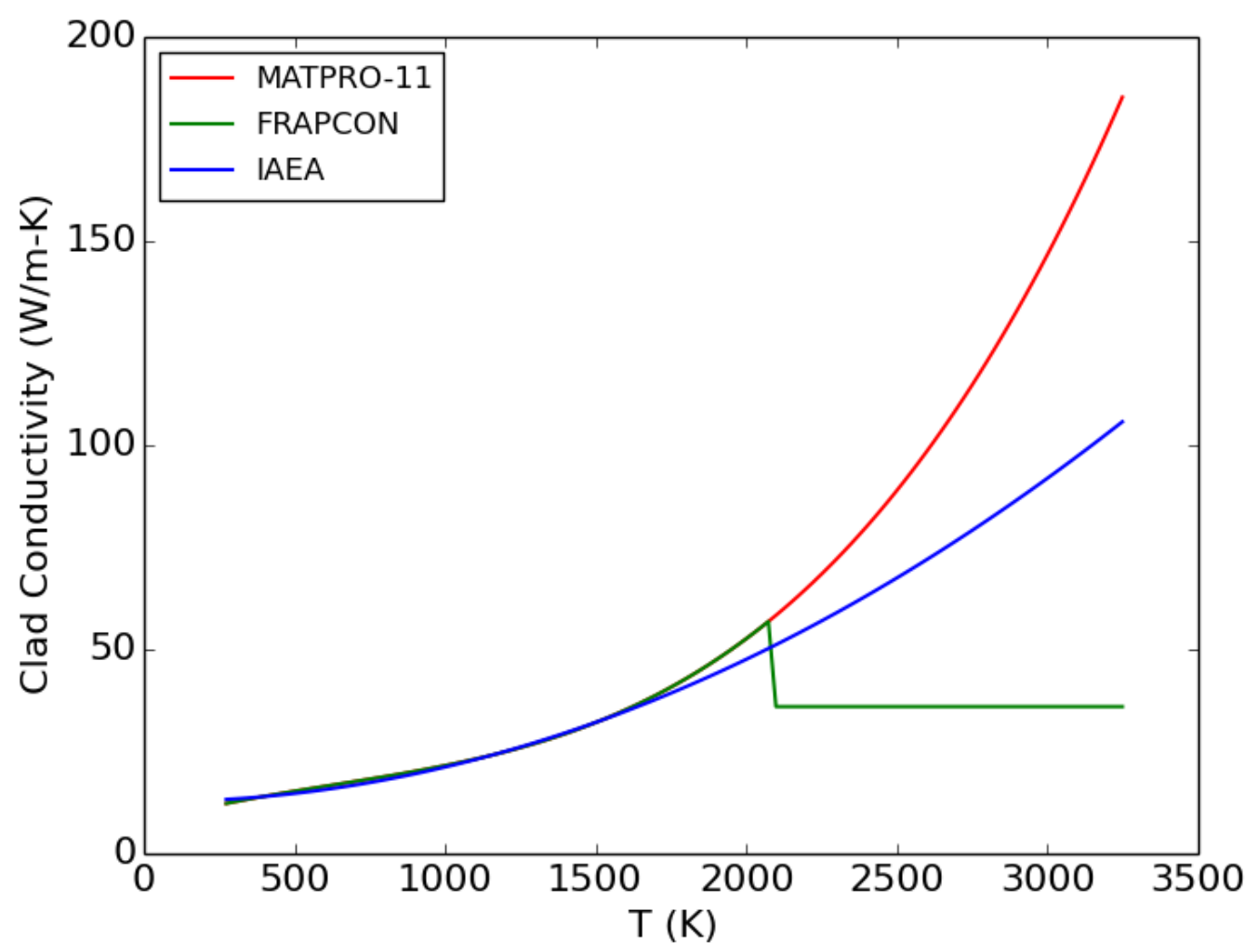

Figure 9.12: Thermal conductivity of zircaloy.

\subsubsection{Cladding Oxide Thermal Condutivity}

The cold-state density of $\mathrm{ZrO}_{2}$ is $409 \mathrm{lbm} / \mathrm{ft}^{3}$ and the specific heat capacity of $\mathrm{ZrO}_{2}$ is set to 0.0785 . The thermal conductivity, $K_{\mathrm{ZrO}_{2}}(\mathrm{~W} / \mathrm{m}-\mathrm{K})$ as a function of temperature $T\left({ }^{\circ} \mathrm{K}\right)$ from $[117,113]$ is given as:

$$
K_{\mathrm{ZrO}_{2}}=1.96-2.41 \cdot 10^{-4} T+6.43 \cdot 10^{-7} T^{2}-1.95 \cdot 10^{-10} T^{3}
$$

where: $\mathrm{K}_{\mathrm{ZrO}_{2}}=$ thermal conductivity $(\mathrm{W} / \mathrm{m}-\mathrm{K})$

$T=$ fuel temperature $\left({ }^{\circ} \mathrm{K}\right)$ 
Figure 9.13 shows the thermal conductivity of $\mathrm{ZrO}_{2}$ as a function of temperature.

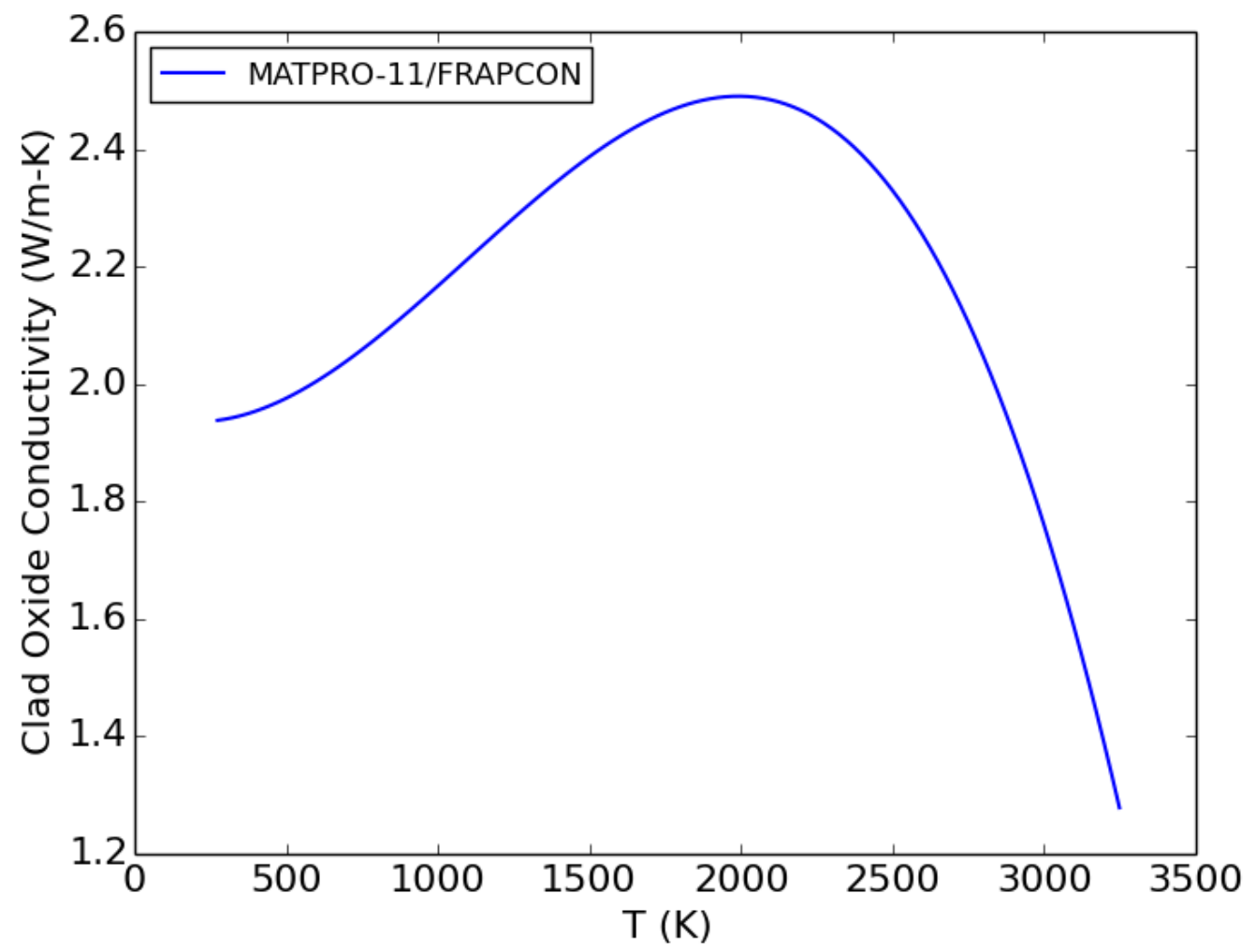

Figure 9.13: Thermal conductivity of $\mathrm{ZrO}_{2}$.

\subsubsection{Cladding Specific Heat}

The specific heat of Zircaloy is obtained from a lookup table taken from MATPRO-11 (Revision 1)[62], shown in Table 14. Figure 9.14 shows the tabulated data of cladding specific heat as a function of temperature with $\alpha$-phase data of Brooks and Stansbury (1966) [125] and $\beta$-phase data of Deem and Eldridge (1967) [126] and Coughlin and King (1950) [127]. The data of cladding specific heat are applicable for Zircaloy-2, Zircaloy-4, ZIRLO, Optimized ZIRLO, and M5. 


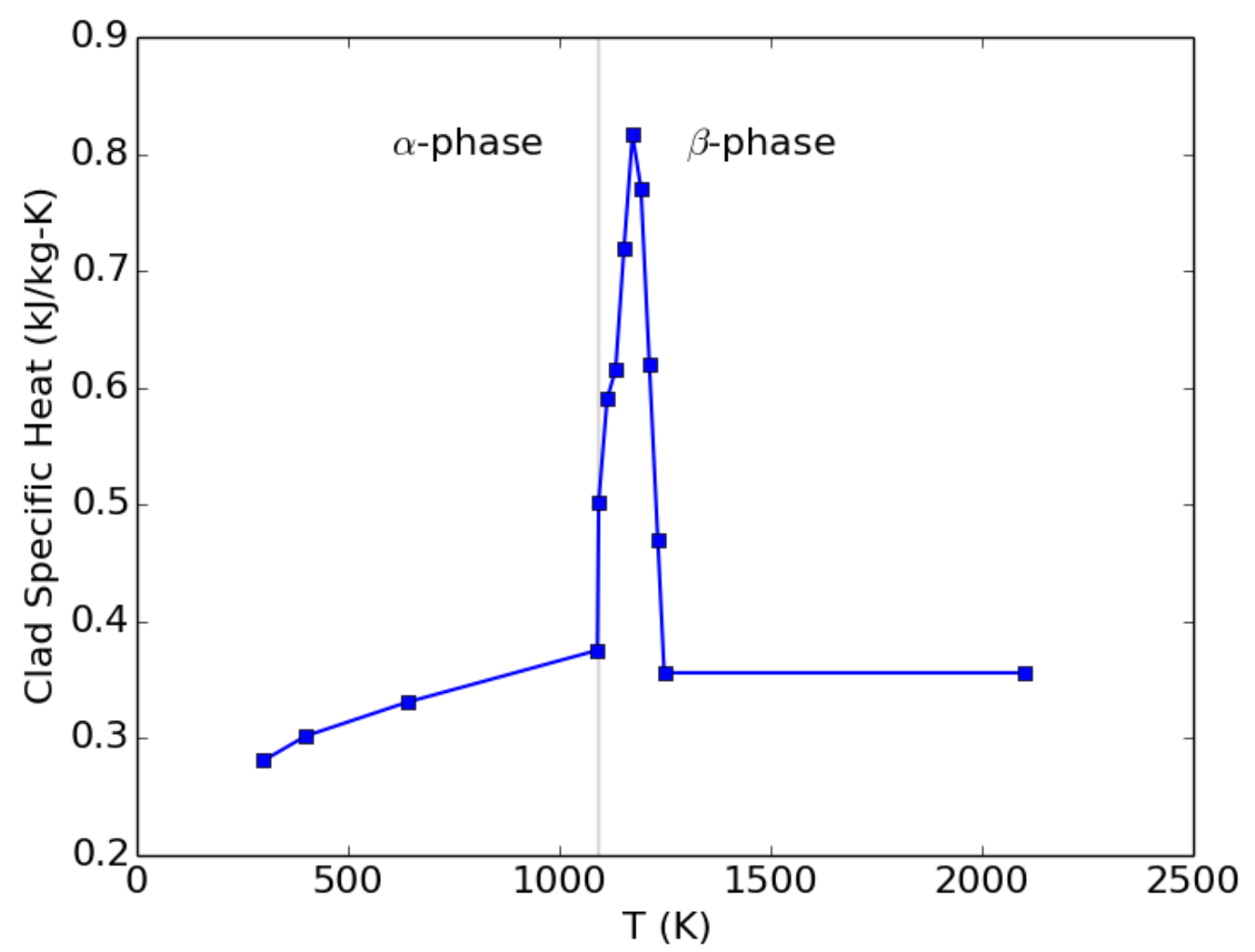

Figure 9.14: Cladding specific heat capacity.

Table 14: Lookup table for calculating the specific heat of Zircaloy

\begin{tabular}{ccc}
\hline $\mathbf{T}\left({ }^{\circ} \mathbf{K}\right)$ & $\mathbf{C p}(\mathbf{J} / \mathbf{k g - K})$ & Source \\
\hline 300 & 281 & Brooks and Stansbury $[125]$ \\
400 & 302 & \\
640 & 331 & \\
1090 & 375 & \\
\hline 1093 & 502 & Deem and Eldridge [126] \\
1113 & 590 & \\
1133 & 615 & \\
1153 & 719 & \\
1173 & 816 & \\
1193 & 770 & \\
1213 & 619 & \\
1233 & 469 & \\
1248 & 356 & \\
2098 & 356 & Coughlin and King $[127]$ \\
2099 & 356 & \\
\hline
\end{tabular}




\subsubsection{Cladding Surface Emissivity}

The emissivity of the zirconium cladding with thin oxide coatings was taken from the ZOEMIS subroutine of MATPRO-11 (Revision 2)[117], which is given as:

$$
\epsilon_{Z, 1}=\left\{\begin{aligned}
0.325+0.1246 \cdot 10^{6} d & \text { for } \quad d<3.88 \times 10^{-6} \\
0.808642-50 d & \text { for } \quad d \geq 3.88 \times 10^{-6}
\end{aligned}\right.
$$

where: $d=$ the oxide thickness $(\mathrm{m})$

$T=$ the maximum cladding temperature $\left({ }^{\circ} \mathrm{K}\right)$

When the maximum cladding temperature exceeds $1500^{\circ} \mathrm{K}$, the emissivity is assumed to be greater than 0.325. Equation 9.23 relates the emissivity $\epsilon_{Z, 2}$ (unitless) above $1500^{\circ} \mathrm{K}$ to $\epsilon_{Z, 1}$ and $T\left({ }^{\circ} \mathrm{K}\right.$ ) as:

$$
\epsilon_{Z, 2}=\epsilon_{Z, 1} \exp \left(\frac{[1500-T]}{300}\right)
$$

Figure 9.15 shows the cladding spectral emissivity of zirconium as a function of temperature and oxide thickness.

\subsubsection{Cladding Meyer Hardness}

For the case when the gap closes due to fuel pellet expansion, there will be fuel/cladding contact. In this case, it is necessary to know the hardness of the cladding for determining the heat transfer due to pellet/clad contact. Meyer hardness as a function of temperature, $T\left({ }^{\circ} \mathrm{K}\right), H_{Z}\left(\mathrm{~N} / \mathrm{m}^{2}\right)$ is implemented to CTF from the CMHARD subroutine of MATPRO-11 manual [117], and given as:

$$
H_{Z}=\exp \left(26.03-0.02639 T+4.3502 \cdot 10^{-5} T^{2}-2.5621 \cdot 10^{-8} T^{3}\right)
$$

where: $T=$ temperature $\left({ }^{\circ} \mathrm{K}\right)$

Figure 9.16 shows the cladding Meyer hardness as a function of temperature with the data of Peggs and Godin (1975) [128].

\subsubsection{Cladding Thermal Expansion}

For determining the cladding deformation due to thermal expansion, it's important to know the cladding strain and stress. The cladding strain is calculated in axial and radial directions.

For the $\alpha$-phase runs with temperatures between $300^{\circ} \mathrm{K}$ and $1073^{\circ} \mathrm{K}$ :

$$
\begin{aligned}
\epsilon_{\mathrm{Z}, \text { axial }} & =4.44 \cdot 10^{-6} \mathrm{~T}-1.24 \cdot 10^{-3} \\
\epsilon_{\mathrm{Z}, \text { radial }} & =6.72 \cdot 10^{-6} \mathrm{~T}-2.07 \cdot 10^{-3}
\end{aligned}
$$




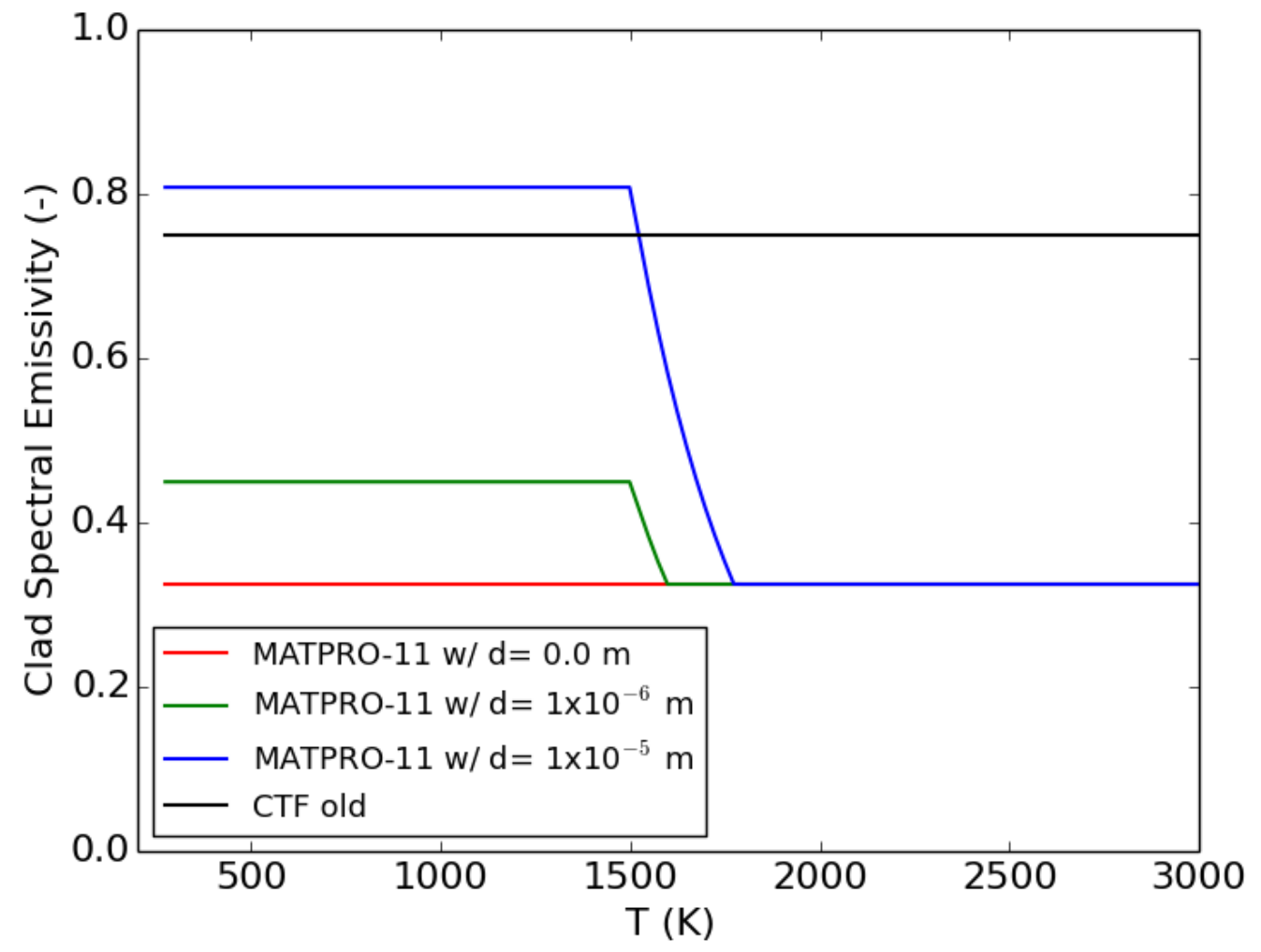

Figure 9.15: Zirconium cladding emissivity (Equation 9.23). 


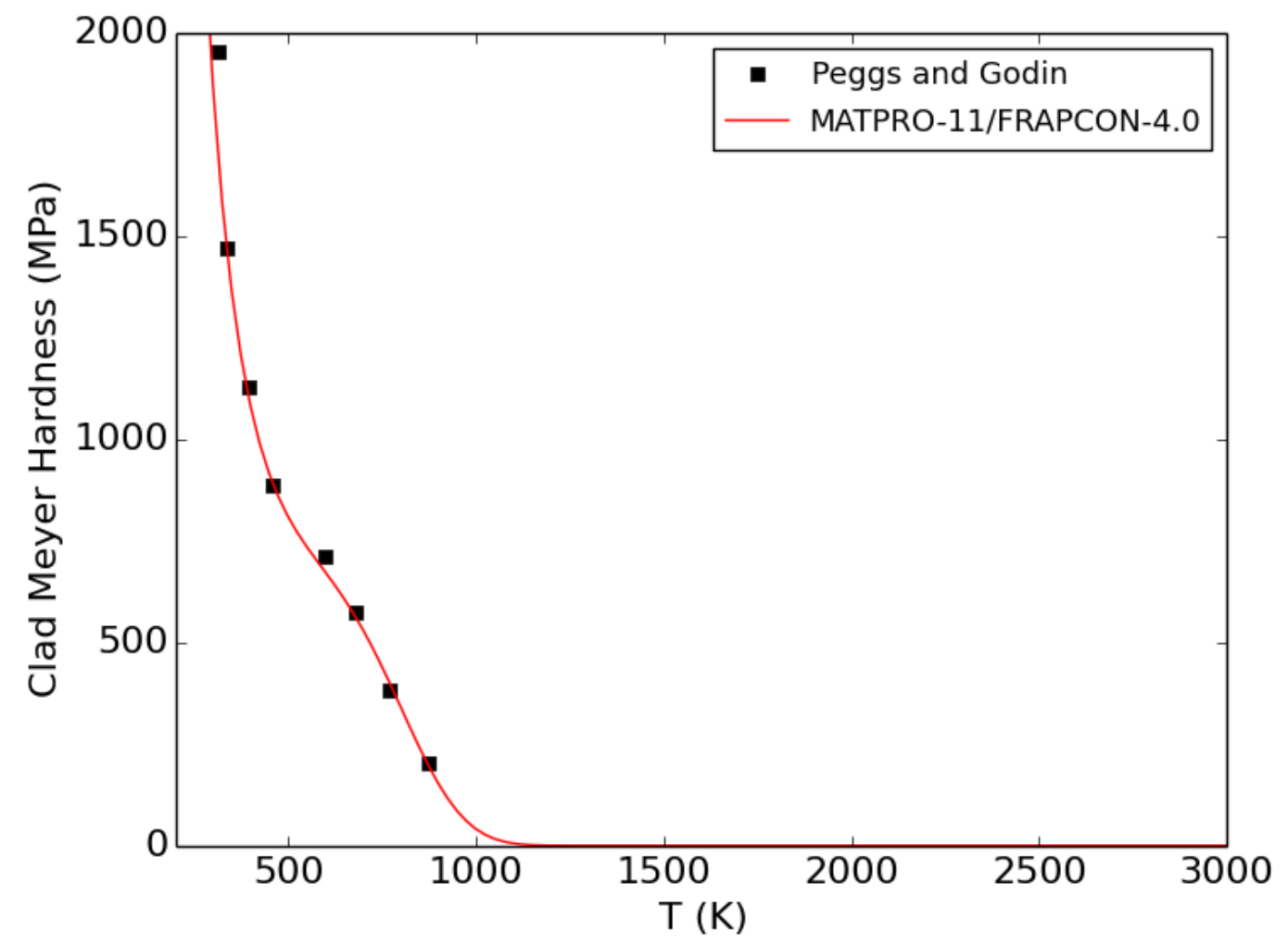

Figure 9.16: Meyer hardness of cladding. 
and for the $\beta$-phase with temperatures between $1273^{\circ} \mathrm{K}$ and the melting point:

$$
\begin{aligned}
\epsilon_{\mathrm{Z}, \text { axial }} & =9.7 \cdot 10^{-6} T-1.1 \cdot 10^{-2} \\
\epsilon_{\mathrm{Z} \text {,radial }} & =9.7 \cdot 10^{-6} T-9.45 \cdot 10^{-3}
\end{aligned}
$$

where: $T=$ temperature $\left({ }^{\circ} \mathrm{K}\right)$

Very little data are available for the $\alpha$-phase to $\beta$-phase transition zone, which exists for temperatures from $1073^{\circ} \mathrm{K}$ to $1273^{\circ} \mathrm{K}$. A lookup table was entered into CTF for this range, the data being taken from Scott's data[129] as presented in MATPRO-11 (Revision 1)[62]. Axial and radial strain data for Zircaloy are given in Table 15. Figure 9.17 shows the cladding axial and radial thermal expansion as a function of temperature.

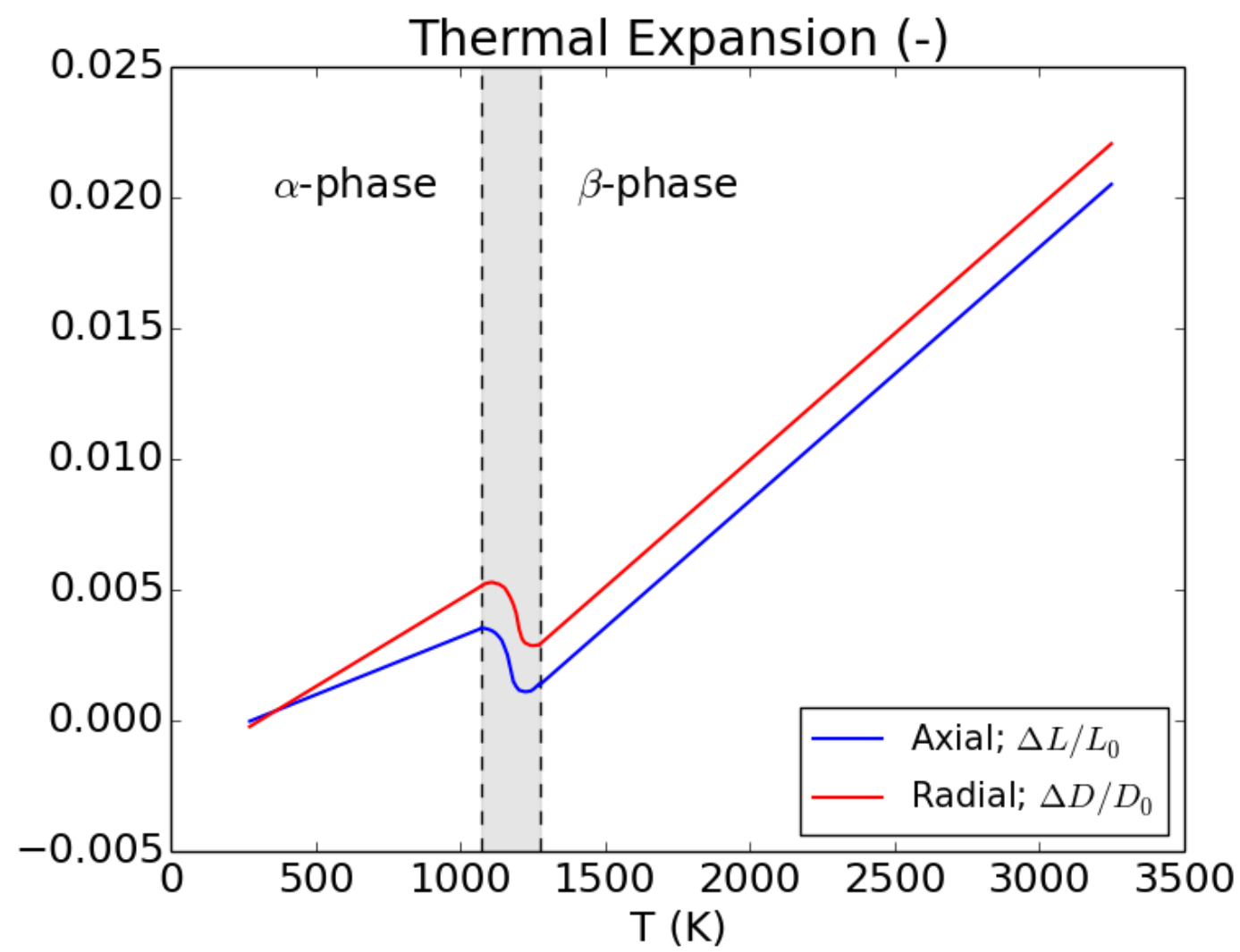

Figure 9.17: Cladding axial and radial thermal expansion.

\subsubsection{Cladding Elastic Modulus and Shear Modulus}

Stress in the cladding is calculated using Young's modulus. The shear modulus for Zircaloy is calculated as in the CELMOD and CSHEAR subroutines of MATPRO-11. Effects are oxidation $\left(K_{1}\right)$, cold work $\left(K_{2}\right)$, and fast neutron fluence $\left(K_{3}\right)$ are neglected. 
Table 15: Zircaloy axial strain data for the $\alpha$-phase to $\beta$-phase transition zone

\begin{tabular}{ccc}
\hline$T\left({ }^{\circ} \mathrm{K}\right)$ & $\epsilon_{Z, \text { axial }}(\mathrm{ft} / \mathrm{ft}) \cdot 10^{3}$ & $\epsilon_{Z, \text { radial }}(\mathrm{ft} / \mathrm{ft}) \cdot 10^{3}$ \\
\hline 1073.15 & 3.52774 & 5.1395 \\
1083.15 & 3.53 & 5.22 \\
1093.15 & 3.50 & 5.25 \\
1103.95 & 3.46 & 5.28 \\
1113.15 & 3.41 & 5.28 \\
1123.15 & 3.33 & 5.24 \\
1133.15 & 3.21 & 5.22 \\
1143.15 & 3.07 & 5.15 \\
1153.15 & 2.80 & 5.08 \\
1163.15 & 2.50 & 4.90 \\
1173.15 & 2.00 & 4.70 \\
1183.15 & 1.50 & 4.45 \\
1193.15 & 1.30 & 4.10 \\
1203.15 & 1.16 & 3.50 \\
1213.15 & 1.13 & 3.13 \\
1223.15 & 1.10 & 2.97 \\
1233.15 & 1.11 & 2.92 \\
1243.15 & 1.13 & 2.87 \\
1253.15 & 1.20 & 2.86 \\
1263.15 & 1.30 & 2.88 \\
1273.15 & 1.40 & 2.90 \\
\hline
\end{tabular}


For the $\alpha$-phase wtih $T<1093^{\circ} \mathrm{K}$ :

$$
\begin{aligned}
& E_{Z}=1.088 \cdot 10^{11}-5.475 \cdot 10^{7} T \\
& G_{Z}=4.04 \cdot 10^{10}-2.168 \cdot 10^{7} T
\end{aligned}
$$

and for the $\beta$-phase with $T>1239^{\circ} \mathrm{K}$ :

$$
\begin{aligned}
& E_{Z}=9.21 \cdot 10^{10}-4.05 \cdot 10^{7} \mathrm{~T} \\
& G_{Z}=3.48 \cdot 10^{10}-1.66 \cdot 10^{7} \mathrm{~T}
\end{aligned}
$$

where: $T=$ temperature $\left({ }^{\circ} \mathrm{K}\right)$

$E_{Z}=$ Young's modulus $(\mathrm{Pa})$

$G_{Z}=$ shear modulus $(\mathrm{Pa})$

Linear interpolation is used for calculating $E_{Z}$ and $G_{Z}$ in the $\alpha$-phase to $\beta$-phase transition region. Poisson's ratio is obtained as follows:

$$
\nu=\frac{E}{2 G}-1
$$

where: $\nu=$ Poissons's ratio (unitless)

$E=$ Young's modulus $(\mathrm{Pa})$

$G=$ shear modulus $(\mathrm{Pa})$

Figure 9.18 shows the cladding elastic moduli for the isotropic material as a function of temperature.

\subsection{Gas Properties}

CTF includes capabilities of modeling several non-condensable gases in the simulation, including: air, argon, helium, hydrogen, krypton, nitrogen, oxygen, and xenon. This necessitates including means of calculating gas properties for different system conditions. Non-condensable gases are modeled by the user providing information on the gases present in the system, their concentrations, and the reference enthalpy of the gas mixture. CTF includes specific heat and gas constant data for the gases to enable the calculation of gas mixture enthalpy and density.

\subsubsection{Gas Conductivity}

Properties are available for 6 different types of nuclear fuel rod fill gases. The gas conductivity as well as the gas molecular weight $\left(\mathrm{M}_{i}\right)$ is needed for determination of the mixture gas conductivity. The fill gas conductivity (BTU/hr-ft-F) is given by

$$
K=a * T_{\circ}^{b}
$$

where: $K=$ thermal conductivity (BTU/hr-ft-F)

$a, b=$ the empirical coefficients

$T=$ temperature $\left({ }^{\circ} \mathrm{R}\right)$ 


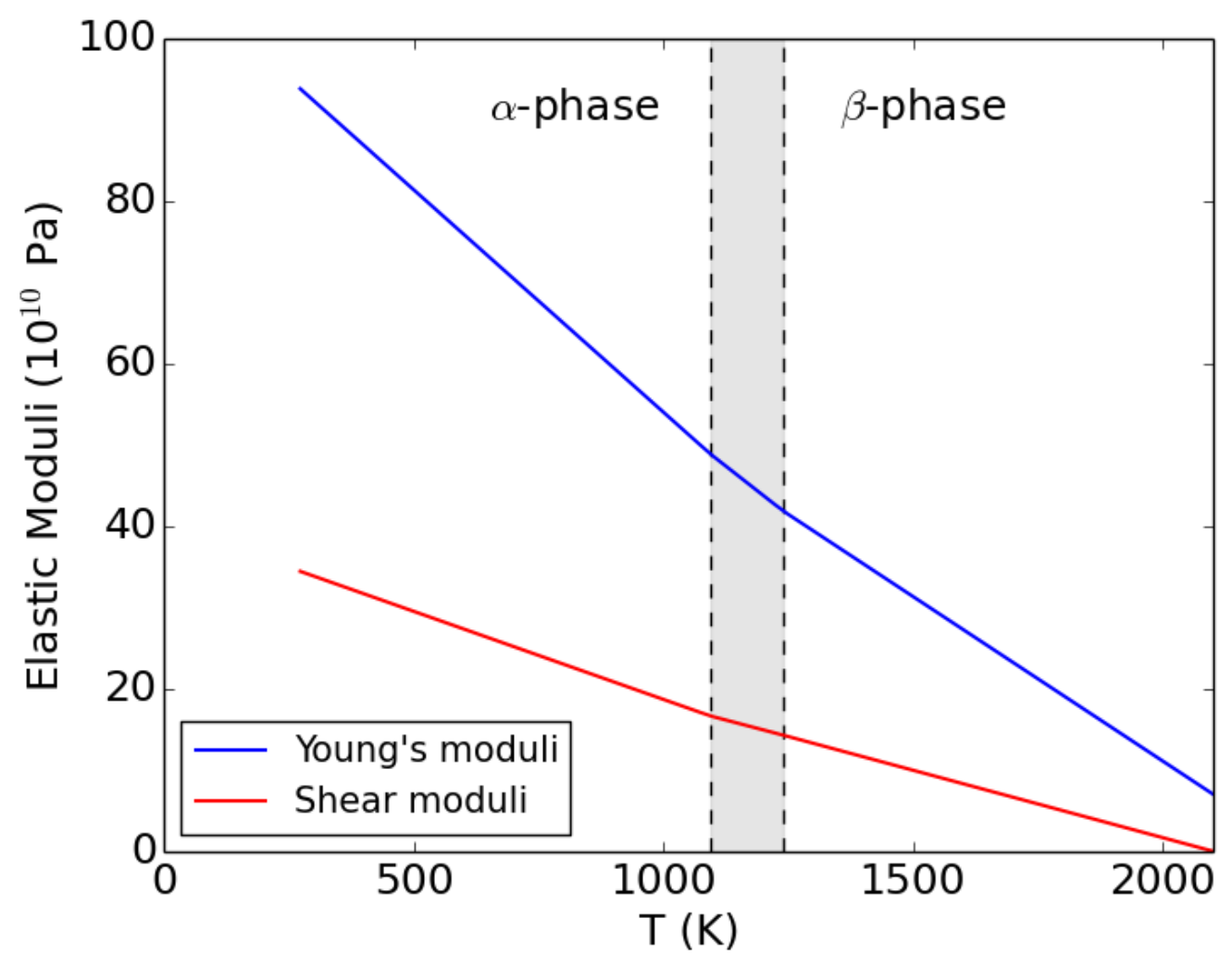

Figure 9.18: Cladding elastic moduli for isotropic material.

Table 16: Fill gas conductivity relations

\begin{tabular}{ccccc}
\hline & Gas & $\mathbf{a}$ & $\mathbf{b}$ & $\mathbf{M}_{i}$ \\
\hline 1 & Helium & $1.314 \cdot 10^{-3}$ & 0.668 & 4.003 \\
2 & Xenon & $1.395 \cdot 10^{-5}$ & 0.872 & 131.3 \\
3 & Argon & $1.31 \cdot 10^{-3}$ & 0.701 & 39.944 \\
4 & Krypton & $1.588 \cdot 10^{-5}$ & 0.92331 & 83.8 \\
5 & Hydrogen & $5.834 \cdot 10^{-4}$ & 0.8213 & 2.016 \\
6 & Nitrogen & $7.35 \cdot 10^{-5}$ & 0.846 & 28.8 \\
\hline
\end{tabular}


The temperature dependent conductivities and gas molecular weights are given in Table 16.

Figure 9.19 shows the fill gas thermal conductivity as a function of temperature for helium, xenon, argon, krypton, hydrogen and nitrogen.

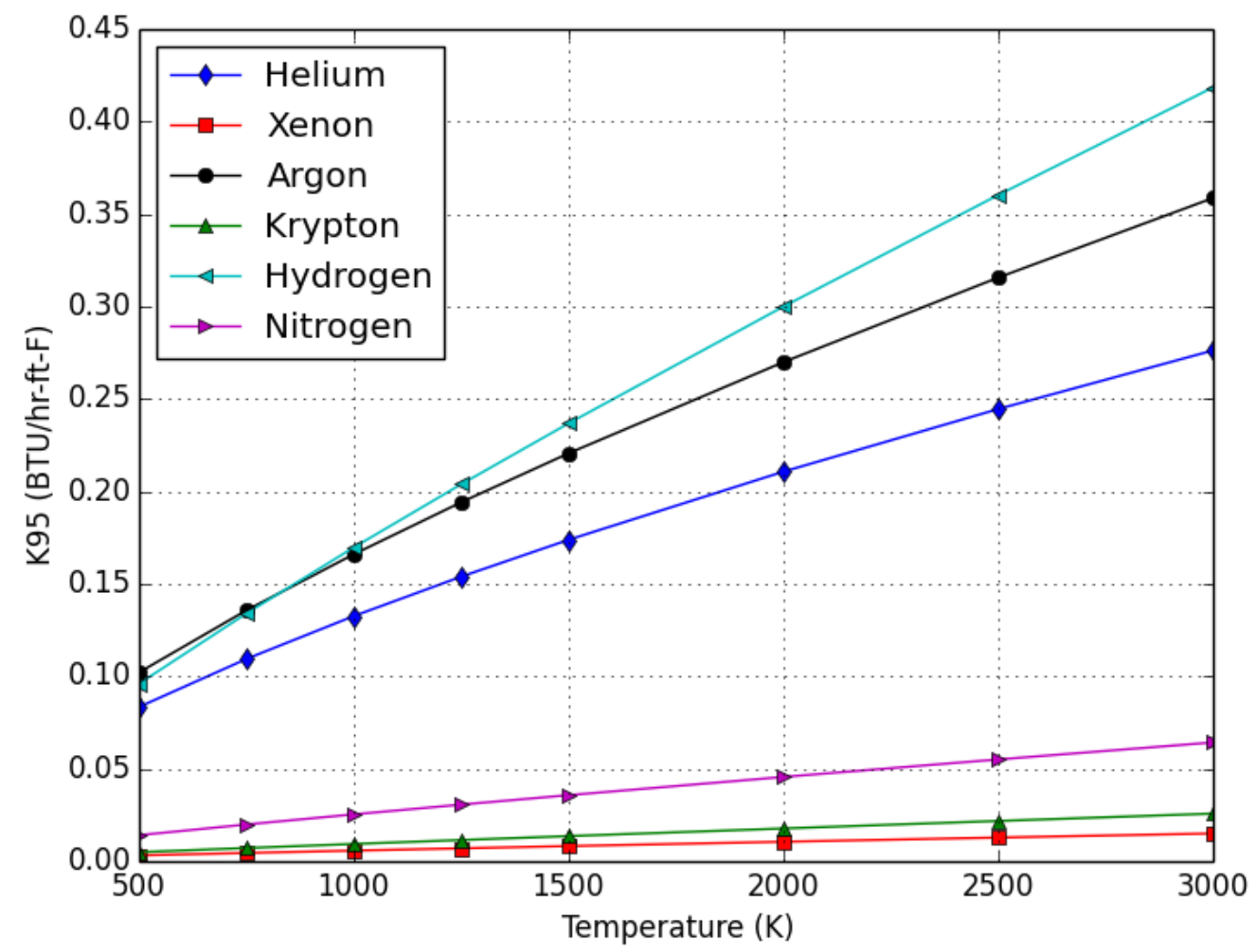

Figure 9.19: Fill gas thermal conductivity.

\subsubsection{Molecular Weight}

The molecular weight of the gases supported by CTF are given in Table 17.

\subsubsection{Gas Specific Heat}

Specific heat is determined in the GASP subroutine. The specific heat for the gas mixture is calculated by summing the specific heats of individual constituent gases times their mass fraction. 
Table 17: Molecular weights and specific gas constants of gases used in CTF

\begin{tabular}{lcc}
\hline Gas & $\begin{array}{c}\text { Molecular } \\
\text { Weight }\end{array}$ & $\begin{array}{c}\text { Specific Gas Constant } \\
\left(\mathrm{ps} /{ }^{\circ} \mathrm{R}\left(\mathrm{lbm} / \mathrm{ft}^{3}\right)\right.\end{array}$ \\
\hline Air & 28.96 & 53.34 \\
Argon & 39.94 & 38.66 \\
Helium & 4.003 & 386.0 \\
Hydrogen & 2.016 & 766.4 \\
Krypton & 83.70 & 18.459 \\
Nitrogen & 28.016 & 55.15 \\
Oxygen & 32.0 & 48.28 \\
Xenon & 131.3 & 11.767 \\
\hline
\end{tabular}

$$
C p=\Sigma_{i=1}^{n g a s} m f_{i} C p_{i}
$$

where: $m f=$ the mass fraction of the gas

The mass fraction of the gas is found by dividing the gas molecular weight by the total molecular weight of the gas mixture.

$$
m f_{i}=\frac{\alpha_{i} m w_{i}}{\sum_{i=1}^{n g a s} \alpha_{i} m w_{i}}
$$

where: $\alpha_{i}=$ the fraction of the individual gas in the mixture

$m w_{i}=$ the molecular weight of the gas

The specific heats of the individual gases are found by correlation. For air, the correlation to be used depends on the gas temperature. Note that the gas mixture temperature is considered to be in thermoequilibrium with the vapor temperature and, thus, they are equal in CTF. The air specific heat, in units of $\mathrm{BTU} / \mathrm{lbm}^{\circ} \mathrm{F}$, is:

$$
\begin{aligned}
& C p_{\text {air }}=0.244388-4.20419 \cdot 10^{-5} T+9.61128 \cdot 10^{-8} T^{2}-1.16383 \cdot 10^{-11} T^{3}, \text { for } T<=600^{\circ} \mathrm{K}(9.34 \mathrm{a}) \\
& C p_{\text {air }}=0.208831+7.71027 \cdot 10^{-5} T-8.56726 \cdot 10^{-9} T^{2}-4.75772 \cdot 10^{-12} T^{3}, \text { for } T>600^{\circ} \mathrm{K} \quad(9.34 \mathrm{~b})
\end{aligned}
$$

Argon specific heat is set to a constant of $0.12428 \mathrm{BTU} / \mathrm{lbm}^{\circ} \mathrm{F}$. Helium specific heat is set to a constant of $1.2404 \mathrm{BTU} / \mathrm{lbm}^{\circ} \mathrm{F}$. The hydrogen specific heat is temperature dependent.

$$
\begin{aligned}
& C p_{\mathrm{H}}=1.46910+1.60057 \cdot 10^{-2} T-4.44048 \cdot 10^{-5} T^{2}+4.21220 \cdot 10^{-8} T^{3}, \text { for } T<=400^{\circ} \mathrm{K} \\
& C p_{\mathrm{H}}=3.56903-4.8959 \cdot 10^{-4} T+6.22549 \cdot 10^{-6} T^{2}-1.19686 \cdot 10^{-10} T^{3}, \text { for } T>400^{\circ} \mathrm{K}
\end{aligned}
$$


The krypton specific heat is set to $0 \mathrm{BTU} / \mathrm{lbm}^{\circ} \mathrm{F}$. Nitrogen specific heat is temperature dependent.

$$
\begin{aligned}
& C p_{N}=0.259934-8.42119 \cdot 10^{-5} T+1.72117 \cdot 10^{-7} T^{2}-6.7294 \cdot 10^{-11} T^{3}, \text { for } T<=775^{\circ} \mathrm{K} \\
& C p_{N}=0.201678+1.08013 \cdot 10^{-4} T-3.32212 \cdot 10^{-8} T^{2}+2.45228 \cdot 10^{-12} T^{3}, \text { for } T>775^{\circ} \mathrm{K}
\end{aligned}
$$

The oxygen specific heat is temperature dependent.

$$
\begin{aligned}
& C p_{O}=0.222081-7.6923 \cdot 10^{-5} T+2.78765 \cdot 10^{-7} T^{2}-1.70107 \cdot 10^{-10} T^{3}, \text { for } T<=760{ }^{\circ} \mathrm{K} \\
& C p_{O}=0.1771+1.49509 \cdot 10^{-4}-8.4494 \cdot 10^{-8} T^{2}+1.83236 \cdot 10^{-11} T^{3}, \text { for } T>760^{\circ} \mathrm{K}
\end{aligned}
$$

The xenon specific heat is set to $0 \mathrm{BTU} / \mathrm{lbm}^{\circ} \mathrm{F}$.

Figure 9.20 shows the specific heat of air, nitrogen, hydrogen and oxygen as a function of temperature.

\subsubsection{Gas Density}

The gas mixture density is found using the ideal gas law.

$$
\rho_{\text {mix }}=\frac{P}{R_{\text {mix }}(T+459.6)}
$$

where: $T=$ the temperature $\left({ }^{\circ} \mathrm{F}\right)$

$R=$ the gas constant $\left(\mathrm{psi} /{ }^{\circ} \mathrm{R}\left(\mathrm{lbm} / \mathrm{ft}^{3}\right)\right)$

The gas constant for a mixture is calculated by summing the products of individual gas mass fractions and their individual gas constants.

$$
R_{\text {mix }}=\Sigma_{i=1}^{n g a s} m f_{i} R_{i}
$$

The individual specific gas constants were given in Table 17. Units were in pounds per square foot (psf) in the table, so those values are converted to psi before being used to calculate density. Gas mixture density is calculated in various locations of the source code as it is needed.

\subsubsection{Gas Enthalpy}

The enthalpy of the gas mixture is calculated using the specific heat of the mixture. The temperature change from the reference temperature, $T_{\text {ref }}$, is multiplied by $C p$ and added to the reference enthalpy, $h_{\text {ref }}$. 

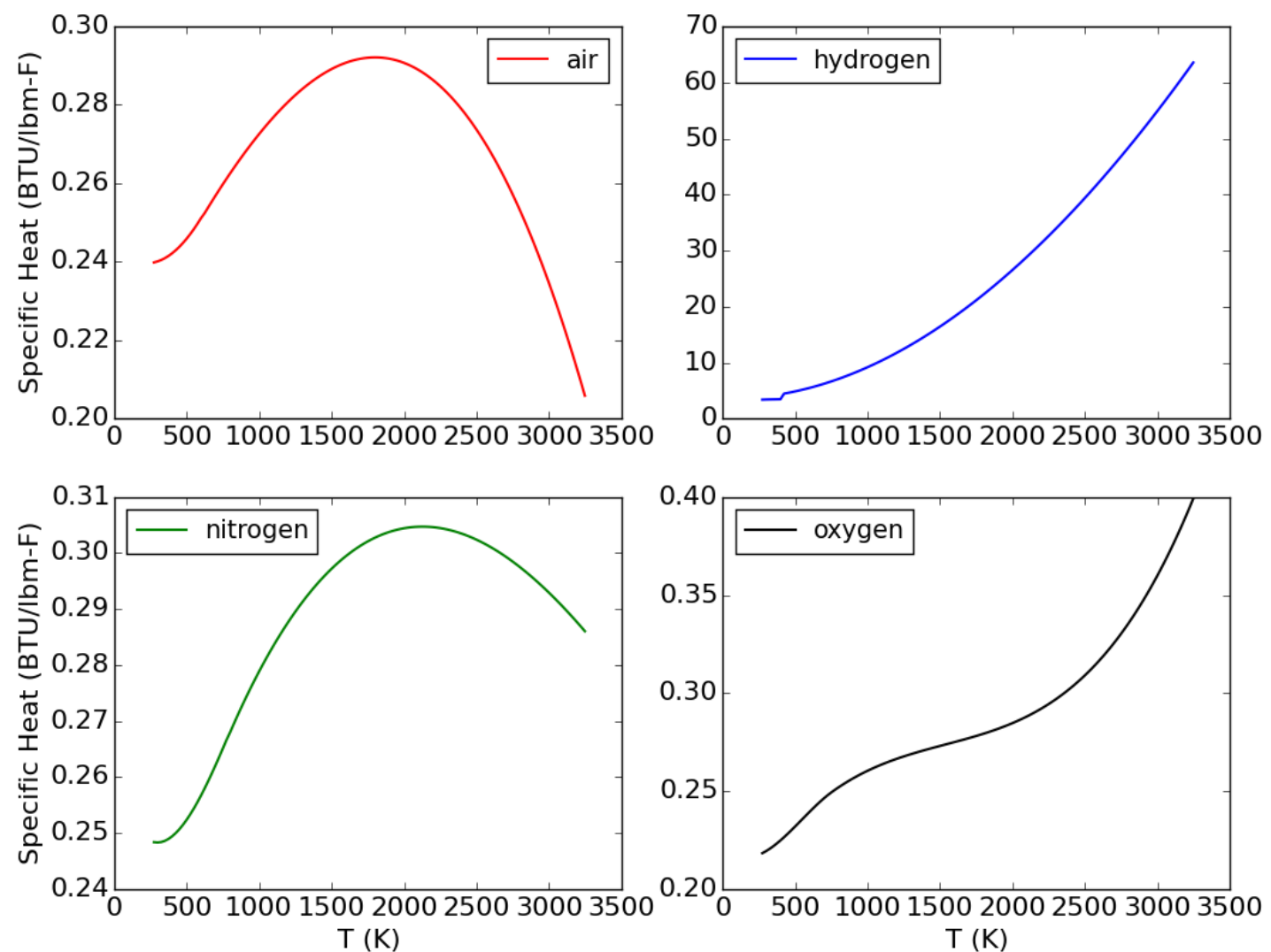

Figure 9.20: Specific heat of air, nitrogen, hydrogen and oxygen. 


$$
H_{\text {mix }}=C p_{\operatorname{mix}}\left(T_{v}-T_{v}^{r e f}\right)+H_{\text {ref }}
$$

If the solution is in the middle of a transient, the previous time step temperature and enthalpy is used in place of the reference values. 


\section{Bibliography}

[1] M.J. Thurgood et al. "COBRA-TF Development". In: 8th Water Reactor Safety Information Meeting. 1980.

[2] M.N. Avramova. CTF-A Thermal-Hydraulic Subchannel Code for LWR Transient Analyses: Theory Manual: Volume II Linearization of Mass and Energy Equations. Tech. rep. Pennsylvania State University, 2009.

[3] S.V. Patankar. Numerical Heat Transfer and Fluid Flow. McGraw-Hill, 1980.

[4] M.N. Avramova. CTF-A Thermal-Hydraulic Subchannel Code for LWR Transient Analyses: Theory Manual: Volume I Momentum Equations. Tech. rep. Pennsylvania State University, 2009.

[5] M.N. Avramova. CTF: A Thermal Hydraulic Sub-Channel Code for LWR Transient Analyses, User's Manual. Feb. 2009.

[6] E. Ozdemir. "Multidimensional Boron Transport Modeling in a Subchannel Approach". PhD thesis. The Pennsylvania State University, 2012.

[7] S. Kliem et al. "Experiments at the mixing test facility ROCOM for benchmarking of CFD codes". In: Nuclear Engineering and Design 238 (2008), pp. 566-576.

[8] J. Tuunanen et al. "Long-term emergency cooling experiments with aqueous boric acid solution with the REWET-II and VEERA facilities." In: Proc. Int. ENS/ANS Conf. On Thermal Reactor Safety. 1988.

[9] J. Tuunanen, H. Tuomisito, and P. Raussi. "Experimental and analytical studies of boric acid concentrations in a VVER-440 reactor during long-term cooling period of loss-of-coolant accidents". In: Nuclear Engineering and Design 148 (1994), pp. 217-231.

[10] J. Freixa, R. Francesc, and C. Pretel. "Boron Transport Model with Physical Diffusion for RELAP5". In: Nuclear Technology 160 (2007), pp. 205-215.

[11] E. Ozdemir, M. N. Avramova, and K. Sato. "Multi-dimensional boron transport modeling in subchannel approach: Part I. Model selection, implementation and verification of COBRA-TF boron tracking model". In: Nuclear 278 (2014), pp. 701-712.

[12] E. Ozdemir and M. N. Avramova. "Multi-dimensional boron transport modeling in subchannel approach: Part II. Validation of CTF boron tracking model and adding boron precipitation model". In: Nuclear E 278 (2014), pp. 713-722.

[13] P. L. Roe. "Some Contributions to the Modeling of Discontinuous Flow". In: Lectures of Applied Mathematics. Vol. 22. 163. 1975. 
[14] H. C. Yee and R. F. Warming. "Implicit Total Variation Dismishing (TVD) Schemes for Steady-State Calculations". In: Computational Physics 57 (1985), p. 327.

[15] A. Einstein. "On the Movement of Small Particles Suspended in Stationary Liquids Required by the Molecular -Kinetic Theory of Heat". In: Annals of Physics 17 (1905), p. 549.

[16] P. Zanetti. "Pollution Modelling: Theories, Computational Methods and Available Software". In: Computational Mechanics Publications, Southhamption, United Kingdom (1990).

[17] Numerical diffusion and Part 1. One-dimensional flows the tracking of solute fields in system codes. "R. Macian-Juan and J. Mahaffy". In: Nuclear Engineering and Design 179 (1998), pp. 297-319.

[18] S. Y. Kim et al. "High temperature boric acid solubility during post-LOCA long-term cooling period". In: Transactions of the American Nuclear Society 99 (2008), pp. 489-490.

[19] P. Griffith and G.B. Wallis. "Two-Phase Slug Flow". In: J. Heat Transfer 83 (1961), pp. 307-320.

[20] P.A. Griffith and G.A. Snyder. The Bubbly-Slug Transition in High Velocity Two-Phase Flow. Tech. rep. TID-20947. MIT, July 1964.

[21] K. Mishima and M. Ishii. "Theoretical Prediction on Onset of Horizontal Slug Flow". In: J. Fluids Eng. 102 (1980), pp. 441-445.

[22] N.A. Radovich and R. Moissis. The Transition from Two-Phase Bubble Flow to Slug Flow. Tech. rep. 7-7673-22. MIT, 1962.

[23] Y. Taitel, D. Bornea, and A.E. Dukler. "Modeling flow pattern transitions for steady upward gas-liquid flow in vertical tubes". In: AIChE Journal 26.3 (1980), pp. 345-354.

[24] M. Ishii and K. Mishima. Study of Two-Fluid Model and Interfacial Area. Tech. rep. NUREG/CR1873, ANL-80-111. Argonne National Laboratory, Dec. 1980.

[25] G.B. Wallis. One-Dimensional Two-Phase Flow. McGraw-Hill, 1969.

[26] W. H. McAdams. Heat Transmission. First. McGraw-Hill, New York, 1933.

[27] D. J. Zigrang and N. D. Sylvester. "Explicit Approximations to the Solution of Colebrook's Friction Factor Equation". In: AICHE J 28.3 (May 1982), pp. 514-515.

[28] S. W. Churchill. "Friction Factor Equation Spans All Fluid-Flow Regimes". In: Chemical Engineering 7 (Nov. 1977), pp. 94-95.

[29] N.E. Todreas and M.S. Kazimi. Nuclear Systems II Elements of Thermal Hydraulic Design. Hemisphere Publishing Corporation, 1990.

[30] R.T. Lahey and F.J. Moody. The Thermal-Hydraulics of a Boiling Water Nuclear Reactor. Tech. rep. ANS Monograph, 1977.

[31] J.T. Rogers and R.G. Rosehart. "Mixing by Turbulent Interchange in Fuel Bundles. Correlations and Influences". In: ASME. Paper 75-HT-31. 1972, pp. 1-12.

[32] S.G. Beus. A Two-Phase Turbulent Mixing Model for Flow in Rod Bundles. Tech. rep. WAPD-TM2438. Bettis Atomic Power Laboratory, 1971.

[33] A. Faya, L. Wolf, and N.E. Todreas. Canal Users Manual. MIT-EL-79-028. 1979.

[34] M. Ishii. One-Dimensional Drift-Flux Model and Constitutive Equations for Relative Motion between Phases in Various Two-Phase Flow Regimes. Tech. rep. ANL-77-47. Argonne National Laboratory, Sept. 1977.

[35] M. Ishii and T.C. Chawla. Local Drag Laws in Dispersed Two-Phase Flow. Tech. rep. NUREG/CR1230. Argonne National Laboratory, Dec. 1979. 
[36] W.H. Henstock and T.J. Hanratty. "The Interfacial Drag and the Height of the Wall Layer in Annular Flows". In: AIChE Journal 22.6 (1976), pp. 990-1000.

[37] A.E. Dukler et al. Two-Phase Interactions in Countercurrent Flow Studies of the Flooding Mechanism. Tech. rep. NUREG/CR-0617. Nuclear Regulatory Commission, 1979.

[38] P.N. Rowe, K.T. Claxton, and J.B. Lewis. "Heat and Mass Transfer from a Single Sphere in an Extensive Flowing Fluid". In: Trans. Instn. Chem. Engrs. Vol. 43. 1965, T14-T31.

[39] M. J. Boussinesq. "Calcul du Pouvoir Refroidissant des Courants Fluids (Calculation of the Cooling power of a Fluid Stream)". In: Journal de Mathématiques Pures et Appliquées 1 (1905), p. 310.

[40] K. Lee and D.J. Ryley. "The Evaporation of Water Droplets in Superheated Steam". In: Trans. ASME J. Heat Transfer 90 (1968), pp. 445-451.

[41] N. Frössling. "The Evaporation of Falling Drops". In: Gerlands Beitrage zur Geophysik 52 (1938), pp. 107-216.

[42] M.C. Yuen and L.W. Chen. "Heat-Transfer Measurements of Evaporating Liquid Droplets". In: Int $J$ Heat and Mass Transfer 21 (1978), pp. 537-542.

[43] J. G. M. Andersen. REMI/HEAT COOL, A Model for Evaluation of Core Heat-Up and Emergency Core Spray Cooling System Performance for Light-Water-Cooled Nuclear Power Reactors. Tech. rep. 296. RISO National Laboratory, Sept. 1973.

[44] A.P. Colburn. "A Method of Correlating Forced Convection Heat Transfer Data and a Comparison with Fluid Friction". In: Trans Am Inst Chem Eng. Vol. 29. 1933, pp. 174-210.

[45] M. Ishii and M.A. Grolmes. "Inception Criteria for Droplet Entrainment in Two-Phase Concurrent Film Flow". In: AIChE Journal 21.2 (1975), p. 308.

[46] G.A. Hughmark. "Film Thickness Entrainment and Pressure Drop in Upward Annular and Dispersed Flow". In: AICHE J 19 (1973), pp. 1062-1065.

[47] K. Ha, J. Jeong, and S. Sim. "Improvement of Liquid Droplet Entrainment Model in the COBRA-TF Code". In: Journal of the Korean Nuclear Society 30.3 (1998), pp. 181-193.

[48] P.B. Whalley. Experimental Wave and Entrainment Measurements in Vertical Annular Two-Phase Flow. Tech. rep. AERE-R7521. Atomic Energy Research Establishment, 1973.

[49] J. Wurtz. An Experimental and Theoretical Investigation of Annular Steam-Water flow in Tubes and Annuli at 30 to 90 Bar. Tech. rep. 372. RISO National Laboratory, Denmark, 1978.

[50] I.I. Paleev and B.A. Filippovich. "Phenomena of Liquid Transfer in Two-Phase Dispersed Annular Flow". In: International Journal of Heat and Mass Transfer 9 (1966), pp. 1089-1093.

[51] L.B. Cousins. "Liquid Mass Transfer in Annular Two-Phase Flow". In: Symposium on Two-Phase Flow, Volume 2. 1965, pp. 401-430.

[52] P.B. Whalley. "The Calculation of Dryout in a Rod Bundle". In: International Journal of Multi-phase Flow 13 (1977), pp. 501-515.

[53] I. Kataoka. "Generation and Size Distribution of Droplet in Annular Two-Phase Flow". In: Journal of Fluids Engineering 105 (1983).

[54] J.E. Adams and A.J. Clare. A Preliminary Study of Droplet Breakup at PWR Spacer Grids. Tech. rep. Central Electric Generating Board, PWR/HTWS/P, 1983.

[55] S.L. Lee et al. Reentrainment of Droplets from Grid Spacers in Mist Flow Portion of a LOCA Reflood of a PWR. Tech. rep. NUREG-CR-0043. Nuclear Regulatory Commisson, 1982. 
[56] S.C. Yao et al. "Dynamics of Droplets Impacting on Thin Heated Strips". In: National Heat Transfer Conference. 1985.

[57] L.H.J. Wachters and N.A.J. Westerling. "The Heat Transfer from a Hot Wall to Impinging Water Drops in a Spheroidal State". In: Chem. Eng. Sci. 21 (1966), pp. 1047-1056.

[58] L.H.J. Wachters et al. "The Heat Transfer from a Hot Wall to Impinging Mist Droplets in the Spheroidal State". In: Chem. Eng. Sci. 21 (1966), pp. 1231-1238.

[59] K. Takeuchi et al. "Experimental Studies on the Behavior of a Small Droplet Impinging upon a Hot Surface". In: Proceedings of the Second International Conference on Liquid Atomization and Spray Systems. 1982, pp. 397-404.

[60] D.S. Trent and J.R. Welty. A Summary of Numerical Methods for Solving Transient Heat Conduction Problems. Tech. rep. Bulletin No. 49. Engineering Experimental Station, Oregon State University, 1974.

[61] G.E. Kendall and W.M. Rohsenow. Heat Transfer to Impacting Drops and Post Critical Heat Flux Dispersed Flow. Tech. rep. 85694-100. MIT, 1978.

[62] D.L. Hagrman, G.A. Reymann, and R.E. Manson. MATPRO-Version 11: A Handbook of Material Properties for Use in the Analysis of Light Water Reactor Fuel Rod Behavior. Tech. rep. NUREG/CR0497. U.S. Nuclear Regulatory Commission, 1980.

[63] G.A. Berna et al. FRAPCON-2: A Computer Code for the Calculation of Steady-State ThermalMechanical Behavior of Oxide Fuel Rods. Tech. rep. NUREG/CR-1845. U.S. Nuclear Regulatory Commission, 1981.

[64] D.R. Coleman and E.T. Laats. FRAP-T3, A Computer Code for the Transient Analysis of Oxide Fuel Rods-Model Assessment Report. Tech. rep. NUREG/CR-0555, TREE-1320. U.S. Nuclear Regulatory Commission, 1979.

[65] D.D. Lanning and C.R. Hann. Review of Methods Applicable to the Calculation of Gap Conductance in Zircaloy-Clad $\mathrm{UO}_{2}$ Fuel Rods. Tech. rep. BNWL-1894. Pacific Northwest Laboratory, 1975.

[66] W.R. Lloyd et al. "Heat Transfer in Multicomponent Monatomic Gases in the Low, Intermediate, and High Pressure Regime". In: Nuclear Thermionics Conference. 1973.

[67] A. Ullman, R. Acharya, and D.R. Olander. "Thermal Accommodation Coefficients of Inert Gases on Stainless Steel and $\mathrm{UO}_{2} "$. In: Journal of Nuclear Materials 51 (1974), pp. 277-279.

[68] L.B. Thomas. Fundamentals of Gas-Surfacing Interactions. Academic Press, New York, 1967.

[69] M.G. Cooper, B.B. Mikic, and M.M. Yavonovich. "Thermal Contact Conductance". In: Journal of Heat and Mass Transfer 12 (1969).

[70] N. Todreas and G. Jacobs. "Thermal Contact Conductance of Reactor Fuel Elements". In: Nuclear Science and Engineering 50 (1973), p. 283.

[71] J.V. Cathcart et al. Zirconium Metal-Water Oxidation Kinetics IV, Reaction Rate Studies. Tech. rep. ORNL/NUREG-17, 1977.

[72] N. Zuber, M. Tribus, and J.W. Westwater. "The Hydrodynamic Crisis in Pool Boiling of Saturated and Subcooled Liquids". In: International Development in Heat Transfer: Proceedings of 1961-62 International Heat Transfer Conference. 1961, pp. 230-236.

[73] T.A. Bjornard and P. Griffith. "PWR Blowdown Heat Transfer". In: Thermal and Hydraulic Aspects of Nuclear Reactor Safety. Vol. 1. ASME. 1977, pp. 17-41.

[74] J.H. Lienhard and V.K. Dhir. "Hydrodynamic Prediction of Peak Pool-Boiling Heat Fluxes from Finite Bodies". In: J. Heat Transfer 95.2 (1973), pp. 152-158. 
[75] L. Sardh and K.M. Becker. Assessments of CHF Correlations Based on Full-Scale Rod Bundle Experiments. Tech. rep. KTH-NEL-36. Royal Institute of Technology (KTH), 1986.

[76] L. Biasi et al. "Studies on Burnout, Part 3". In: Energia Nucleare 14.9 (1967), pp. 530-536.

[77] L.S. Tong. "Prediction of Departure from Nucleate Boiling for an Axially Non-Uniform Heat Flux Distribution". In: Journal of Nuclear Energy 21 (1967), pp. 241-248.

[78] L.S. Tong. Boiling Crisis and Critical Heat Flux. Tech. rep. TID-25887. US Atomic Energy Commission, 1972.

[79] N.E. Todreas and M.S. Kazimi. Nuclear Systems I: Thermal Hydraulic Fundamentals. Hemisphere Publishing Corporation, 1993.

[80] R.W. Bowring. A Simple but Accurate Round Tube, Uniform Heat Flux Dryout Correlation over the Pressure Range 0.7 to 17 MPa. Tech. rep. AEE Winfrith, 1972.

[81] D.C. Groeneveld et al. The 2006 CHF look-up table. Tech. rep. Nuclear Engineering and Design, 2007.

[82] R. Salko et al. CTF Validation and Verification. The Pennsylvania State University. 2015.

[83] D.C. Groeneveld et al. Look-Up Tables for Predicting CHF and Film Boiling Heat Transfer - Past, Present and Future (NURETH). Tech. rep. Chalk River Lab, AECL, 2003.

[84] Tae-Hyun Chun et al. Assessment of a tube-based bundle chf prediction method using a subchannel code. Tech. rep. KAERI, 1998.

[85] S.C. Yao, L.E. Hochreiter, and W.J. Leech. "Heat-Transfer Augmentation in Rod Bundles Near Grid Spacers". In: Journal of Heat Transfer 104 (1982), pp. 76-81.

[86] J.C. Chen. "A Correlation for Boiling Heat Transfer to Saturated Fluids in Convective Flow". In: ASME 63-HT-34. 1963.

[87] J.R.S. Thom et al. "Boiling in Subcooled Water During Flow Up Heated Tubes for Annuli". In: Symposium on Boiling Heat Transfer in Steam Generating Units and Heat Exchangers, Manchester, London. 1965.

[88] R.E. Henry. "A Correlation for the Minimum Film Boiling Temperature". In: AICHE Symposium Series. Vol. 138. 1974, pp. 81-90.

[89] F.W. Dittus and L.M.K. Boelter. "Heat Transfer in Automobile Radiators fo the Tubular Type". In: Publications in Engineering 2. University of California, Berkeley, 1930, pp. 443-461.

[90] S. Wong and L.E. Hochreiter. Analysis of the FLECHT-SEASET Unblocked Bundle Steam Cooling and Boiloff Tests. Tech. rep. NRC/EPRI/Westinghouse, 1981.

[91] E.M. Sparrow et al. "Heat Transfer to Longitudinal Laminar Flow Between Cylinders". In: J. Heat Transfer 83 (1961), p. 415.

[92] H.K. Forster and N. Zuber. "Bubble Dynamics and Boiling Heat Transfer". In: AICHE J 1 (1955), pp. $532-535$.

[93] W.T. Hancox and W.B. Nicoll. "A General Technique for the Prediction of Void Distributions in Nonsteady Two-Phase Forced Convection". In: Int. J. Heat and Mass Transfer 14 (1971).

[94] Z. Karoutas and other. Subcooled Boiling Data From Rod Bundles. Tech. rep. 1003383. The Electric Power Research Institute (EPRI), Palo Alto, CA, 2002.

[95] D.C. Groenveld and K.K. Fung. Forced Convective Transition Boiling-Review of Literature and Comparison of Predictive Methods. Tech. rep. AECL-5543. Chalk River Nuclear Laboratories, 1976.

[96] G.E. McCreery et al. Thermal-Hydraulic Analysis of Semiscale MOD-1 Reflood Test Series (Gravity Feed Tests). Tech. rep. TREE - NUREG - 1010. Nuclear Regulatory Commission, 1977. 
[97] L.A. Bromley. "Heat Transfer in Stable Film Boiling". In: Chemical Engineering Progress 46.5 (1950), pp. 221-226.

[98] E.N. Ganic and W.M. Rohsenow. "Dispersed Flow Heat Transfer". In: Int. J. Heat and Mass Transfer 20 (1977), pp. 855-866.

[99] K.H. Sun, J.M. Gonzalez-Santalo, and C.L. Tien. "Calculations of Combined Radiation and Convection Heat Transfer in Rod Bundles Under Emergency Cooling Conditions". In: ASME Journal of Heat Transfer (1976).

[100] E.M. Sparrow and R.D. Cess. Radiation Heat Transfer. McGraw-Hill, 1977, p. 231.

[101] C.Y. Paik et al. Analysis of FLECHT-SEASET 163-Rod Blocked Bundle Data using COBRA-TF. Tech. rep. NUREG/CR-3046. United States Nuclear Regulatory Commission, 1985.

[102] A. Yamanouchi. "Effect of Core Spray Cooling in Transient State after Loss-of-Cooling Accident". In: J. Nucl. Science and Technology 5 (1968), pp. 547-558.

[103] J.H. McFadden et al. RETRAN-02 A Program for Transient Thermal-Hydraulic Analysis of Complex Fluid Flow Systems, Volume 1: Equations and Numbers. Tech. rep. NP-1850. Electric Power Research Institute, 1980.

[104] American Society of Mechanical Engineers. ASME Steam Tables. 1968.

[105] L. Haar, J.S. Gallagher, and G.S. Kell. NBS/NRC Steam Tables. Hemisphere Publishing Corporation, New York, 1984.

[106] J.H. Keenan and F.G. Keyes. Thermodynamic Properties of Steam. John Wiley \& Sons, New York, 1936.

[107] W. Wagner and A. Kruse. Properties of Water and Steam. The Industrial Standard IAPWS-IF97. Springer, 1998.

[108] IAPWS. Revised Release on the IAPWS Industrial Formulation 1997 for the Thermodynamic Properties of Water and Steam. Tech. rep. The International Association for the Properties of Water and Steam, Lucerne, Switzerland, 2007.

[109] IAPWS. Release on the IAPWS Formulation 2011 for the Thermal Conductivity of Ordinary Water Substance. Tech. rep. The International Association for the Properties of Water and Steam, Czech Republic, 2011.

[110] IAPWS. Release on the IAPWS Formulation 2008 for the Viscosity of Ordinary Water Substance. Tech. rep. The International Association for the Properties of Water and Steam , Berlin, Germany, 2008.

[111] IAPWS. IAPWS Release on Surface Tension of Ordinary Water Substance. Tech. rep. The International Association for the Properties of Water and Steam, 1994.

[112] D. F. Williams, L. M. Toth, and K. T. Clarno. Assessment of Candidate Molten Salt Coolants for the Advanced High-Temperature Reactor (AHTR). Tech. rep. ORNL/TM-2006/69. Oak Ridge National Laboratory, 2006.

[113] W.G. Luscher, K.J. Geelhood, and I.E. Porter. Material Property Correlations: Comparisons between FRAPCON-4.0, FRAPTRAN-2.0, and MATPRO. PNNL-19417 Rev. 2. Pacific Northwest National Laboratory. Sept. 2015.

[114] Held P.C. and D.R. Wilder. "High temperature hemispherical spectral emittance of uranium oxides at 0.65 and 0.70 mircometers". In: Journal of the American Ceramic Society 52.4 (Apr. 1969), pp. 182185 . 
[115] Cabannes MMF, JP Stora, and J Tsakiris. "Facteurs de reflexion et d'emission de UO2 a haute temperature". In: C. R. Acad. Sc. 264B (1967), pp. 45-48.

[116] T. T. Claudson. Emissivity Data of Uranium Dioxide. Report AW-55414. Nov. 1958.

[117] D.L. Hagrman, G.A. Reymann, and R.E. Mason. MATPRO - Version 11 (Revision 2): A Handbook of Materials Properties for Use in the Analysis of Light Water Reactor Fuel Rod Behavior. Tech. rep. NUREG/CR-0497, TREE-1280, Revision 2. U.S. Nuclear Regulatory Commission, 1981.

[118] P.E. MacDonald et al. MATPRO - Version 9: A Handbook of Materials Properties for Use in the Analysis of Light Water Reactor Fuel Rod Behavior. Tech. rep. TREE-NUREG-1005. Idaho National Engineering Laboratories, 1976.

[119] Thermal Conductivity Measurements of High Burnup UO2 Pellet and a Benchmark Calculation of Fuel Center Temperature. ANS International Topical Meeting on LWR Fuel Performance. Portland, Oregon, Mar. 1979.

[120] Lanning et al. FRAPCON-3 Updates, Including Mixed-Oxide Fuel Properties. Tech. rep. NUREG/CR6354, Vol.4, PNNL-11513. U.S. Nuclear Regulatory Commission, 2005.

[121] Geelhood K.J. Lusher W.G. Material Property Correlations: Comparisons between FRAPCON-3.4, FRAPTRAN 1.4, and MATPRO. Technical Report NUREG/CR-7024. Richland, Washington: Pacific Northwest Laboratory, 2011.

[122] Lanning et al. FRAPCON-3: Modifications to Fuel Rod Material Properties and Performance Models for High-Burnup Application. Technical Report NUREG/CR-6534, Vol.1. U.S. Nuclear Regulatory Commission, 1997.

[123] M. Amaya et al. "Thermal conductivity evaluation of high burnup mixed- oxide (MOX) fuel pellet". In: Journal of Nuclear Materials 404 (2011), pp. 303-308.

[124] Thermophysical properties database of materials for light water reactors and heavy water reactors. Final report of a coordinated research project: 1999-2005 IAEA-TECDOC-1496. IAEA, June 2006.

[125] Brooks CR and EE Stansbury. "The specific heat of Zircaloy-2 from 50 to $700^{\circ} \mathrm{C}$ ". In: Journal of Nuclear Materials 18 (1966), p. 223.

[126] Deem HW and EA Eldridge. Specific Heats of Transformation of Zirclaoy-2 and Low Nickel Zircaloy-2. Tech. rep. USAEC BM1-1803. Columbus, Ohio: Battelle Memorial Institute, 1967.

[127] Coughlin JP and EG King. "High-temperature heat contents of some zirconium-containing aubstances". In: Journal for the American Chemical Society 72 (1950), p. 2262.

[128] Peggs ID and DP Godin. "The yield strength-hot hardness relationship of zircaloy-4." In: Journal of Nuclear Materials 57 (1975), pp. 246-248.

[129] D.B. Scott. Physical and Mechanical Properties of Zircaloy-2 and -4. Tech. rep. WCAP-3269-41. Idaho National Engineering Laboratories, 1965. 